Florida International University FIU Digital Commons

$7-1-2016$

\title{
Development of a Primary Ion Column for Mass Spectrometry-Based Surface Analysis
}

Raul A. Villacob

rvillo28@fiu.edu

DOI: $10.25148 /$ etd.FIDC000761

Follow this and additional works at: https://digitalcommons.fiu.edu/etd

Part of the Analytical Chemistry Commons

\section{Recommended Citation}

Villacob, Raul A., "Development of a Primary Ion Column for Mass Spectrometry-Based Surface Analysis" (2016). FIU Electronic Theses and Dissertations. 2561.

https://digitalcommons.fiu.edu/etd/2561 


\section{FLORIDA INTERNATIONAL UNIVERSITY}

Miami, Florida

DEVELOPMENT OF A PRIMARY ION COLUMN FOR MASS SPECTROMETRYBASED SURFACE ANALYSIS

A thesis submitted in partial fulfillment of the requirements for the degree of

MASTER OF SCIENCE

in

CHEMISTRY

by

Raul A. Villacob

2016 
To: Dean Michael R. Heithaus

College of Arts, Sciences and Education

This thesis, written by Raul A. Villacob, and entitled Development of a Primary Ion Column for Mass Spectrometry-based Surface Analysis, having been approved in respect to style and intellectual content, is referred to you for judgment.

We have read this thesis and recommend that it be approved.

Piero R. Gardinali

Jeffrey Joens

Francisco A. Fernandez-Lima, Major Professor

Date of Defense: July 12016

The thesis of Raul A. Villacob is approved.

Dean Michael R. Heithaus

College of Arts, Sciences and Education

Andrés G. Gil

Vice President for Research and Economic Development and Dean of the University Graduate School

Florida International University, 2016 


\section{DEDICATION}

This work is dedicated to my family, as with your nurturing and support I would not have been able to accomplish this work. It is also dedicated to my friends who have been there to help me throughout the situational rollercoaster the past 2 years have been. 


\section{ACKNOWLEDGMENTS}

I would like to start by thanking my advisor, Dr. Francisco Fernandez-Lima. You decided to give me an opportunity 3 years ago that has changed the course of my life. Joining your lab has given me the opportunity of finding my place as both a chemist and an engineer in terms of scientific acumen and creativity. Even though we bump heads at times, I am very much looking forward to continuing my work under your guidance for my Ph.D studies. I would also like to thank my former mentor Dr. Daniel DeBord. His enthusiasm and patience set me on track to become proficient in terms of understanding how instrumentation development works. I would also like to thank my committee members Dr. Jeffrey Joens and Dr. Piero Gardinali, as your input throughout the writing of this thesis has been very valuable.

I would like to thank the members of the group for their support in terms of bouncing ideas, listening to my rants and being encouraging all around. Having such a enjoyable work environment with other goal-oriented individuals has made this experience a lot less grueling. In particular I would like to thank Paolo Benigni and Anthony Castellanos, as your support in my initial experiments meant a lot to me, whether it was getting bummed out when things would not work or being there when I was performing experiments in the lab. I would like to thank my dad for his unwavering support throughout the time I have been working on this thesis. Your love and patience to listen and discuss my work with me has also been huge when I've found myself stuck in difficult steps. I would also like to thank my mother for nurturing a persistent attitude that helped me overcome the hurdles I have stumbled upon and her patience to raise such a hard-headed person. 


\title{
ABSTRACT OF THE THESIS \\ DEVELOPMENT OF A PRIMARY ION COLUMN FOR MASS SPECTROMETRY- BASED SURFACE ANALYSIS
}

by

\author{
Raul A. Villacob
}

Florida International University, 2016

Miami, Florida

\begin{abstract}
Professor Francisco A. Fernandez-Lima, Major Professor
Secondary Ion Mass Spectrometry (SIMS) is a powerful technique for high spatial resolution chemical mapping and characterization of native surfaces. The use of massive cluster projectiles has been shown to extend the applicable mass range of SIMS and improve secondary ion yields 100 fold or beyond. These large projectiles however, present a challenge in terms of focusing due to the initial spatial and kinetic energy spreads inherent to their generation. In the present work, we describe the development and construction of a novel primary ion (PI) column employing a gold nanoparticle liquid metal ion source (AuNP-LMIS) and the coupling to ultrahigh resolution mass spectrometers (e.g., Fourier Transform Ion Cyclotron Resonance Mass Spectrometer, FTICR MS) for accurate chemical characterization of complex biological surfaces. This work describes the ion dynamics, development and the experimental characterization of the AuNP-LMIS.
\end{abstract}




\section{TABLE OF CONTENTS}

CHAPTER

PAGE

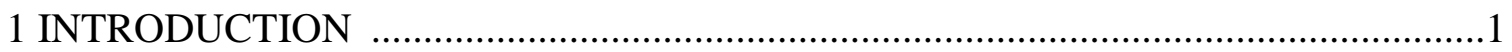

1.1 Surface Analysis and Mass Spectrometry Imaging ........................................1

1.2 Secondary Ion Mass Spectrometry and Operational Regimes...............................4

1.3 Motivation for the Research of Primary Ion Sources in SIMS: Mechanistic and

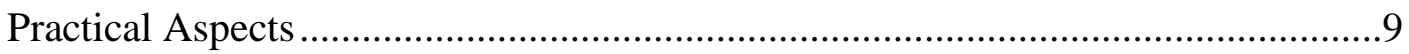

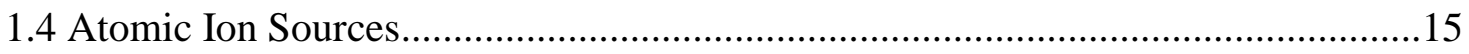

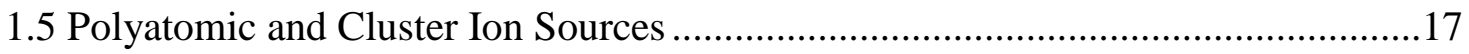

1.6 Massive Clusters and Charged Nanodroplets ..................................................26

2 DEVELOPMENT OF A MULTI-SOURCE PRIMARY ION COLUMN......................35

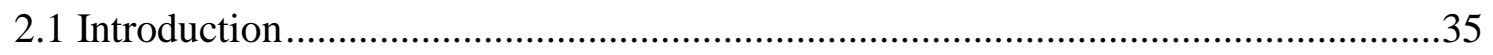

2.1.1 Ion Optics and Electrostatic Lens .........................................................

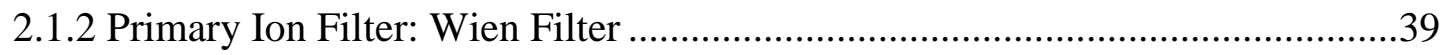

2.1.3 Collimation and Electrostatic Deflectors: Pulsing ......................................41

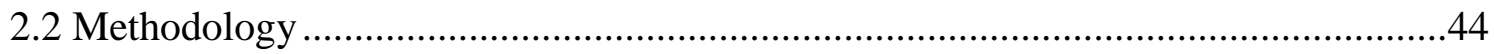

2.2.1 Design (Solidworks/CAD) and Fabrication................................................44

2.2.2 SIMION Simulations for Value Optimization ............................................45

2.3 Results and Discussion .............................................................................4

2.3.1 Solidworks (CAD) Designs of Primary Ion Column Components.....................47

2.3.2 SIMION Array of Primary Ion Column Components .....................................54

2.3.3 Finalized Assembly of the Primary Ion Column and Further Modifications ......59

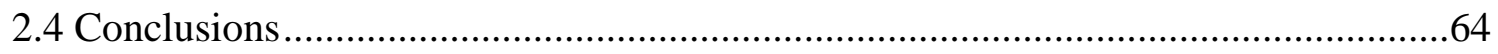


3 EVALUATION OF THE AU-LMIS AND INCORPORATION TO THE COLUMN .65

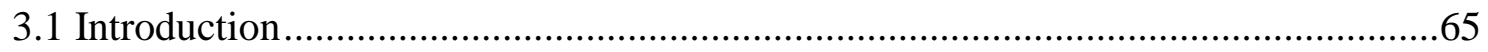

3.2 Methodology for Source Incorporation and Testing ..........................................71

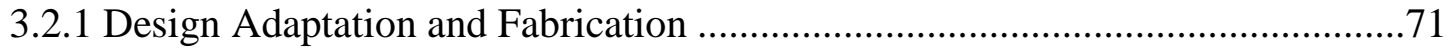

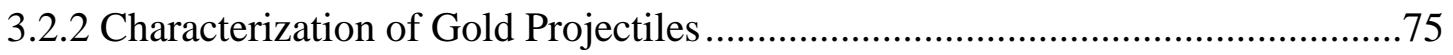

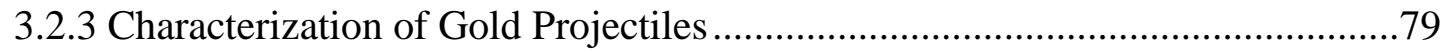

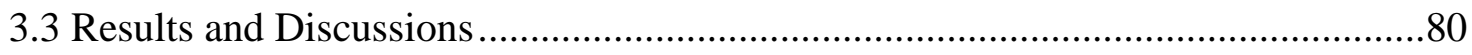

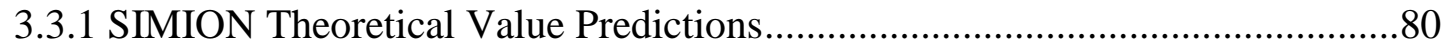

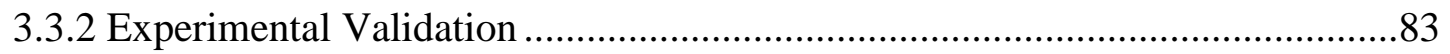

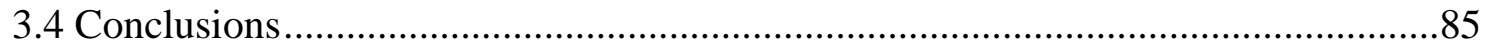

4 INTEGRATION OF THE PRIMARY ION COLUMN WITH AN FT-ICR MS .........87

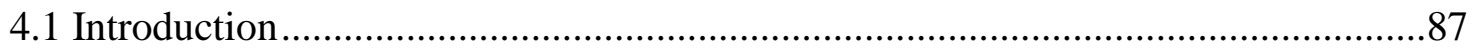

4.2 Methodology for Interface Development and Testing .....................................93

4.2.1. Design of the Interfacing Sector and Extraction Optics ................................94

4.2.2 Development of a Total Simulation Involving Primary Ion Column, Interfacing

Optics and Secondary Ion Extraction Optics ................................................98

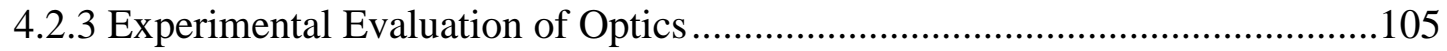

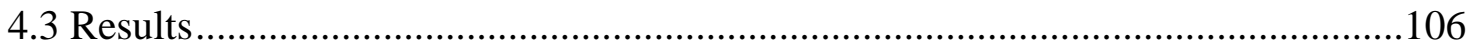

4.3.1 Theoretical Optimization of Primary Ion Column with Interfacing Optics

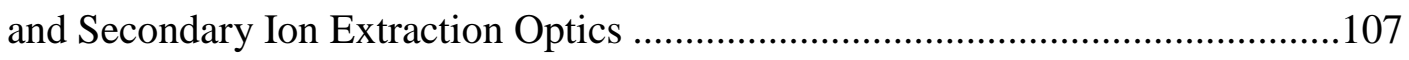

4.3.2 Construction of the Interfacing Optics and Final Instrument Assembly .........115

4.3.3 Experimental Evaluation of Secondary Ion Extraction Lens.........................118

4.3.3.1 Theoretical Optimization of Primary Ion Column with Interfacing Optics and Secondary Ion Extraction Optics 
4 3.3.2 Development of the Vacuum Electrospray Source for Standalone Ion Optics

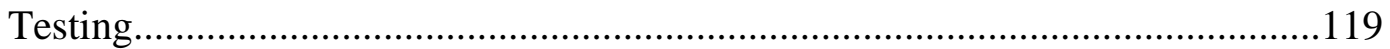

4.3.3.3 Assembly of the Test Bench and SI Lens Profiling ...................................121

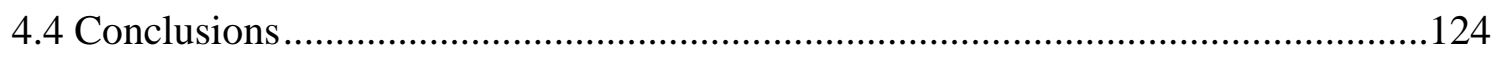

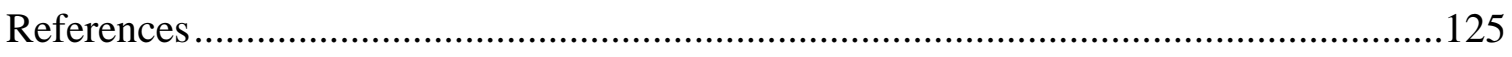

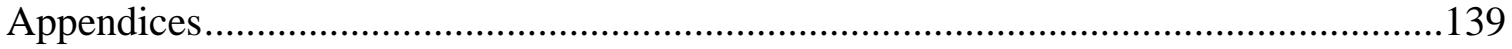




\section{LIST OF FIGURES}

\section{FIGURE}

PAGE

Figure 1. Mass range and spatial resolution of common MSI techniques.

Figure 2. Depiction of SIMS process. A primary ion impacts the upper sample monolayers, generating photons, electrons, secondary ions and neutrals... .5

Figure 3. Comparison of sputtering mechanisms based on projectile size. Smaller projectiles engage in a binary collision cascade, while larger projectiles generate localized surface plasma's leading to thermal spiking.

Figure 4. IONTOF 20 kV C60 Primary Ion Column. Reproduced with permission of IONTOF GmbH (Munster, Germany).

Figure 5. Comparison of negative ion mode spectra for high-energy $\mathrm{Au}_{3}{ }^{+}, \mathrm{Au}_{9}{ }^{+}$, and $\mathrm{Au}_{400}{ }^{+4}$ projectiles on a native rat brain section. Note the significant increase in overall SI yield (intensity) and greater molecular ion intensity and variety, and generation of intact molecular lipids ( $~ 900 \mathrm{~m} / \mathrm{z}$ region) when employing $\mathrm{Au}_{400}{ }^{+4}$. Reprinted with permission from FFL et al (2012). Copyright 2012 American Chemical Society.

Figure 6. Schematic of an IONTOF Argon Gas Cluster Primary Ion Column (ArGCIB). Reproduced with permission of IONTOF GmbH (Munster, Germany)......

Figure 7. Timeline of the crucial developments in Secondary Ion Mass Spectrometry with an emphasis on the advancement of projectiles and operation modes.

Figure 8. Scheme for a simple, symmetrical 3 electrode aperture lens. Relations between features dimensions demonstrated as a function of the aperture size, D.

Figure 9. Scheme for a deacceleration-acceleration aperture einzel lens $(\mathrm{V} 1=\mathrm{V} 3=0)$. Note the varying aperture sizes and their respective ratios, as these influence focal length and focal strength (D/f).

Figure 10. 3D cutaway view of the design for the pre-filter einzel lens with potential values and key structural features highlighted.

Figure 11.3D cutaway view of the design for the Wien Filter with key components highlighted.

Figure 12. 3D cutaway view of the design for the primary ion column with key components for beam modification highlighted. 
Figure 13. 3D view of the first set of electrostatic deflection plates for beam pulsing and alignment with key components highlighted.

Figure 14. 3D cutaway view of the XY-controllable beam collimation array with key components highlighted.

Figure 15. 3D view of the post-filter einzel lens and secondary set of electrostatic deflection plates with key components highlighted.

Figure 16. Simulation view of the pre filter-einzel lens performance with optic geometry features highlighted...

Figure 17. 3D view of Wien Filter performance simulation for selection of massive gold clusters with components highlighted....

Figure 18. 3D view of post-filter einzel lens and electrostatic plates

(deflection/pulsing) simulation performance with components highlighted.

Figure 19. 2D XY and 3D simulation of the potential energy lines and surfaces for primary ion column components.

Figure 20. SIMION analysis for comparing beam cross-sections based on Wien Filter electrodes' geometry.

Figure 21. Side view of initial and final primary ion column design with key differences and features highlighted.

Figure $22^{73}$. Schematic of primary ion column for the characterization of $\mathrm{Au}$ cluster projectiles utilizing time of flight measurements. Reprinted with permission from M. Benguerba. Copyright 1991 Elsevier B.V.

Figure $23^{73}$. Results from the Au projectile TOF characterization experiments highlighting different cluster sizes $(n=1-7)$. Larger projectiles have greater times of flight. Reprinted with permission from M. Benguerba. Copyright 1991 Elsevier B.V.

Figure $24^{73}$. Comparison of secondary ion yields between gold projectiles $n=1-5$, $\mathrm{q}=1-2$ as a function of projectile kinetic energy. Reprinted with permission from M. Benguerba. Copyright 1991 Elsevier B.V.

Figure $25^{99}$. Measured distributions of cluster sizes (n) as a function of the amount of charges (q). Note that the heavier the cluster the more charges are available. Reprinted with permission from Della-Negra. Copyright 2004 Elsevier B.V. 69

Figure $26^{101}$. Schematic for the ion and electron optics on the PEGASE instrument demonstrating sequence of signaling for single-impact detection. 
Reprinted with permission from FFL et al (2012). Copyright 2012 American

Chemical Society.

Figure 27. 3D cross-section view of Au-LMIS structure, with the insert highlighting a top view perspective of a prepared emitter utilized in characterization experiments.

Figure 28. Specialized electronics for powering the $20 \mathrm{kV}$ LMIS inside the primary ion column with components and connections highlighted. 73

Figure 29. Electronics wiring diagram for the LMIS. 73

Figure 30. Comparison of early and current LMIS structure, mounting systems, and feedthrough. 75

Figure 31. Primary ion generation and trajectory conditions for simulation.

Figure 32. XY simulation view of the Au-LMIS and pre-filter einzel lens illustrating the trajectory of the primary ions upon generation and subsequent emission.

Figure 33. 3D view for ion optics simulation workbench for the theoretical evaluation of optics performance with components labeled.

Figure 34. Au400+4 Beam cross-section profiles for low current-high focus (microprobe) and a high current-low focus (sputter) operational modes at a focal distance of $1173.6 \mathrm{~mm}$, the exit of the primary ion column.

Figure 35. Simulations with optimized potential values for obtaining A) a high current sputter beam for dynamic SIMS applications and B) A low current, highly focused beam for nanoscale static SIMS.

Figure 36. Results from Au-LMIS projectile characterization employing the Wien Filter with an emphasis of detection of massive gold clusters $\left(\mathrm{Au}_{400}{ }^{+4}\right)$.

Figure 37. Camera view of bombarded phosphorous screen for beam diameter profiling. Focusing for the experiment above produced an $\mathrm{Au}_{400}{ }^{+4}$ beam with a diameter of $\sim 1 \mathrm{~mm}$

Figure 38. Reproduced with permission from DeBord, FFL, et $\mathrm{al}^{91}$. A),B), and C) show sub-micron spatial resolution imaging capabilities of the TOFSIMS5, yet the lack of resolving power makes chemical identification challenging at the selected $\mathrm{m} / \mathrm{z}(\mathrm{m} / \mathrm{z} 277)$. D) and $\mathrm{E})$ show the significant mass resolving power of the $\mathrm{C}_{60}{ }^{+}$FT-ICR MS instrument, with 5 characteristic peaks assigned within the $277 \mathrm{~m} / \mathrm{z}$ mass window. 
Figure 39. 3D view of design for electrostatic sector to deflect the PI beam towards the FT-ICR MS.

Figure 40. 3D view of primary ion beam rastering deflector plates with mounts for lens addition.

Figure 41. 3D view of secondary ion extraction lens system. Additional functionality features are highlighted.

Figure 42. 3D isometric view of the simulation for evaluating secondary ion extraction lens performance and subsequent transmission into the SolariX's first component (Q1).

Figure 43. SIMION isometric view of total simulation with components labeled..... 100

Figure 44. Design for standalone secondary ion extraction lens test bench with components highlighted and labeled. Insert show the specialized electronics utilized to generate a stable ionic liquid beam and the assembled secondary ion extraction lens.

Figure 45. Results from the profiling of primary ion beam focus and transmission after velocity-selection and collimation.

Figure 46. Results from the Negative Ion Mode Beam Profiling.

Figure 47. Results of the Positive Ion Mode Beam Profiling.

Figure 48. Simulation of optimized voltage performance for transmitting ions of varying mass into the first quadrupole of the SolariX, followed by the collisional cell (Q2). Ions of mass ranges 150-300 m/z, 250-750 m/z, and 400-1100 m/z. Three packets of 10,000 ions were flown simultaneously accounting for coulombic repulsion.

Figure 49. Evaluation of secondary ion transmission based on $\mathrm{m} / \mathrm{z}$ utilizing simulation derived values. Black data points accounting for energy, $\mathrm{m} / \mathrm{z}$, and Xdisplacement (transmission), while red data points account for displacement account for mass and displacement alone.

Figure 50. 3D view of the completed simulation with all optimized voltages highlighted. Simulation displays the trajectory of the focused primary ion beam and the transmission of the generated secondary ions into the FT-ICR MS Q1

Figure 51. Side view of the completed AuNP-FT-ICR MS assembly with main components highlighted.

Figure 52. Design, fabrication and assembly of the vacuum electrospray source. A) Design for prototype source consists of a modified CF 2.75 in flange B). 
Photograph of assembled prototype flange. Total internal length is $\sim 2.2$ in. C)

Photograph of a freshly etched polyamide/fused silica nanoelectrospray capillary.... 120

Figure 53. Electronics wiring diagram for alternating polarity HV power supply...... 120

Figure 54. View of the assembled test bench with all relevant components labeled (with the exception of the 255HP Turbomolecular Pump). S.I. lens not pictured as these are located within the testing chamber.

Figure 55. Solidworks cutaway view of test bench configuration for the evaluation of SI extraction lens transmission $(\mathrm{A} / \mathrm{sec})$

Figure 56. Experimental Ion Transmission Evaluation of the Secondary Ion Extraction Lens as a function of extractor block voltage. Note that max transmission is achieved with the theorized simulation value. 


\section{ABBREVIATIONS AND ACRONYMS}

SIMS Secondary Ion Mass Spectrometry

MALDI Matrix-Assisted Laser Desorption Ionization

LESA Liquid Extraction Surface Analysis

DESI Desorption Electrospray Ionization

LAESI Laser-Assisted Electrospray Ionization

FT-ICR Fourier Transform-Ion Cyclotron Resonance

FT-MS Fourier Transform-Mass Spectrometry

MS Mass Spectrometry

TOF Time of Flight

DC Direct Current

$\mathrm{AC} \quad$ Alternating Current

RF Radio Frequency

PI Primary Ion

SI Secondary Ion

AuNP Massive Gold Nanoparticle

MSI Mass Spectrometry Imaging

LOD Limits of Detection

q-TOF Quadrupole-Time of Flight

BSA Binary Collision Approximation

LMIS Liquid Metal Ion Source

ICP Inductively Coupled Plasma

MD Molecular Dynamics

IPNO Institute of Nuclear Physics Orsay 


\begin{tabular}{|c|c|}
\hline MCI & Massive Cluster Impact \\
\hline MCP & Multichannel Collector Plate \\
\hline ILS & Ionic Liquid Source \\
\hline EDI & Electrospray Droplet Impact \\
\hline GCIB & Gas Cluster Ion Beam \\
\hline $\mathrm{CNC}$ & Computer Numeric Control \\
\hline CAD & Computer-Assisted Design \\
\hline STL & Stereolitography \\
\hline PEEK & Polyether Ether Ketone \\
\hline CMOS & Complimentary Metal-Oxide Semiconductor \\
\hline PSD & Position Sensitive Detector \\
\hline AFM & Atomic Force Microscopy \\
\hline TEM & Transmission Electron Microscopy \\
\hline TDC & Time to Digital Converter \\
\hline FWHM & Full Width at Half Max Height \\
\hline ESI & Electrospray Ionization \\
\hline MMA & Measured Mass Accuracy \\
\hline OLED & Organic Light-Emitting Diode \\
\hline LED & Light-Emitting Diode \\
\hline PNNL & Pacific Northwestern National Laboratory \\
\hline MHV & Mini-High Voltage Connector \\
\hline $\mathrm{BNC}$ & Bayonet-Neill-Concelman Connector \\
\hline SHV & Strong High Voltage Connector \\
\hline
\end{tabular}




\section{INTRODUCTION}

\subsection{Surface Analysis and Mass Spectrometry Imaging.}

An ongoing pursuit in the analytical community has been the characterization and chemical mapping of micro and nanoscale objects ${ }^{1}$ in a way to better understand the chemical makeup, both organic and inorganic, of the main constituents of biological systems ${ }^{2}$. In the past 20 years, Mass Spectrometry Imaging (MSI) instrumentation has become the technique of choice for characterization and chemical mapping of substances in complex matrices at micrometric and submicrometric scales ${ }^{3}$. Mass spectrometrybased surface characterization is not defined by one particular approach, but rather encompasses a variety of probes and mass analyzers. For example, MSI techniques can be categorized by the nature of the probe, their varying ionization mechanisms and instrumental conditions (e.g. vacuum vs ambient) ${ }^{4}$. These features define the effective mass range and spatial resolution characteristic to each probe, thereby also defining the applications $^{2,3,5}$. Matrix-Assisted Laser Desorption (MALDI), Laser Electrospray Ambient Ionization (LAESI), Liquid Extraction Surface Analysis (LESA), and Desorption Electrospray Ionization (DESI) are ambient techniques that have shown success in chemical imaging of cell cultures and fresh tissue samples ${ }^{5,6}$. In addition, ambient techniques can be easily integrated with post-ionization separation techniques

like ion mobility spectrometry (IMS) and tandem MS/MS systems ${ }^{3,7}$. Traditionally, other ion beam based vacuum probes based have provided better spatial resolution (Secondary Ion Mass Spectrometry, SIMS $)^{8}$. While progress have been made in both ambient and vacuum technologies, the ability to interrogate submicrometric domains remains 
challenging (see Figure 1 for typical application range) ${ }^{3,9}$. In addition to the spatial resolution, recent advances have permitted the 3D-chemical mapping using SIMS.

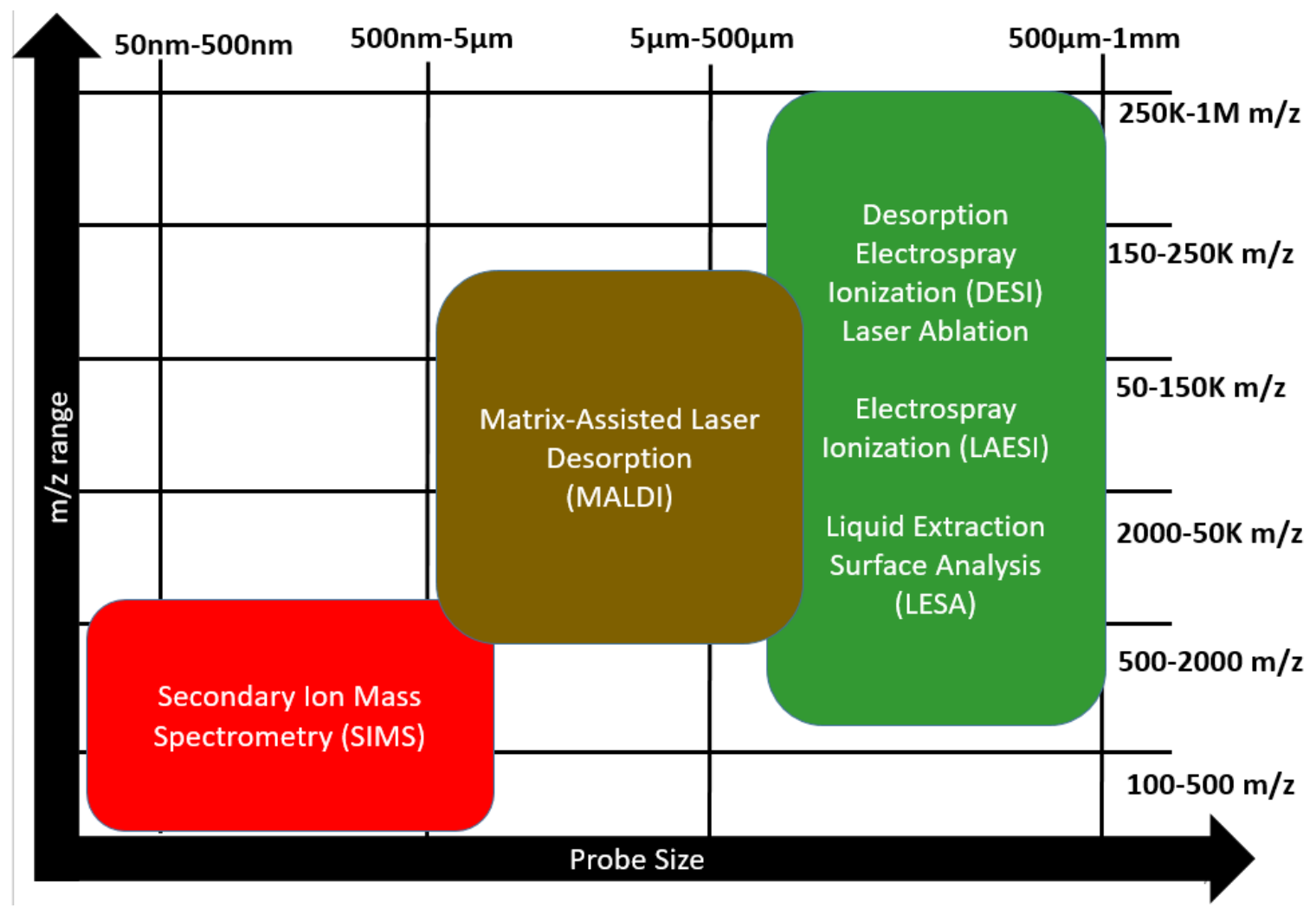

Figure 1. Mass range and spatial resolution of common MSI techniques.

A brief description of the different MSI techniques follows:

Secondary Ion Mass Spectrometry (SIMS): Secondary Ion Mass Spectrometry is a vacuum technique that utilizes a energetic primary ion (PI) beam (keV-MeV) to desorb/sputter material from a surface ${ }^{10}$. Some of this material may become ionized, becoming secondary ions (SI) during the desorbtion process by different mechanisms, dependent on the projectile size and energy and sample nature (organic or inorganic). SIMS provides high spatial resolution for both 2D and 3D chemical imaging $(<300$ $\mathrm{nm})^{10}$. Resolution is primarily limited by the PI beam focus at the target, which can be 
controlled employing ion optics and time-pulsing schemes (IO). The SIs are mainly composed of singly charged fragments and intact molecular ions of the surface of interest $^{11}$.

Matrix-Assisted Laser Desorption Ionization (MALDI). Matrix Assisted Laser Desorption Ionization is a pressure-independent surface technique. The sample surface of interest is coated with a protic matrix, which is exposed to laser pulses (e.g., 337 and $355 \mathrm{~nm})^{12}$. This process occurs by a coherent phonon excitation via pulsed laser shots that leads the matrix and sample to form a localized hot plume of gas, interacting with the sample surface $^{12}$. At this stage, these interactions can foster the generation of ions via gentle protonation and deprotonation processes. This technique is one of the most popular MSI technique, having widespread usage in the analysis of biological and organic materials thanks to the ability of generating molecular ions ranging of up to $100,000 \mathrm{~m} / \mathrm{z}$, with a typical spatial resolution in the tens of microns, and ease of hyphenation with other techniques $^{3,9}$. The matrix crystal size often defines the spatial resolution limit and the mass range of the analysis ${ }^{3,9}$.

Desorption Electrospray Ionization (DESI). Desorption Electrospray Ionization is an ambient technique where ions are generated by directing a focused jet stream of electrosprayed droplets to a sample surface ${ }^{7}$. Droplets from this stream impact the sample, expand, and desorb material as they recoil back into the gas phase generating mostly intact molecular ions ${ }^{3}$. Thanks to the gentleness of the ionization mechanism, minimal damage is done to the morphology of the sample and it can complemented with other imaging techniques for $2 \mathrm{D}$ and $3 \mathrm{D}$ analysis ${ }^{5}$. 
Laser Ablation Electrospray Ionization (LAESI). Laser Ablation Electrospray Ionization combines DESI and MALDI to provide high spatial resolution MSI, along with 2D and 3D chemical mapping capabilities ${ }^{13}$. A mid-infrared (IR) laser ablates material from the sample surface and an orthogonal electrospray source emitting charged droplets provides further ionization of the ejected material via charge exchange mechanisms similar to conventional electrospray ionization (ESI) ${ }^{13}$. The spatial resolution is typically limited by the laser spot size.

Liquid Extraction Surface Analysis (LESA). Liquid Extraction Surface Analysis is an ambient ionization technique, where a nanoelectrospray pipette is directed to a site on the sample to perform a localized solid-liquid extraction ${ }^{14}$. The probing tip is then transferred to a mass spectrometer inlet, where the extracted material is ionized via electrospray ionization. LESA can be utilized for 2D and 3D imaging, including single-cell analysis for certain eukaryotic species by utilizing ultra-fine nanocapillaries for sample probing ${ }^{14}$. It must be noted that there are other variants of MSI but SIMS stands alone as the main technique for nanoscale 2D and 3D imaging at the intra-cellular level ${ }^{11,15}$.

\subsection{Secondary Ion Mass Spectrometry and Operational Regimes}

Over the last decades, SIMS has become the go-to tool for the characterization

of native untreated surfaces at the microscale and nanoscale ${ }^{10-11}$. The sensitivity for SIMS measurements extremely high when compared to other MSI methods due to the nature of the interrogation, allowing for routine detection of atomic species at parts per billion, with a dynamic range of $10^{9}$ when coupled with multiple detectors or multichannel 
detectors ${ }^{10}$. Fluctuations in the SI emission are fairly common and quantification typically requires the usage of reference materials ${ }^{10}$.

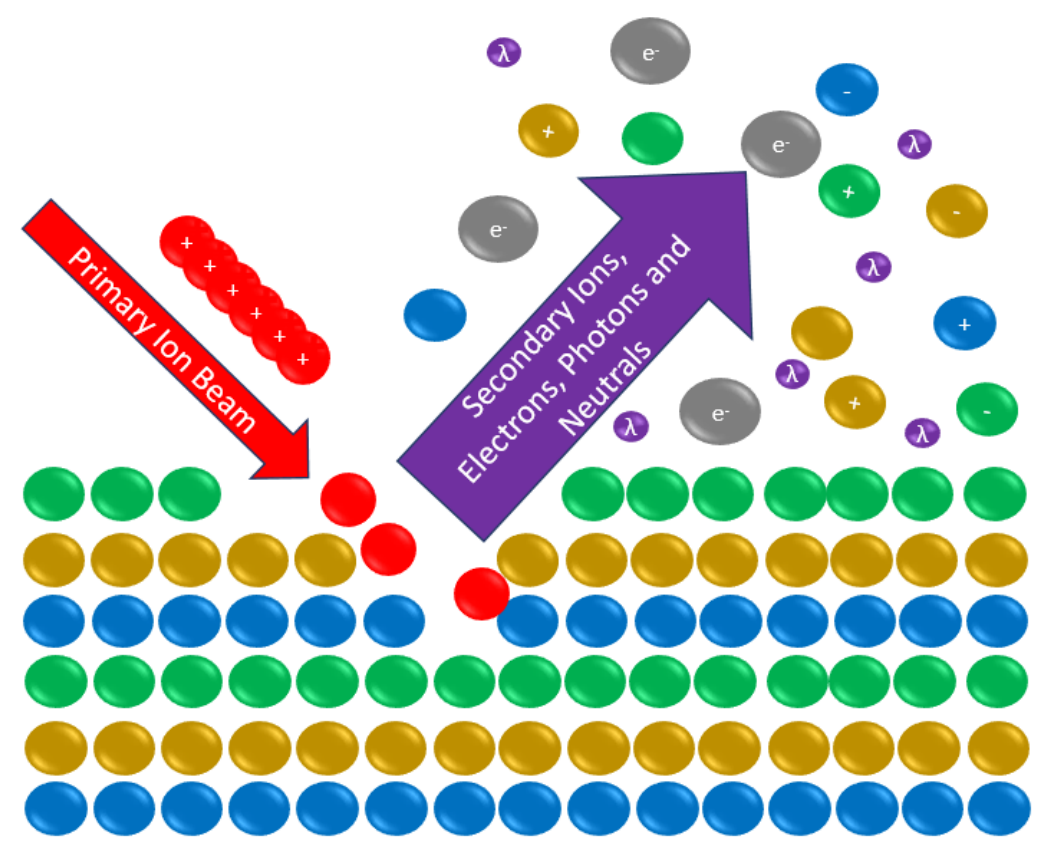

Figure 2. Depiction of SIMS process. A primary ion impacts the upper sample monolayers, generating photons, electrons, secondary ions and neutrals.

SIMS operational modes depend on the analysis of uppermost monolayers of the sample or the analysis of the composition as a function of depth, referred to as Static SIMS and Dynamic SIMS, respectively ${ }^{10}$. These modes differ in the PI dose or number of impacts per $\mathrm{cm}^{2}$. In static SIMS, this goal is achieved by bombarding the sample surface with less than $10^{13}$ ions $/ \mathrm{cm}^{2}$ for the given field of view. Beam fluence, or dose density beneath this threshold is referred to as the staying within the static limit. Analyses conducted under the static limit perturb less than $1 \%$ of the irradiated surface of the sample, allowing the overall morphology relatively intact ${ }^{2,10}$. Within the static limit, damage is generally reduced to the upper sample monolayers, allowing the subsurface of the sample to go mostly undisturbed ${ }^{16}$. However, the low sputter yields associated with 
common projectiles coupled with the poor ionization efficiency yields low number of SIs ${ }^{17}$. In surpassing the static limit $\left(>1 \times 10^{13}\right.$ ions $\left./ \mathrm{cm}^{2}\right)$ one enters the range of Dynamic SIMS. In Dynamic SIMS a continuous beam of ions (DC or near DC) is employed where the number of incident ions exceeds the atom count at the surface, leading to a gradual sample erosion ${ }^{10}$. Ion dosages in this mode generally are in excess of $1.0 \times 10^{14}$ ions $/ \mathrm{cm}^{2}$, thus damage to the sample is far more pronounced, due to irreversible changes in the morphology of the sample ${ }^{9-10}$. Conventional atomic projectiles has been known to lead to loss of all molecular information, generally relegating the technique to elemental and small molecules ${ }^{18}$. Yet in this operational mode, one can thoroughly profile the chemical make-up of the sample as a function of depth. Dynamic SIMS also provides enhanced sputter and ionization yields, maximizing sensitivity for trace analysis ${ }^{10}$. However, advances in projectiles have allowed for Dynamic SIMS to become widely applicable by incurring significantly reduced damage ${ }^{19,20}$. Other advances capitalize on the DC-nature of the beam in this mode, allowing for nanoscale analyses to be performed with ease $(>250 \mathrm{~nm})^{15}$.

The 2D static SIMS operation is preferred in order to better localize analytes on the sample surface while minimizing sample damage ${ }^{10}$. When MSI is used, chemical images of the surface are generated by rastering the PI beam and co-locating the emitted SIs to that probing site ${ }^{10}$. A mass spectrum is acquired from each probed site and rendered as a pixel, with the collection of many points (pixels) for the 2D-SIMS image. In the case of 3D SIMS imaging, 2D data points are recorded with respect to their depth chemical information (XY vs Z), referred to as voxels, instead of pixels ${ }^{21}$. Dynamics SIMS is common for 3D-SIMS as the higher dosage allows for the necessary yields over 
time to generate voxels, consequently providing better limits of detection (LOD) over Static SIMS (2D imaging) $)^{22}$ A voxel considers both lateral (XY) and depth (Z) resolution in order to define the effective resolution ${ }^{21}$. Dynamic SIMS for 3D imaging has been characterized to have excellent depth resolution with damage reduced to the first 5-10 $\mathrm{nm}$ of the sample, depending on the PI employed ${ }^{23}$. In addition, the DC nature of the beam has allowed for the best recorded focusing possible in SIMS, with commercial instrumentation offering spatial resolutions of 50-200 $\mathrm{nm}$ to be achieved by experienced users $^{15}$

Most conventional molecular application-oriented SIMS instrumentation utilize time of flight (TOF) analyzers. These analyzers have very fast cycle times, as generation of SIMS images requires the collection of spectra from an average of $\sim 1$ million pixels ${ }^{10}$. Time of flight analyzers equipped with position-sensitive detectors are routinely capable of generating images in minutes, yet these lack the resolving power required to perform exact chemical identification of molecular ions from complex organic substrates, such as biological surfaces ${ }^{11}$. Table 1 shows typical probes and mass analyzers utilized to obtain different types of chemical data, from elemental to intact molecules. More about instrumental advancements in SIMS for mass analyzers will be discussed in Chapter 4. 


\begin{tabular}{|c|c|c|c|}
\hline Relevant Data & $\begin{array}{l}\text { Source/Projectile } \\
\text { Type }\end{array}$ & Mass Analyzer & Detector \\
\hline $\begin{array}{l}\text { Elemental/Small } \\
\text { Molecule Analysis }\end{array}$ & $\begin{array}{l}\text { Atomic Primary } \\
\text { Ion Beam }\end{array}$ & $\begin{array}{l}\text { - } \quad \text { Quadrupole (q) } \\
\text { - } \quad \text { Double Focusing Electrostatic- } \\
\text { Magnetic Sector (Mattuch-Herzog) }\end{array}$ & - Faraday Cup Array \\
\hline $\begin{array}{l}\text { Molecular Species } \\
\text { Analysis }\end{array}$ & $\begin{array}{l}\text { Polyatomic \& } \\
\text { Cluster Primary } \\
\text { lon Beam }\end{array}$ & $\begin{array}{ll}\text { - } & \text { Time of Flight (ToF) } \\
\text { - } & \text { Time of Flight-Time of Flight (ToF-ToF) } \\
\text { - } & \text { Fourier Transform lon Cyclotron } \\
& \text { Resonance (FT-ICR-MS) } \\
\text { - } & \text { Quadrupole-Time of Flight (Q-ToF) } \\
\text { - } & \text { Triple Quad (Q/Q/Q) }\end{array}$ & $\begin{array}{l}\text { - Multi-Channel Detector } \\
\text { Plate } \\
\text { - Position-Sensitive } \\
\text { - Detector } \\
\text { - Faraday Cup } \\
\text { - Ion Cyclotron Resonance } \\
\text { Cell (FT-ICR only) }\end{array}$ \\
\hline $\begin{array}{l}\text { Intact Molecular } \\
\text { Species Analysis } \\
\text { (I.e. Biomolecules, } \\
\text { Large Polymers) }\end{array}$ & $\begin{array}{l}\text { Massive Cluster } \\
\text { Primary Ion Beam }\end{array}$ & $\begin{array}{ll}\text { - } & \text { Time of Flight (ToF) } \\
\text { - } & \text { Time of Flight-Time of Flight (ToF-ToF) } \\
\text { - } & \text { Fourier Transform Ion Cyclotron } \\
& \text { Resonance (FT-ICR-MS) } \\
\text { - } & \text { Quadrupole-Time of Flight (Q-ToF) }\end{array}$ & $\begin{array}{l}\text { - Multi-Channel Detector } \\
\text { Plate } \\
\text { - Position-Sensitive } \\
\text { - Detector } \\
\text { - Faraday Cup } \\
\text { Ion Cyclotron Resonance } \\
\text { Cell (FT-ICR only) }\end{array}$ \\
\hline
\end{tabular}

Table 1. Description of current SIMS instrumentation. 
1.3 Motivation for the Research of Primary Ion Sources in SIMS: Mechanistic and Practical Aspects

The removal of material from a surface following the impact of a PI beam and the yields and efficiencies of these processes are rooted in the characteristic of the ionization mechanism. Ionization mechanisms vary as a function of the projectile size, composition and energy. Atomic PI sources are the standard probes for most SIMS applications, but they falter in the generation of significant molecular ion signals, especially intact secondary molecular ions. Such problem is rooted in that atomic projectiles generate fragments, as opposed to molecular ions due to their high impact energy. This section will focus on briefly highlighting the different ion-solid and ionization mechanisms (atomic and molecular), and their dependence with the type of projectiles.

Ion-solid interaction, sputtering and dependence on size and energy

The sputtering and ionization mechanisms are mainly defined by the size of the PI, kinetic energy, incident angle, beam fluence (current/pulse), sample nature (organic, inorganic, organometallic) and atomic structural organization (lattice, etc) ${ }^{10-11,24,24,25}$. Fundamental work by Sigmund and coworkers proposed that sputtering processes may follow 3 general mechanisms, defined by the energy transfer onto the atoms and molecules at the surface upon ion impact ${ }^{25-26}$. These mechanisms models are: 1) thermal spiking $\left.(1 \mathrm{eV}>)^{25}, 2\right)$ direct recoil $(>20 \mathrm{eV})^{27,28}$ and 3$)$ the linear collisional cascade $(1-20$ $\mathrm{eV})^{25-26}$. This is also reference as the "hardness" of the impact, or whether it involves elastic or inelastic collisions as the probe interacts with the surface material. They proposed that sputtering by atomic projectiles generally fall in the linear collisional 
cascade model ${ }^{25-26,29}$. This model is based on Newtonian mechanics, rooted in a binary collision approximation model; emission of material occurs due to the momentum transfer from the elastic collision of the incident ion with the surface, making it a purely kinetic processes ${ }^{25-26,29}$. Energy transfer in the linear-impact cascade is based on knockon transfers per binary collision (billiard-ball effect). In this type of energy-transfer process, molecules rarely escape prior to fragmentation as the impact cascade propagates $^{25-26}$. Thus, emission of intact molecules requires less energetic processes, where material in the excited region moves in a concerted manner ${ }^{24,29}$. This can result in the escape of matter at key energies, without the occurrence of fragmentation as all components of the molecule are homogenously energized.

As opposed to elastic processes, inelastic collisions from projectile impacts can induce electronic excitation, resulting in the formation of localized heat (or what is defined as a thermal spike model $)^{30}$. Sputtering of this nature is regarded as kinetically assisted potential sputtering, where the initial impact to generate the concerted, non-linear motion leading to emission contributes energy to generate the thermal spike ${ }^{30-31}$. Factors influencing the occurrence of such elastic or inelastic collisions have been strongly correlated to the projectile cross-section, the energy per constituent distributed on the projectile upon impact (if considering clusters), and the molecular forces holding these projectiles together ${ }^{32,33,20}$. 


\section{Initiation}

1. Atomic Projectile Binary Linear Collisional Cascade

- Atomic Projectile Incident Impact Energy: 25-50eV/projectile

- PI Source Voltage: 5-30k

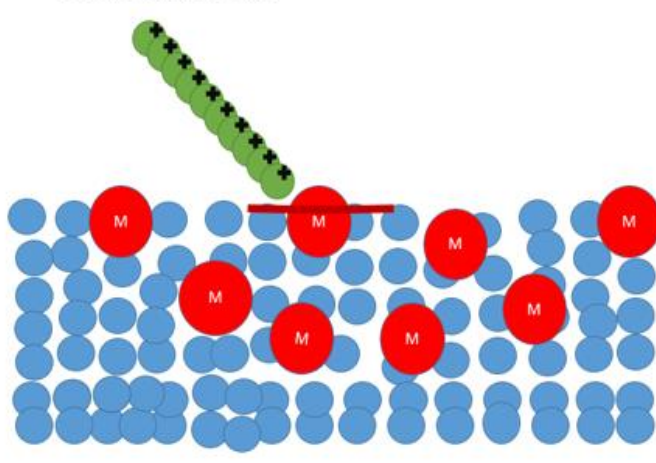

1. Massive Cluster Impact Induced Thermal Spike/Shock Wave. - Cluster/Massive Cluster Incident Impact Energy: 1 $>$ eV/nucleon - PI Source Voltage: $18-30 \mathrm{kV}$.

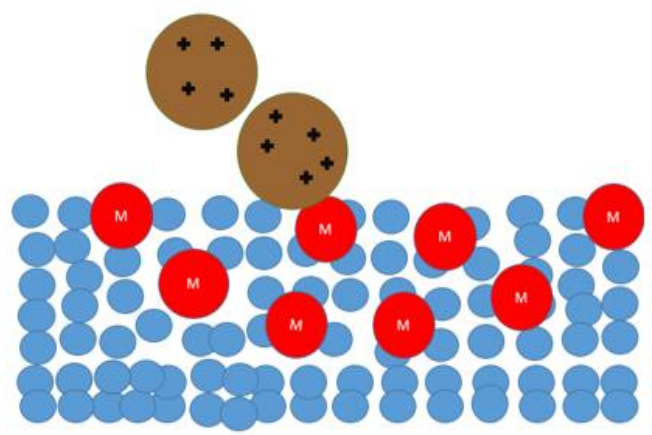

2. Propagation

2. Start of Collisional Cascade

Initial binary collisions propagation at surface upon primary ion impact $1-10 \mathrm{e}$ transfer per interaction via collision, enough to cause excitation of affected species, promoting depth \& lateral propagatio

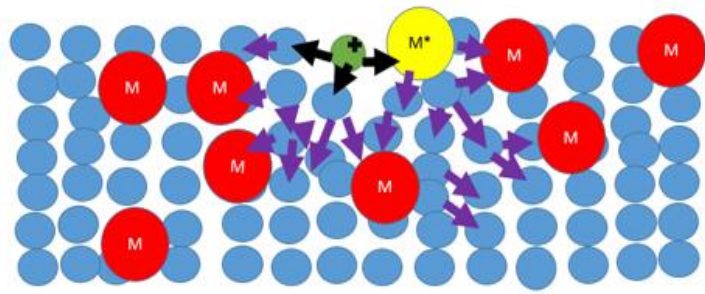

2. Start of Thermal Spiking-Thermal Excitation Due to Formation of Localized Plasma

- Projectile Kinetic Energy Deposited on First Few Monolayers

: Larger Cross-Section Results in More Surface Damage

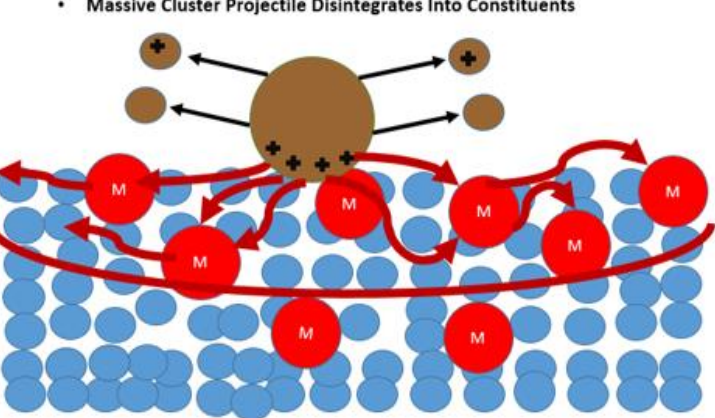

\section{Conclusion}

3. Propagation of Binary Collisions Beyond the Surface (Sub-surface cascade expansion) Heavy fragmentation of molecules due to high energy \& damage

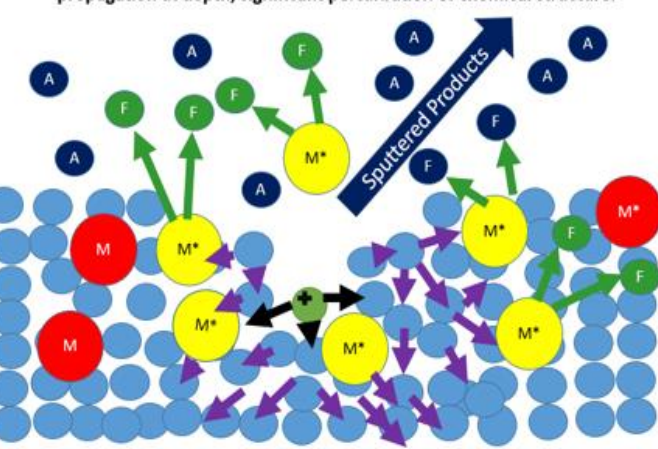

3. Thermal Spike Desorption:

Thermal Excitation Induced "Implosion" following plasmon - Shock-Wave from Projectile Propagates (Vibrational Excitation)

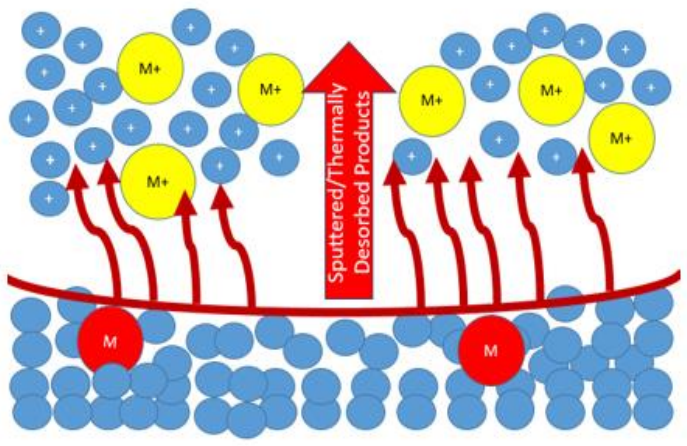

Figure 3. Comparison of sputtering mechanisms based on projectile size. Smaller projectiles engage in a binary collision cascade, while larger projectiles generate localized surface plasma's leading to thermal spiking. 


\section{Secondary Ion Generation Mechanisms}

Whereas the defining factors for sputtering models are fixed, SI generation models are substantially greater in variety ${ }^{24}$. This is rooted in the complexity of ion-surface interactions. Generally, SIs are mainly single charged and angular distributions are influenced by the incidence angle of the ion, and energy distributions generally fall within a Gaussian distribution between $2-5 \mathrm{eV}$. Secondary ion formation depends on the electronic properties of the parent atom/molecule, the electronic properties of the substrates surface, the subsequent polarity of the ions formed, and the type of PI source used. The bond breakage model (Slodzian) suggest that atomic SI species follow from fragmentation and emission, followed by charge transfer processes immediately after ${ }^{34,35}$. The electron tunneling model $(\mathrm{Yu})$ describes SI generation from metallic materials. This model states SI's are generated via resonance charge transfer processes, based on the availability of valence electrons in metals ${ }^{36,37}$. The Langmuir-Saha local thermodynamic equilibrium model (LTE Formalism) describes the ionization processes as a result of localized thermodynamic excitation, generally used to describe sputtering with atomic beams, but is applicable to contexts of polyatomic projectile impacts ${ }^{29,30}$.

Models exclusive to molecular ion generation are more limited, and tend to overlap with MALDI-based observations. Rabalais's model proposes that molecular species are emitted in a charged state, and may become neutralized or fragmented within

a certain range from the surface as a way for molecule to dissipate internal energy ${ }^{38,39}$. Since the probability for neutralization and fragmentation of SI's is influenced by the excitatory energy deposition, projectiles with lower energies such as polyatomic species are preferred over atomic species. Benninghoven's precursor model states an energy 
gradient is generated around the impact site ${ }^{40,16}$; the impact energy induces fragmentation of existing molecules at the site, however molecular ions further away are provided an excitatory energy large enough to promote the ion emission without fragmentation ${ }^{17}$. Larger projectiles have been found to have increasingly significant impact cross-sections, thus removing larger material from the surface. However, the energy distribution in these impacts is more discrete in nature and evenly distributed on the surface as a function of $\operatorname{size}^{33}$. For large projectile impacts, MALDI-based models have been utilized were the emission of intact molecular species is a result of the concerted motion characteristic of a thermal spike process ${ }^{12}$. Relaxation of the thermodynamically excited surface coincides with the generation of a plume of electrons, protons and emitted ions. Work by Michl and Cooks has also brought into consideration the occurrence of the thermodynamical spike as a step-wise process, where PIs are generated through a collisional cascade, and additional ions are emitted afterwards due to thermal excitation ${ }^{41,42}$. However, occurrence of these spikes and emitted species depend on the matrix, as organic and inorganic samples will require different energies to create a thermally excited $\operatorname{area}^{41}$. Delving deeper into these localized thermodynamic spikes, there is a variety of recombination and energy isomerization reactions ${ }^{43,44,45}$. More recent work by Delcorte has summarized the occurrence of these plumes during polyatomic projectile impacts ${ }^{46,47}$. Photon-induced fragmentation (electronic excitation) electron-induced fragmentation, anionization, cationization, and neutralization processes are the most prevalent outcomes within these plumes. Of these processes, anionization and cationization are the only ones of interest as they result in the generation a molecular ion. Nonwithstanding, cationization is more prevalent, as positive charged SIs are more stable when compared to their negative 
counterparts ${ }^{47}$. In the cationization/anionization process, a cation is co-emitted along with a neutral molecule with equal momentum during the sputter process ${ }^{46}$. The secondary positive molecular ion species may interact with another sputtered neutral (direct interaction) or be sputtered as an excited neutral which emits electrons during its relaxation process as a product of ion-ion/ion-neutral interactions. The usage of polyatomic and cluster projectiles has been found to promote the formation of energetically gentle plumes. Subsequently, recombination mechanisms like anionizations and cationization processes become dominant in the plumes ${ }^{47}$. Additionally, larger projectiles evacuate greater amounts of materials from the surface, increasing the probability of ion generation as there are both more species to transfer charges and excited neutrals emitted.

\section{Theoretical Tools for Examination of Projectile-Surface Interactions}

In order to extend these models, $\mathrm{X}$ been performed new theoretical studies, based on molecular dynamics (MD) ${ }^{48}$. MD simulations can also be enhanced by utilizing $a b$ initio methods, for interactions that are very complex such as hydrodynamic effects illustrating massive cluster impacts ${ }^{49,50}$. Common variables in the simulations are the cluster nature, surface material, projectile velocity, and incidence angle. In atomic projectile impact simulations, the initial impact interactions occur at the femtosecond scale, and mostly occur around the perturbance caused by the energy of the incident ion ${ }^{48}$. The transferred energy exceeds the nuclear forces holding together the surface atoms and molecules. For atomic models, this stage can be elucidated by employing binary collisional approximation (BCA) according to Sigmund's sputtering mode ${ }^{24}$. Programs commonly utilized to model these processes are Stopping Range of Ions in Matter, and 
Marlowe $\mathrm{e}^{51},{ }^{52}$. The former is far more prevalent, and has been found to be able to model some cluster systems, however it has difficulties simulating the full life-span of the projectile and surface following PI impact ${ }^{53}$. Fully elucidating a cluster impact however still remains challenging due collective effects from many-body interactions and concerted motions of atoms characteristic to a thermal spike mechanism ${ }^{53}$. In molecular dynamic calculations the forces between different constituents of the system are computed based on positions and interaction potentials ${ }^{50,53}$. Hamiltonian equations of motion are integrated to produce information regarding the velocity and direction of the particle per time step $^{48,53}$. MD models also provide behavior information for a range of energies, bond breakage/formation processes, and the morphology of the interaction ${ }^{50}$.

\subsection{Atomic Ion Sources}

Primary Ion (PI) columns initially were based on monoatomic ion sources, which were generally constricted to analyzing fragments or elemental compositions ${ }^{10-11}$. This was due to the violent collisional cascade ionization mechanism related to these projectiles energy, around $\sim 50 \mathrm{eV} /$ nucleon, far beyond thermal spiking region ${ }^{24,34}$. Due to the collisional cascade mechanism, deeper layers of the sample generally become damaged, making any depth-specific molecular data difficult to obtain ${ }^{11,25}$. These atomic probes generally utilize metal or noble gases as projectiles, such as gallium, cesium, indium, xenon, argon, oxygen, bismuth and gold ${ }^{10}$.

The types of PI emitters/sources divided in Field Emission Sources(FES), Liquid Metal Ion Sources (LMIS), Electron-Gas Impact Sources, Thermal Ionization, plasmadesorption products $\left({ }^{252} \mathrm{Cf}\right.$ PD) and DC-Glow Discharge (Duoplasmatrons) ${ }^{10}$. Field 
emission sources involve a filament coated in a metal eutectic, from which the ionized metal is emitted ${ }^{54}$. Metals become ionized via an electron-metal electron exchange process driven by quantum tunneling ${ }^{54-55}$. Such a process is ignited by applying a high potential across the filament, leading to the formation of an electrified interface (Taylor Cone) for ion emission ${ }^{56} \mathrm{Cs}^{+} \mathrm{PI}$ beams were the first to be used from FES, demonstrating a good amount of source brightness with stable current output, reasonable energy spread for focusing and decent life-times ${ }^{55}$. Further work optimized the basic geometry, materials and emitter properties for a variety of metals ${ }^{57,58}$. Duoplasmatrons were some of the earliest sources as well, employing principles similar to those in inductively coupled plasma (ICP) sources ${ }^{59}$. A high-velocity stream of oxygen, halogen or noble gas is impacted by electrons from a cathode-anode discharge, generating a ionized gas area that is shaped by a magnetic field, creating a fine plasma ${ }^{59}$. These sources are known to work well with oxygen $\left(\mathrm{O}^{+}, \mathrm{O}^{-}\right)$, generating ions with excellent reactivity ${ }^{59}$. Gas-Electron Impact sources employ a gas jet were expansion occurs, and the stream is ionized by electrons from an EI source perpendicular to the path of the gas ${ }^{60}$. The electrons are trapped and ionize the residual gas; ions are extracted and accelerated. This type of source has been used to generate very stable $\mathrm{Ar}^{+}, \mathrm{O}^{+}$, and $\mathrm{Kr}^{+}$beams, yet with a very wide energy spread inherent to the mechanism of ion generation ${ }^{5,15}$. Currently, many of these sources are still utilized in commercial instrumentation (e.g., CAMECA ion microprobes); however their use has been largely eclipsed by polyatomic projectiles for molecular analyses. Thermal Ionizers were reserved to the usage of metal salts with low melting points such as cesium iodide, where the salt housed in a reservoir was heated and directed by an electrostatic potential on a fritted film, where the ions are emitted from ${ }^{18}$. 
This source still sees plenty of commercial usage in elemental analyses due to its ease of focusing, down to tens of nanometers in instruments such as the Cameca "nanoSIMS" product line $61,62,63$.

One of the more unorthodox sources is the usage of ${ }^{252} \mathrm{Cf}$ isotopes for Plasma Desoprtion Mass Spectrometry (PDMS), a technique analogue to SIMS ${ }^{64}$. In this source fission products are used to bombard the sample ${ }^{64}$ followed by the generation of SIs and neutral species. The high energy of the fission fragments is able to generate significant intact molecular ion yields, desorb intact molecular biological species and is considered the entry point into the ionization of surface-bound biomolecules ${ }^{64,65}$.

\subsection{Polyatomic and Cluster Ion Sources}

The start of the polyatomic projectile era was driven by several needs of the SIMS community, such as enhancing secondary molecular ion yields. Prior work by the charged particle physics communities proved decreased surface damage cross-sections were obtained when using cluster projectiles, as well as non-linear enhancements in secondary emitted material ${ }^{11,32}$. H.H. Andersen's work in the 1970 s was the first evidence of nonlinear enhancements, where sputtering yields for $\mathrm{Te}^{+}$and $\mathrm{Te}_{2}{ }^{+}$were compared on $\mathrm{Au}, \mathrm{Ag}$ and Si substrates ${ }^{66}$. A precision-measured piece of quartz was attached to a microbalance; this allowed for the measurement of volume of sputtered material per projectile bombardment time period ${ }^{66}$. The results showed that non-linear enhancements were detected in all substrates bombarded, with sputtering ratios between $\mathrm{Te}_{2}{ }^{+} / \mathrm{Te}^{+}$spanning from 1.30 to 2.27 . Further work compared ions from monoatomic species based on weight to evaluate if the same non-linear enhancements were observed, with a linear trend still present ${ }^{67}$. The ratio of molecular to atomic SI species was also measured 
utilizing $\mathrm{Te}_{2}{ }^{+} / \mathrm{Te}^{+}$and $\mathrm{Se}_{2}{ }^{+} / \mathrm{Se}^{+}$, with results supporting the occurrence of non-linear enhancement in secondary molecular ion generation as opposed to secondary atomic ions ${ }^{67}$.

During the late 1980's, Appelhans and coworkers devised a gas-electron capture source utilizing sulfur hexafluoride gas $\left(\mathrm{SF}_{6}\right)$ that produces a distribution of $\mathrm{SF}_{6}{ }^{0,-}$ (neutral/anion combined beam) projectiles ${ }^{68}$. This novel type of probe laid the groundwork for comparisons between atomic and polyatomic projectiles. Relative SI sputtering efficiencies $(\varepsilon)$, the number of SI per incident ion impact for a particular $\mathrm{m} / \mathrm{z}$ value per unit time for interrogation of organic substrates (Codeine, Tylenol, etc) were compared between $\mathrm{SF}_{6}{ }^{0}$ and $\mathrm{Cs}^{+}$beams ${ }^{68}$. Analyses were performed with equivalent acceleration and similar instrumental parameters in order to minimize deviation. Results showed that efficiencies for desired $(\mathrm{M}+\mathrm{H})$ ions showed enhancement factors from 9 $(300 \mathrm{~m} / \mathrm{z})$ to $24(137 \mathrm{~m} / \mathrm{z})$ with the $\mathrm{SF}_{6}{ }^{0,-}$ beam, and an increase in LOD by a factor of $\sim 10^{368}$. Evaluation of damage cross-sections stems from comparisons of each projectile's transformation probability, the probability $(P)$ that an atomic or molecular species leaving the surface will be in an ionized state, is defined as $P$

$$
P=\frac{Y_{S}}{\sigma \vartheta_{0} \theta}
$$

where $\mathrm{Y}_{\mathrm{s}}$ is the sputter yield and $\sigma \vartheta_{0} \theta$ is defined as the surface coverage term ${ }^{68} . \vartheta_{0} \theta$ is defined as sample preparation-related variance, which was the same for both samples. The polyatomic projectiles produced 1.8 times the damage per impact compared to the $\mathrm{Cs}^{+}$ions, and a 2.5 times increase in transformation probability for $\mathrm{SF}_{6}{ }^{0,-}$. Theoretical conclusions stated that this projectile exhibited gentler ionization mechanisms compared 
to $\mathrm{Cs}^{+46}$.This transformation probability in itself defines the useful yield, another nomenclature to distinguish between sputter yield (neutrals and ions) and the SI yield ${ }^{68}$.

Work in the late 1980s by M. G. Blain, Della-Negra and E. A. Schweikert also explored the benefits of a polyatomic $\mathrm{Cs}_{\mathrm{n}} \mathrm{I}_{\mathrm{M}}^{\mathrm{q+}}$ beam, by comparing with $\mathrm{Cs}^{+}, \mathrm{Cs}_{2} \mathrm{I}$, and $\mathrm{Cs}_{3} \mathrm{I}_{2}{ }^{+}$projectiles ${ }^{69}$. Additional comparisons included the usage of coronene $\left(\mathrm{C}_{24} \mathrm{H}_{12}{ }^{+}\right)$and its dimers as projectiles. These projectiles were generated by desorpsion ionization by ${ }^{252} \mathrm{Cf}$ fission fragments impacts ${ }^{69}$. Non-linear collision cascades were postulated in this work, defined as a product of a non-linear ionization mechanism favoring molecular ion generation (thermal spike mechanism), as well as non-linear yield enhancements ${ }^{30,40,69}$. The expression for ionization yield enhancement for a single homonuclear (cluster) projectile is defined as

$$
Y_{A_{n}}(E) / n Y_{a}(E / n) \geq 1
$$

$\mathrm{Y}_{\mathrm{n}}(\mathrm{E})=\mathrm{n} \mathrm{Y}_{1}(\mathrm{E} / \mathrm{n})$, where $\mathrm{Y}_{\mathrm{n}}(\mathrm{E})$ is the yield for a projectile of $(n)$ homonuclear constituents and $\mathrm{nY}_{1}(\mathrm{E} / \mathrm{n})$ is the yield for successive monoatomic projectile impacts corresponding to the number of constituents ${ }^{66,69}$. An expanded expression for heteronuclear polyatomic projectiles is also defined. Comparisons took place over a range of energies from 5 to $28 \mathrm{keV}$. SI yields were measured in an event-by-event mode to examine individual projectile-surface interactions. SI yields for different projectiles were obtained and compared, as each individual impact provided a complete data set per projectile type. Comparison of $\mathrm{Cs}^{+}$and $\mathrm{Cs}_{n} \mathrm{I}_{\mathrm{M}}{ }^{\mathrm{q}+}$ resulting yields on a phenylalanine target display an enhancement factor up to 10 for molecular ion generation $(164 \mathrm{~m} / \mathrm{z})$, influenced by the projectile's impact energy. Comparisons of $\mathrm{Cs}_{\mathrm{n}} \mathrm{I}_{\mathrm{M}}{ }^{\mathrm{q}}$ and $\mathrm{nC}_{24} \mathrm{H}_{12}{ }^{+}$ 
$\left(\left(\mathrm{M}^{+} / 2 \mathrm{M}-\mathrm{H}^{+}\right)\right.$against $\mathrm{Cs}^{+}$impacts on a gold target showed that the largest, non-linear enhancements in yield were found when utilizing the largest projectiles $\left(\mathrm{Cs}_{3} \mathrm{I}_{2}{ }^{+}, \mathrm{C}_{48} \mathrm{H}_{26}{ }^{+}\right)$, with enhancement factors for of 5 and 4.5 for $\mathrm{Au}^{-}$SIs, respectively ${ }^{69}$. Oxygen cluster $\mathrm{O}_{2}{ }^{+}$ projectiles were also utilized in these efforts, being a highly useful tool for surface sputtering ${ }^{4}$. The $\mathrm{O}_{2}{ }^{+}$projectiles are still frequently used in many SIMS instrumentation, particularly for the CAMECA product lines as sputtering beam with the best focusing potential to date. However, its usage as a surface cleaning and eroding beam has been eclipsed by massive gas clusters, which have significantly enhanced sputter yields, penetration and create no reactive species.

Further work of high relevance was also performed by researchers at Institute of Nuclear Physics in Orsay by Della-Negra and co-workers. They focused on the study of novel cluster and polyatomic sources, in order to further evaluate the occurrence of nonlinear enhancements by utilizing a variety of projectiles, both metallic and organic ${ }^{70}$. Other aims involved differences in terms of the ionization mechanisms and subsequent yields from organic, inorganic and composite materials, and post-impact projectile fate ${ }^{71}$. A platform for studying these interactions at very high energies $(\mathrm{MeV})$ and performing comparisons between projectiles, also known as the ORION project was also created ${ }^{70}$. These studies also were one of the first to employ an event-by-event mode for SIMS, which will be discussed more in depth in Chapter 3. Their work provided valuable information regarding the distributions of $\mathrm{Au}_{n}{ }^{\mathrm{q}}, \mathrm{Cs}_{\mathrm{n}} \mathrm{I}_{\mathrm{M}}{ }^{\mathrm{q}}$, and $\mathrm{C}_{\mathrm{N}}{ }^{+\mathrm{q}}$ projectiles from a variety of source types such as LMIS, electron-gas impact and solid substrate sputtering ${ }^{72} \cdot \mathrm{Au}_{\mathrm{n}}{ }^{\mathrm{q}+}$ distributions were mapped utilizing the ORION platform based on TOF measurements on the PI column utilizing a series time-pulsed of deflector plates. A 
distribution of $\mathrm{Au}^{+}, \mathrm{Au}_{2}{ }^{+}, \mathrm{Au}^{+}, \mathrm{Au}_{4}^{+}$, and $\mathrm{Au}_{5}^{+}$projectiles was measured from a $\mathrm{Au}-\mathrm{Mn}$ eutectic LMIS ${ }^{73}$. These projectiles were compared amongst each other at slow and swift velocities, significant gains in yield were found with increasing projectile size in particular for organic targets ${ }^{73-74}$.

A primary aspect of this work was focus on the use of larger cluster projectiles, including the first instance of fullerene projectiles ${ }^{75}$. Briefly, a compressed pellet consisting of $90 \% \mathrm{C}_{60}$ was impacted by a $\mathrm{Cs}^{+} \mathrm{PI}$ beam, generating $\mathrm{C}_{60}{ }^{-}$species. These were charge compensated via collisions following high-energy acceleration inside of a $\mathrm{N}_{2}$-filled cylindrical gas shell, stripping the projectiles of electrons. $\mathrm{C}_{60}{ }^{+}$and $\mathrm{C}_{60}{ }^{+2}$ and $\mathrm{C}^{+70}$ species were detected from this source type and utilized as primary projectiles for the evaluation of low $(\mathrm{keV})$ and high $(\mathrm{MeV})$ energy impacts, as well as comparisons with other novel cluster projectiles at the time $e^{75,72}$. Comparisons between projectiles $\left(\mathrm{Au}_{(1-5)}\right)^{+1}$, $\mathrm{C}_{60}{ }^{+}, \mathrm{C}_{70}{ }^{+}$) at low velocities on two targets were performed at $20 \mathrm{kV}$ acceleration. A target made of heterogenous layers as well as a homogenous phenylalanine surface were completed to compare surface penetration and SI yields based on the target and projectile utilized. Results showed that gold clusters penetrated roughly 3 times deeper as opposed to fullerene-based projectiles, with a lower emission yield on the layered heterogenous target and an overall lower emission yield on the phenylalanine target ${ }^{76}$. Mechanism observations proved that the larger impact cross-section and lower energy/nucleon meant the $\mathrm{C}_{60}{ }^{+}$projectile deposited most of its energy on the surface upon impact, reducing its overall penetration and enhancing surface-derived emissions while reducing depthdamage. Work by Van Stipdonk and coworkers compared the relative yields of $\mathrm{Ga}^{+}, \mathrm{C}_{60}{ }^{+}$ and $\mathrm{Cs}_{3} \mathrm{I}_{2}{ }^{+}$employing a novel discrete projectile mapping approach, based on similar 
principles to the previously discussed event-by-event mode, with the inclusion of a secondary electron detector for localization of individual impacts ${ }^{77}$. In this approach, the PI beam is considerably collimated and pulsed at a very high rate $\sim(500 \mathrm{~Hz})$, resulting in a very small beam fluence (10-100 impacts/s) ${ }^{77}$. Results noted increases in overall SI yields increases with both projectiles, with $\mathrm{C}_{60}{ }^{+}$generating SIs with an efficiency two times greater than the CsI cluster beam. However, $\mathrm{C}_{60}{ }^{+}$also generated a significant increase of cluster/molecular ion species $(\mathrm{CsI})_{2} \mathrm{I}^{-}$and $\mathrm{CsI}$, with an enhancement factor 4 times greater of $(\mathrm{CsI}) \mathrm{Cs}^{+}$projectiles, and over 50 times greater than $\mathrm{Cs}^{+}$and $\mathrm{Ga}^{+} 77$. However, regardless of the evident benefits and surmounting body of work to support the usage of metallic and fullerene cluster projectiles the complexity in generating these type of PI beams and the difficulty in subsequent focusing made them impractical for some time.

Gillen and coworkers used a $\mathrm{SF}_{5}{ }^{+}$source for chemical mapping of thin organic films in comparison to an $\mathrm{Ar}^{+}$source. Based on an improved source design from Delmore's work, an $\mathrm{SF}_{5}{ }^{+}$beam was focused down to a $\leq 50 \mu \mathrm{m}$ spatial resolution and operated at voltages from $3-15 \mathrm{kV}^{78}$. The $\mathrm{SF}_{5}{ }^{+}$beam provided some of the expected benefits such as increased molecular ion yields, effectively perform molecular ion imaging of the target surface with significant SI and sputter yielded increases. During depth profiling it was able to effectively remove damaged layers to continuously expose fresh, unperturbed material ${ }^{78}$. It was capable of restoring surfaces damaged by the $\mathrm{Ar}^{+}$ source, restoring molecular ion signals for molecular ion species with the $\mathrm{Ar}^{+}$was unable to sustain for more than a few surface monolayers. This effect of molecular ion signal restoration was noted in thin organic films, on bulk biological materials, and on polymer- 
based materials ${ }^{78}$. Theoretical and mechanistic insights showed that the projectile generated an expected non-linear cascade, generating SIs within the thermal spike model energy's threshold. Simulations utilizing SRIM proved that while the surface impact cross-section was greater, all energy was immediately dissipated in the upper monolayers, with very minimal depth-specific damage ${ }^{52,78}$.

The early and mid-2000's found these projectiles being revisited for the goal of utilizing some of these sources in order to do high spatial resolution molecular ion mapping, and their subsequent introduction into the instrument market ${ }^{11}$. Designs were optimized to reduce the probe size, as well as maximizing generation of cluster species, source brightness and simplifying/automating operation. Companies like IONTOF (Germany) and IONOPTIKA (United Kingdom) played an important role in the popularization of cluster SIMS sources, as they made these sources commercially viable. $\mathrm{SF}_{5}{ }^{+}$and $\mathrm{Au}$ LMIS were the first type of cluster probes redeveloped for imaging purposes to be utilized as a sputter/analysis dual-beam approach and introduced to the research community ${ }^{79}$. Original work for imaging applications compared $\mathrm{Au}^{+}$and $\mathrm{Au}_{3}{ }^{+}$projectiles, utilizing an innovative design from IONOPTIKA, to produce high, stable source brightness and the potential for submicron lateral resolution ${ }^{79,80}$. $\mathrm{Au}_{3}{ }^{+}(591 \mathrm{~m} / \mathrm{z})$ was found to generate molecular ion yield by an order of 60 when compared to $\mathrm{Au}^{+}$for the purpose of chemical mapping while enhancing generation and retention of molecular ion species, and reducing unwanted depth penetration ${ }^{81}$. Shortly after, IONTOF introduced the Bi-Mn LMIS, with an equally promising lateral resolution and source brightness as the Au-LMIS ${ }^{82}$. However, this LMIS is capable of generating polyatomic projectiles such as $\mathrm{Bi}_{3}{ }^{+}$and $\mathrm{Bi}_{3}{ }^{+2}$ more efficiently than $\mathrm{Au}-\mathrm{Ge}$ or $\mathrm{Au}-\mathrm{Si}$ sources ${ }^{82-83}$. Owing to its larger 
size, $\mathrm{Bi}_{3}{ }^{+}(627 \mathrm{~m} / \mathrm{z})$ generated even greater SI yields and had a characteristically higher sputtering and ionization efficiency which has placed it as one of the best commercially available beams for static SIMS molecular surface characterization and imaging ${ }^{84}$. These projectiles are able to generate molecular ions in masses of excess of $1 \mathrm{kDa}$, opening the door for lipidomics and metabolomics of biological targets at the nanoscale ${ }^{83}$.

Around this time, an improved version for generation of $\mathrm{C}_{60}{ }^{+}$projectiles was introduced by IONOPTIKA in conjunction with Vickerman ${ }^{85}$. The $\mathrm{C}_{60}{ }^{+}$source, based on electron-impact ionization of gas-phase fullerene showed increased brightness, stability for extended operation, and facilitated focusing for its usage in chemical mapping and localization. From this development, $\mathrm{C}_{60}{ }^{+}$has proven itself to be efficient in both static and dynamic SIMS applications, particularly for biological applications ${ }^{86,87} \cdot \mathrm{C}_{60}{ }^{+}$is capable of acting as "hybrid" beam where it can be used to sputter and/or analyze sample layers when doing any form of 3D profiling ${ }^{86,88}$.Until recently, the effective spatial resolution of $\mathrm{C}_{60}{ }^{+}$is unsuitable for nanoscale observations in static SIMS analyses, with most commercial versions threading in the $1-10 \mu \mathrm{m}$ range. A very comprehensive review from Winograd shows the advances of this source in the context of nanoscale analysis, showing that new versions can obtain spatial resolutions of $\sim 200 \mathrm{~nm}$ when operated in DC mode ${ }^{15}$. 


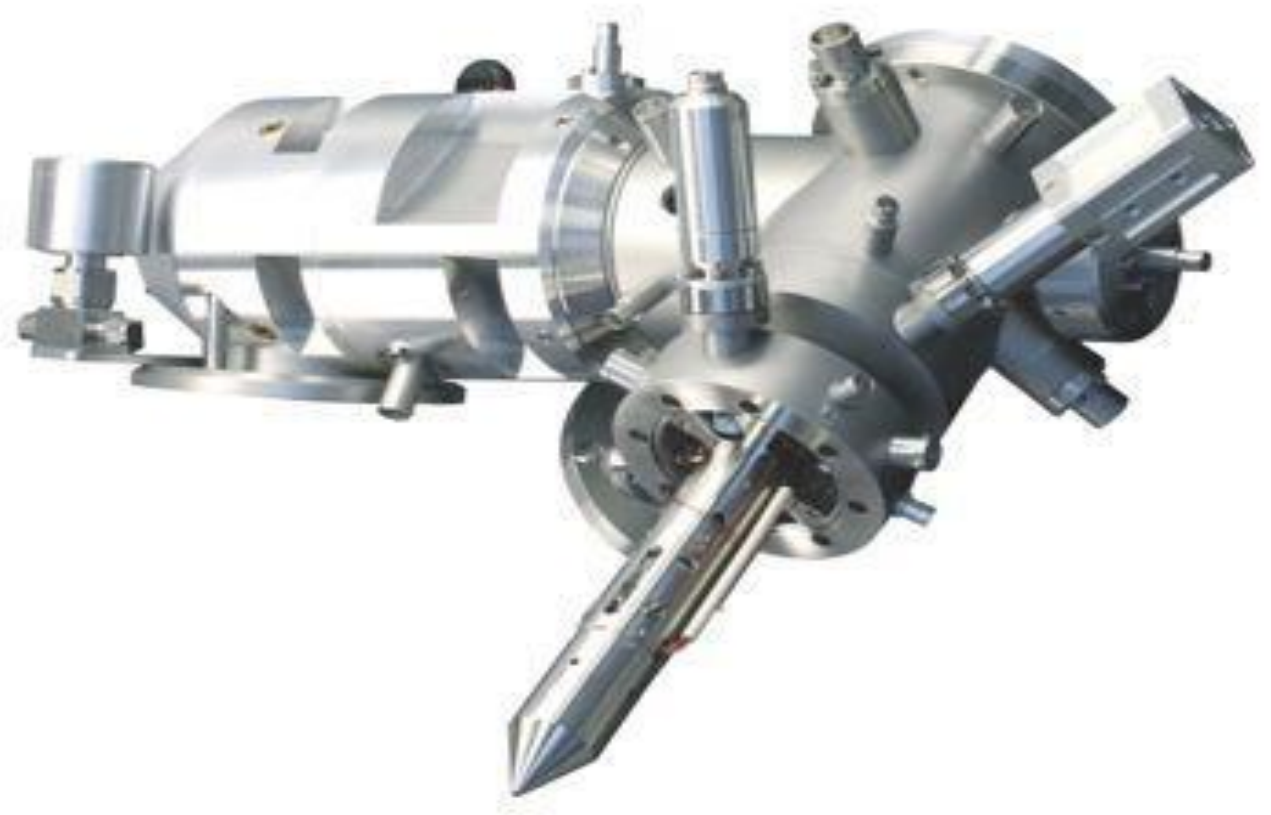

Figure 4. IONTOF $20 \mathrm{kV}$ C60 Primary Ion Column. Reproduced with permission of IONTOF GmbH (Munster, Germany).

It must be highlighted that the choice of mass analyzer has been found to also have very important implications in the operation of these instruments. Most conventional molecular application-oriented SIMS instrumentation utilize time of flight (TOF) analyzers, yet these lack the resolving power required to perform exact chemical identification of molecular ions from complex organic substrates, such as biological surfaces ${ }^{11}$. Previous work has shown that the integration of a $\mathrm{C}_{60}{ }^{+}$cluster source from IONOPTIKA in conjunction with an ultra-high resolution mass analyzer such as a FTICR MS can greatly expand the capabilities of exact chemical identification of generated molecular ions ${ }^{89,90}$. This instrument has also been compared to a commercial state-of- 
the-art instrument (TOF-SIMS ${ }^{5}$, and has significantly more effective at resolving and subsequently identifying a variety of lipid species ${ }^{91}$.

\subsection{Massive Clusters and Charged Nanodroplets}

In the early 90's, Mahoney and coworkers investigated the application of nonvolatile, charged droplets generated via vacuum electrospray as SIMS projectiles ${ }^{92}$. These involved the usage of glycerol doped with electrolytes such as ammonium acetate, in order to generate massive, multi-charge projectiles for the generation of molecular ion species. Massive Cluster Impacts (MCI) were found to generate little to no fragmentation, due to the low energy/nucleon $(0.6 \mathrm{eV} / \mathrm{n})^{93}$. This allows MCI to generate significant molecular ion signals in excess of $2 \mathrm{kDa}$ corresponding to intact peptides ${ }^{94}$. This projectile however was abandoned shortly after due to inherent difficulties in producing consistent results, due to variables such as voltages, flow rate, emitter material and dimensions, and ionization polarity. It was recently revived by Williams and coworkers, where they combined this source with a high resolution secondary in microscope, obtaining spatial resolution below $10 \mu \mathrm{m}$ while generating intact peptide SI's ${ }^{94,95}$.

Additional work in utilizing large charged droplets at hypervelocities was also explored by other groups ${ }^{96}$. A platform similar to the ORION system, known as the Multiply Charged Macromolecular Accelerator (MUMMA), was developed in the mid 90 's to evaluate the feasibility of electrosprayed macromolecules as PI beam sources ${ }^{96}$. Work with this instrument focused in measuring yields based on event-by-event photon measurement per projectile impact, in conjunction with surface modifications from these impacts. This was driven by the results of the work at IPNO, with an interest on whether 
utilizing multiply charged species such as proteins and other biomolecules could provide further significant enhancements of molecular ion yields while reducing unwanted overpenetration or deposition ${ }^{96,97}$. Projectiles used in this work included bovine insulin (5.3 $\mathrm{kDa})$, hen-egg lysozyme (14.3 kDa) and apo-myoglobin $(16.9 \mathrm{kDa})$, containing varying number of charges to evaluate the effects of acceleration ${ }^{96,98}$. PIs were chosen based on a quadrupole mounted on the ion optics column to allow surface impact with a specific charge state prior to pre-impact acceleration. Conclusions from this work showed that the yield enhancements were noted as a function of increasing velocity, yet they were significantly lower than expected ${ }^{97-98}$. Effects of impacts on a variety surfaces displayed non-reproducible modifications, with varying degrees of surface and depth damage.

Sometime later, during the rise in popularity of cluster sources in commercial SIMS instrumentation, Boneau and Della-Negra from IPNO performed an expanded study into the full projectile distribution generated by Au-LMIS's ${ }^{99}$. Taking an existent Au-LMIS design, the extraction apertures (lens) were modified to generate geometry favoring charged nanodroplet generation. The characterization studies involved profiling the entire distribution, with a particular interest in larger projectiles (n/q>30), where they found $\mathrm{Au}_{100(\mathrm{n})}{ }^{\mathrm{q}(\mathrm{n})}$ clusters. These projectiles in particular have a ratio of $\mathrm{n} / \mathrm{q}=100 \sim 120$, with the $\mathrm{q}=4,5$ accounting for $45-50 \%$ of the distribution, making them the most commonly generated projectile for this specific distribution ${ }^{99}$. These projectiles were also found to induce very little surface damage, having a damage cross section substantially lower than that of $\mathrm{Au}^{+}-\mathrm{Au}_{9}{ }^{+}$(Figure 5) while maintaining continuous molecular ion signal compared smaller projectiles ${ }^{99}$. Early work with this type of projectile utilizing an orthogonal time of flight detector proved that it could generate intact molecular ion 
species for masses up to $1.8 \mathrm{kDa}$, but suffered heavily in terms of focusing with a spot size of $7 \mathrm{~mm}^{99}$. Interest in this type of source was resurrected by the Schweikert group, where the goal was to pulse hypervelocity $(\sim 140 \mathrm{kV})$ individual projectiles for impact as opposed to using a pulsed beam. Using this approach, the spatial resolution is limited by the perturbed volume from where the SI's are generated ${ }^{100,101,102}$. Analyses of model biological systems was performed with this instrument, generating intact lipids beyond $1,000 \mathrm{~m} / \mathrm{z}$ while retaining spatial resolution in the order of tens of nanometers ${ }^{103}$. The SI yields increased by 3 orders of magnitude, when compared to $\mathrm{Au}_{3}{ }^{+}$and $\mathrm{C}_{60}{ }^{+} \mathrm{PI}$ sources as well as a greater number of molecular ion species detected overall ${ }^{101}$.
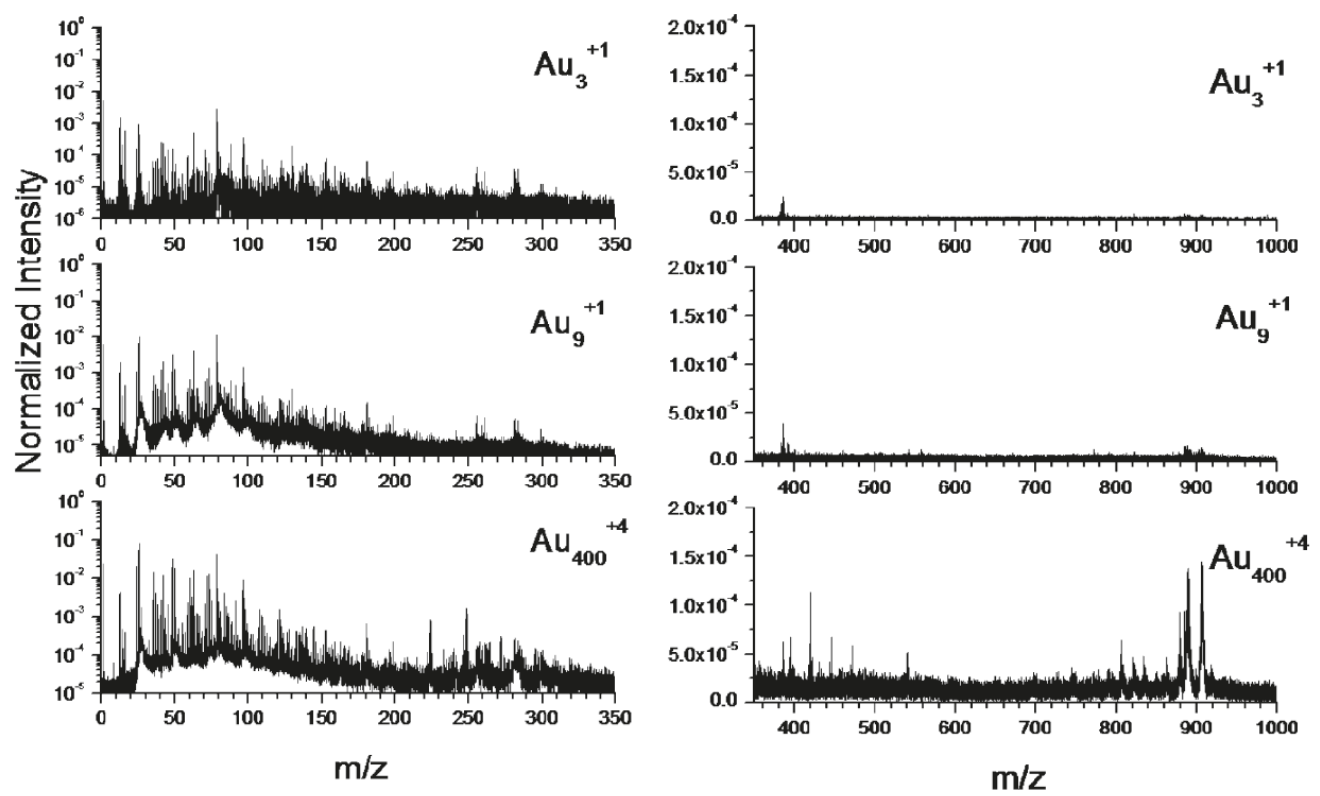

Figure 5. Comparison of negative ion mode spectra for high-energy $\mathrm{Au}_{3}{ }^{+}, \mathrm{Au}_{9}{ }^{+}$, and $\mathrm{Au}_{400}{ }^{+4}$ projectiles on a native rat brain section. Note the significant increase in overall SI yield (intensity) and greater molecular ion intensity and variety, and generation of intact molecular lipids $\left(\sim 900 \mathrm{~m} / \mathrm{z}\right.$ region) when employing $\mathrm{Au}_{400}{ }^{+4}$. 


\section{Reprinted with permission from FFL et al (2012). Copyright 2012 American Chemical Society.}

A relative newcomer in the world of cluster SIMS, argon gas cluster sources date back to mid 2000's, developed by the Matsuo and $\mathrm{co}^{104}$. In their early work, they outlined the performance of varying size Ar clusters with respect to the bombardment effects such as sputtering yields, total perturbed area, impact energetics and focusing potential ${ }^{104-105}$. Gas clusters ion beams are generated by pushing a highly pressured gas through a desorbing nozzle, where neutral gas clusters are formed. These clusters interact with electrons generated by an orthogonally-positioned electron source, where the ionization of these clusters takes place. The ionized projectiles then accelerated by extracting optics up to tens of thousands of kilovolts, and mass-filtered in a $90^{\circ}$ magnet sector with a field of generally $\sim 1 \mathrm{~T}^{104}$. By altering the entrance angle of the ion with respect to the magnet, the geometry changes, allowing generally only significantly large projectiles through the magnetic filter. Modern commercial designs like that from IONTOF (presented in Figure 6) utilize compact Wien Filter designs in order to reduce instrumental footprint. From this 
point the argon clusters are focused and subsequently pulsed for surface rastering.

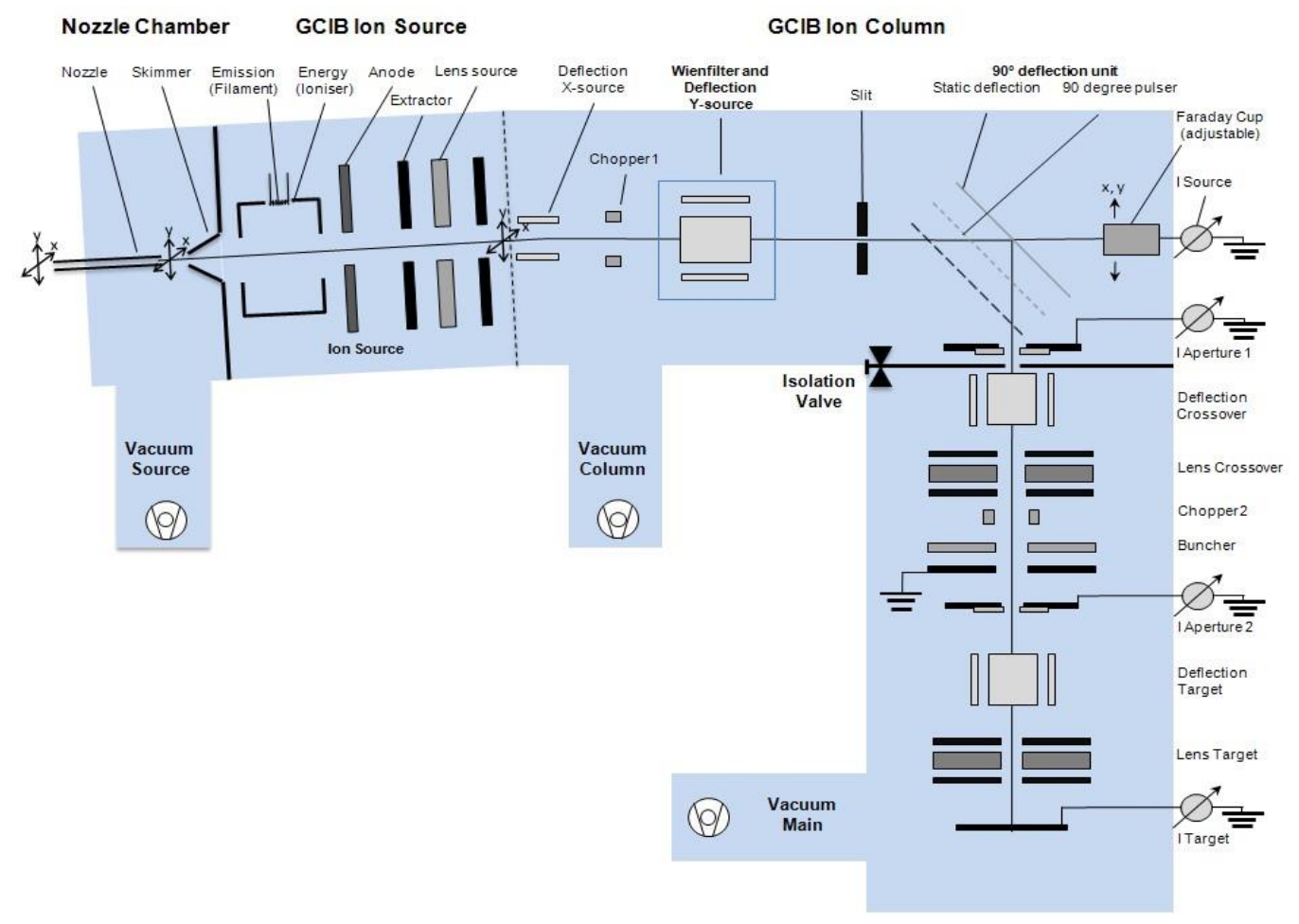

Figure 6. Schematic of an IONTOF Argon Gas Cluster Primary Ion Column (ArGCIB). Reproduced with permission of IONTOF GmbH (Munster, Germany).

The sputtering capabilities of these projectiles have been proven to be directly related to the initial kinetic energy and cluster size. It was also demonstrated that $\mathrm{Ar}_{1000}{ }^{+}$ clusters were the most capable in terms of sputtering, with ion intensities and corresponding sputtering yields falling off beyond this cluster size ${ }^{104}$. Ar-GCIB has become the industry's workhorse in terms of molecular analysis where retention of deeper structure and acquisition of surface data ${ }^{106,107,108}$. As mentioned in the introduction, dual-beam instrumentation has gradually become the standard in SIMS instrumentation, with the GCIB quickly rising to be the go-to sputter beam for organic applications ${ }^{109,110}$. This beam can also act as an analysis beam at the microscale, having been used to study plenty of organic materials as well as inorganics thanks to its 
excellence in delivering excellent ionization yields in its own merit. Current research involving this source type involves generation of clusters well over 1000 constituents $\left(\operatorname{Ar}_{1000}{ }^{+}\right)$in order to remove intact molecular ions while sputtering, to improve the qualities of molecular depth profiling and 3D imaging ${ }^{111}$. Work has shown that within the GCIB distribution, larger clusters such as $\mathrm{Ar}_{4000}{ }^{+}$lend themselves to be powerful analysis beams with significant SI yields, with capabilities exceeding $\mathrm{C}_{60}{ }^{+111}$. Ongoing work from various groups has shown promise in doping these large projectiles through interactions with reactive reagents such as $\mathrm{HCl}$ to increase SI yields ${ }^{112}$. These projectile doping efforts in combination well as surface modifications using reactive gas exposure to have shown to greatly enhance protonated ion yields ${ }^{113}$.

In 2006 Hiraoka and coworkers demonstrated the applicability of electrospray droplet impact ionization as a potential mechanism for massive cluster SIMS utilizing charged water droplets ${ }^{114}$. The initial version of this source employs 1M Acetic acid solution which are first generated in atmospheric conditions, and then introduced to vacuum conditions through a series of differential pumping apertures, and postaccelerated to the target plate with $\sim 10 \mathrm{kV}^{114}$. Like the MCI projectiles, these clusters have a vast amount of charges along with a significant mass component i.e. $\left[\mathrm{H}^{+}\left(\mathrm{H}_{2} \mathrm{O}\right)_{n}\right]$, with the average projectile being $\left[100 \mathrm{H}^{+}\left(\mathrm{H}_{2} \mathrm{O}\right)_{90000}\right]^{+100}$ or roughly $5 \times 10^{4} \mathrm{~m} / \mathrm{z}^{115}$. The ionization mechanism depends on the initial projectile generation energy, involves a combination of charge transfer via a solvation process or supersonic impact collision cascade $^{115}$. This research has also been exploring different mixtures such as 1propanol/water, which has shown promise in ionization of intact peptides ${ }^{116}$. These projectiles have been utilized to interrogate biological targets such as intact lipids and 
proteins ${ }^{117}$. They also have shown feasibility in depth profiling contexts, restricted primarily by the focusing potential of the ion column ${ }^{118}$. Notwithstanding, water clusters have also been generated utilizing GCIB-type designs. Work from the Vickerman lab has shown that water clusters could be generated in a nature similar to argon clusters by employing water vapor instead of argon gas ${ }^{119}$. $\mathrm{H}_{2} \mathrm{O}$ GCIB has been compared to $\mathrm{C}_{60}{ }^{+}$ and $\mathrm{Ar}_{2000}{ }^{+}$, providing enhanced intact molecular ion yields in comparison to both of these projectiles ${ }^{120}$.

In 2009, Fujiwara and coworkers performed preliminary work in evaluating the feasibility of ionic liquids as PI sources for SIMS ${ }^{121}$. They subsequently examined the effect of extractor electrode arrangements, vacuum interfacing, and solution temperature and solution flow rate on the current generated by these sources ${ }^{121}$. Their work concluded that ionic liquids are feasible PI sources, and continued to work on the subject ever since with a focus on generation of massive charged droplets for cluster SIMS. Current progress displays formation of drops with an $\mathrm{m} / \mathrm{z}$ from $1.5 \times 10^{5}$ to $2 \times 10^{6122,123}$. The pitfalls to this novel type of source is the gradual buildup of anion-cation layers when operating in a purely DC regime ${ }^{124,125}$. These layers accumulate due to electrochemical reactions at the interface area of the capillary tip, where a 'sandwich' of varying polarity ions form a solid residue, obstructing the flow of the capillary and reducing overall current output ${ }^{124-125}$. Unrelated work has proved that this rapid degradation of the capillary when spraying ionic liquids could be avoided by switching the polarity of the output at a rate of $\sim 1 \mathrm{~Hz}^{126}$. Fujiwara, however has not reported this type of particular degradation. Notwithstanding, in this early work the acceleration energy was generally a total of $+/-1 \mathrm{kV}$, only generating $\sim 20 \mathrm{nA}$ of current ${ }^{121}$.More recently, Fujiwara's group 
has been able to generate $\sim 100 \mathrm{nA}+$ ion streams utilizing a DC power source where a 30 $\mu \mathrm{m}$ stainless steel capillary is charged to generate the Taylor cone for the electrospray ${ }^{127,128}$. They report no degradation at low flow rates and low ionization voltage, yet this generally implies low source brightness and a preferential formation of smaller droplets ${ }^{129}$. They also have utilized the ionic liquids as matrix-coating materials (enhances ionization and yields) to match the projectile types ${ }^{129}$. 


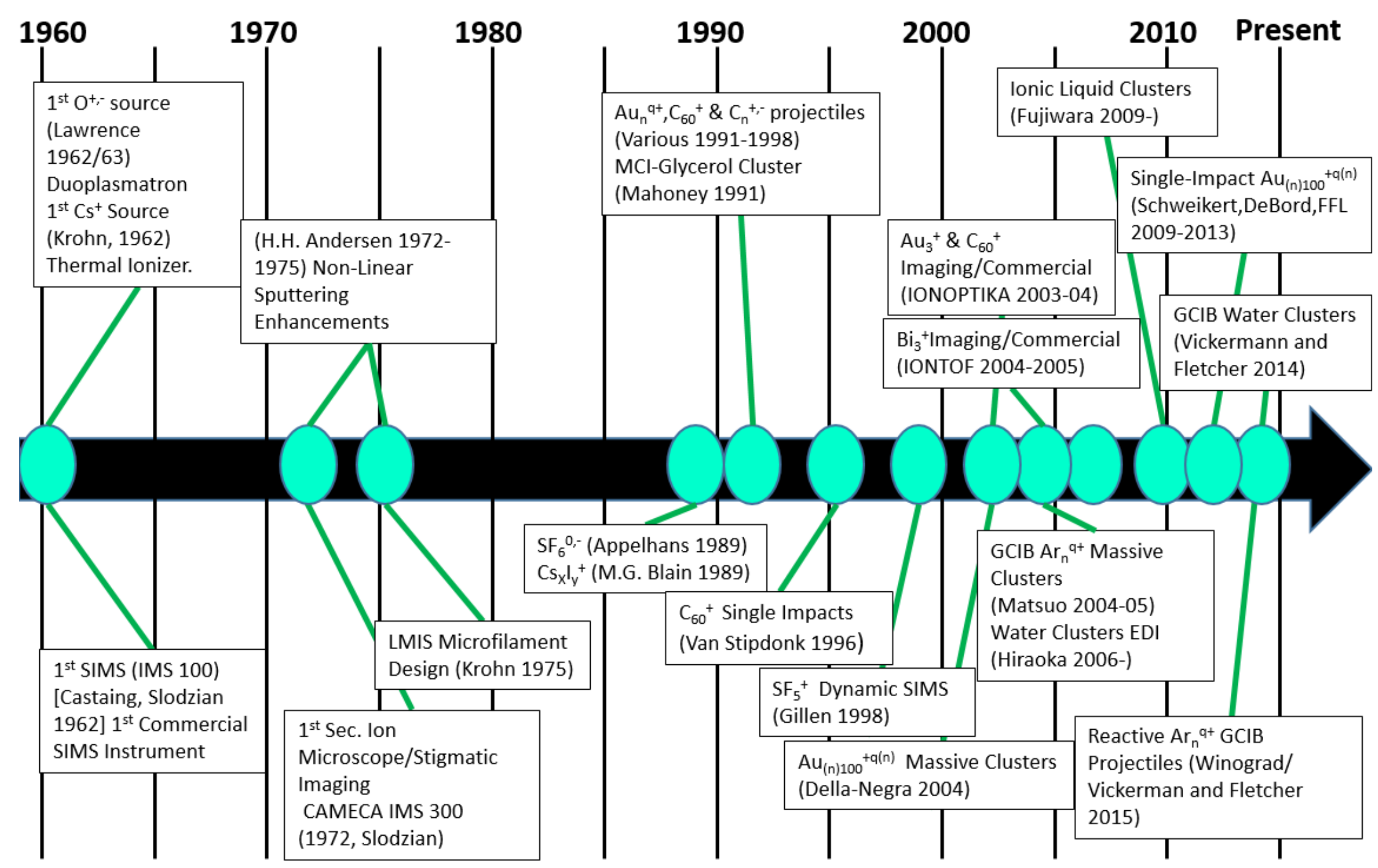

Figure 7. Timeline of the crucial developments in Secondary Ion Mass Spectrometry with an emphasis on the advancement of projectiles and operation modes. 


\section{DEVELOPMENT OF A MULTI-SOURCE PRIMARY ION COLUMN}

\subsection{Introduction}

The effectiveness of a focused ion beam is not limited only by source-specific features, but by the ion optics employed in the probe ${ }^{10}$. The optics contained within the column dictate the minimal possible spot size, or beam focus diameter. Optics also are employed to pulse, controlling the current/dose rate at which the sample is bombarded with, a critical feature in SIMS $^{10}$. Electromagnetic filters can also be employed to characterize the overall constituents of a PI source, and ensuring only one particular type of projectile irradiates the sample surface ${ }^{10}$. The overall focusing potential of a PI column relies on the capability of ion optics effectively correcting and reducing the aberrations that the PI beam is subject to ${ }^{11}$. The different types of sources mentioned in the introductory section have differing aberration effects and initial kinetic energies based on the PI generation mechanism ${ }^{11,56}$. This warrants a discussion of the components, their purpose, and the problems they seek to tackle in developing a high spatial resolution PI column for surface bombardment with cluster PIs.

\subsubsection{Ion Optics and Electrostatic Lens}

The main aberrations that affect focused ion beams are chromatic and spherical (geometric) aberrations ${ }^{130,131}$. Chromatic aberration is the kinetic energy spread between the ions, making the effective reduction of the beam-cross section difficult due to greater spread in ion energies when exposed to electrostatic or electromagnetic forces.

The chromatic aberration can be defined according to the focal length by the expression

$$
d_{c}=K_{c} r_{a} \Delta V / V_{0}
$$


where $K_{c}$ is a dimensionless factor with values $\mathrm{n}=1-5$ for einzel lenses, and $\Delta \mathrm{V} / \mathrm{V}_{0}=\delta$ being the relative kinetic energy spread of the particles ${ }^{130-131}$. Spherical aberrations revolve more around the geometry, or inherent spatial distribution of the generated ions and throughout their drift time. Severe spherical aberrations make focusing very difficult since the focusing optics possess a window of acceptance corresponding to the entrance angle of the incident ion, defined as $r_{a}$ and $r_{a} / 2^{130-131}$. These acceptance angles are themselves defined by the roundness and diameter of the aperture. The point in space at which the beam has the smallest cross-section is defined as the "circle of least confusion" and occurs generally at the end of the focal length, or the focal point. This minimum of the cross-section, or the place in space where the beam has the smallest beam diameter, is defined as the spherical aberration. This value can be obtained by the expression

$$
d_{c}=K_{a} r_{a}^{3} / D^{2}
$$

where $K_{c}$ is a unitless lens-specific constant value ranging $n=2-10$ depending on the operational mode of the lens. The maximum distance $r_{a}$ is defined by the roundness of the aperture, and $\mathrm{D}$ is a cm-scale scaling parameter. With these considerations, the core goal in developing a primary column is the implementation of optics which are capable of reducing these aberrations, thus allowing greater control over the beam cross-section at a desired focal length and the energy distribution of the ions ${ }^{130-131}$. Immersion lens, generally described as an axially symmetric array of 2 or more electrodes, are popular in this area because of their ability to reduce the overall cross section of the ion beam following an area of maximum dispersion or a disk of "most confusion"130-131,132. The "focal strength" of an immersion lens, measured as the ratio of the intra-lens field penetration to the focal length (D/f) is what dictates the refractive power of the lens, and 
subsequently the effective focal length ${ }^{130,132}$. Intra-lens field penetration itself is defined by the ratio of the internal diameter (D) of the electrodes and the distance between these electrodes (A). The implementation of an array of these lenses in tandem permits "zooming" of an image or ion beam, allowing for the reduction of millimeter crosssection beams down to the microscale and even nanoscale ${ }^{133}$.

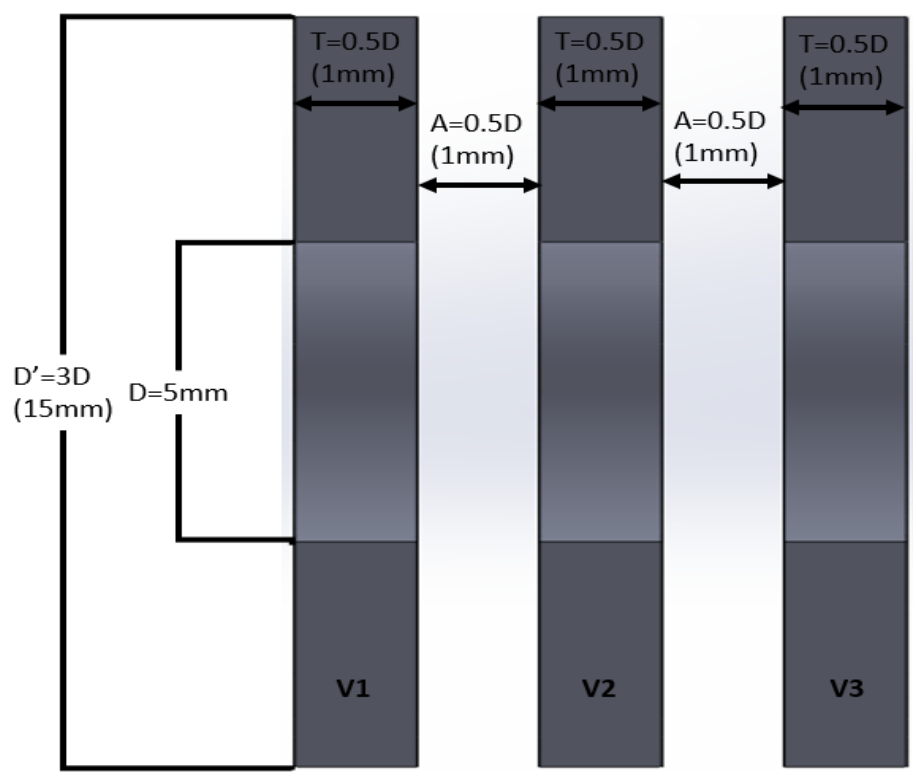

Figure 8. Scheme for a simple, symmetrical 3 electrode aperture lens. Relations between features dimensions demonstrated as a function of the aperture size, $D$.

Einzel lenses are the most common type of immersion lens, consisting of an array of three electrodes where $\mathrm{V}_{1}=\mathrm{V}_{3}=0$. The middle electrode $\left(\mathrm{V}_{2}\right)$ is the main determinant of the refractive index, making it the main electrode, and where the refractive effect is the same for incoming or outgoing beams in a field-free space. An important aspect of the symmetrical construction of these lenses is that the overall kinetic energy of the ions is not affected, but rather is homogenized, reducing chromatic aberrations $\left(\mathrm{eV}_{1}=\mathrm{eV}_{3}\right)^{130,132}$. Construction practices vary on the focal length, geometrical arrangement constraints, and electronics arrangement within the PI column ${ }^{130,132}$. 


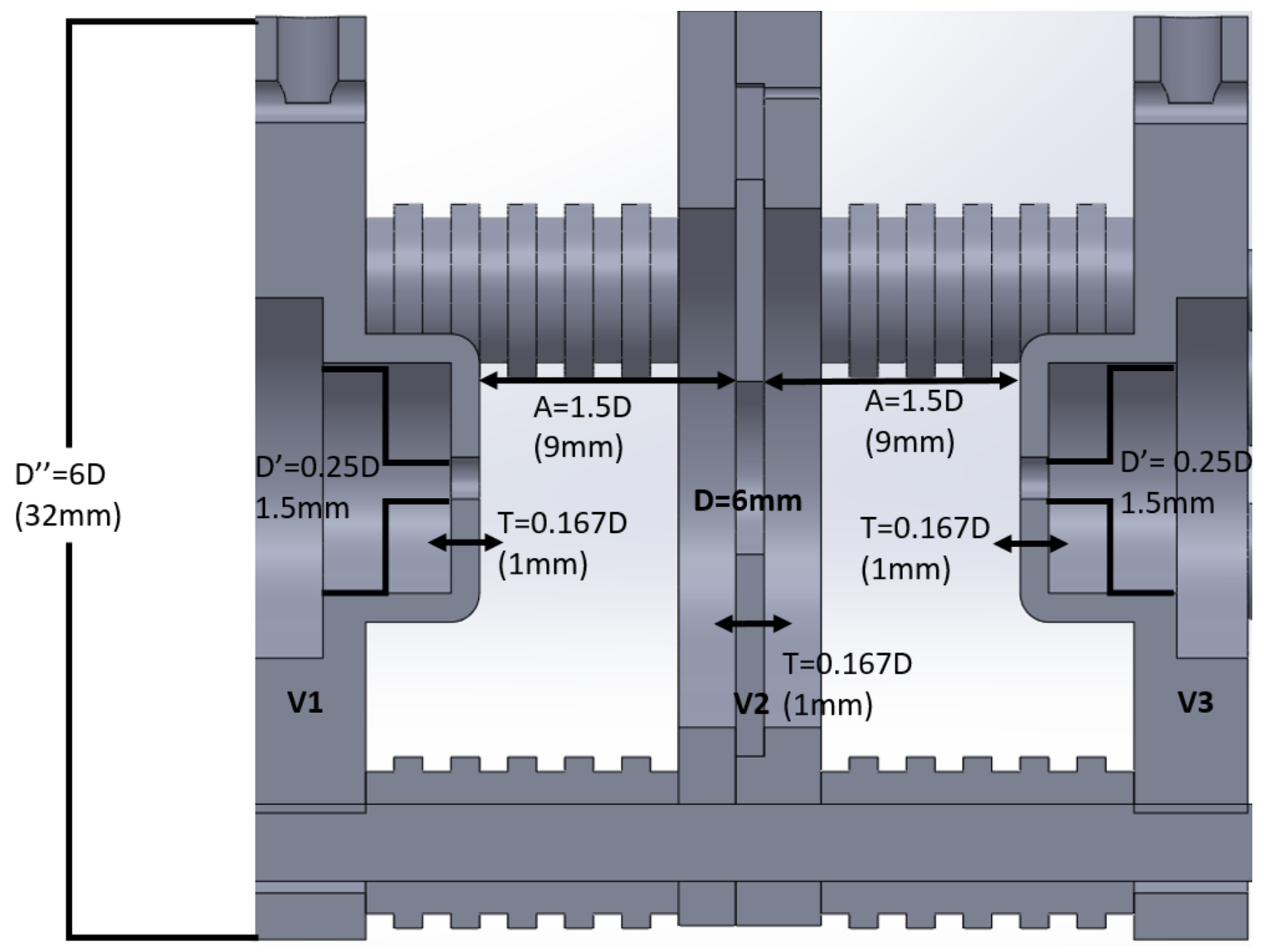

Figure 9. Scheme for a deacceleration-acceleration aperture einzel lens $(\mathrm{V} 1=\mathrm{V} 3=0)$. Note the varying aperture sizes and their respective ratios, as these influence focal length and focal strength $(\mathrm{D} / \mathrm{f})$.

The most common einzel lens construction can be operated as a three discrete element system, where $V_{2}$ is comparable in magnitude to the source voltage. Such an arrangement generates a deaccelerating-accelerating effect with a significant amount of refractive power for attenuating chromatic and spherical aberrations. These types of lenses generally are limited to short focal lengths, but are very easy to implement and can be operated in tandem to achieve serial focusing ${ }^{130,132,133}$. 


\subsubsection{Primary Ion Filter: Wien Filter}

Once a desirable level of focusing is achieved, the major concern becomes the ability of characterizing the generated projectiles, as well as filtering the PI beam. It has long been reported that most cluster projectile PI columns require a discrimination filter, allowing for only one specific $m / z$ projectile to impact the target surface ${ }^{11}$. Most of these instruments tend to be equipped with electromagnetic filters, such as sector-type discriminators and Lorentz-force driven mass filters. For the sake of keeping aberrations to a minimum, linear-type separators, such as Wien Filters have become the research and industry standard for PI beam constituent separation ${ }^{134}$. The Wien filter is an ion velocity filter, used to eliminate all undesired projectiles. The trajectory of the ion upon entering the Wien filter is based on the aggregates of the electrostatic and magnetic forces perpendicular to each other, creating a net Lorentz force also influenced by coulombic force, leading to a net force based on the expression ${ }^{131,134}$ :

$$
F_{\text {net }}=F_{E}+F_{L}=(q \vec{E})+\left(q v_{\text {ion }} x \vec{B}\right)
$$

The filtering effect, as mentioned above, is dependent in the net velocity of the ion and with respect to the net aggregation of forces in the filter. Transmitted ions corresponding to a certain mass to charge ratio where the net force is zero ${ }^{131,134}$.

$$
\begin{gathered}
0=(q \vec{E})+\left(q v_{i o n} x \vec{B}\right) \\
v_{0}=\left(\frac{\vec{E}}{\vec{B}}\right)
\end{gathered}
$$


Based on the prior equation, a substitution for vion can be used to alter the equation in order to calculate the voltage needed for projectile permissibility

$$
v_{i o n}=\sqrt{\frac{2 K_{e}}{m_{i o n}}}
$$

By substituting the superimposition of forces by the velocity the resultant expression becomes ${ }^{131,134}$

$$
\sqrt{\frac{2 K_{e}}{m_{\text {ion }}}}=\left(\frac{\vec{E}}{\vec{B}}\right)
$$

From here, expansion of the force expressions for the coulombic force, $E$, allows for the correlation of a specific voltage with respect to a fixed magnetic field, $B$. Other variables of importance are the distance between the electrostatic plates, $d$ and the incoming energy, $K_{\mathrm{e}}$, of the PI as well as its mass $\left(m_{i o n}\right)$. In order to determine the voltage for transmission, the electrical force $\mathrm{E}$, is changed to its constituents ${ }^{131,134}$

$$
E=\frac{V}{d}
$$

Considerations have to be taken for the the plates being equipotential but of opposite polarity, $\mathrm{V} \pm$

$$
V=B d \sqrt{\frac{2 K_{e}}{m_{i o n}}}
$$




$$
2 V_{ \pm}=B d \sqrt{\frac{2 K_{e}}{m_{i o n}}}
$$

By rearranging the expression to account for these differing potentials, the final expression for determining the transmission voltage becomes

$$
V_{ \pm}=B d \sqrt{\frac{K_{e}}{2 m_{\text {ion }}}}
$$

\subsubsection{Collimation and Electrostatic Deflectors: Pulsing}

Other additional elements in PI columns generally revolve around beam collimation, pulsing, and deflection in order to ensure pure concentricity throughout the arrangement of optics. In optics, sometimes mechanical collimation, or "trimming" of a beam is required in order to reduce the proliferation of aberrations, by ensuring that only ions within a specific trajectory are transmitted ${ }^{135}$. This generally reduces the brightness of a source, with the goal of increasing the resolution of the image object, in this particular case by reducing the beam diameter of the incident PI beam. Collimation is widely utilized in high-spatial resolution PI columns as the trajectories of ions are increasingly erratic depending in the kinetic energy distribution of ions at the source point. However, if the spatial trajectory distribution of the PI beam is too severe and the brightness of the source is low, collimation may prevent the passage of a stable beam of desired projectiles $^{135}$. An important factor that was mentioned in the introduction of this thesis is the importance of the pulsing of a PI beam in order to mass select projectiles and control 
PI beam dosage. As described in Chapter 1, electrostatic deflection plates are a highly effective approach to select ions and control bombardment. By programming two of these to work in tandem by coordinated start-stop signaling, specific PIs can be mass-selected based on their time of flight ${ }^{135}$. In essence, this equips a PI column with an internal TOF mass spectrometer as a means of selecting particular projectiles. In controlling the rate pulses are applied, the amount of ions that impact the surface can be controlled. Time of flight $(\mathrm{t})$ can be calculated from initial kinetic energy input $\left(\mathrm{K}_{\mathrm{e}}\right)$. With this initial energy input, ions will travel through the flight path distance (d) with a velocity corresponding to their mass $(\mathrm{m})$. The derivation for time of flight is described below ${ }^{131}$ :

$$
K_{e}=\frac{1}{2} m v^{2}
$$

Which can be arranged to resolve for the velocity of the ion

$$
v=\sqrt{2 K_{e} / m}
$$

However we also know that velocity is displacement over time

$$
v=\frac{d}{t}
$$

By combining these two definitions of velocity, an expression that allows us to solve for the mass based on energy and flight time over a fixed distance is obtained. These variables are condensed as the factor A since they're fixed (length of the tube and initial potential pulse). 


$$
m=\left(\frac{2 E}{d^{2}}\right) t^{2} ; A=\left(\frac{2 E}{d^{2}}\right)
$$

Replacing the expanded expression with A yields:

$$
m=a t^{2}
$$

However, given that TOF analyzers are not continuous in nature and have a pulse down-time, this must be substracted from the total time to provide a corrected time $\left(t_{c}\right)$, substracting the pulse down time from the measured time $\left(t_{m}\right)$.

$$
t_{c}=\left(t_{m}-t_{o}\right)^{2}
$$

Combining these two expressions yields a final expression for which mass can be calculated based on the corrected time of flight.

$$
m=A\left(t_{m}-t_{o}\right)^{2}
$$

This information can then be set to control the frequency of the pulsing for the PI beam. It must be noted that this also affects the overall duty cycle of the instrument, as mass selecting projectiles which are less abundant within their particular distributions has been noted to increase analysis times. 


\subsection{Methodology}

Developing instrumentation involves consisting of planning and preliminary design. This is then followed by the deployment of theoretical tools to explore the performance of the proposed design, in a cost-effective and highly accurate form. Upon the satisfaction of given criteria for the instrument, such as specific beam diameter for a PI probe, one can then commence the processes of fabrication. However, there may always be discrepancies between the simulations and experimental performance of the instrument, so this process can also be used to perform "reverse-engineering" in order to test defects in the preliminary design.

The development of this PI column for SIMS followed this process, with the main challenge being the design of optics capable of reducing PI probe diameters to $10 \mu \mathrm{m}$ or less for microscale analysis. This type of beam diameter, as aforementioned, is analogous to the spatial resolution capabilities of the PI column. Additionally, a Wien filter is employed to characterize the specific PIs found within the probe, as specific ion impact. A set of collimating apertures were also added to eliminate ions with erratic trajectories, varying in size. Two sets of deflection plates are placed to ensure PI beam alignment. Additionally, it is important to experimentally determine whether it is better to pulse the probe immediately following separation inside the Wien Filter or shortly after the final focusing optics. This is crucial in static SIMS operation as the placement of the pulsing affects the overall spatial resolution. 


\subsubsection{Design (Solidworks/CAD) and Fabrication}

The design stage employs computer assisted design (CAD) software, such as Solidworks 2013 (Dassault Systemse; Velizy-Villacoublay France). Components are designed individually, around a common housing structure to generate an assembly. Assemblies are then united to create an overall structure. This overall structure is set to certain specific machining standards for precision purposes. Taking advantage of modern advances in machining, components designed by CAD software can be fabricated based on the spatial parameters and features present on the part. Meaning, a part can go directly from a virtual design to a physical piece with little difficulty. Tools such as CNC mills and 3D printers allow for the fast prototyping of parts if no theoretical tools are present to address performance testing. CAD software also allows for the seamless transfer of the 3D structure of a part or assembly to be transferable to theoretical tools such as simulation programs. Even though these programs come with integrated design tools, it is very difficult to capture the nuances of very elaborate component designs.

\subsubsection{SIMION Simulations for Value Optimization}

Upon finalizing the design stage for the development of the PI column, it is possible to evaluate the effectiveness of the ion optics with dynamic ion simulations. Features such as geometrical arrangement, PI generation conditions, and optimal operation value such as voltages and magnetic fields can be optimized this way ${ }^{136,137}$. In evaluating ion optics, very few tools have become as indispensable as the ion dynamics simulation software SIMION. SIMION allows for the visualization of ion behavior when exposed to electrostatic and electromagnetic forces generated from physical arrays ${ }^{137}$. 
Ions are given initial generation features such as kinetic energy, spatial distribution arrangements, directional vectors, mass to charge ratios, and charge related ion repulsion.

The first step in evaluating the effectiveness of the components is to import the physical objects such as electrodes, with their spatial arrangement remaining as true as possible to the original design. This can be achieved by utilizing the physical array drawing tool in SIMION or by converting the Solidworks assembly into an ASCII format via tessellation of object layers as triangles. This generates what is known as a Standard Tessellation Language file, or a .STL, which can be transferred into SIMION work with electrostatic/magnetic properties. Once all components from the design have been transferred and geometrically arranged, simulations can be performed. SIMION permits the user to record a variety of different information regarding kinetic energy, trajectory, spatial distribution, and electromagnetic forces experienced by the ions ${ }^{137}$. These can be recorded at the start, during the drift time, at a specific location in space, or as a function of each ion time-specific step at a given rate. It's contributions to MS development throughout the years are significant ${ }^{138,139,137}$. 


\subsection{Results and Discussion}

\subsubsection{Solidworks (CAD) designs of Primary Ion Column Components}

The PI components were optimized based on theoretical ion dynamic simulations, expected performance and floor-plan considerations. Some of these considerations involve the prevention of electrostatic discharging, and the retention of high-vacuum $\left(10^{-}\right.$ ${ }^{6}$ torr $\left.\geq\right)$. The source and einzel lens housing was the first component to be drafted. It was designed to be equipped with 6 in Con-Flat (CF600) flange mountings present for the PI source mounting stage on the front, and pre-filter einzel lens (Figure 10) in the rear, leading to the Wien Filter (Figure 11). Additionally, there are mountings for a quartz CF600 side viewport (not pictured), a turbo-molecular vacuum pump, and a CF600 flange with SHV feedthroughs.

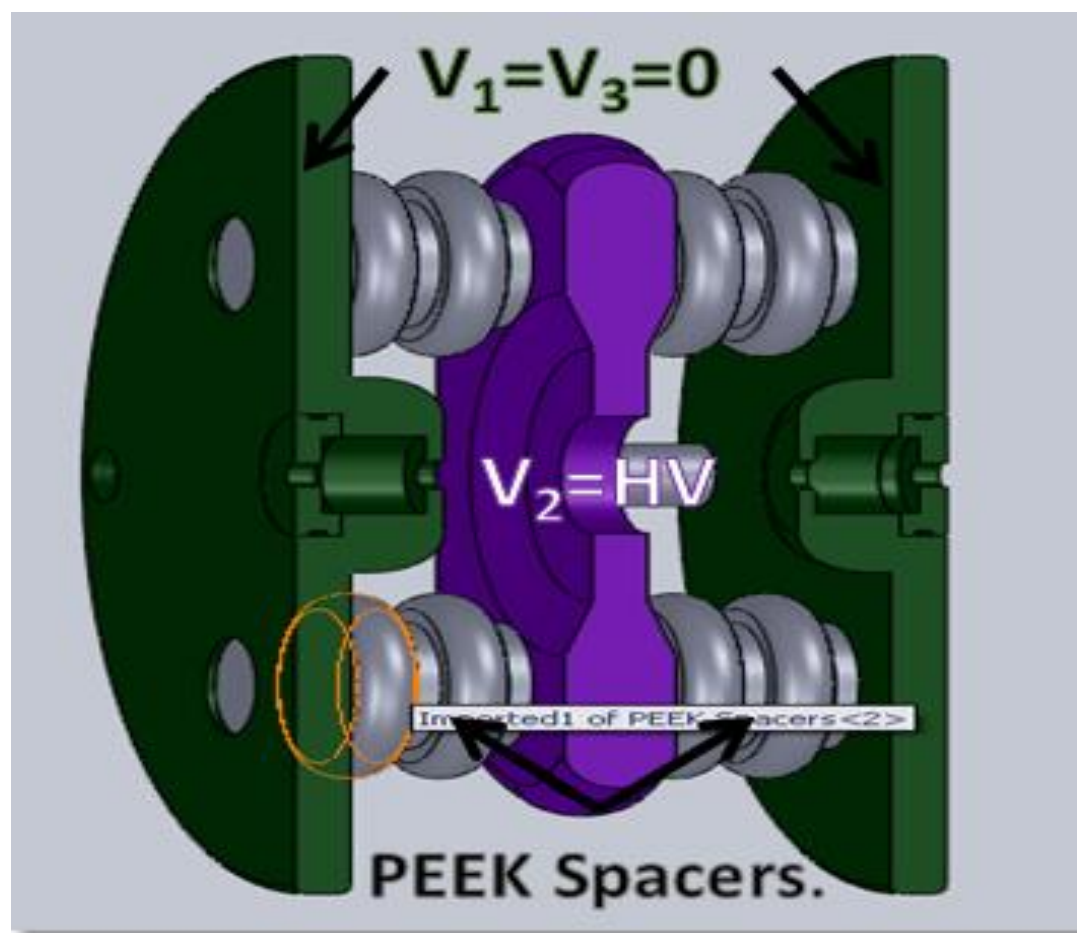

Figure 10. 3D cutaway view of the design for the pre-filter einzel lens with potential values and key structural features highlighted. 


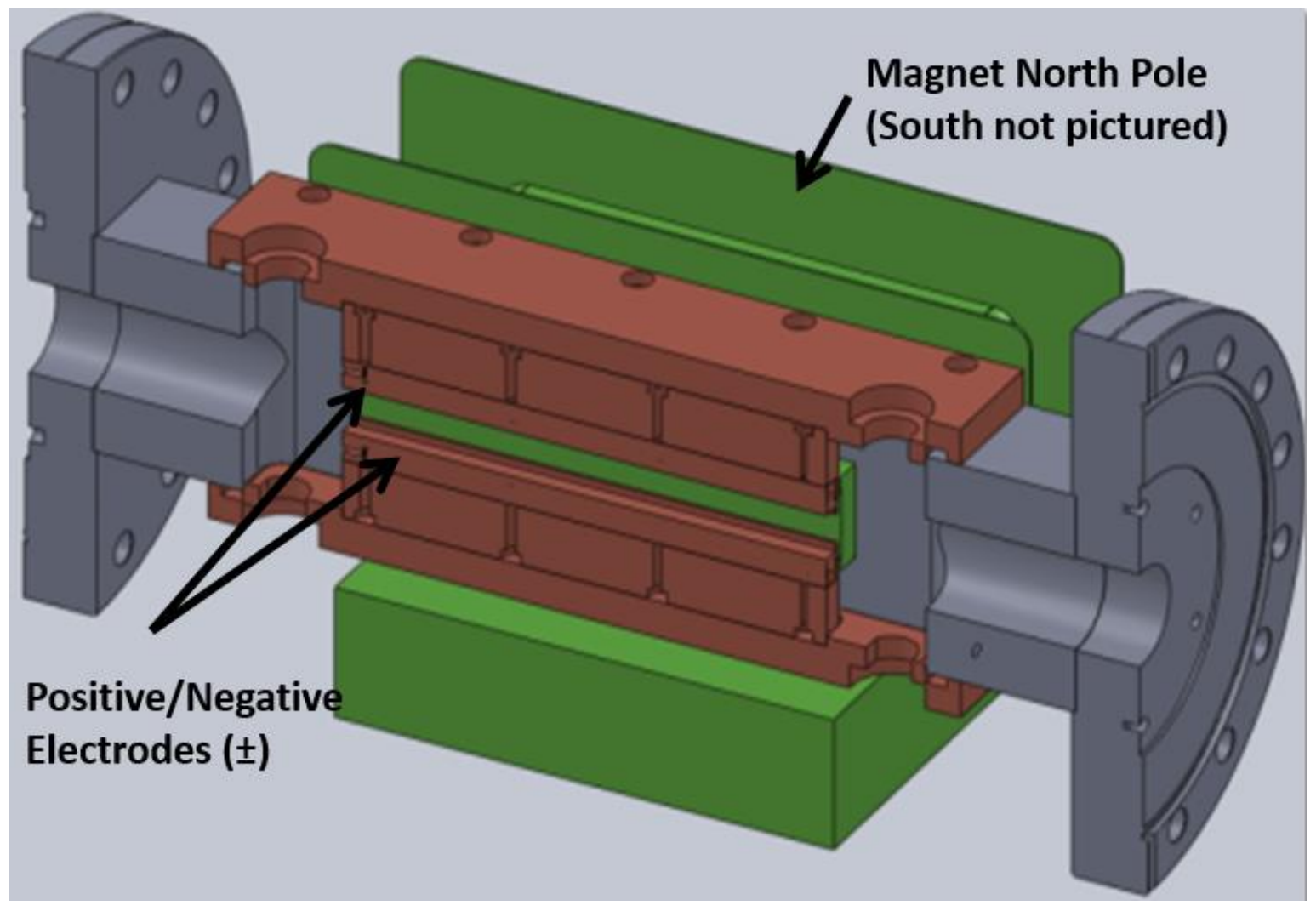

\section{Figure 11. 3D cutaway view of the design for the Wien Filter with key components highlighted.}

The source stage is based on the criteria of $\mathrm{X} / \mathrm{Y}$ control over the source positioning at submillimeter resolution. The stage picked for this was a Thermionics EC-XY B275C capable of supporting 2.75 in Con-Flat (CF275) flange-mounted sources. Stage resolution is important when precisely aligning concentric to the other optics, as any drift in this area will lead to odd ion entrance angles and unwanted surface charging effects. Ease of reproducibility when aligning is also critical when changing out sources due to wear. Projected plans for this mounting involved the usage of any LMIS as well as other PI sources. Pre-filter einzel lens are located at $\sim 90 \mathrm{~mm}$ from the source mounting, where three $86 \mathrm{~mm}^{3}$ outer diameter rounded slit electrodes are connected by insulating standoffs. The aperture configuration matches that which was presented earlier for 
deaccel-accel lens $\left(D_{1}=D_{3}<D_{2}\right)$, where $D_{1}$ and $D_{3}$ are $3 \mathrm{~mm}^{2}$ and $D_{2}$ is $12 \mathrm{~mm}$, a ratio of $\mathrm{D}=0.25 \mathrm{G}$.

The einzel lens is attached to the exit part of the housing, linking the source chamber to the Wien Filter. This is designed in such a manner that the projected focal point is at the collimation slits, beyond the Wien Filter. Design of the Wien filter was based on the original IPNO design, with the only difference being the ability to modify the electrodes rather than a permanent weldment ${ }^{99}$. The housing is meant to support electrical inputs, as well as structurally support the solenoids, their housing and the ferromagnetic steel required to create the magnetic field. The electrodes in the filter have a length of 123.6 $\mathrm{mm}$ and a spacing of $12.5 \mathrm{~mm}$, respectively. The mounting for the solenoids (electromagnet) was designed to be made with Teflon, and with the electromagnet cores made with roughly 60 turns of 16-gauge copper wire on each core.

Following the Wien Filter chamber, comes the diagnostic chamber where there is a set of optics for evaluating PI beam performance. There is a set of XY electrostatic deflectors for ensuring beam alignment prior to collimation and as a potential pulsing site (Figure 13). These have dimensions of $25.4 \mathrm{~mm}^{2}$, with $6.2 \mathrm{~mm}$ spacing between. At a distance of roughly $90 \mathrm{~mm}$ from the plates, there is a modular collimator array, with sizes spanning from $2 \mathrm{~mm}, 1 \mathrm{~mm}, 500 \mu \mathrm{m}$, and $200 \mu \mathrm{m}$ (Figure 14). The appropriate collimator size can be selected by an $\mathrm{X}$-axis CF275 rotary motion feedthrough which is controlled externally (MDC Vacuum). This chamber is also equipped with six CF 2.75 mounting slots, 2 axially positioned and 4 laterally positioned, respectively. 


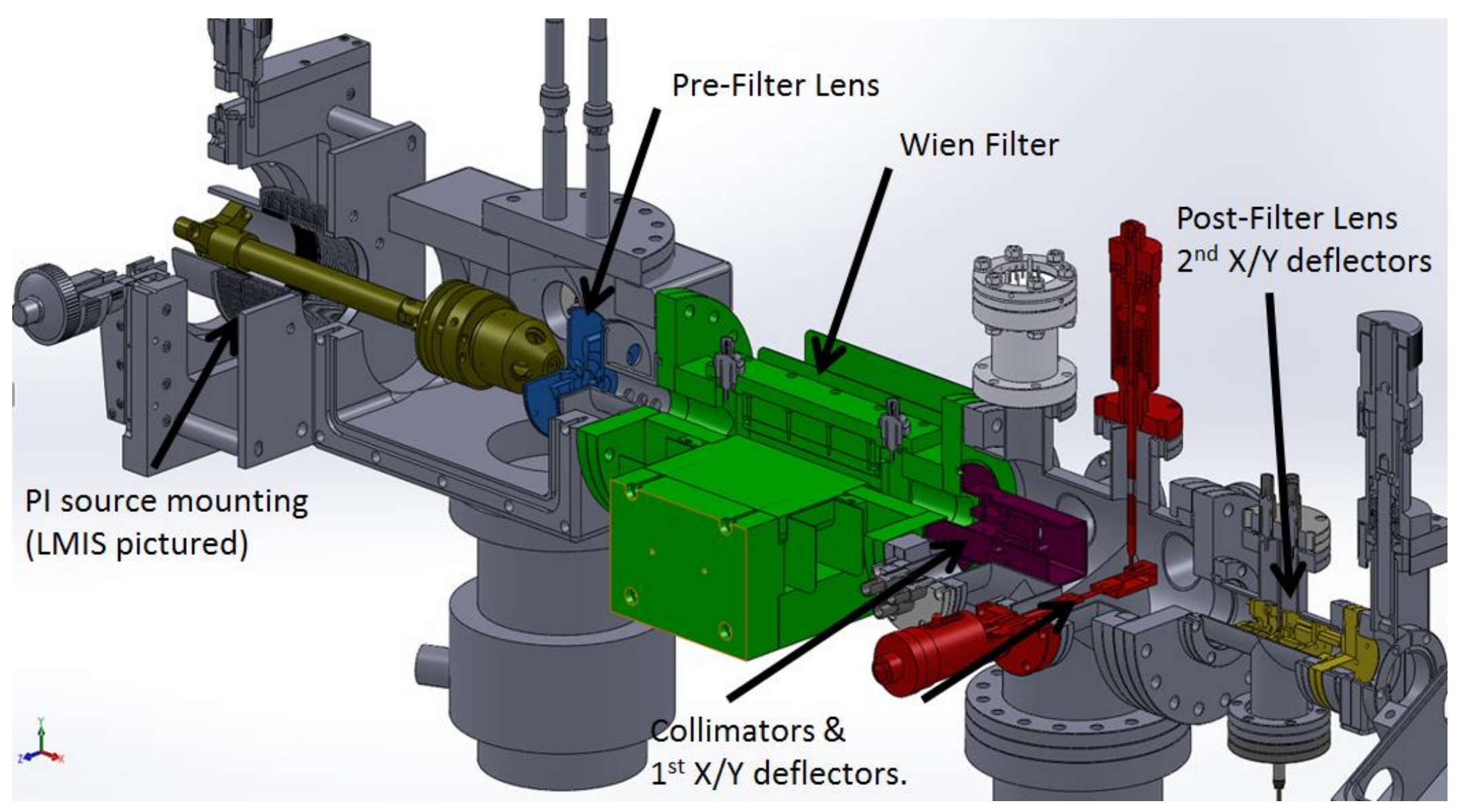

Figure 12. 3D cutaway view of the design for the primary ion column with key components for beam modification highlighted. 


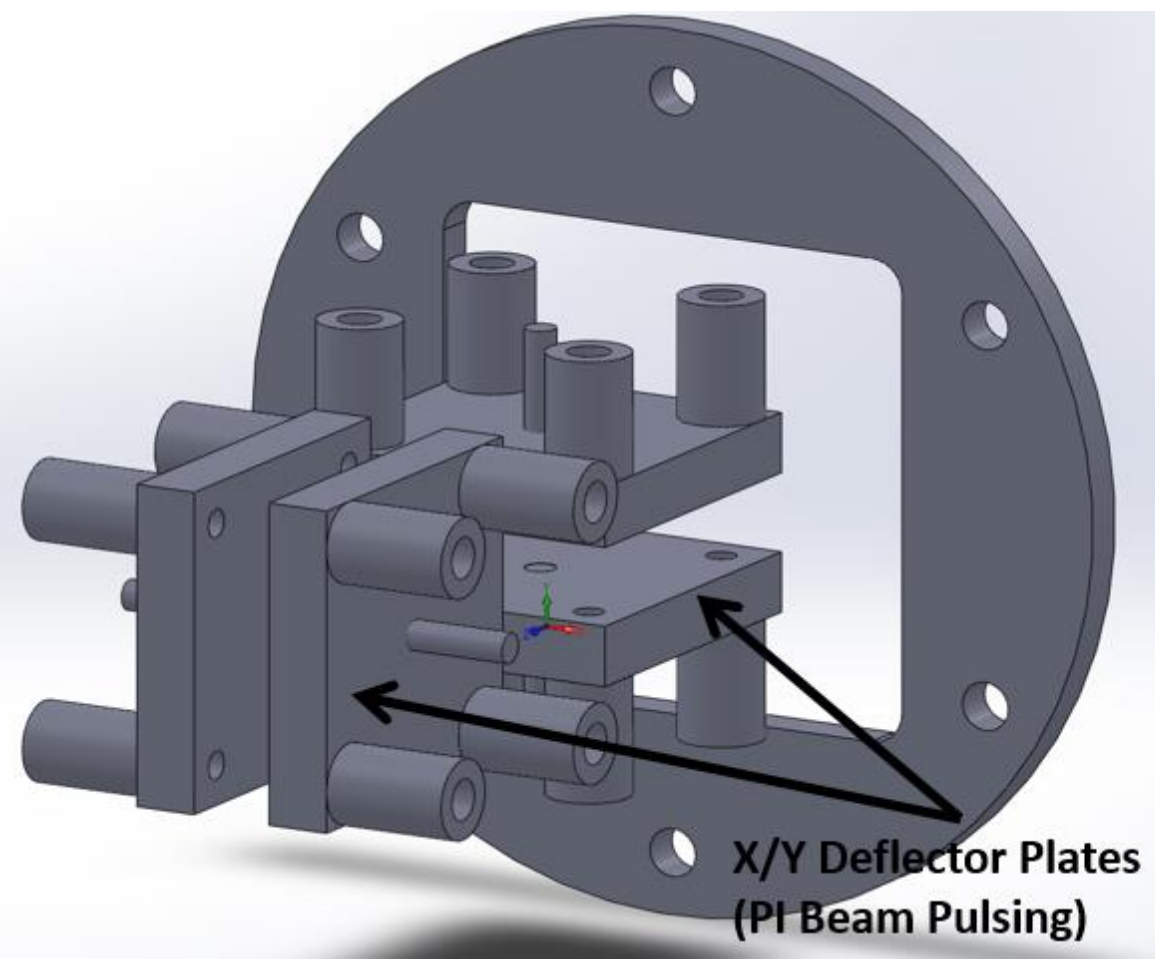

Figure 13. 3D view of the first set of electrostatic deflection plates for beam pulsing and alignment with key components highlighted.

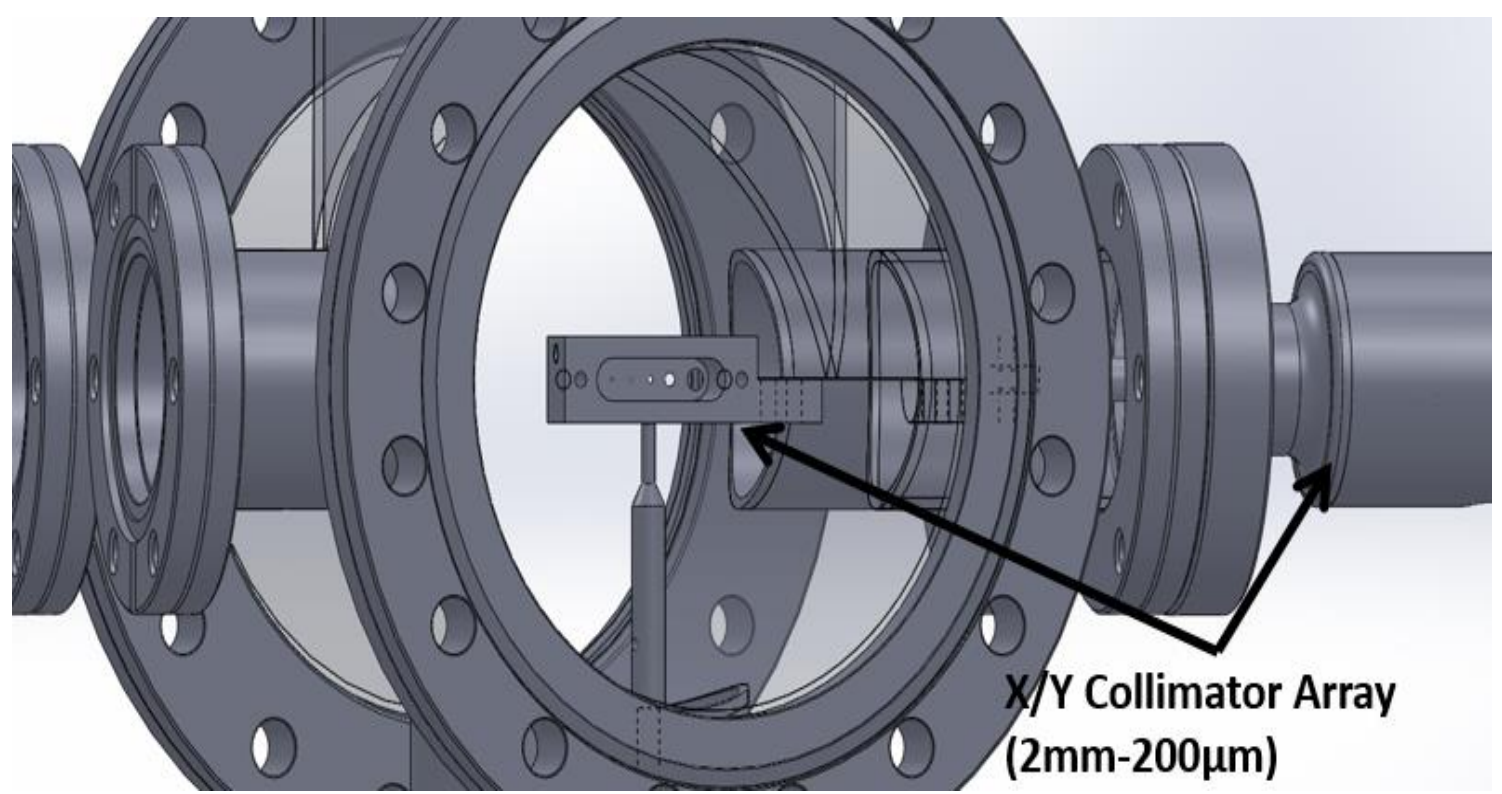

Figure 14. 3D cutaway view of the XY-controllable beam collimation array with key components highlighted. 
At the exit of the chamber, there is a mount for a CF600 to CF275 modifier, where a nipple extension can be placed. In such an extension, it was found beneficial to place a set of post-filter einzel lens to focus the centered, collimated, single-projectile PI beam prior to sample ionization (Figure 15). These lens were made to have identical apertures $(D=0.25 G)$, yet the outer diameter of the electrodes is $25.5 \mathrm{~mm}$ to fit inside the

nipple extension. The electrodes are mounted on insulating poles, separated by insulators with roughly $11 \mathrm{~mm}$ of spacing and with the second electrode shielded by an insulating enclosure in order to avoid discharging. In addition to the lens, a secondary set of XY deflector plates with dimensions of $20 \mathrm{~mm}$ x $10 \mathrm{~mm}$ is located at the exit in order to ensure beam concentricity prior to impact and as a potential pulsing site. The overall length of the structure is $\sim 55 \mathrm{~mm}$, allowing it to fit within a CF275 nipple flange. Other important features that bear mentioning involve the placement of a CF275 vacuum gate valve at the exit of the PI column. From here the instrument can be interfaced to a variety of chambers for surface modification studies and for MS analysis (e.g., TOF or FT-ICR MS).

\section{Vacuum components}

Aside from the main electrostatic and electromagnetic components, other important considerations involve the usage of vacuum equipment, as a PI column requires pressure on the order of $>10^{-6}$ torr to be effective. This is achieved by deploying an array of roughing pumps and two turbo molecular pumps located at the source and mid-focal distance, respectively. A combination of two pirani gauges (10-2 torr) and two BayardAlpert gauges $\left(<10^{-6}\right.$ torr $)$ is used to measure the effectiveness of the vacuum. 


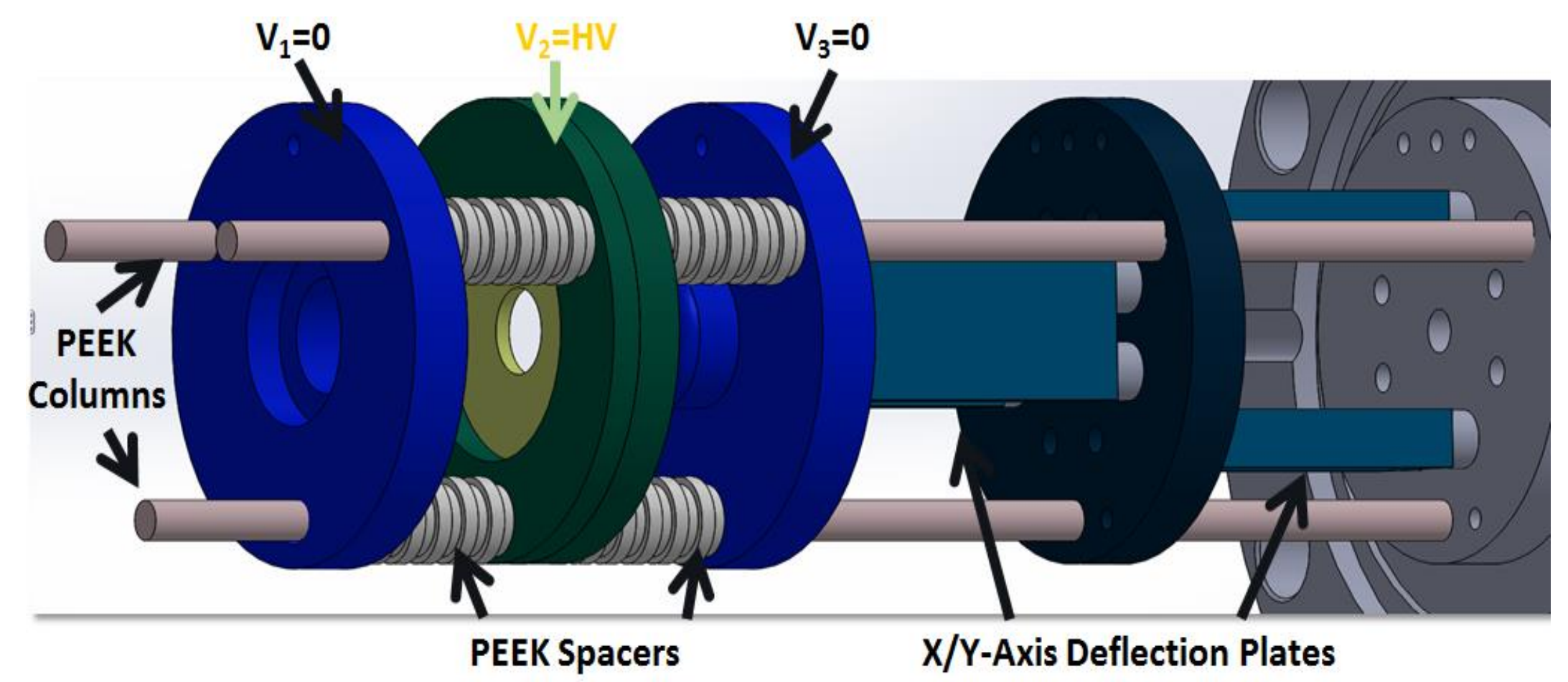

Figure 15. 3D view of the post-filter einzel lens and secondary set of electrostatic deflection plates with key components highlighted. 


\subsubsection{SIMION Array of Primary Ion Column Components}

The simulation of the PI column optics was performed in a series of steps. First, the Solidworks designs for the lenses, filter and collimating optics were transferred to a .STL file format. These were all scaled in a rating of 0.1 pixels $/ \mathrm{mm}$, such that the resolution and realism of the simulated electrostatic/electromagnetic fields was highly accurate. The Laplace transforms SIMION employs in predicting ion trajectory/dynamics is influenced by this scaling factor, so the accuracy of spatially relevant information is affected. Thus, depending on the size and number of features in the ion optics work bench (IOB), a certain resolution is required in order to confidently propose theoretical values or results drawn from simulations. Having made STL files with such a scaling factor, these were then modified into Physical Array (.pa\#) files, which account for the physical shape of the electrode/magnet. The arrangement of the different physical arrays was done with respect to the dimensions and spacing of componenets inside the PI gun from Solidworks.

Arrays with field-overlaps are also "woven", or that having certain common electrodes allows for the unison of different physical arrays as one unit. This affects simulation accuracy, with fields being improperly simulated, producing in null results. Upon completion of arranging the .pa\# files, simulations can take place by defining the SI properties. For the purpose of these simulations, three beams corresponding to $\mathrm{Au}^{+}, \mathrm{Au}_{3}{ }^{+}$, and $\mathrm{Au}_{400}{ }^{+4}$ were used. The general goal was to perform a qualitative evaluation of each component and their performance. The collection of operational values will vary from source to source, depending on the initial PI kinetic energy and spatial distribution. 
The first einzel lens displayed the intended performance, with focusing of the beam becoming evident with increasing voltage as a product of a deacceleration/acceleration mechanism inherent to the geometry (Figure 16).

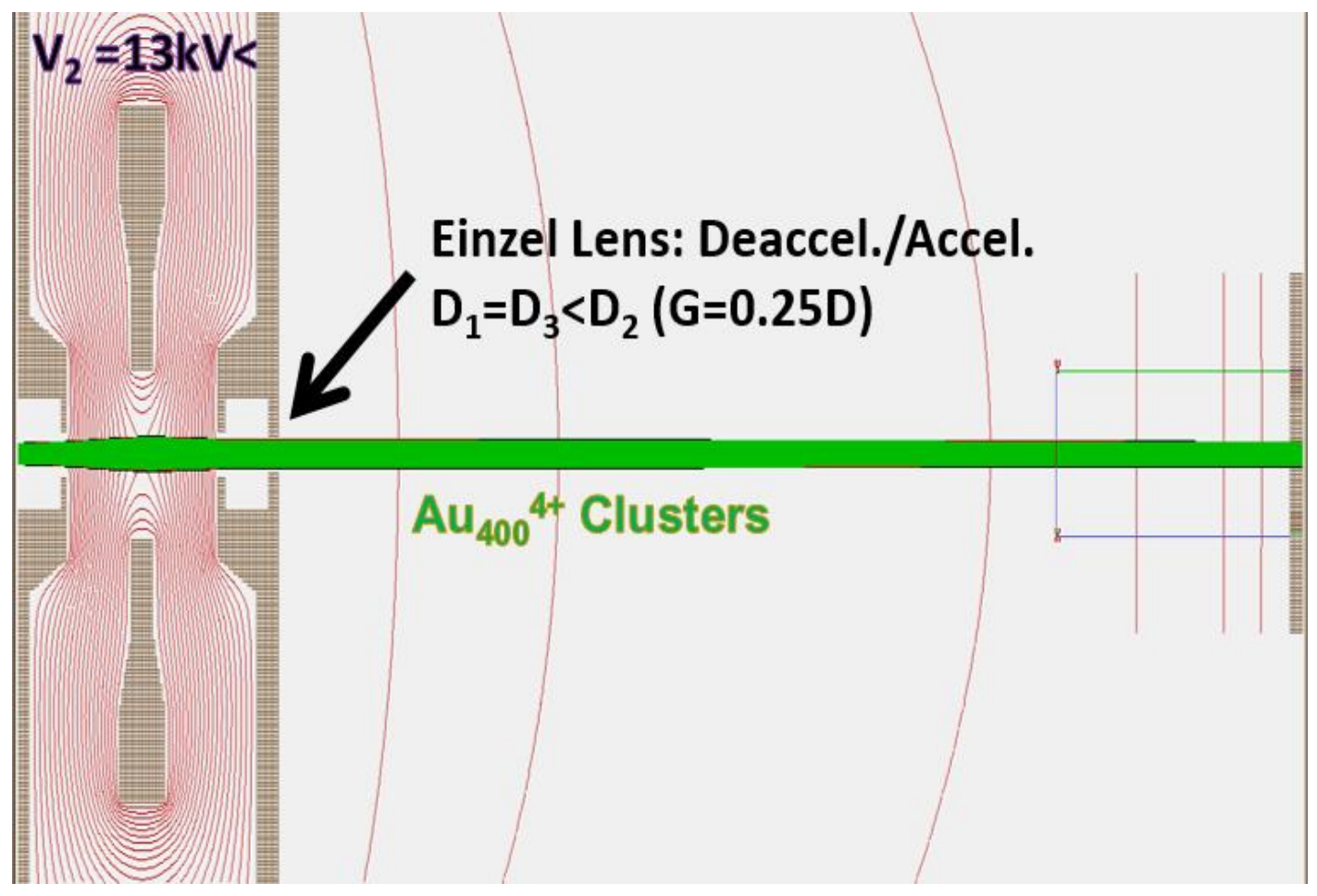

Figure 16. Simulation view of the pre filter-einzel lens performance with optic geometry features highlighted.

Peformance was tested in the $12.5-16 \mathrm{kV}$ range, providing an increasingly narrower focal length with higher voltages without full coverage of the PI column ( 60\%). This indicated that the design of a second lens, placed at a distance of $150 \%$ of the focal length $(1.5$ 'D/f"), at a scaled sizing of $0.5 \mathrm{G}(1: 2)$, could allow the elongation of the focal point for the full length $(1173.2 \mathrm{~mm})$ of the column. This lens also further reduces the cross section at the disk of least confusion, with a beam diameter (spot size) of $10 \mu \mathrm{m}$ or less being a reasonable expectation. 
The following aspect was to evaluate the performance of the Wien Filter. The magnet coils were set up to generate a magnetic field strength of $\mathrm{B}=0.175 \mathrm{~T}$, and the electrodes were set to $\mathrm{V}=15 \mathrm{~V}( \pm 7.496 \mathrm{~V})$, based on the expression derived above. This magnetic field value is based on theoretical calculations for such a design.

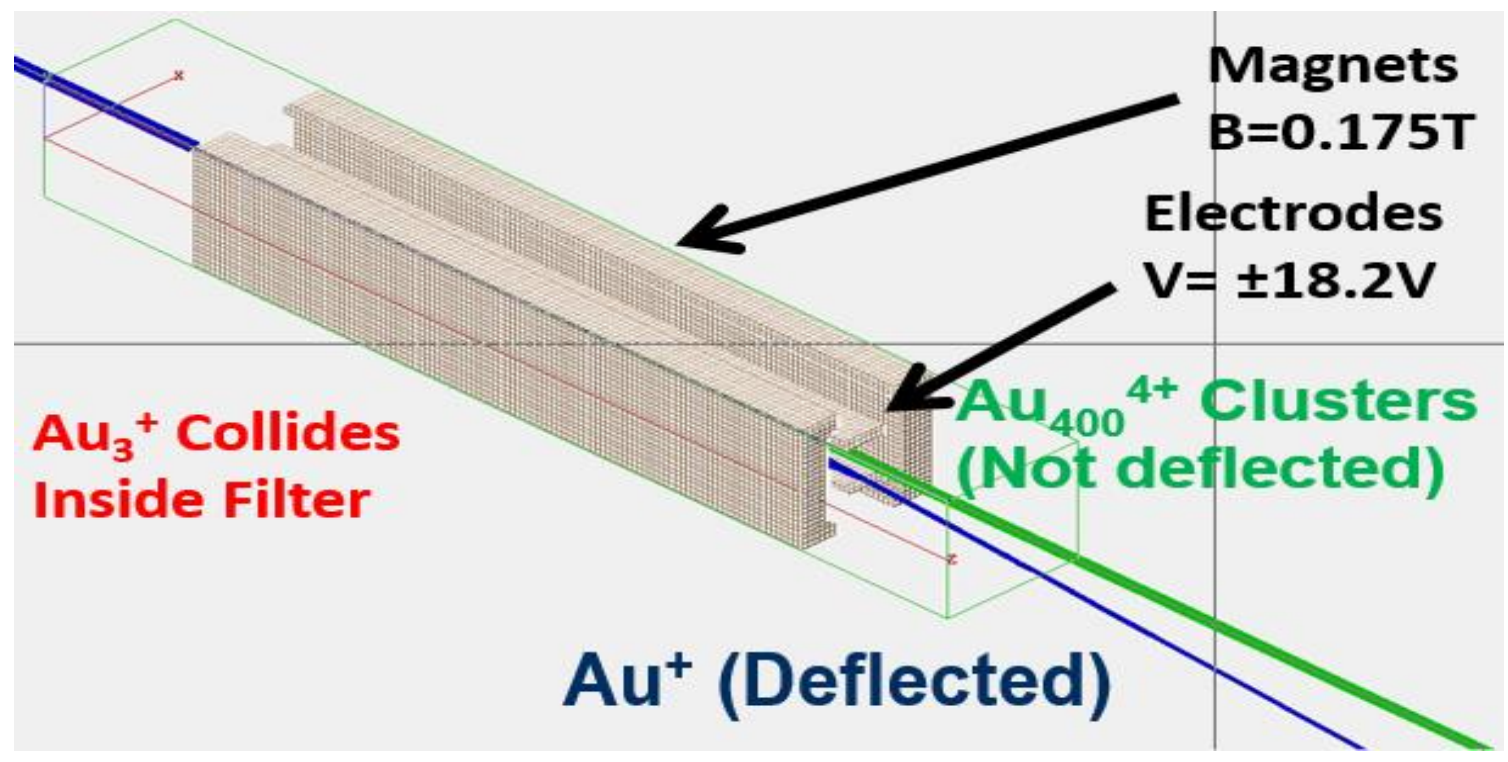

Figure 17. 3D view of Wien Filter performance simulation for selection of massive gold clusters with components highlighted.

$$
\begin{gathered}
V_{A u_{100}^{+} \pm} \pm B(T) d(m) \sqrt{\frac{K(J)}{2 m(a m u)}} \\
=(0.175 T, \text { Theoretical })(0.0123 m) \sqrt{\frac{3.204353 \times 10^{-15} J}{2 *(196966.569 a m u)}} \\
V_{A u_{100}^{+} \pm=15 V(\text { Theoretical })}
\end{gathered}
$$

The Wien Filter response as a function of the plate deflection volatges permitted the separation of the Au cluster ions (Figure 17). Collimating slits were added, with the 
standard $200 \mu \mathrm{m}$ ID aperture selected. Following collimation, the beam advances to the second electrostatic lens and XY deflectors. The $2^{\text {nd }}$ lens were use to prolong the focal length of the beam to any desired point in the remaining length of the PI source with an operational range of 3.5-8 kV (Figure 18). Like the first lens, the operational trade off involving focal length and beam diameter are dependant on the PI source inherent spatial distribution properties. A potential energy surface array simulation (Figure 19) was also performed with all the componenets to evaluate the electrostatic fields and forces.

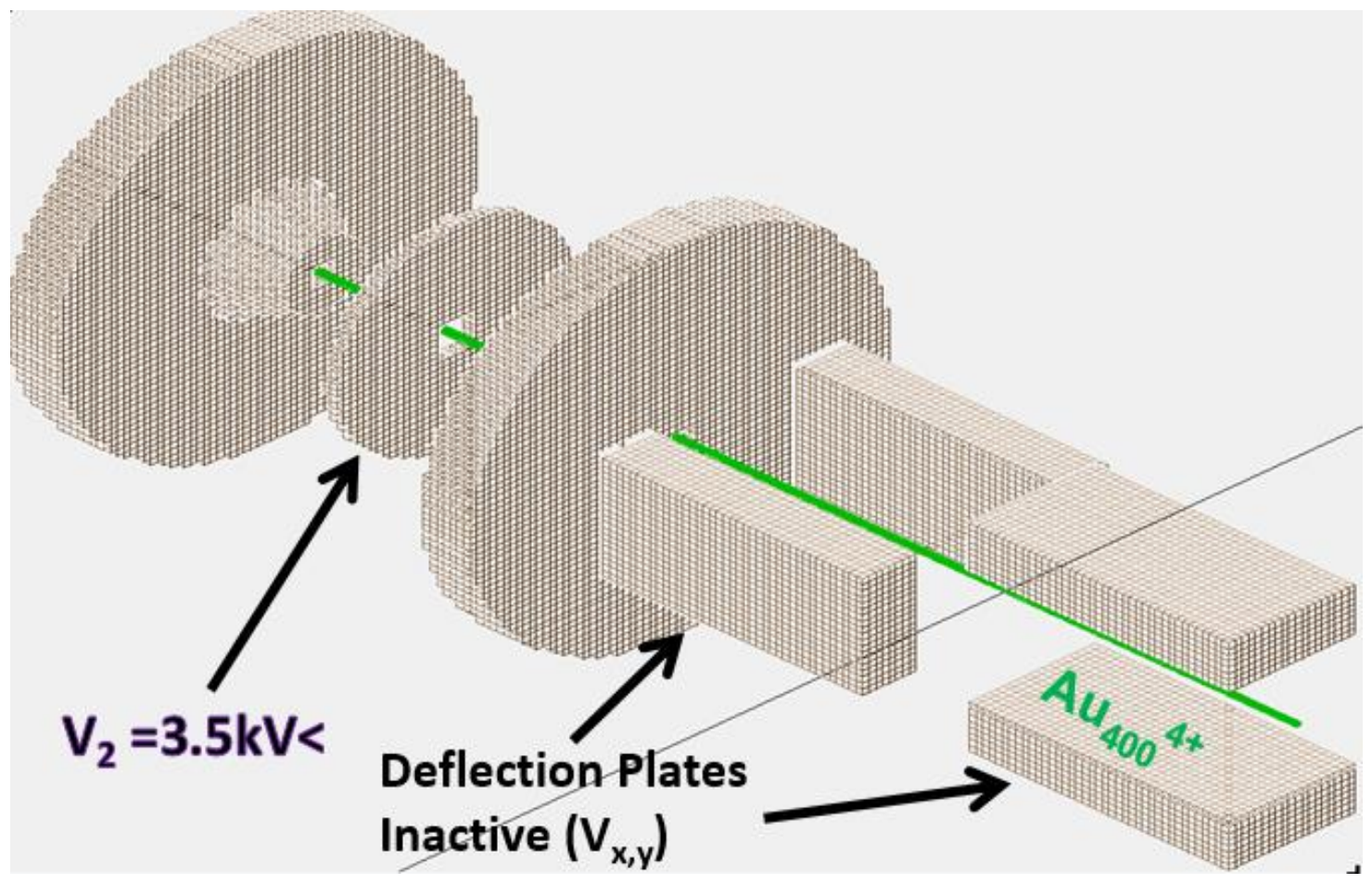

Figure 18. 3D view of post-filter einzel lens and electrostatic plates (deflection/pulsing) simulation performance with components highlighted. 


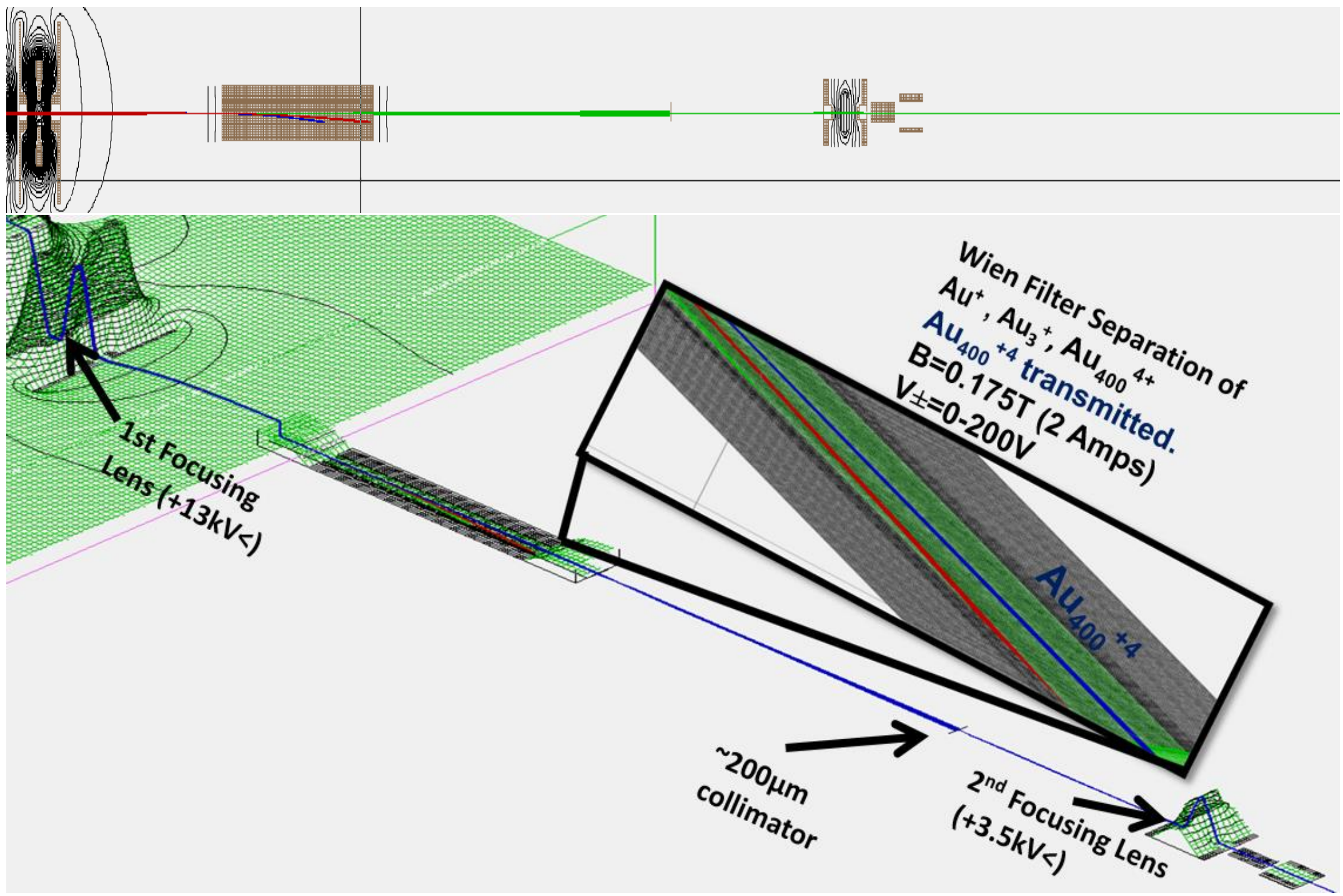

Figure 19. 2D XY and 3D simulation of the potential energy lines and surfaces for primary ion column components. 


\subsubsection{Finalized Assembly of the Primary Ion Column and Further Modifications}

The manufacturing and fabrication of the components was custom-built in Florida International University - Physics Department and the Texas A \& M University Chemistry Department Machine Shops. The raw materials (e.g., steels, aluminum, insulating materials, wiring and wieldable connectors) were purchased from McMaster Carr (U.S.). Specialized high voltage, motion control, vacuum feedthroughs and flanges were purchased from Kurt Lesker (Jefferson Hills, PA) and MDC Vacuum (Hayward, CA).

Initial fabrication consisted in the housing chambers for the source and pre-filter einzel lens, the Wien Filter and the diagnostic chambers. The housing was fabricated from a .316" ground steel block (McMaster Carr). Following this, the electrodes and apertures for the lens were fabricated from .316 " ground steel along with the insulating PEEK (Poly Ether Ketone) spacers (McMaster Carr). The middle electrode is biased from Mastsusada AU-20 kV high voltage supply via a CF600 SHV tri-feedthrough flange (MDC Vacuum.) The SHV cables for high voltage feeding were fabricated in-house from cabling and connectors provided by Matsusada (San Jose, CA). The main housing structure was made of a 6000 class-high corrosion resistance aluminum-magnesium alloy (McMaster Carr). Features on the housing are two CF600 flange mounting for bridging the source and diagnostic chambers, along with the feedthrough apertures for welding the BNC connectors to bias the electrodes. The mounting for the copper solenoids and Teflon housing used in generating the magnetic field extrude from the main structure. The solenoids consist of roughly $\sim 65$ spins of 16 gauge $95 \% \mathrm{Cu}($ w/wt $\%$ ) copper wire (McMaster Carr). These Teflon shields are mounted within 430F ferromagnetic steel 
blocks, where the fields are generated (McMaster Carr). These are connected to a TDKLambda current generator via MOLEX feedthroughs.

Assembly of the outer parts of the Wien Filter took place afterwards, with the solenoid housing, magnets, and solenoids being installed. The BNC feedthroughs connectors to bias the electrodes and MOLEX connectors utilized to supply current for the solenoids were soldered in-house. Lastly, the electrode rails were fabricated from high purity copper (>95\%, McMaster Carr). Two different types were made, electrodes with a focusing rectangular geometry and conventional flat electrodes. This was done in order to compare the performance both theoretically (SIMION) and experimentally. Work on the diagnostic chamber began by the fabrication and assembly of the first set of $\mathrm{XY}$ deflector plates at the entrance of the diagnostic chamber. Housing for this component was custom-ordered, fabricated made from.316 stainless steel (Kurt Lesker).

The diagnostic chamber collimator array and XY controllable mounting for collimating aperture size selection were fabricated and assembled afterwards. CF275 mountable XY controllers for adjusting the collimator selection were purchased from MDC. The last set of fabricated components were those for the post-filter einzel lens and $2^{\text {nd }}$ deflector set consisting of the PEEK mounting rods, electrodes, $2^{\text {nd }}$ set of XY deflector plates, and PEEK spacers. The electrodes were made from .316 stainless steel and mounted on PEEK supports, separated by ribbed PEEK spacers, and with the second electrode shielded by a PEEK enclosure (McMaster Carr). A $20 \mathrm{kV}$ Matsusada DC power supply feeds to the second electrode, yet this lens system is intended to operate at significant lower voltages compared to its larger counterpart. The extractor plates are 
constructed from .316 stainless steel as well, with PEEK spacers separating the lens, and the two sets of plates (McMaster Carr).

\section{Vacuum system and Upgrades.}

The high vacuum of the PI column is achieved using two Edwards EXT 255HP turmobolecular vacuum pumps. The turbo controllers were built at the Florida International University Physics Department Electronics Shop. Rough pumping is performed with two Edwards RV 12 HP oil-sealed two stage rotary vane pumps connected to each turbomolecular pumps. Vacuum pressures are controlled using Edwards brand Pirani Gauges and Bayard Alpert (hot filament) gauges in the source chamber and diagnostic chamber, respectively.
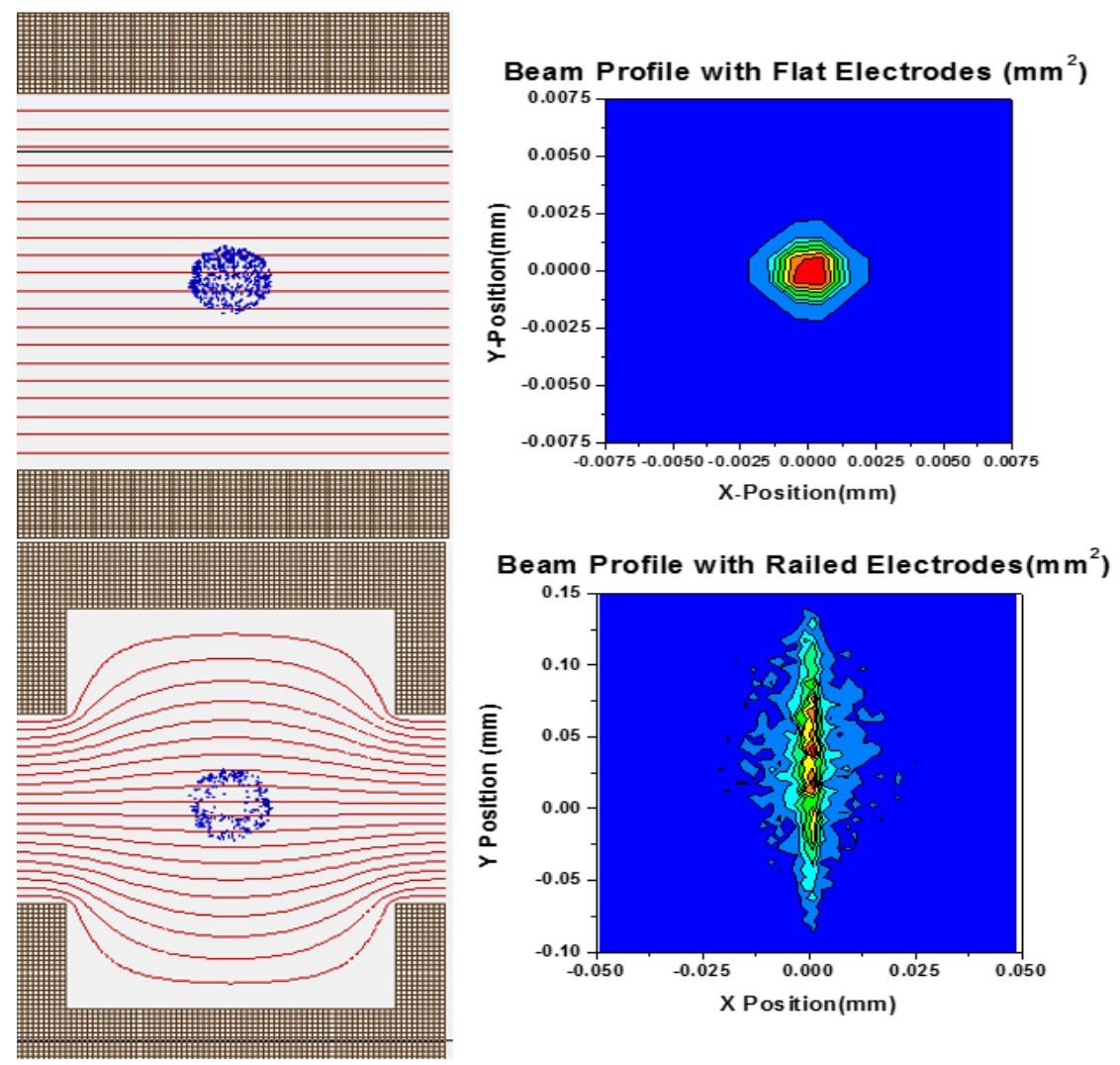

Figure 20. SIMION analysis for comparing beam cross-sections based on Wien Filter electrodes' geometry. 
The gauges are connected to an Edwards pressure readout interface, where different gauges can be monitored. During experimental testing, several upgrades were performed to improve the column operation. For example, the original stage (Thermionics EC-XY B275C) was switched for a new Thermionics FB XYZ-B6T CF600 high precision stage $(0.0001$ ") for more robust and precise motion controllers. The Wien Filter design uses flat electrodes, with SIMION simulations demonstrating the elimination of the beam axial spread (Figure 20). The arrangement of the turbomolecular vacuum pumps was modified to increase life time and column stability. 


\section{Initial Design (2013-2014)}

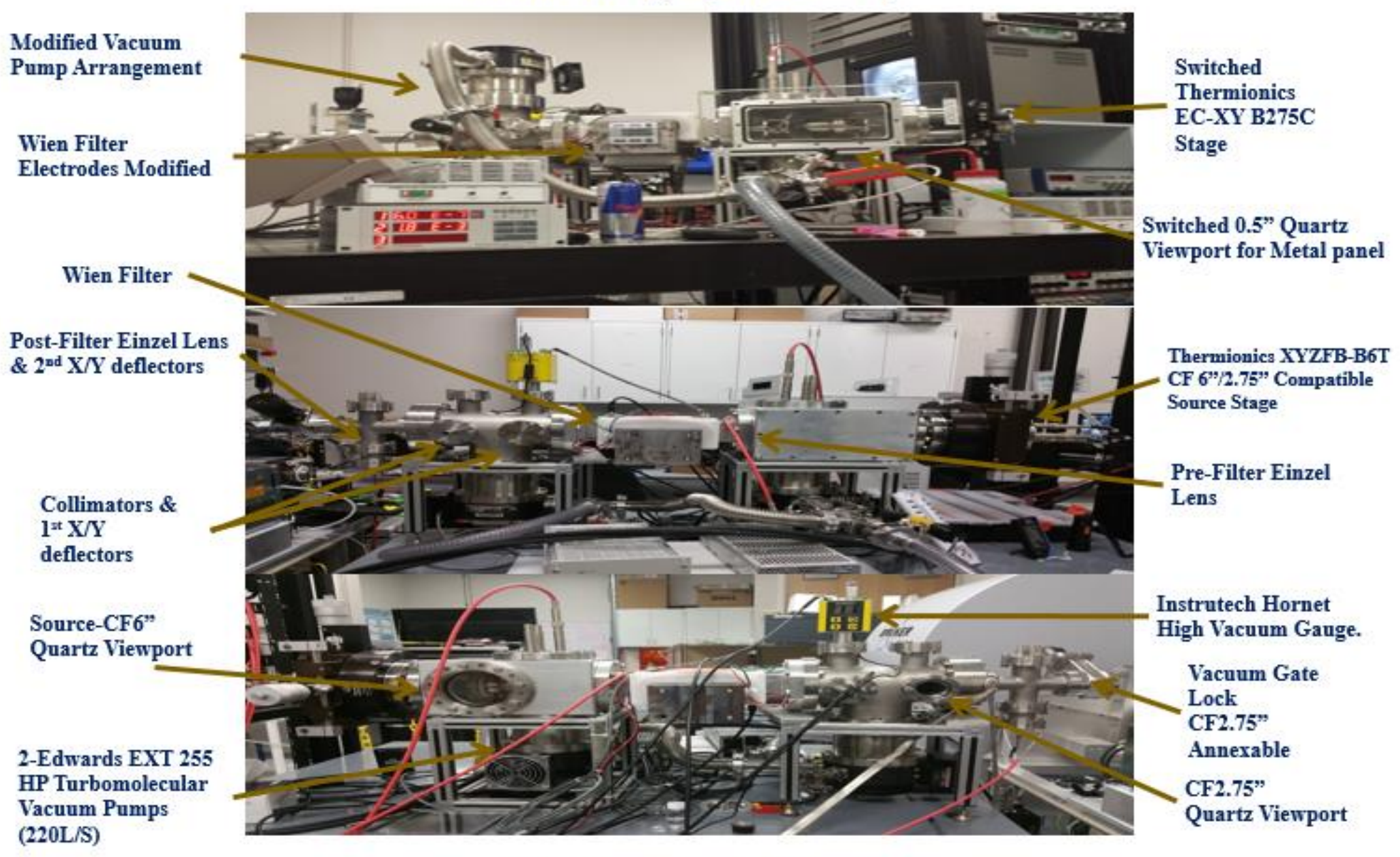

Final Design (2014-2015)

Figure 21. Side view of initial and final primary ion column design with key differences and features highlighted. 


\subsection{Conclusions}

The design of a multi-source PI column was completed employing a combination of computer assisted design tools and theoretical ion dynamic simulations. The design goals consisted of building a sturdy, robust array of focusing optics to provide a variable degree of beam focusing parameters for both static and dynamic SIMS applications. A combination of two defocusing-focusing einzel lens were designed and theoretically elucidated to generate a beam with the potential of reducing the cross-section of ion beams down to $10 \mu \mathrm{m}$ or less, allowing this PI column to probe nanoscale objects. Lens design allows for a shift in focusing strength and length in order to effectively maximize PI transmission for a dynamic SIMS type analyses with such column.

A Wien filter was also designed and theoretically described to separate and transmit only ions of a desired $\mathrm{m} / \mathrm{z}$. The Wien filter permits an experimental assessment of projectile distributions for both known and novel PI sources. In combination with simulations, exact values for consistent projectile transmission can be obtained and applied to experimental settings to determine the $m / z$ of unknown projectiles in novel sources. A set of beam collimators was designed to reduce the beam diameter and reduce the effect of charge-repulsion diffraction and occurrence of geometrical aberrations. This PI column was also equipped with a set of two XY electrostatic plates for both beamline alignment, beam pulsation and TOF measurements for projectile characterization, intandem or independent of the Wien filter operation. 


\section{EVALUATION OF THE AU-LMIS AND INCORPORATION TO THE COLUMN}

\subsection{Introduction}

Following the completion of the PI column, in this section, we describe the integration of AuNP-LMIS to the column. As discussed in the introduction, massive gold nanoparticles $\left(\mathrm{Au}_{400}{ }^{+4}\right)$ projectiles have shown advantages over other type of projectiles. Preliminary designs for this type of source date to the early 1990's, where the first polyatomic cluster projectiles were studied to evaluate the benefits in employing these as opposed to monoatomic projectiles ${ }^{69}$ Dr. Serge Della-Negra, along with Dr. Emile Schewikert participated in most of the ground work for the early stages of Au-LMIS development for SIMS applications ${ }^{73,74}$. Early work at the Institute of Nuclear Physics (Orsay, France) aimed to characterize the distribution of ions generated from gallium and gold LMISs. Upon steady generation of cluster projectiles, SI yields and enhancement factors were calculated on a variety of targets, particularly metallic, semiconductor and organic targets (see Figure 22) ${ }^{73}$.

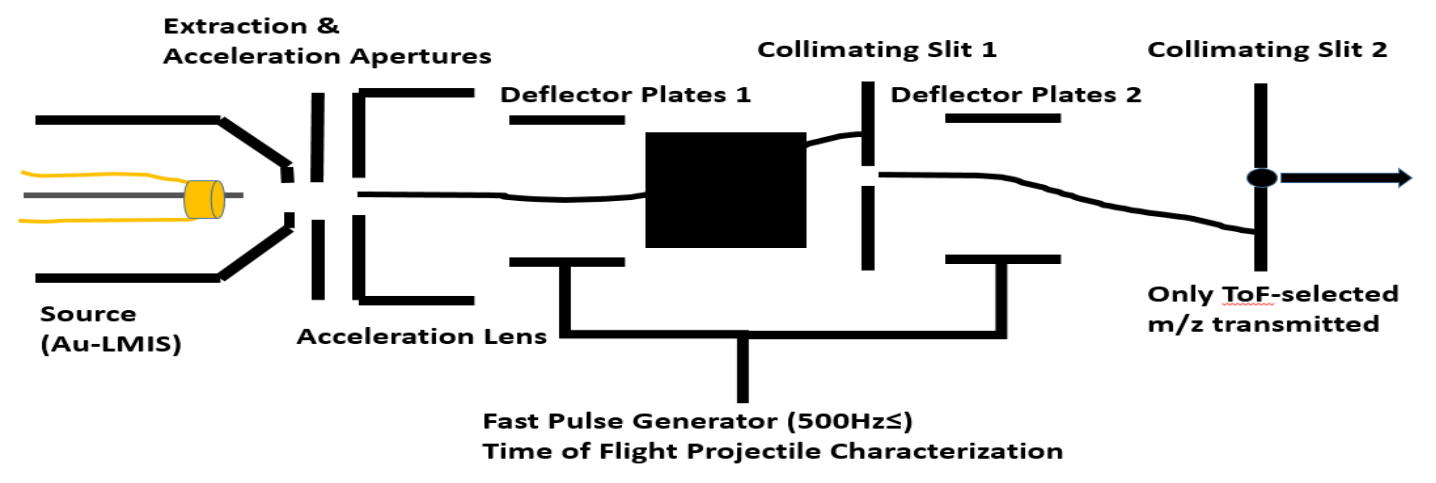

Figure $22^{73}$. Schematic of primary ion column for the characterization of Au cluster projectiles utilizing time of flight measurements. Reprinted with permission from M. Benguerba. Copyright 1991 Elsevier B.V. 
Experiments showed that the Au-LMIS is capable of generating a distribution of clusters $\mathrm{Au}_{\mathrm{n}}{ }^{\mathrm{q}+}$, where $\mathrm{n}=1-7$ and $\mathrm{q}=1,2$. It was noted that the prevalence of these cluster constituents decreases with size (Figure 23). Upon characterizing the beam, specific projectile sizes were chosen to evaluate the ionization yield enhancements compared to monoatomic projectiles and between varying Au cluster sizes ${ }^{76}$.

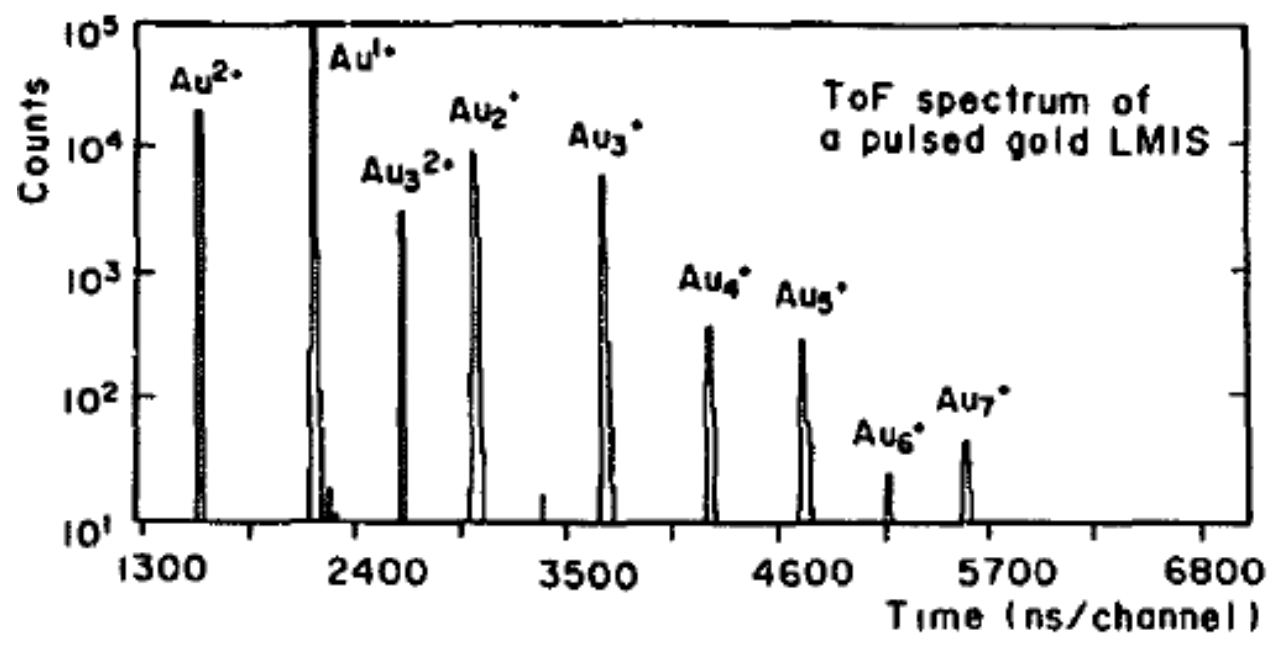

Figure $23^{73}$. Results from the Au projectile TOF characterization experiments highlighting different cluster sizes $(n=1-7)$. Larger projectiles have greater times of flight. Reprinted with permission from M. Benguerba. Copyright 1991 Elsevier B.V.

An important parameter in these experiments was variability in initial PI energy, where the source voltage was changed, thus influencing the energy per mass unit, or energy/nucleon. SI yield enhancements were measured based on the comparison of desorption and ionization yields of individual gold cluster impacts as opposed to the individual constituents of that projectile. This was done in a fashion akin to the CsIpolyatomic beam experiments ${ }^{74}$, where yields from polyatomic species were compared against their individual constituents per constituent unit in an event-by-event mode This work concluded that ionization enhancements were significant, with very clear evidence of a non-linear enhancement trend between monoatomic and cluster projectiles. 
Evidence of this comes from the ionization yield enhancement observed on a phenylalanine target shown (Figure 24), where the molecular ion yield increased by a factor of 30 between $\mathrm{Au}^{+}$and $\mathrm{Au}_{3}{ }^{+}$impacts ${ }^{73}$. The rate of change in yields remains linear with respect to energy, yet experiences a gradual increase for each additional constituent. Another prevalent trend was that the ratio of molecular ions to fragments yielded a preferential generation of fragments. Results show that favored molecular ion generation was increased with larger projectile size ${ }^{76}$. Previous work also involved the usage of the ORION High Energy Particle Injector at IPNO in order to study the different mechanistic aspects of cluster bombardment at $\mathrm{MeV}^{70}$.

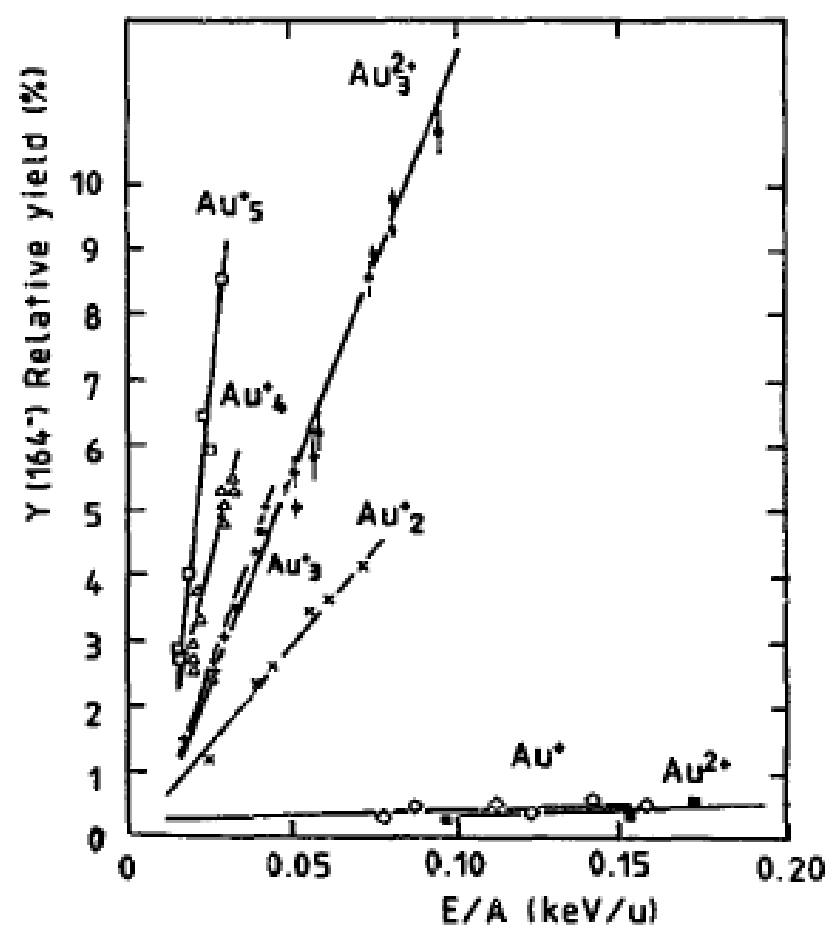

Figure $24^{73}$. Comparison of secondary ion yields between gold projectiles $n=1-5$, $q=1-2$ as a function of projectile kinetic energy. Reprinted with permission from $M$. Benguerba. Copyright 1991 Elsevier B.V. 
In 2004, Dr. Serge Della-Negra and coworkers profile the distribution of PI species generated from an Au-LMIS using a system analogous to that presented in Figure 22. Briefly, the system consisted of a source, a set of focusing electrostatic lens, a Wien Filter, as well as a High Energy Ion Injector (ORION Injector) for MeV ion impacts employed in the initial source experimentation. In other theoretical and experimental work it was found than an emitter angle of $\left(90^{\circ}\right)$ led to the preferential generation of larger charged species as opposed to emitters with more acute angles (i.e. $\left.60^{\circ}\right)^{99}$. Molecular Dynamics simulations indicate that the point-source for projectile generation allows for droplets to "pinch" off, rather than the traditional generation of a steady-stream of smaller charged species $\left(\mathrm{M}^{+\mathrm{q}}\right)$ species. This geometry comes with a trade off in that a wider conical angular distribution occurs as opposed to emitters with sharper angles, leading to a more challenge focusing of the beam. The sizes and charges of the projectiles were measured employing ion mass selection (Wien Filter) in conjunction with multiplicity and TOF event-by event calculations ${ }^{99}$. The Wien Filter served as a PI filter for separating ions based on their velocity, however unable to separate ion with the same $m / z$. Mass-selected incident projectiles would impact a Formwar foil, providing a start signal and secondary electrons for energy measurements to a Multi-Channel Collector Plate (MCP) and oscilloscope to verify the projectiles would not disintegrate in flight. $\mathrm{Au}_{\mathrm{n}}{ }^{\mathrm{q}+}$ fragments generated after collisions would have their flight time, from the foil to a set of multianode detectors, measured along with their energy losses (dE). These were measured from the velocity of the incident impact, post-collision fragments and secondary electrons to generate an overall distribution of exact $\mathrm{n} / \mathrm{q}$ based on the WienFilter mass selection window. Other particular variables of interest were the overall 
extraction current, its influence on the generation of cluster projectiles, and its relation to source lifetime, as greater source brightness requires a faster expenditure of the source material (>95\% Au: 5\%> Si eutectic).

\begin{tabular}{|c|c|c|c|c|c|c|c|c|c|c|c|}
\hline & $\mathbf{q}$ & 1 & 2 & 3 & 4 & 5 & 6 & 7 & 8 & 9 & $q_{\text {mean }}$ \\
\hline & $n / q$ & & & & & & & & & & \\
\hline \multirow[t]{6}{*}{ Low Charge } & 5 & 98.7 & 1.3 & & & & & & & & 1.01 \\
\hline & 7 & 94 & 6 & & & & & & & & 1.06 \\
\hline & 9 & 96.6 & 3.4 & & & & & & & & 1.03 \\
\hline & 27 & 24.6 & 18.8 & 19.2 & 15.5 & 13.8 & 8.1 & & & & 3 \\
\hline & 40 & 1 & 11 & 36 & 44 & 7 & & & & & 3.4 \\
\hline & 120 & 3.1 & 7.4 & 16 & 20.9 & 18 & 13.4 & 10.3 & 6 & 4.7 & 4.8 \\
\hline \multirow[t]{3}{*}{ High Charge } & 9 & 23 & 74 & 3 & & & & & & & 1.8 \\
\hline & 27 & 4.1 & 13.3 & 26 & 34.8 & 16.6 & 6 & & & & 3.6 \\
\hline & 40 & 2 & 4.5 & 12 & 25 & 31 & 19.5 & 6 & & & 4.6 \\
\hline
\end{tabular}

Figure $25^{99}$. Measured distributions of cluster sizes (n) as a function of the amount of charges $(q)$. Note that the heavier the cluster the more charges are available. Reprinted with permission from Della-Negra. Copyright 2004 Elsevier B.V.

Results, shown in Figure 25 showed a wide distribution of charged species with sizes spanning $n=1$ to $n=1000$ with charges as far as $q=+9$. A particular region of interest is found at Wien electrode voltages $\mathrm{V}_{\text {Wien }}<20 \mathrm{~V}$, where large species containing $\mathrm{n}=27$, $n=40$, and $n=120$ constituents generated with consistency, with some room for variance around these distributions. A region of projectiles of interest is found around $n=120$, where projectiles can thus be characterized as massive, consisting of liquid nanoparticles with many constituents. For this projectile size, the charge distribution (q) is 4.8 , with 5 . This work concluded that Massive Gold Nanoparticles $\left(\mathrm{Au}_{100}{ }^{+\mathrm{q}}\right)$ can be generated steadily and employed for SIMS applications were the generation of molecular ion species was the primary goal.

Further work by Dr. Emile Schweikert's group utilized the event-by-event method with single $\mathrm{C}_{60}{ }^{+}$and massive gold cluster impacts to perform nanoscale chemical 
mapping as a function of projectile size in the PEGASE platform ${ }^{101,102}$. The PEGASE platform is a bi-direction TOF-SIMS equipped with a 100-150 kV PI accelerator developed at the IPNO equipped with an electron emission microscope (Figure 26). This allowed for an acquisition of MSI data in a format comprised of ion density plots, as well as electron emission co-location for nanoscale mapping of these individual impacts ${ }^{102}$. With a beam fluence of $1.0 \times 10^{9}$ ions $/ \mathrm{cm}^{2}$, each impact interrogates an unperturbed area of the surface, making the spatial resolution of the probe equivalent to the volume of emission by projectile impact, which through AFM observations was found to be about $10 \mathrm{~nm}$ for massive gold cluster impacts ${ }^{140}$.

Secondary electron localization relies on a fast frame-recording CMOS camera, from which the electron-based surface mapping is performed. Operation of this instrument requires a highly synchronized set of electronics, starting with the detection of individual PI impacts on the target. From there, a weak magnetic prism deflects electrons into a set of aberration reduction optics, followed by a set of magnifying ion optics. The secondary electrons impact the custom-made position-sensitive detector (PSD) which has an aluminized phosphorous, with an emission decay time of $100 \mu$ s. Impact and subsequent photon emission acts as the start signal for CMOS camera to collect an image of the electron location based on the fluorescence from the phosphorous screen. Upon synchronized triggering of the PSD and the camera within a certain coincidence time window, a start signal is provided to the TOF buncher to pulse the SIs into the TOF analyzer. A time-to-digital converter controls the overall intervals between start and stop signals based on the number of discrete camera shots per secondary electron impact and subsequent image detection process. This event-by-event approach thus allows for the 
collection of an individual mass spectrum corresponding to each individual projectile impact $^{103}$. While having the ability of resolving discrete impacts at a distance of less than $100 \mathrm{~nm}$, the mass resolution of this instrument is $\sim 4000(\mathrm{M} / \Delta \mathrm{M})$ allows for identification of small molecules from the surface of interest ${ }^{100}$.

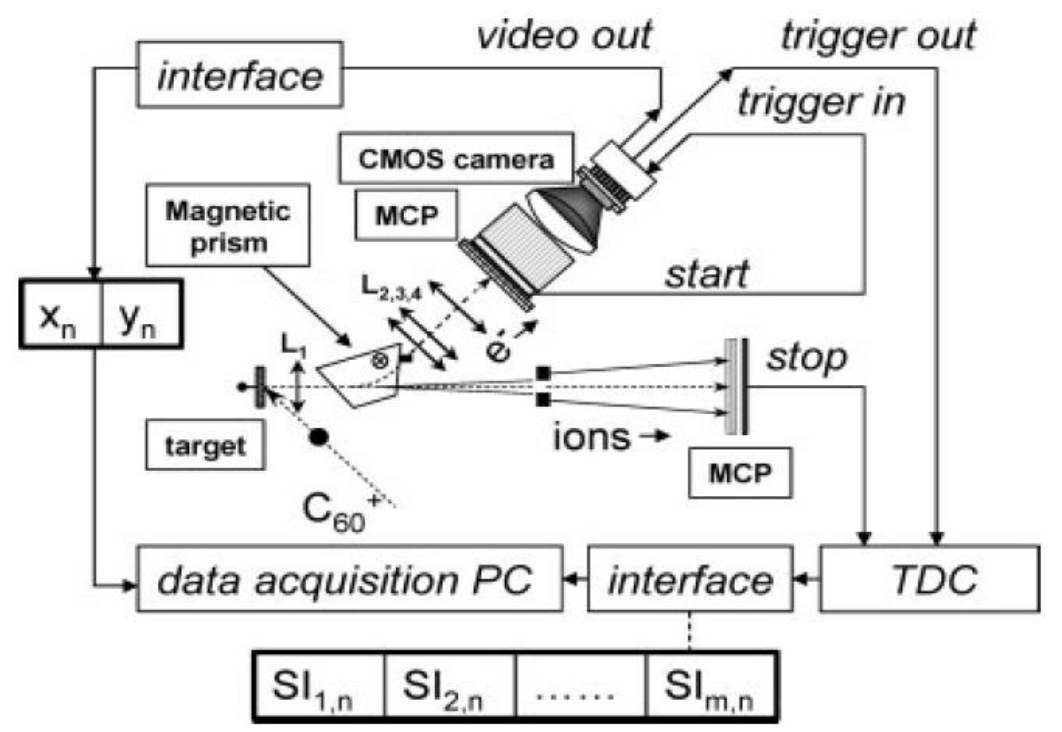

Figure $26^{101}$. Schematic for the ion and electron optics on the PEGASE instrument demonstrating sequence of signaling for single-impact detection. Reprinted with permission from FFL et al (2012). Copyright 2012 American Chemical Society.

3.2 Methodology for Source Incorporation and Testing

\subsubsection{Design Adaption and Fabrication}

The Au-LMIS was based on general schemes for modern LMISs, where a micrometric tungsten emitter has a metal eutectic filled reservoir, and this is located at a distance of $0.5-2 \mathrm{~mm}$ from a set of extraction optics ${ }^{56}$. The metal is melted by the usage of a current on the filament, and the liquid metal then creeps towards the edge of the emitter due to the large electrostatic repulsion generated by the high ionization voltage. This leads to the formation of an electrified interface (Taylor cone), from where PIs are extracted into the PI column. 
The emitter consists of a set of three filament supports, where the two lateral elements hold the reservoir filament and the central holds the emission filament ${ }^{140}$. The feed through for these three supports comes from the rear part of the source, where electrical connections are made (Figure 27). In order to avoid discharging, a significant concern for source stability, the feedthroughs are insulated and structurally supported with an insulating ceramic (Macor) base. The extractor array consists of a set of concentric, cone-shaped electrodes in order to promote electrostatic field homogeneity, ensuing the generation of a uniform Taylor cone.

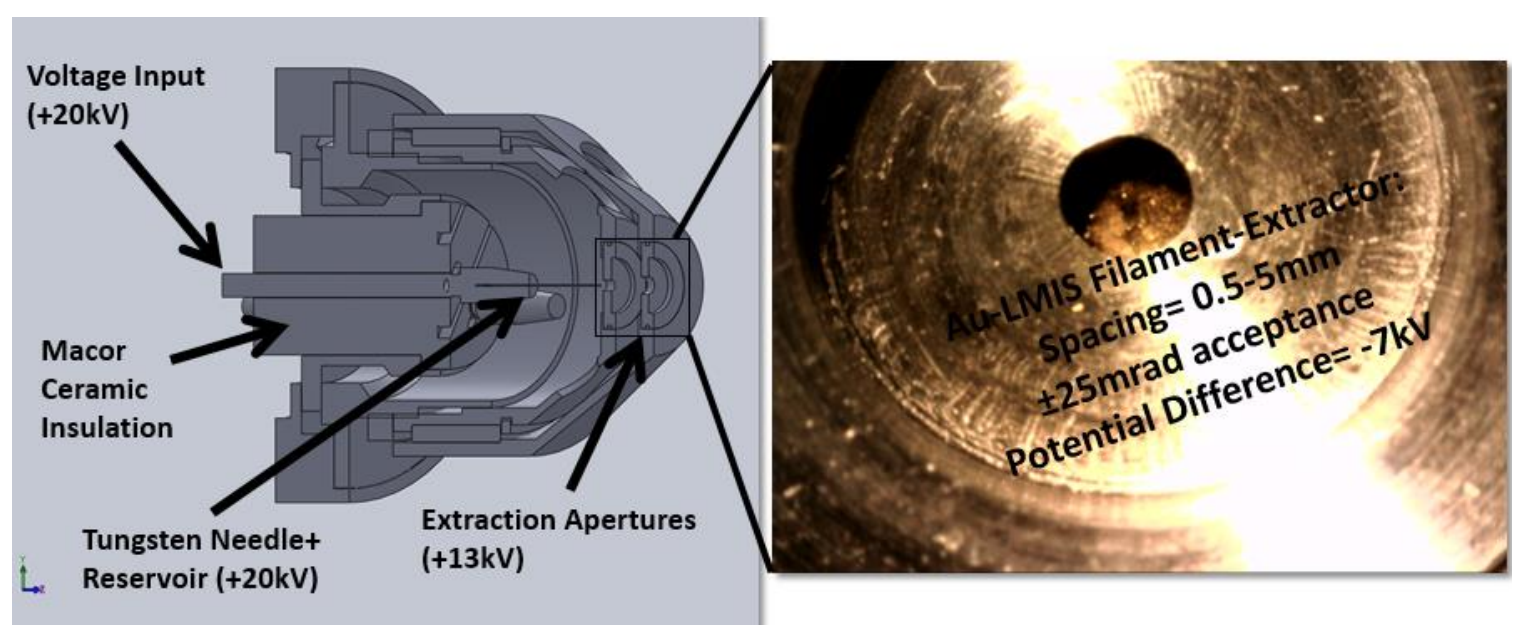

Figure 27. 3D cross-section view of Au-LMIS structure, with the insert highlighting a top view perspective of a prepared emitter utilized in characterization experiments.

The extraction apertures are friction-fitting rather than welded on, as these become sputtered during source operation causing asymmetry in the aperture. By replacing them upon evident wear (aperture asymmetry), angular distribution irregularities can be avoided. Beyond the structural features, something else to be taken into account is the potentials at which the source was operated and how to supply these potentials. The source potential is to be held at $20 \mathrm{kV}$, while the extractor is to be operated at $13 \mathrm{kV}$. A 
current supply is also required in order to melt the Au-Si eutectic within the reservoir in order for the gold to be able to creep towards the end of the emitter. These electronics require specialized, insulating housing. The housing was fabricated from Telfon and Plexiglass, and is shown in Figure 28 with the enclosed electronics inside.

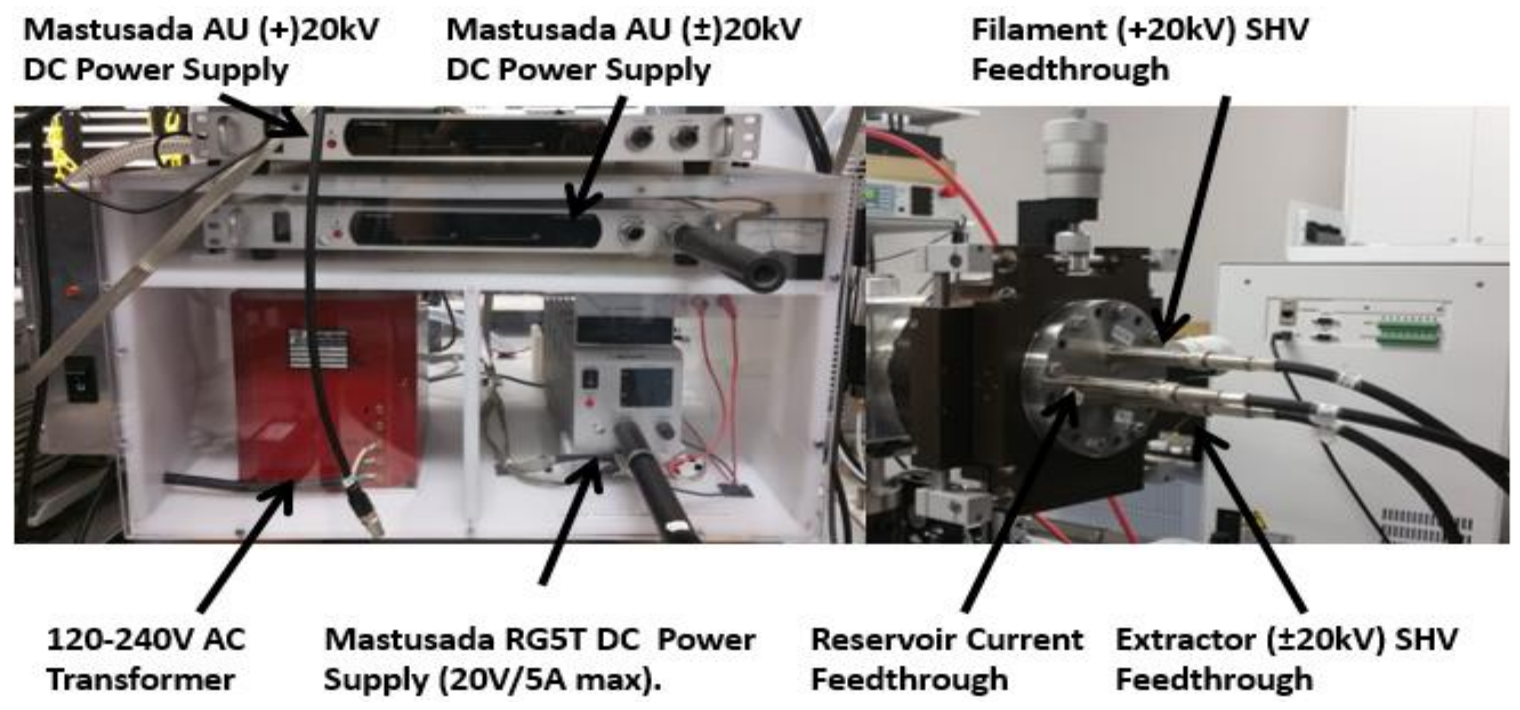

Figure 28. Specialized electronics for powering the $20 \mathrm{kV}$ LMIS inside the primary ion column with components and connections highlighted.

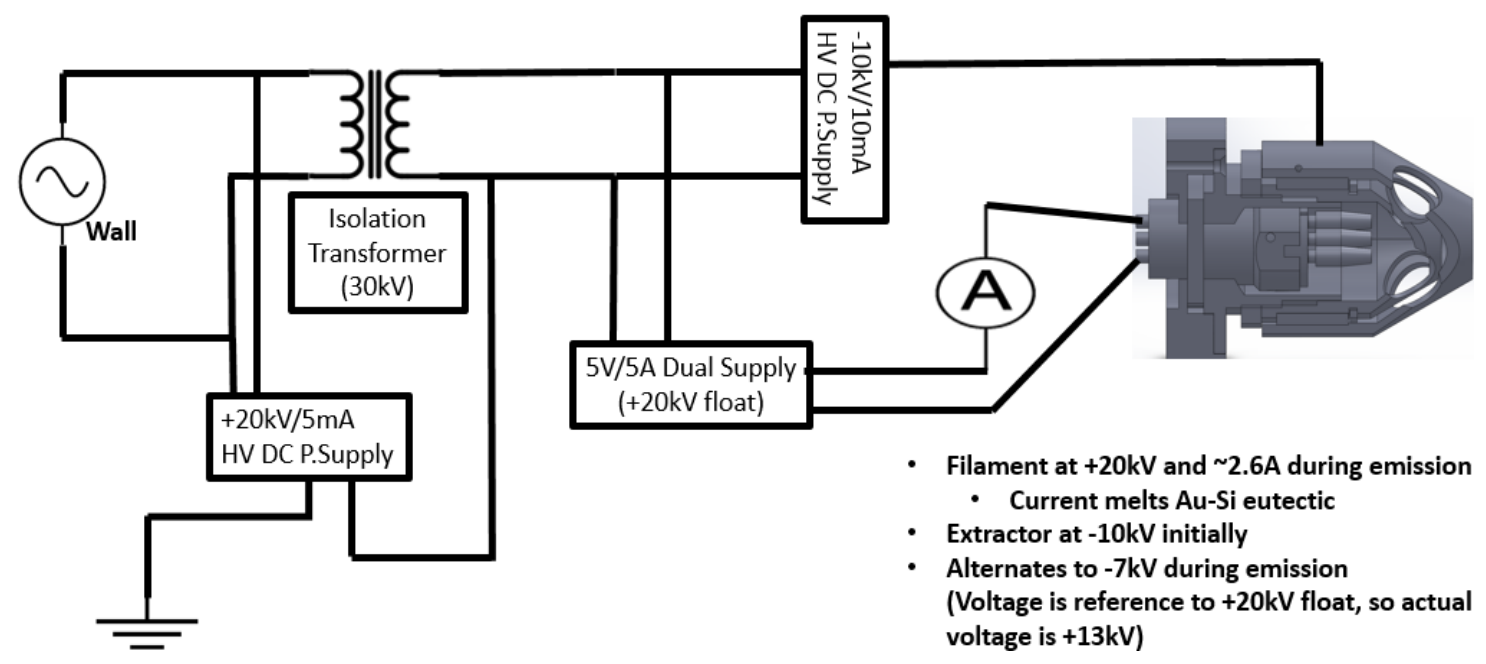

Figure 29. Electronics wiring diagram for the LMIS. 
A $120 \mathrm{~V}$ to $240 \mathrm{~V}$ AC isolation transformer powers the filament current supply, and the extractor supply while providing a bias from the external power supply (Figure 29). The power supplies selected are Matsusada AU series modular (+/-) $20 \mathrm{kV} / 10 \mathrm{~mA}$ power supplies and the power supply for the current feed is a Matsusada RG5T DC. Holes on the front face of the housing permit manipulation of potentials, and emergency shut down of individual supplies. The supplies are controlled manually using a high-density insulating nylon rod. Given the thickness of the tungsten filaments utilized to make these sources, currents employed generally are less than 5A. Prior work with the Au-Si eutectic that is utilized in this source gives its melting range to be $240-250 \mathrm{C}^{\mathrm{o}}$ at sea-level in a vacuum ( $>1.0 \times 10^{-5}$ torr, Sigma Aldrich Filaments). Currents of 2.4-2.6 A are utilized to melt the gold eutectic without structurally compromising the emitter filament. The filament preparation and fabrication procedures is found in Appendix 1. Generally, a spacing of $5 \mathrm{~mm}$ or less is desired between the emitter and the extraction aperture. The spacing value is of importance experimentally, based on the voltage to be utilized on the emitter, in order to avoid discharging and sustain beam stability. The acceptance angle in our current source design is that of $25 \mathrm{mrad}$ above and below the axial line at $0.5 \mathrm{~mm}$ spacing.

Mounting for the LMIS was modified from a $25 \mathrm{~mm}$ thick silicide-type ceramic rod attached to a circular disk where the connectors from the feedthroughs would directly feed the source to $50 \mathrm{~mm} .316$ steel rod mounted on a CF600" flange, along with a pivot point added so that the source could be removed without the need of undoing connections (Figure 30). The connectors were also modified from direct contact (screw-in) for pushpin based connectors (pressure/contact based). This was done in order to reduce problems 
related to discharging from the fixed connectors onto adjacent conductive sites and to facilitate source switching without having to undo connections.

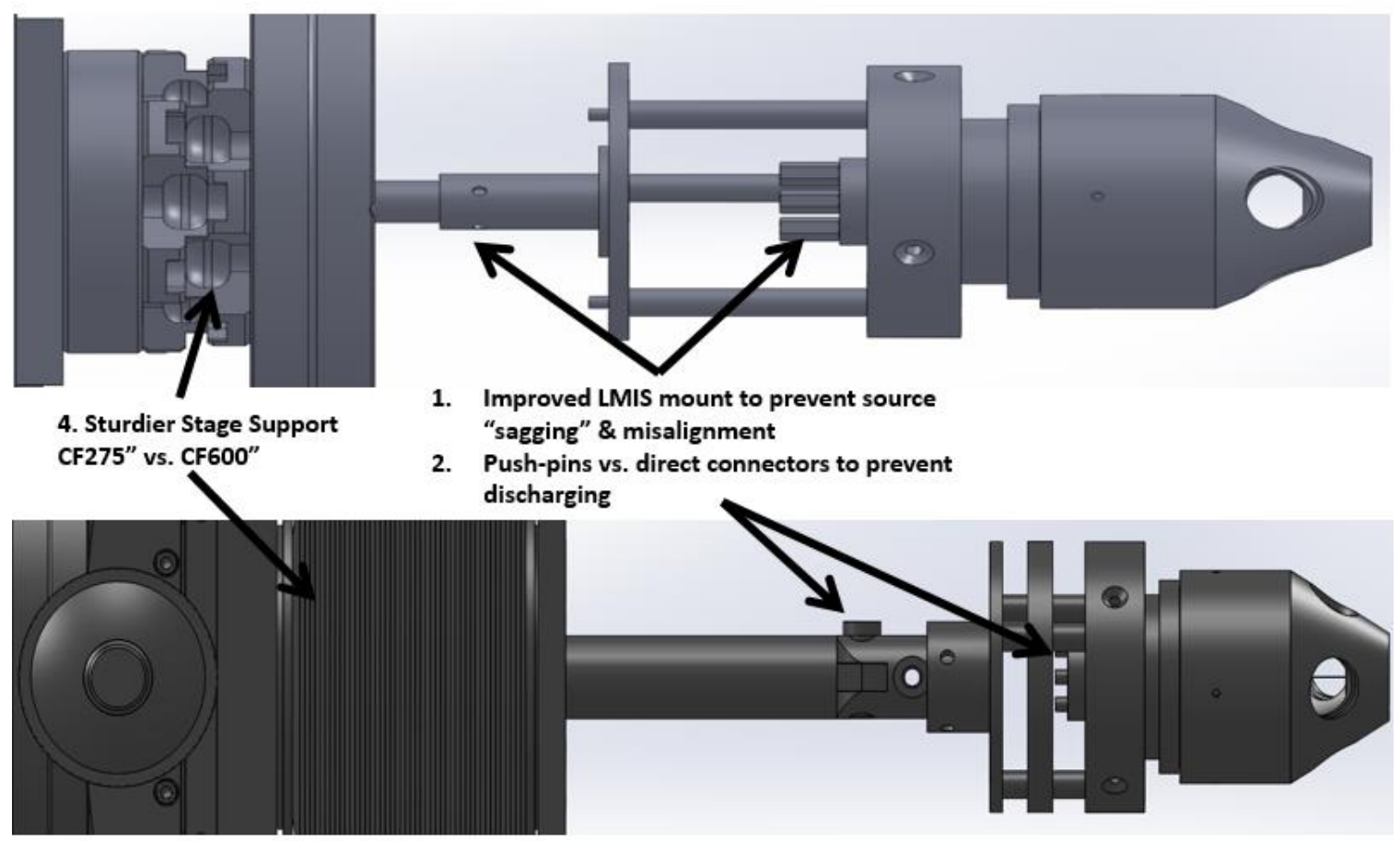

Figure 30. Comparison of early and current LMIS structure, mounting systems, and feedthrough.

\subsubsection{SIMION Simulation of Ion Trajectories}

The simulation of gold cluster trajectories was performed employing a set of PI conditions comparable to those in operation of normal LMIS (Figure 31). The $\operatorname{Au}_{100(q)}{ }^{(q)+}$ projectiles had their mass based around a mean of 400 constituents with a deviation of up to \pm 10 consituents (1970 FWHM) to account for minor variance in projectile generation.

A fix charge value of $q=4$ was given because the main projectiles of interest are $\mathrm{Au}_{400(\mathrm{q})}{ }^{(4)}$, for which $\mathrm{q}=4$. Initial ion position takes account the radius of the Taylor cone formed, and the acceptance angle limitations of the extractor. Thus, ions are generated 
around a $\theta=25 \mathrm{mrad}$ angular distribution and with an initial gaussian distribution conical spread angle of $\pm 30^{\circ}$.

Ions were given a fixed kinetic energy of $0.1 \mathrm{eV}$ upon generation. Other features like charge weighing factor were left intact as these projectiles have such high velocities that repulsion energies become negligible.

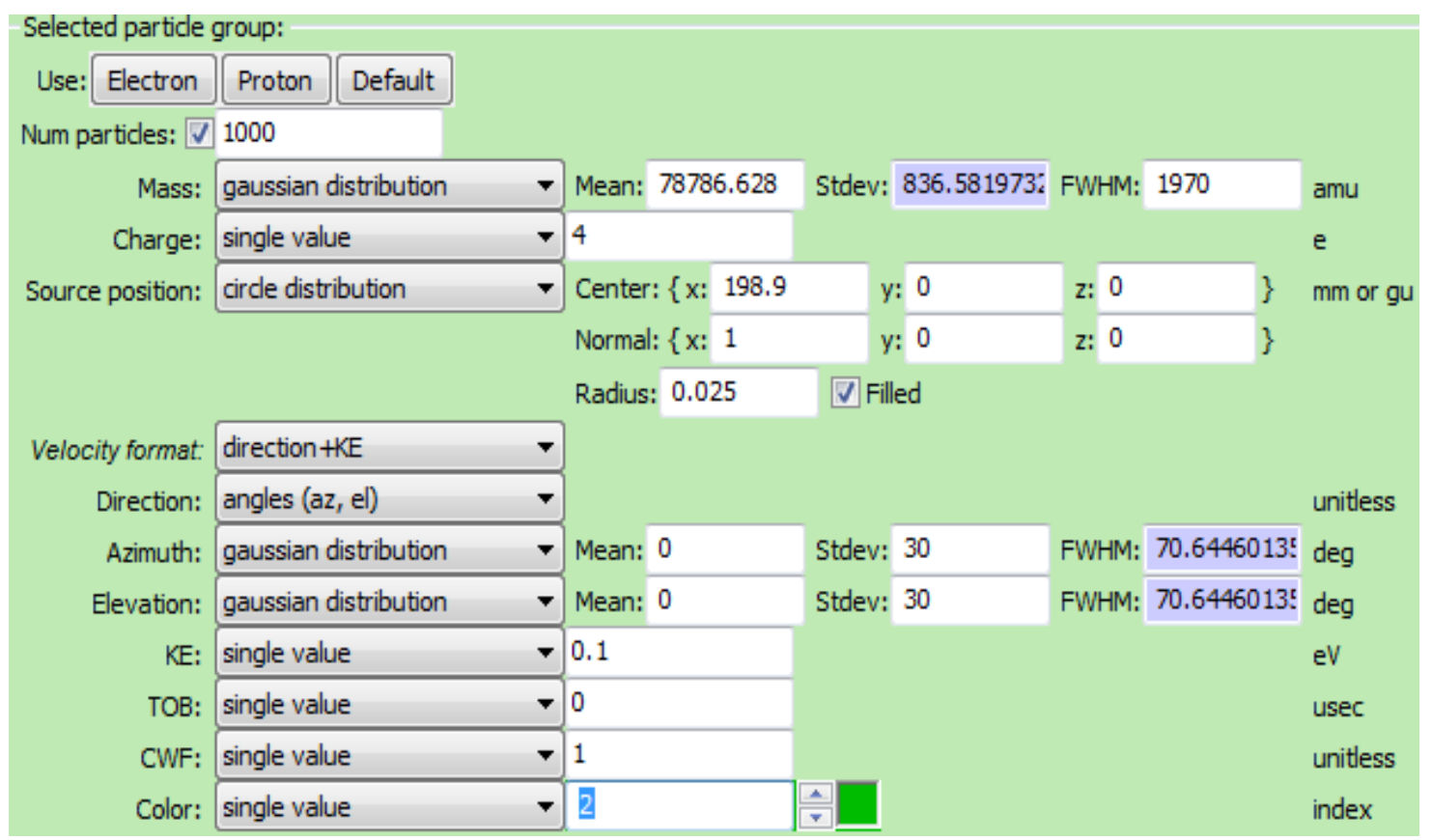

Figure 31. Primary ion generation and trajectory conditions for simulation.

The main input for kinetic energy comes from the filament, which is charged to $20 \mathrm{kV}$, granting all ions with a initial kinetic energy of $20 \mathrm{qkeV}$. Extraction optics were set to 13 $\mathrm{kV}$, and placed at $2.5 \mathrm{~mm}$ from the filament to create an overall extraction potential of 7 $\mathrm{kV}$. The source physical array was overlapped with that of the pre-filter einzel lens. The theoretical performance for ion beam extraction, subsequent acceleration, and focusing is illustrated in Figure 32. 


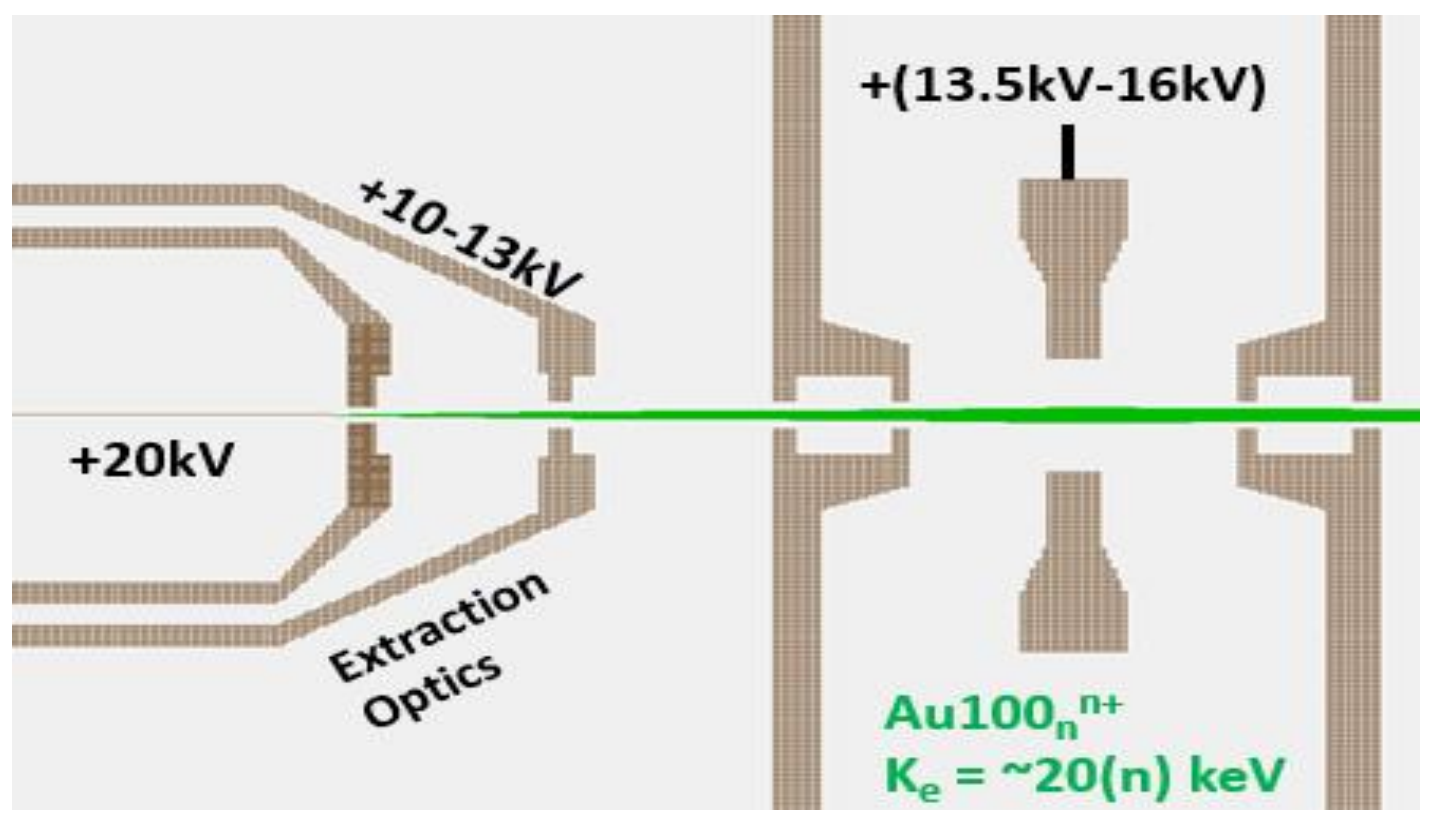

Figure 32. XY simulation view of the Au-LMIS and pre-filter einzel lens illustrating the trajectory of the primary ions upon generation and subsequent emission.

Figure 33 displays the overall ion optics workbench created for these simulations.

Potential values for the components can be optimized in order to achieve two different types of criteria: i) a high current and ii) a high focus. In the high current there is minimal PI loss, yet a beam diameter in the hundreds of micrometers is obtained. This constriction in beam diameter can be overcome by employing an event-by-event mode if high spatial resolution is desired. The other criteria is the ability of reducing the beam cross section to $>10 \mu \mathrm{m}$, allowing for the usage of massive gold clusters as a pulsable, nanodomain analysis static SIMS probe. 


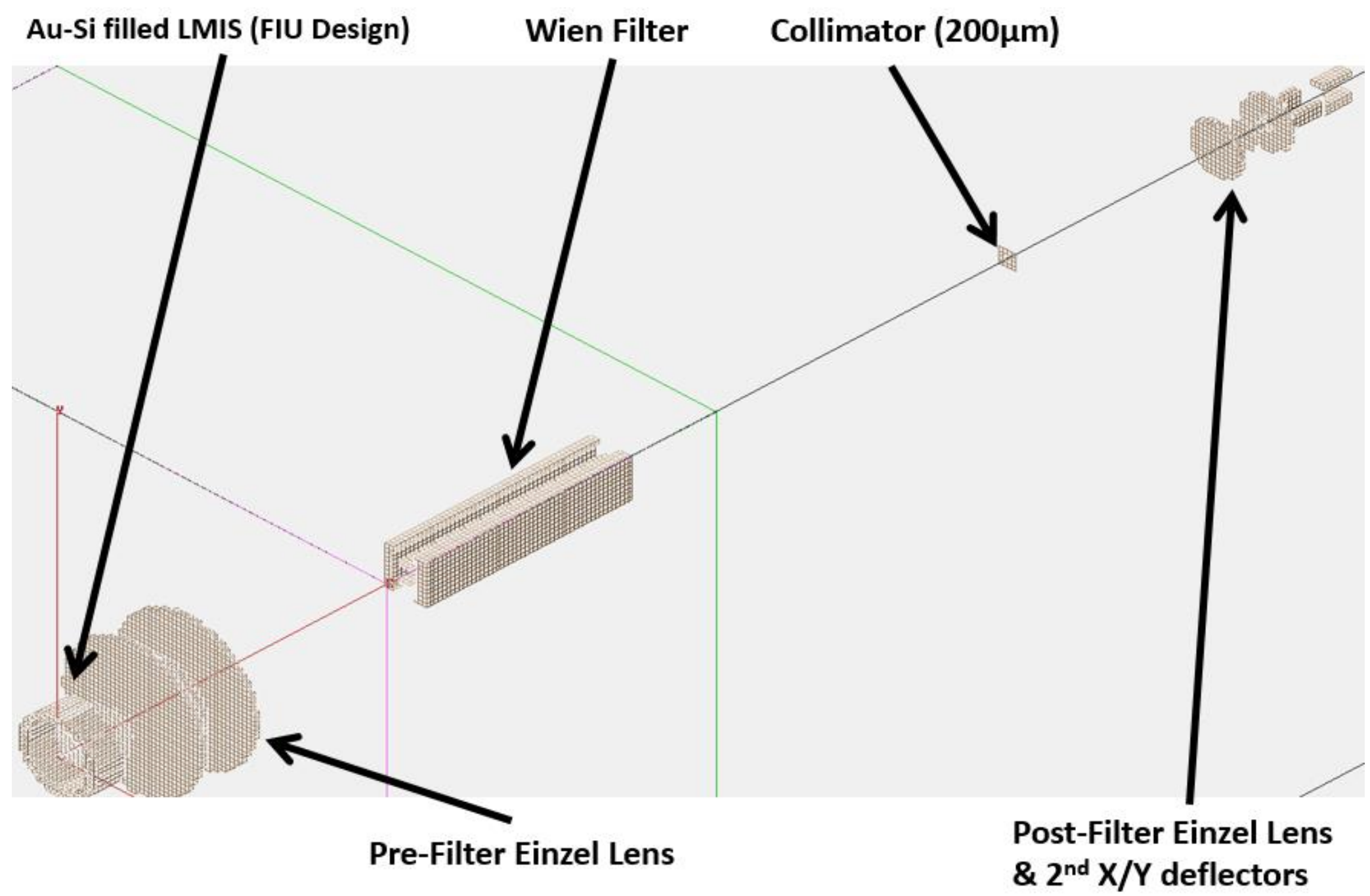

Figure 33. 3D view for ion optics simulation workbench for the theoretical evaluation of optics performance with components labeled. 


\subsubsection{Characterization of Gold Projectiles}

The characterization of gold projectiles from the Au-LMIS was performed in similar fashion to previous versions of the LMIS employing a Wien filter ${ }^{99-100}$. A fixed current of $2 \mathrm{~A}$ is expected to generate a magnetic field of roughly $0.175 \mathrm{~T}$. A projectile profile is obtained by sweeping the voltages across the Wien filter electrodes, starting from a max voltage of $200 \mathrm{~V}$, with fixed $1 \mathrm{~V}$ gradual stepwise decreases down to $0 \mathrm{~V}$. As the voltages are varied, the values for transmission of specific projectiles can be determined. This information can also be used to determine the effective mass resolution (separation) of the Wien Filter.

Current measurements of the beam constituents is performed on a faraday cup located $50 \mathrm{~mm}$ downstream from the collimator array, at the end of the PI column. However, feedthroughs are also arranged in a manner where current measurements can be performed on the collimator array by varying the aperture size. These measurements are mostly for evaluating beam stability and projectile-specific currents. Preliminary beam diameter measurements are performed utilizing a photoluminescent phosphorous plate, providing a light-emission based ability of measuring the emission volume. In combination with an optical feedthrough and a USB-based microscope camera, beam diameter and focusing conditions can be followed. 


\subsection{Results and Discussion}

\subsubsection{SIMION Theoretical Value Predictions}

The simulations for the generation of a high current-low focusing beam and a low current-high focus beam $(>10 \mu \mathrm{m})$ were completed by variations of the focusing optics potentials (Figure 35). $\mathrm{Au}_{400}{ }^{+4}$ projectiles had their energies calculated throughout flight, with the mean kinetic energy of the projectiles at the focal point having a mean value of $\sim 78.5 \mathrm{keV}$. This indicates a small energy spread from the original acceleration $(80 \mathrm{keV})$.
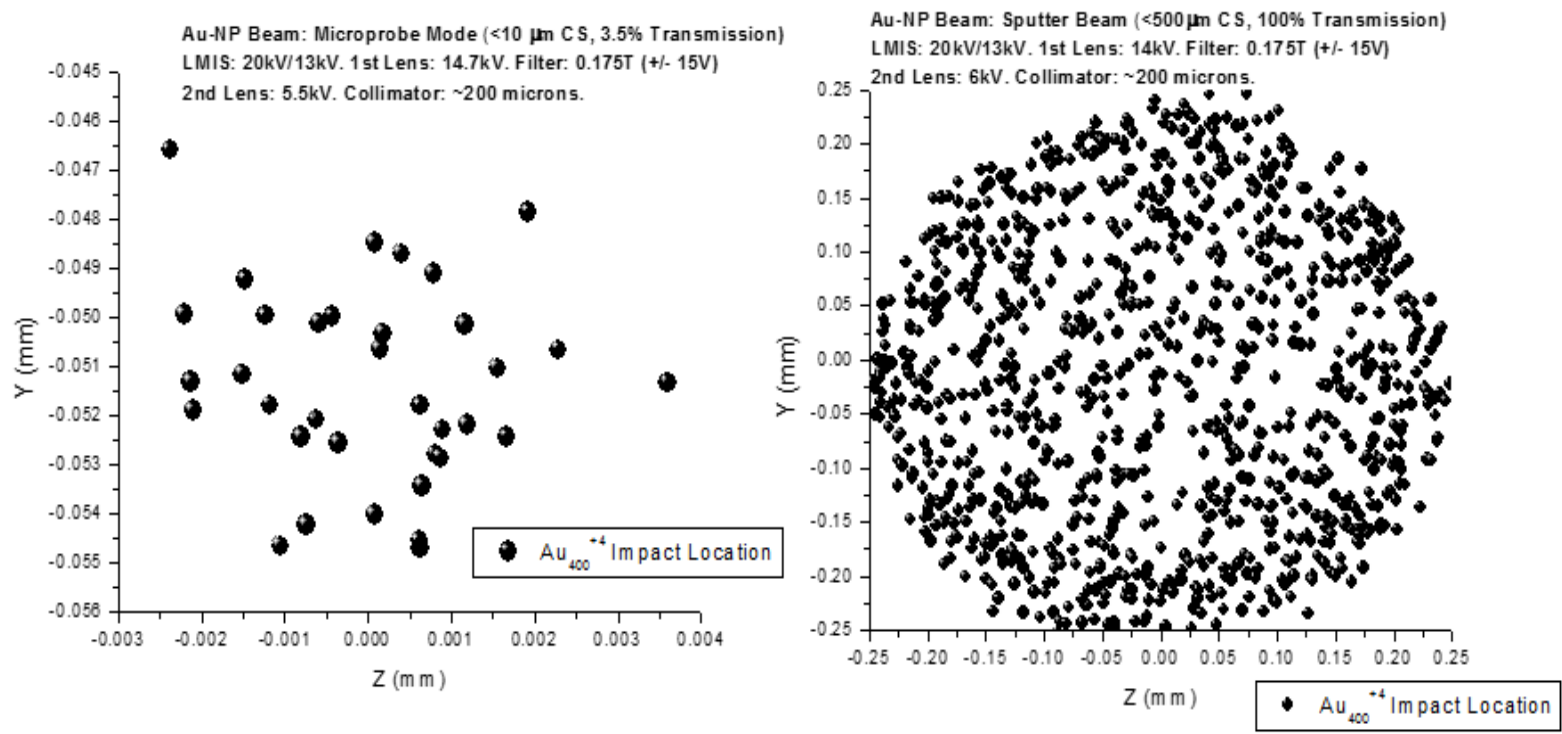

Figure 34. Au400+4 Beam cross-section profiles for low current-high focus (microprobe) and a high current-low focus (sputter) operational modes at a focal distance of $1173.6 \mathrm{~mm}$, the exit of the primary ion column.

Theoretical values for the generation of an Au-NP high-current sputter beam involved a pre-filter einzel lens voltage of $14 \mathrm{kV}$, with the collimator set to $200 \mu \mathrm{m}$, and a post-filter einzel lens voltage of $6 \mathrm{kV}$. These settings produced near $100 \%$ transmission effectiveness of Au-NPs, at the expense of low focusing. Figure 34 shows the beam cross-section profile at the focal point at the end of the PI column, where the spot size is $\sim 500 \mu \mathrm{m}$. This type of high fluence can be beneficial with the intent of operating the 
beam for dynamic SIMS application (sputter), or if looking into an event-by-event operation mode, which requires relatively high abundance of a particular projectile. Theoretical values were also found for the generation of an Au-NP low-current, highly focused beam for static SIMS applications. Pre-filter lens were set to $14.7 \mathrm{kV}$, shortening the focal point albeit achieving a greater reduction of aberrations around the axis of the beam. Post-filter lens were set to $5.5 \mathrm{kV}$, further focusing the transmitted ions for the remaining length of the ion optics workbench. The Wien filter potential for transmission of $\mathrm{Au}_{400}{ }^{+4}$ projectiles was kept at $15 \mathrm{~V}$, as this theoretically calculated value induced no deflection or diffraction to the beam and its trajectory. The overall settings for these simulations are illustrated in Figure 35. 


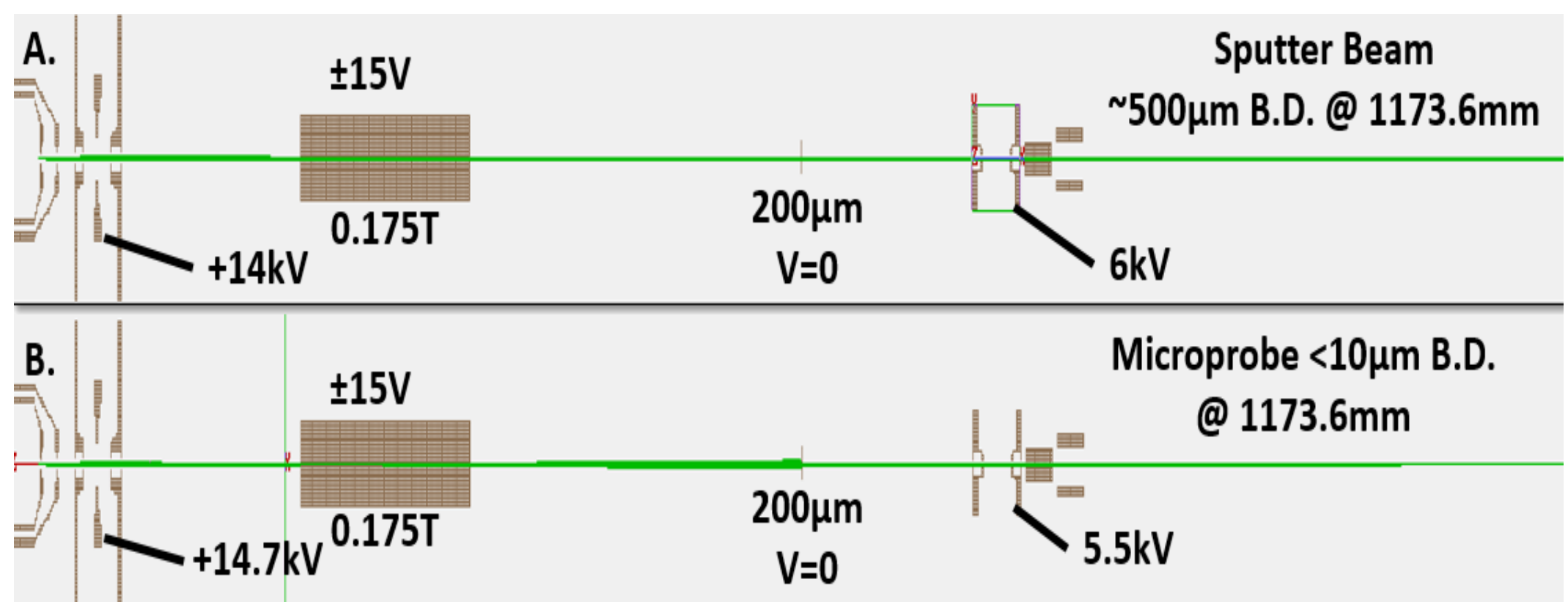

Figure 35. Simulations with optimized potential values for obtaining A) a high current sputter beam for dynamic SIMS applications and B) A low current, highly focused beam for nanoscale static SIMS. 


\subsubsection{Experimental Validation}

The source was set to a voltage of $20 \mathrm{kV}$, with the extractor voltage set to $13 \mathrm{kV}$, producing an extraction potential of $7 \mathrm{kV}$. Filament current was increased stepwise every 10min starting at $1 \mathrm{~A}$ and finishing at $2.6 \mathrm{~A}$, were the Au-Si eutectic had fully melted. Given a shortened focal length was desired for these experiments, the pre-filter einzel lens were given a potential value of $15.1 \mathrm{kV}$. Wien filter voltages were swept, while retaining fixed magnetic field of $\mathrm{B}=0.168 \mathrm{~T}$ ( $2 \mathrm{~A}$ on solenoids). The collimators were set to an aperture sizing of $500 \mu \mathrm{m}$. Measurements performed on the collimator surface as opposed to the Faraday cup at the end of column due to spacing constraints and discharging from the post-filter lens. Planning for overcoming these hurdles for future source characterization experiments has already taken place.

Starting from the lowest voltages, $\mathrm{Au}^{+}$projectiles were transmitted using electrode voltages from $178 \mathrm{~V}$ to $140 \mathrm{~V}$, with a maxima at $158 \mathrm{~V}$ for a current of $440 \mathrm{nA}$. Second projectile detected was the $\mathrm{Au}-\mathrm{Si}^{+}$projectiles, a common feature of utilizing $\mathrm{Au}-$ Si eutectic for an LMIS. This was followed by the transmission of $\mathrm{Au}_{2}{ }^{+}$projectiles using electrode voltages from $126 \mathrm{~V}$ to $104 \mathrm{~V}$, with a maxima at $116 \mathrm{~V}$ for a current of 58.54 nA. The third projectile transmitted was $\mathrm{Au}_{3}{ }^{+}$, with electrode voltages from $100 \mathrm{~V}$ to 80 $\mathrm{V}$, with a maxima at $92 \mathrm{~V}$ for a current of $35.65 \mathrm{nA}$. The fourth projectile transmitted was $\mathrm{Au}_{5}{ }^{+}$using electrode voltages from $72 \mathrm{~V}$ to $60 \mathrm{~V}$, with a maxima at $68 \mathrm{~V}$ for a current of $35.65 \mathrm{nA}$. Lastly, detection of the target projectiles $\left(\mathrm{Au}_{400}{ }^{+4}\right)$ was achieved with electrode voltages of $14.5 \mathrm{~V}$, with a current of $3.57 \mathrm{nA}$. These results fall in relative good agreement with the simulation values $(\mathrm{V}=14.79 \mathrm{~V})$. Results are shown in Figure 36. 


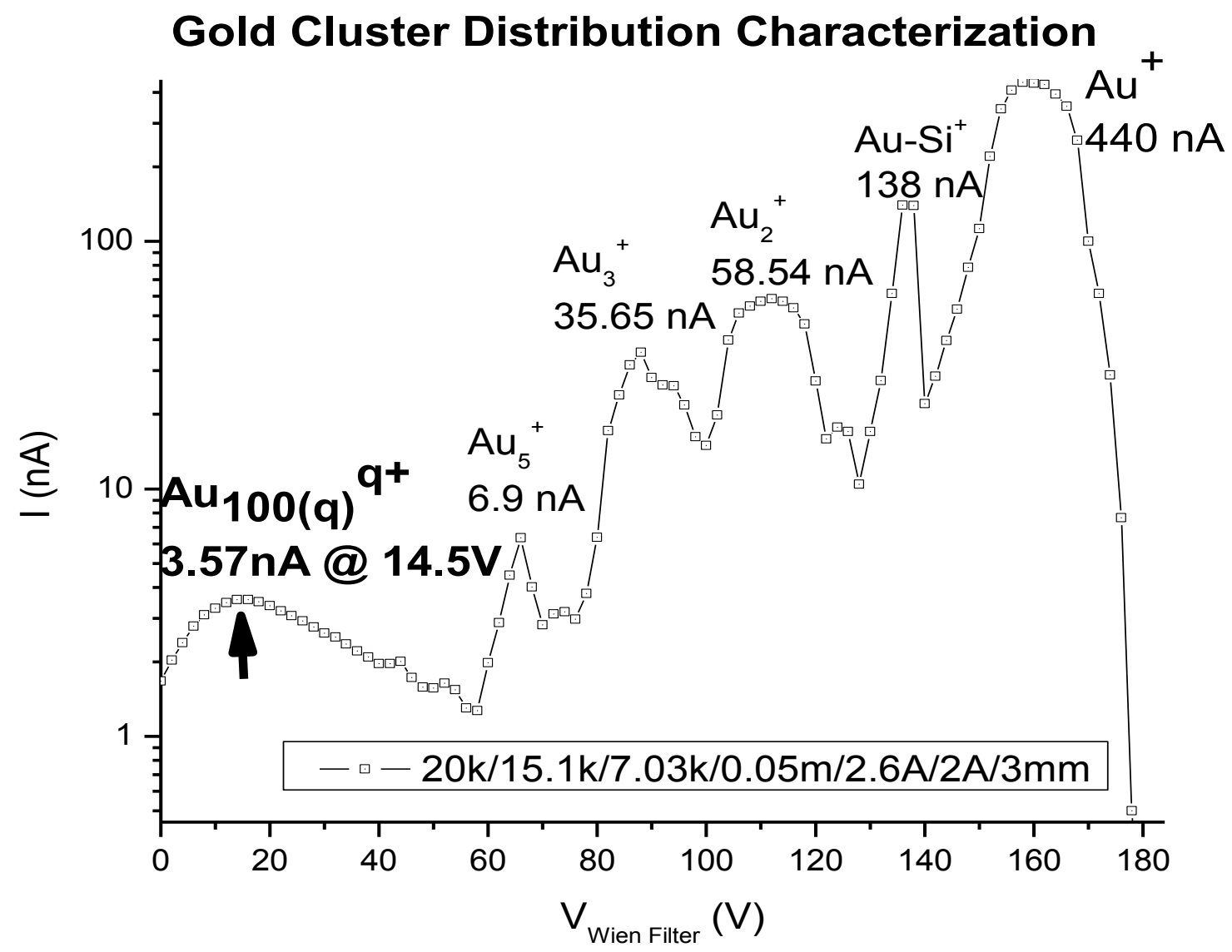

Figure 36. Results from Au-LMIS projectile characterization employing the Wien Filter with an emphasis of detection of massive gold clusters $\left(\mathrm{Au}_{400}{ }^{+4}\right)$.

Evaluation of the beam diameter showed, however a significant departure from simulation expectations. Figure 37 shows the results from an emission volume profiling experiment utilizing a phosphorous screen. Grids in the target sample are $1 \mathrm{~mm}^{2}$,

externally grounded to prevent rapid space charging. Lens were biased with respect to the simulation for obtaining the best possible beam diameter $(\mathrm{L} 1=14,750 \mathrm{~V}, \mathrm{~L} 2=5,500 \mathrm{~V})$ while the Wien Filter utilized the experimentally determined values. After correcting for side-emissions due to source wear, an experimental beam diameter of $750 \mu \mathrm{m}-1 \mathrm{~mm}$ was measured. 


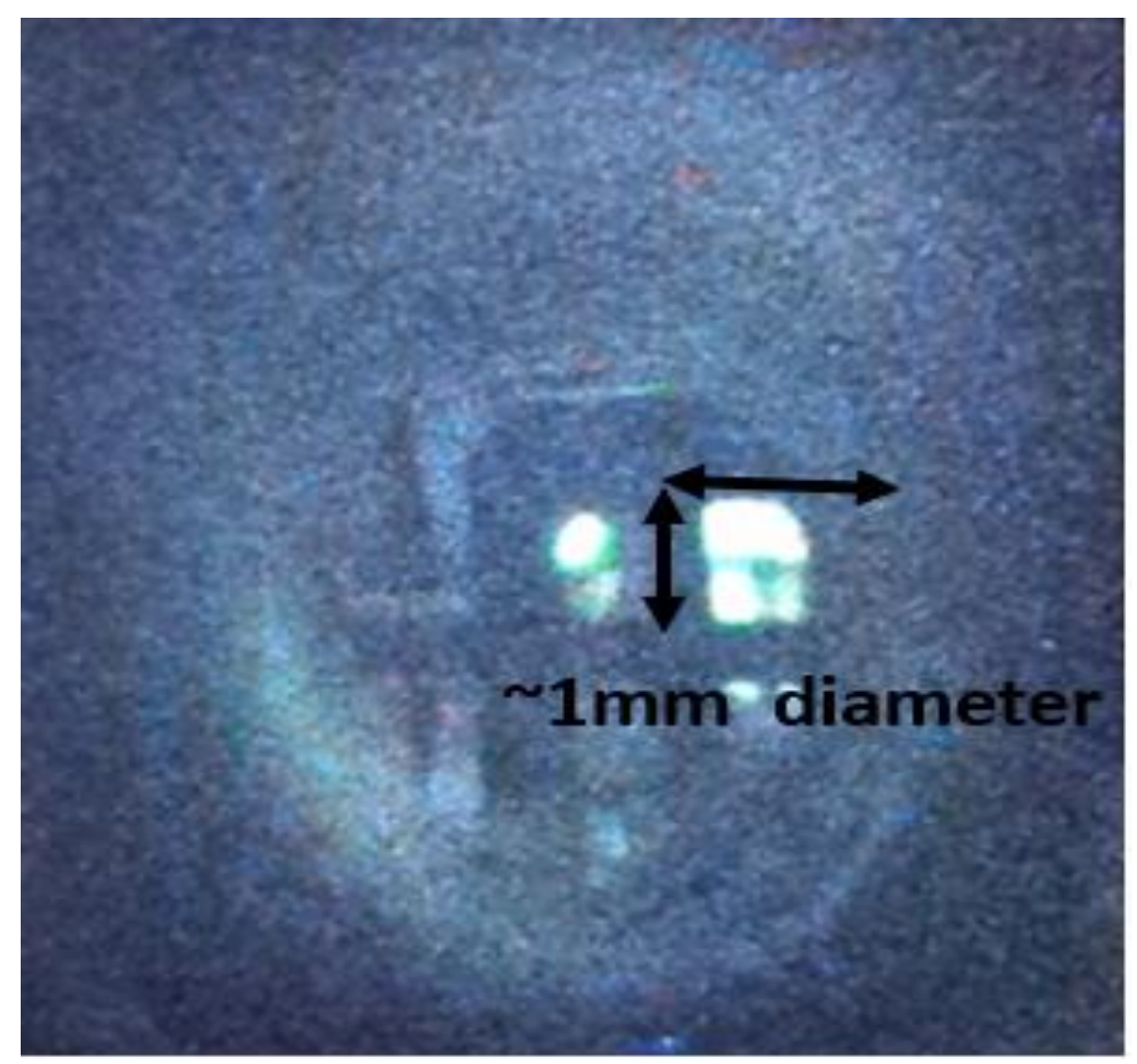

Figure 37. Camera view of bombarded phosphorous screen for beam diameter profiling. Focusing for the experiment above produced an $\mathrm{Au}_{400^{+4}}$ beam with a diameter of $\sim 1 \mathbf{~ m m}$.

\subsection{Conclusions}

An Au-NP Liquid Metal Ion Gun Source platform was created for the generation of massive gold cluster projectiles for surface analysis. The first step consisted of computer assisted design work for a LMIS Source, mounting/stage system, and electronics. Secondly, theoretical simulation combining the LMIS design and the PI column components for performance assessment was created. This simulation was utilized to accomplish a variety of tasks, including calculation of electric potential values for different operational conditions. Additionally, ion trajectories and energies are obtained as a function of flight distance and electrostatic fields. Thirdly, theoretical value predictions for the Wien filter's magnetic and electric fields for the transmission of key 
projectiles, including $\mathrm{Au}_{400}{ }^{+4}$, were made. Equipment for the fabrication and operation of the AuNP-LMIS sources were made, including vacuum emitter infusing chamber and specialized electronics for filament etching. Machining and fabrication of the source design and mounting system took place, with several modifications undertaken over time. Electronics for the operation of a $20 \mathrm{kV}$ LMIS were assembled. The Au-LMIS was attached to the PI column for experimental work and tested for stability. Beam characterization experiments were performed using the Wien Filter on the PI column, and a distribution of projectiles matching prior theoretical work was measured. The relative abundance of massive gold clusters was measured, with the recorded current and observed stability being satisfactory for their usage as a probe for the characterization of surfaces. 


\section{INTEGRATION OF THE PRIMARY ION COLUMN WITH AN FT-ICR MS.}

\subsection{Introduction}

Early in its inception, Secondary Ion Mass Spectrometry (SIMS) was limited to elemental and small molecule analysis ${ }^{11,19}$. Given only harsh, atomic probes were available, SIMS found itself with no usage in applications other than inorganic surfaces. However, this changed with pioneering work in the past 20 years, with projectile improvements opening the door for analysis of organic and biological surfaces analysis to become commonplace ${ }^{68,78}$. In the growing biological SIMS community, the general consensus is that the development of novel polyatomic and cluster probes has greatly enhanced the applicability of this technique to the study of cells for biomarker identification and localization ${ }^{3,11,15,19}$. These advances place SIMS in par with other surface analysis techniques (e.g., MALDI and DESI), with the advantage of exceptional spatial resolution and 3D chemical imaging capabilities ${ }^{9,3,15}$. Cluster projectiles like $\mathrm{Au}_{3}{ }^{+}, \mathrm{Bi}_{3}{ }^{+}$, and $\mathrm{C}_{60}{ }^{+}$, and massive projectiles like $\mathrm{Au}_{400}{ }^{+4}$, glycerol clusters, and $\mathrm{Ar}_{1500}{ }^{+}$ have provided enhanced secondary molecular ion yields, less fragmented species, and subsequently enhancing the effective mass range ${ }^{74-75,105,94,141}$. However, instrumentation limitations for these applications are not only limited to the ion probe, but also the mass analyzer. Most instrumentation for molecular SIMS analyses utilizes TOF or sector mass spectrometers, thanks to the compatibility with high vacuum, availability of positionsensitive multi-channel plates (MCP) for imaging, and wide dynamic mass range ${ }^{11,131}$. Notwithstanding, the limitations in terms of mass resolving power $\left(\mathrm{m} / \Delta \mathrm{m}_{50 \%}\right)$, measured mass accuracy, and lack of MS/MS capabilities make the accurate identification of molecular ions from biological samples a challenging endeavor ${ }^{15}$. In addition, the 
operation conditions for TOF-SIMS instruments may require a pulsed PI beam in conjunction with a pulsed extractor ${ }^{11,142}$. Such operation conditions impose a trade-off between mass resolution, mass range and spatial resolution, related on the pulse widths on the PI column and the SI extraction optics ${ }^{11}$.

In recent years, different instruments have been develop both in the research and commercial communities to meet these needs. The Winograd lab integrated an Ionoptika $\mathrm{C}_{60}$ PI column to an AB Sciex Q-Star MALDI-QqQ-o-TOF for analysis of biological targets, in particular for lipidomic studies and comparisons of performance with MALDI $^{143}$. Such an instrument is capable of MS/MS and MRM, operates at a mass resolution of mass resolving power $10,000-15,000$ at a $\mathrm{m} / \mathrm{z}$ of $400^{144}$. Issues regarding pressure gradients reduced the effective beam focusing, as well as transmission issues for SI transmission made MS/MS experiments problematic. IONOPTIKA, in collaboration with the Vickerman lab, released the J105 Chemical Imager ${ }^{145}$. This instrument generally employs a DC (dynamic) $\mathrm{C}_{60}{ }^{+}$beam, as opposed to conventional pulsed PI beam operation. By decoupling the PI beam operation from the mass analyzer, SIs can be subjected to MS/MS processes without affecting the PI source. Secondary ions are extraction through a series of lens and into a cooling cell prior to entering an electrostatic sector were transmitted ions have a narrow energy distribution. Here, ions arrive at a time-dependent buncher where ions retain their time focus and are then ejected simultaneously with a high-voltage pulse, followed by a collisional cell for MS/MS. Precursor/fragment ions are time-selected using an internal TOF gate prior to entrance to the main double-reflectron TOF (TOF-TOF) ${ }^{87}$. This instrument has been used for biological applications, with a spatial resolution of $\sim 200 \mathrm{~nm}(3 \mathrm{D} \text { imaging })^{146}$, a mass 
resolution of $\sim 10,000$ and $2 \mathrm{D} / 3 \mathrm{D}$ chemical imaging capabilities ${ }^{146,87}$. As of the time of this Thesis writing (Spring-Summer 2016), a powerful new option is being introduced into the commercial market ${ }^{147}$. Physical Electronics has recently completed the testing of a MS/MS (TOF-TOF-SIMS) instrument, capable of analyzing/imaging targeted father $\left(\mathrm{M}^{1}\right)$ and daughter ions $\left(\mathrm{M}^{2}\right)$ simultaneously ${ }^{147}$. Based on the patent description, this is done by extracting a packet of mass-selected SIs per PI pulse into a separate reflectron TOF, equipped with a high energy collisional cell for fragmentation prior to transmission. This ensures that meta-stable ions are not acting as isobaric/unresolvable interferences with respect to the target analyte ions (US Patent No. 20150090874 A1).

These instruments, while capable of providing MS/MS capability, and formidable mass accuracy, still lack the ability of properly resolving all species detected. These instruments also suffer from SI transmission issues when operating in MS/MS mode, requiring the beam to be continuously operated at a heavy dosage $\left(>10^{14}\right.$ ions $\left./ \mathrm{cm}^{2}\right)$ to obtain significant signal $^{15,143,145}$. This causes a rapid sputtering of the sample, requiring the usage of large cluster projectiles in order to avoid interlaying mixing. Nanometer resolution LMIG-based cluster sources like $\mathrm{Bi}_{3}{ }^{+}$are essentially barred from being utilized in this mode due to the potential for rapid damage accumulation otherwise unseen with $\mathrm{C}_{60}{ }^{+}$or larger projectiles ${ }^{11}$. Thin-layer samples such as novel organic materials (OLED’s) are incompatible with this mode of operation due to rapid sample depletion ${ }^{3}$. In order to overcome such challenges, the coupling of a cluster PI column source with an ultra-high resolution/sensitivity mass spectrometer such as an FT-ICR MS was explored ${ }^{89}$. Smith and cowokers at Pacific Northwestern Laboratory (PNNL) initially coupled an Ionoptika $\mathrm{C}_{60}{ }^{+}$source to a Bruker Daltonics SolariX 9T FT-ICR MS ${ }^{89}$. Being that SIMS is a high 
vacuum technique, this makes it incompatible with the stock electrodynamic extraction/focusing funnel of the SolariX. Custom housing for differential pumping and an RF octopole/quadrupole guide were added for the placement of the target plate and the transmission of SIs into the further parts of the instrument. Initial publications with this instrument operated the $\mathrm{C}_{60}{ }^{+}$source in a DC mode, where the SIs were continuously transmitted by the quadrupole ion guide into the first RF multipole of the SolariX, which is followed by a collision cell for MS/MS prior to FT-ICR MS analysis. Analysis of PEG (10000) and choleresterol on mice brain were accomplished with a minimal mass resolution of $100,000\left(\mathrm{~m} / \Delta \mathrm{m}_{50 \%}\right)$, sub-ppm mass accuracy, and a spatial resolution of $\sim 100 \mu \mathrm{m}^{89-90}$. MS/MS of the cholesterol from the mouse brain showed even higher resolution $(385,000)$, with clear identification of the generated fragments with respect to the parent ion. Last reported work with this instrument was performed by our research group, where it was utilized in combination with a dual-source TOFSIMS ${ }^{5}$ (IONTOF, Munster, Germany) instrument ${ }^{91}$. That is, a dual-instrument combination, where D. Discoideum cells were imaged with both instruments to evaluate inter-cell messaging mechanisms and to compare the performance in terms of spatial resolution and mass resolution of the FTICR-SIMS versus a commercial TOF-SIMS. The TOFSIMS ${ }^{5}$, while being capable of achieving high spatial resolution with $\mathrm{a} \mathrm{Bi}_{3}{ }^{+}$probe $(<1.5 \mu \mathrm{m})$, lacked the mass resolving power to properly characterize the chemical complexity of these cells and their messaging mechanism. The power of the FT-ICR MS instrument in combination with a $120 \mu \mathrm{m}^{2} \mathrm{C}_{60}{ }^{+}$beam in DC allowed for the identification of over 293 lipids, within a set criteria out of 2,595 peaks with a mean mass resolving power of $>150,000$ 
$\left(\mathrm{m} / \Delta \mathrm{m}_{50 \%}\right.$ at 150 to $\left.450 \mathrm{~m} / \mathrm{z}\right)$ and a mass accuracy of $5 \mathrm{ppm}$ or lower ${ }^{91}$. The superior mass resolving power is demonstrated in Figure 37.
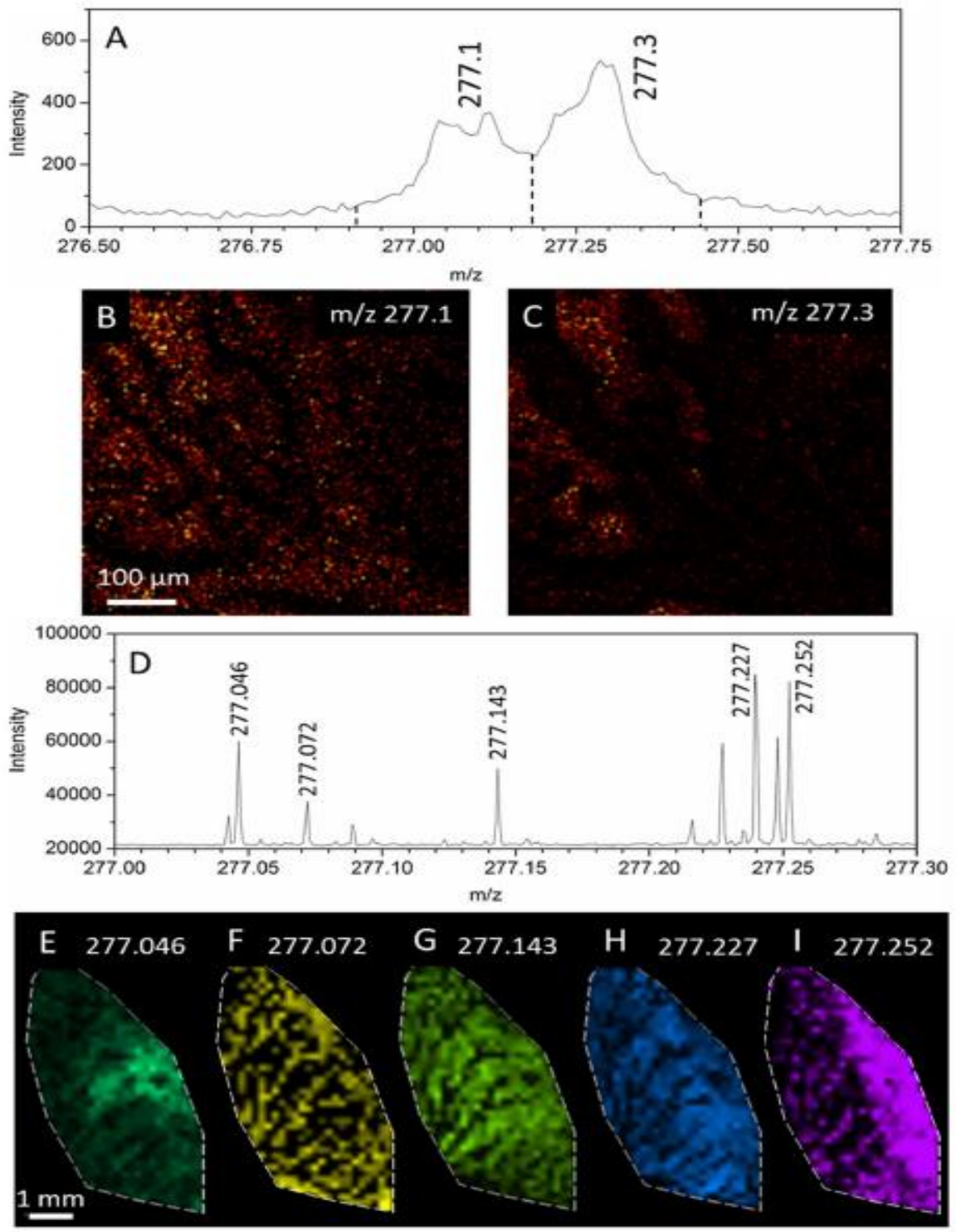

Figure 38. Reproduced with permission from DeBord, FFL, et al' $^{91}$. A),B), and C) show sub-micron spatial resolution imaging capabilities of the TOFSIMS5, yet the lack of resolving power makes chemical identification challenging at the selected $\mathrm{m} / \mathrm{z}$ $\left(\mathrm{m} / z\right.$ 277). D) and E) show the significant mass resolving power of the $\mathrm{C}_{60}{ }^{+}$FT-ICR MS instrument, with 5 characteristic peaks assigned within the $277 \mathrm{~m} / \mathrm{z}$ mass window. 
Having highlighted the power of FT-ICR MS for biological SIMS applications, we detail the plans for the coupling of an in-house custom-made PI column with a Bruker Daltonics SolariX 7T FT-ICR MS (Billerica, MA). A combination of ion dynamics simulations (SIMION 8.0), computer assisted design (CAD), and prototype component testing was employed to develop the interface between the PI column and the FT-ICR MS. Overall instrument assembly strives to direct the focused PI beam towards the SolariX housing (albeit some minor modifications), without the need for any extruding SI transfer optics. The currently proposed prototype involves the usage of a $90^{\circ}$ electrostatic sector for PI beam energy filtering and beam deflection towards the target plate ${ }^{134}$. The AuNP-LMIS $\left(\mathrm{Au}_{100(\mathrm{n})}{ }^{+\mathrm{qn}}\right)$ PI column can be utilized in DC and pulsed mode for SIMS analysis of organic surfaces. Au-NPs have been proven to be excellent as a PI thanks to their ability of generating intact molecular ions in large amounts and only having a damage-cross section of $\sim 10 \mathrm{~nm}^{101}$.

Having an electrostatic sector allows to further reduce chromatic aberrations by filtering ions with abnormal kinetic energies ${ }^{99}$. This is followed by a lens-extractor (Figure 15) for PI focusing. Rather than a direct annexation of the column to the FT-ICR MS housing, an orthogonal configuration is preferred to prevent beam distortion due to the SolariX 7 T magnet. Plans for extraction optics currently involve high-vacuum friendly, 8-element DC lens system for ion extration/focusing and subsequent injection the SolariX's first quadrupole ${ }^{130}$. In addition to developing the above described interface, a vacuum electrospray source for the preliminary evaluation of the new ion optic components was designed. As discussed in Chapter 1, several groups have been evaluating vacuum electrospray as a source for PI beams ${ }^{124,127}$. Glycerol and ionic 
liquids, polar and ionic in nature, have been proven to be suitable sources of ion beams with a suitable brightness. Here, we utilize an ionic liquid electrospray in vacuum to simulate the transmission efficiency of the lens utilizing the theoretically determined voltage values. This standalone test bench approach is intended to deliver faster turnaround times in evaluating theoretical performance with experimentally optimized values.

The lens system is also equipped with optical components such as a laser lens, a camera feedthroughs, and an LED for target visualization. These features allow us to not only have a powerful massive cluster SIMS instrument, but also take advantage of the SolariX's imaging and MS capabilities. This renders us with an ultra-high mass resolution (>100,000 m/ $\Delta \mathrm{m}_{50 \%}$ at $\left.400 \mathrm{~m} / z\right)$ and high-spatial resolution analytical tool ( $<10 \mu \mathrm{m})$, effectively capable of employing any cluster/massive cluster ion source compatible with the PI column's mounting system.

\subsection{Methodology for Interface Development and Testing}

As highlighted in the previous chapters, a combination of SIMION simulations and CAD software (Solidworks) was utilized to design and simulate the proposed optic components. Design primarily revolved around two main objectives, the delivery of the PI beam at the best possible spatial resolution while maintaining a significant ion count to the target at an incidence angle of $\sim 45^{\circ}$ with respect to the target. The second objective was the development of a robust system of optics for extraction and transmission of the SIs into the optics of the FT-ICR MS. These optics are to replace the two-stage electrodynamic funnel and should be able to extract ions on both negative and positive modes with high efficiency. Upon theoretical determination of optimized values fabrication, assembly, and preliminary experiments testing were 


\subsubsection{Design of the Interfacing Sector and Extraction Optics}

A $90^{\circ}$ electrostatic sector was designed to be placed after a gate valve, following the $2^{\text {nd }}$ einzel lens (Figure 39). The sector consists of two curved, 83.82 and $103.63 \mathrm{~mm}$ radius sections, respectively. The sections have a thickness of $9.52 \mathrm{~mm}$ and a spacing of $10.2 \mathrm{~mm}$ between them. This results in a mid-sector spacing of $\sim 93.5 \mathrm{~mm}$

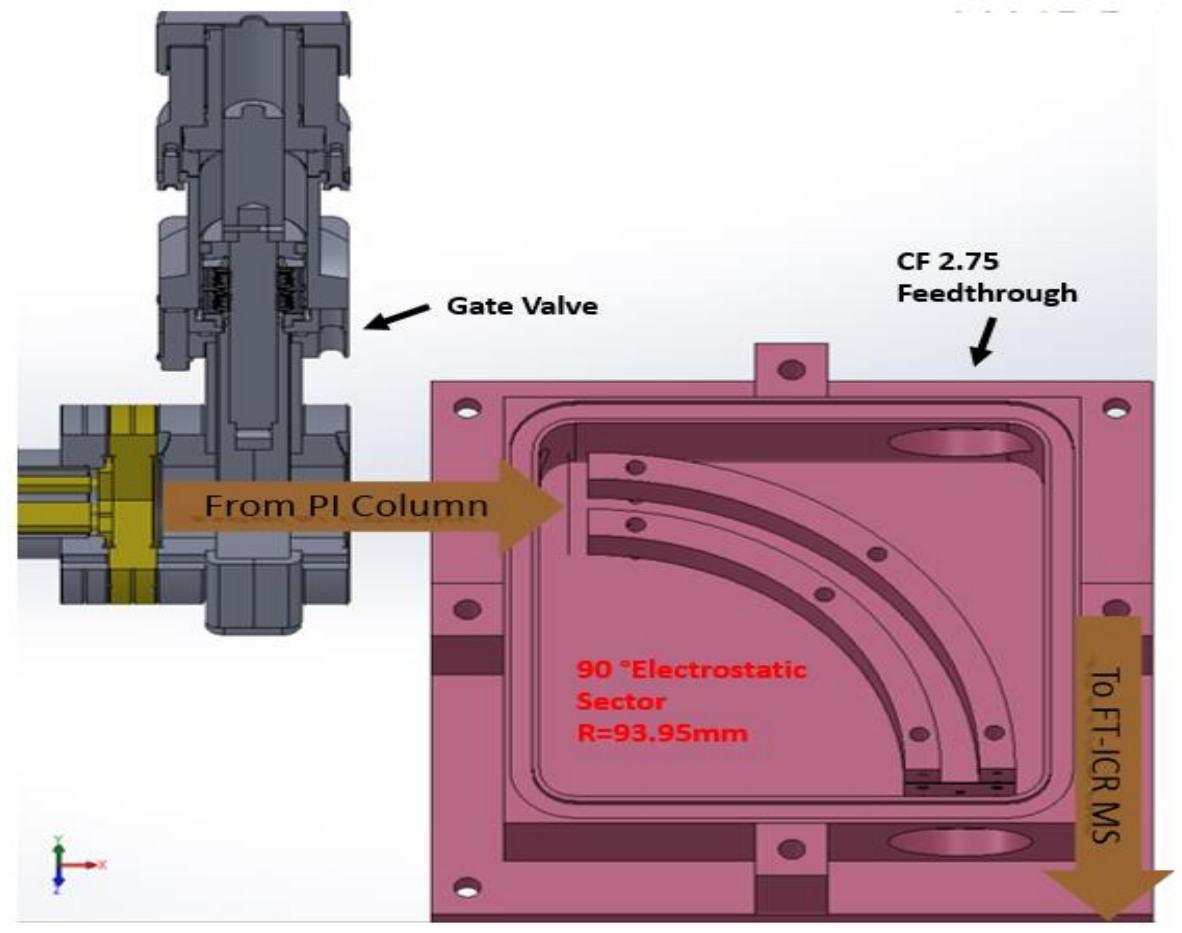

\section{Figure 39. 3D view of design for electrostatic sector to deflect the PI beam towards the FT-ICR MS.}

The electrostatic sector allows for energy-focusing of the PI beam, reducing chromatic aberrations. However, this is dependent on the energy spread of the incoming ion beam, as this will affect the current at the target. The chamber housing the sector has two CF275 flange mounts for MHV feedthroughs to bias the sector. At a distance of 55 $\mathrm{mm}$ from the sector, there is a third set of deflector plates, along with two grounding 
spacers for beam deflection. Since these deflector plates are incident to the target plate, they can be utilized for beam rastering (Figure 40).

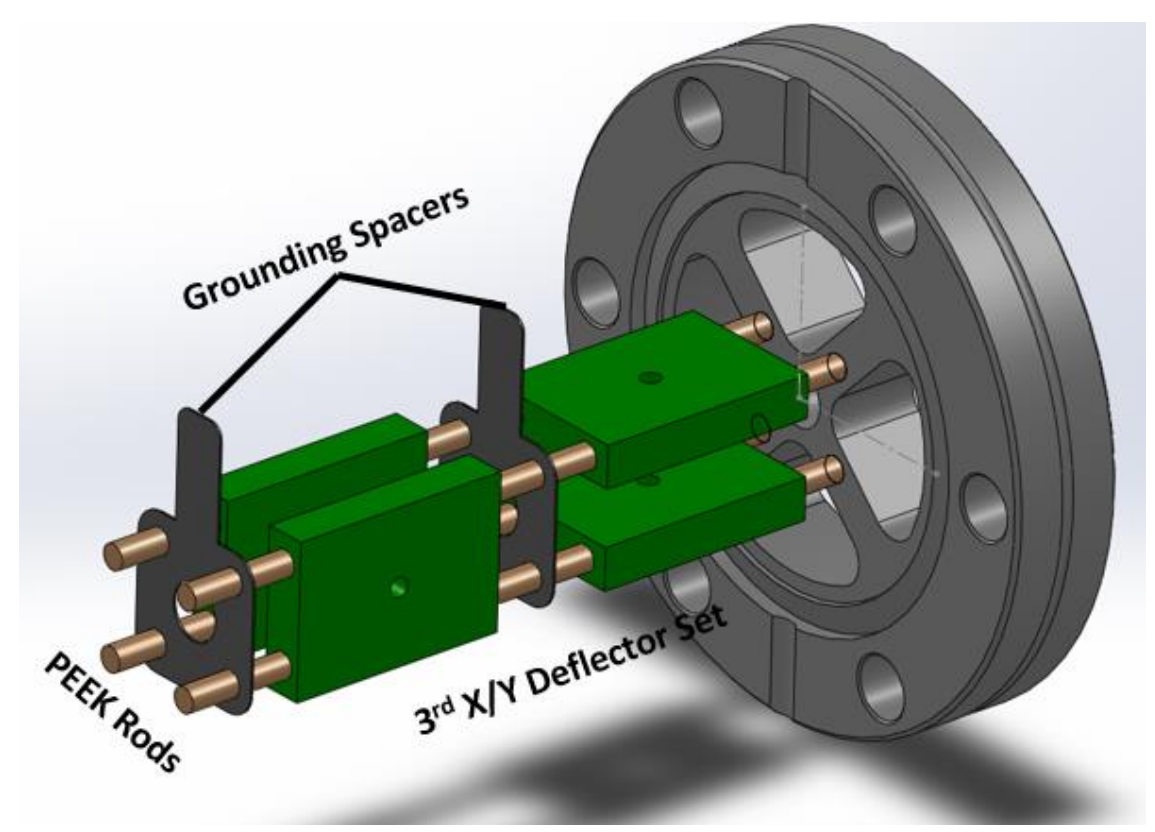

Figure 40. 3D view of primary ion beam rastering deflector plates with mounts for lens addition.

It must be noted that this system will be expanded to contain einzel lens similar to those presented in Figure 18. The plates are $25.54 \mathrm{~mm}$ x $17.46 \mathrm{~mm} \times 1.14 \mathrm{~mm}(\mathrm{~L} / \mathrm{W} / \mathrm{H})$, with $1.48 \mathrm{~mm}$ spacing between plates, and $12.95 \mathrm{~mm}$ space between plate pairs. The deflector flange is placed at the entrance of another in-house made chamber to give way for the incoming beam at a $45^{\circ}$ incidence angle. The chamber goes on top of the housing for the SolariX's ion funnel, replacing the stock roofing for this part of the instrument. The PI beam enters a final set of cylindrical lens of equal diameter, consisting of three electrodes with an outer diameter (O.D.) of $12.7 \mathrm{~mm}$, an inner diameter (I.D.) of 10.9 $\mathrm{mm}$, and $\sim 6.35 \mathrm{~mm}$ spacing. The first and second electrodes have lengths of $12.7 \mathrm{~mm}$ and $38.16 \mathrm{~mm}$, respectively. The third and final electrode is a cylinder electrode built on the SI extraction block, with a length of $12.7 \mathrm{~mm}$ with an internal diameter of $10.9 \mathrm{~mm}$. This 
geometry corresponds to an inner diameter/spacing ratio of 0.5 (D/f), correspondent to the lens strength with a focal length of $\sim 50 \mathrm{~mm}$.

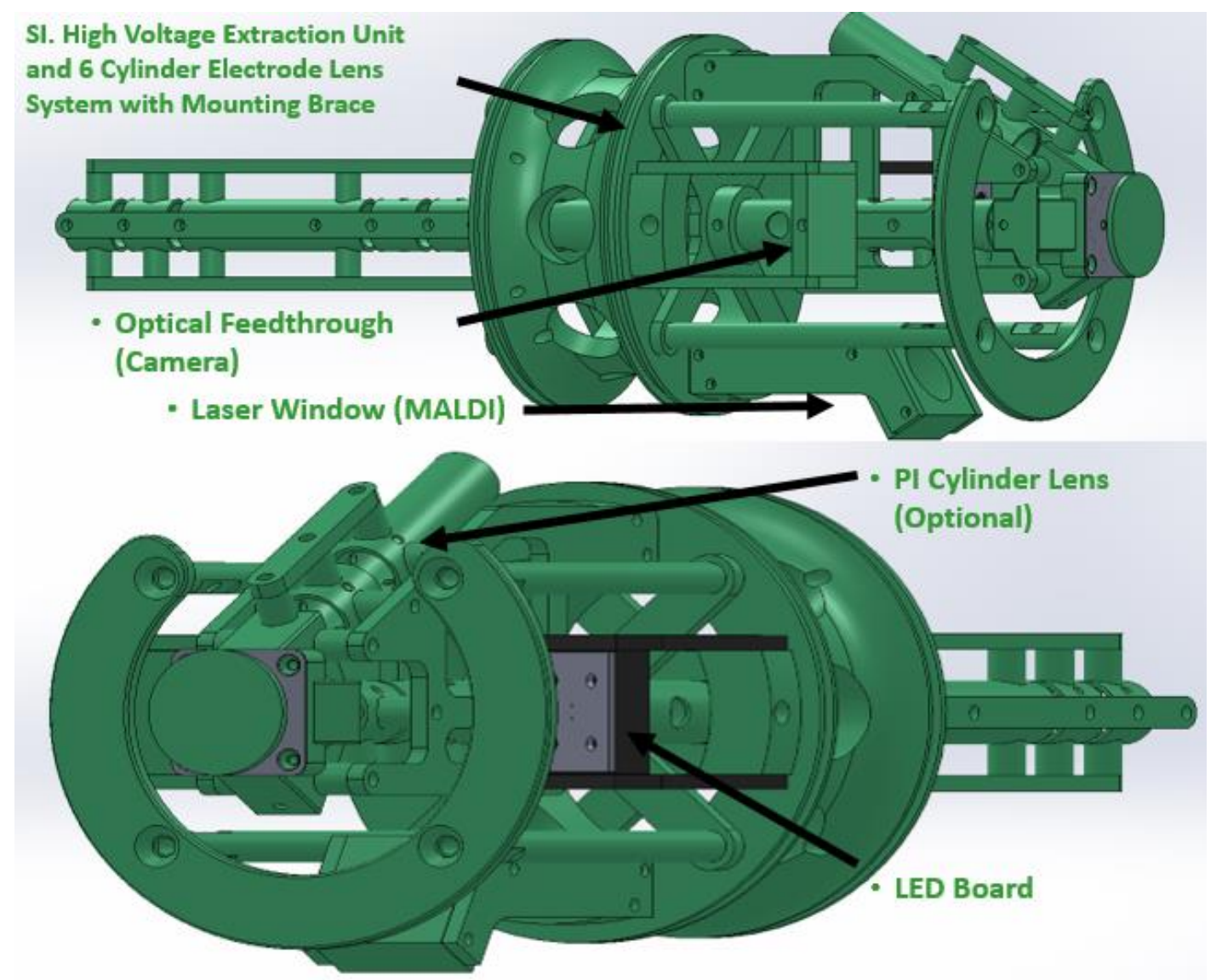

Figure 41. 3D view of secondary ion extraction lens system. Additional functionality features are highlighted.

From here the PI beam will impact the target plate, which is located 1-5 mm from a suppressor/extractor plate spot-welded onto the SI extraction block.

The suppressor/extractor plate is made of thin .316 stainless steel sheet metal plate and has a central pinhole of $2.38 \mathrm{~mm}$ for SI transmission and four other equidistant $2.38 \mathrm{~mm}$ apertures for an optical (camera) feedthrough, laser transmission, and for the illumination from the LED to reach the target plate. 
SI extraction starts with ions entering the extraction block through the central pinhole, and leaving through a dense electrode with a length of $12.7 \mathrm{~mm}$ and an inner diameter of $10.92 \mathrm{~mm}$ (cylinder electrodes built on the block are all symmetrical for field homogeneity). This SI transmission lens system consists of an additional 6 electrodes, all with the same thickness of $1.78 \mathrm{~mm}$ (12.7 mm O.D. and $10.92 \mathrm{~mm}$ I.D.), albeit of asymmetrical length and spacing. The first electrode, of $12.7 \mathrm{~mm}$ length/12.7 O.D./10.92 I.D. is positioned $1.59 \mathrm{~mm}$ away from the extraction block and the second electrode. The second electrode, positioned $3.17 \mathrm{~mm}$ from the third electrode has a length of $143.59 \mathrm{~mm}$ and is intended to act as the main SI focusing section. The third electrode is of $12.7 \mathrm{~mm}$ in length, and spaced $3.17 \mathrm{~mm}$ from both the second and fourth electrodes. The fourth electrode is $50.8 \mathrm{~mm}$, and spaced $3.17 \mathrm{~mm}$ from the third and firth electrodes. The final two electrodes, the 5th and 6th, are of $12.7 \mathrm{~mm}$ length and similar spacing, with the intention of operating as optional focusing elements.

Structural features involve a central, cylinder-like support made of Delrin similar to that of Bruker's stock ion funnel for fitting purposes. Support of the extraction block and other features is achieved by spacers and framing made of PEEK to prevent discharging. The first quadrupole inside of the SolariX has also been modeled, yet this component along with other features has been omitted from this thesis due to a confidentiality agreement. 
4.2.2 Development of a Total Simulation Involving Primary Ion Column, Interfacing Optics and Secondary Ion Extraction Optics

Development of the complete simulation started by first importing the interfacing optics with respect to their geometrical arrangement from the Solidworks design files. The sector and the lens were imported by converting the design files into stereolitography (.STL) files with a tolerance of $\pm 0.01 \%$ which were then imported into SIMION to output electrostatic physical arrays (.PA files). The PI column electrodes were rendered with a scaling of $0.1 \mathrm{~mm} / \mathrm{gu}(\mathrm{mm} / \mathrm{pixel})$ to produce a electrostatic field 10 times more refined than the baseline. Secondary ion optics were rendered with a scaling of $0.25 \mathrm{~mm} / \mathrm{gu}$ (mm/pixel), to produce a feature and subsequently electrostatic field resolution 4 times over the baseline. The physical arrays were then positioned with respect to the design drawing to accurately elucidate SI trajectories.

An individual simulation was created in for the evaluation of the SI extraction lens performance for transmitting ions into the first quadrupole of the SolariX FT-ICR MS, and transmission into the subsequent optics. The dimensions were provided by Bruker and a physical array (.PA\#) was made with this information (Figure 42). This simulation aimed primarily at observing the effects of SI trajectories through the lens and RFquadrupole guide. Figure 43 shows the total simulation created to evaluate overall performance of the instrument.

The PI column was tuned to achieve high current for DC operation and spatial focusing with the ultimate goal being a beam diameter of $<10 \mu \mathrm{m}$ and a transmission effective to hundreds of pA. Having measured $>3 \mathrm{nA}$ currents at the end of the column during the experimental phase, a transmission greater than $20 \%$ from the column into the 
target plate will translate to hundreds of picoamperes, enough to obtain a fluence of $1.0 \times 10^{14}$ ions $/ \mathrm{cm}^{2}$ for dynamic SIMS experiments. However, if there the energy-filtering effects of the electrostatic sector may significantly influence transmission towards the target plate. A wide energy spreads on the incoming ion will result in a lateral spread of our beam from the sector to the target plate, incurring undesired trajectories to some of the projectiles with the risk of being lost in flight. This stresses the importance of properly retuning our electrostatic lens, as improper tuning will result on a significant energy spread in the $\mathrm{Au}_{400}{ }^{+4}$ beam, increased beam lateral spread and subsequent projectile loss.

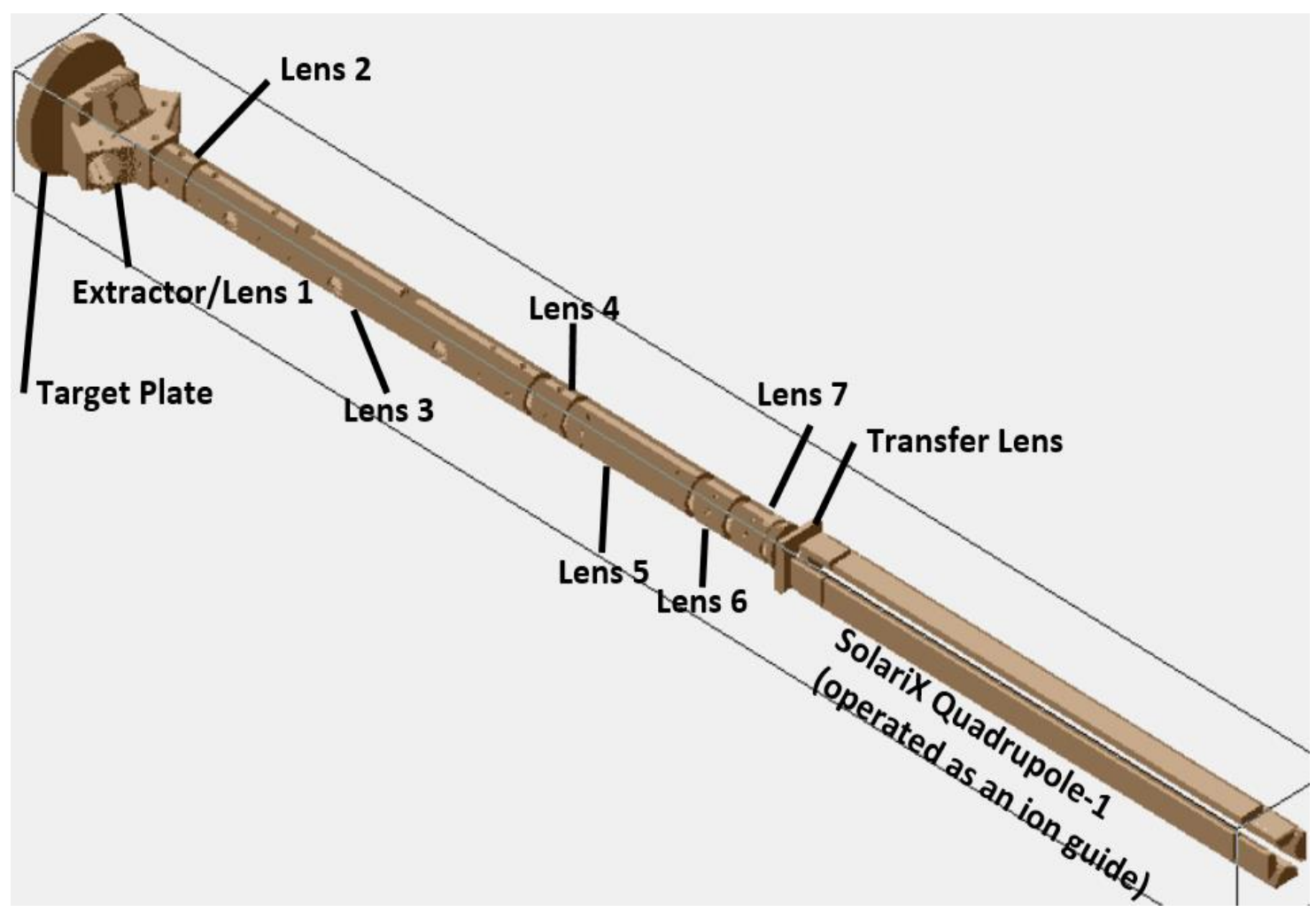

Figure 42. 3D isometric view of the simulation for evaluating secondary ion extraction lens performance and subsequent transmission into the SolariX's first component (Q1). 


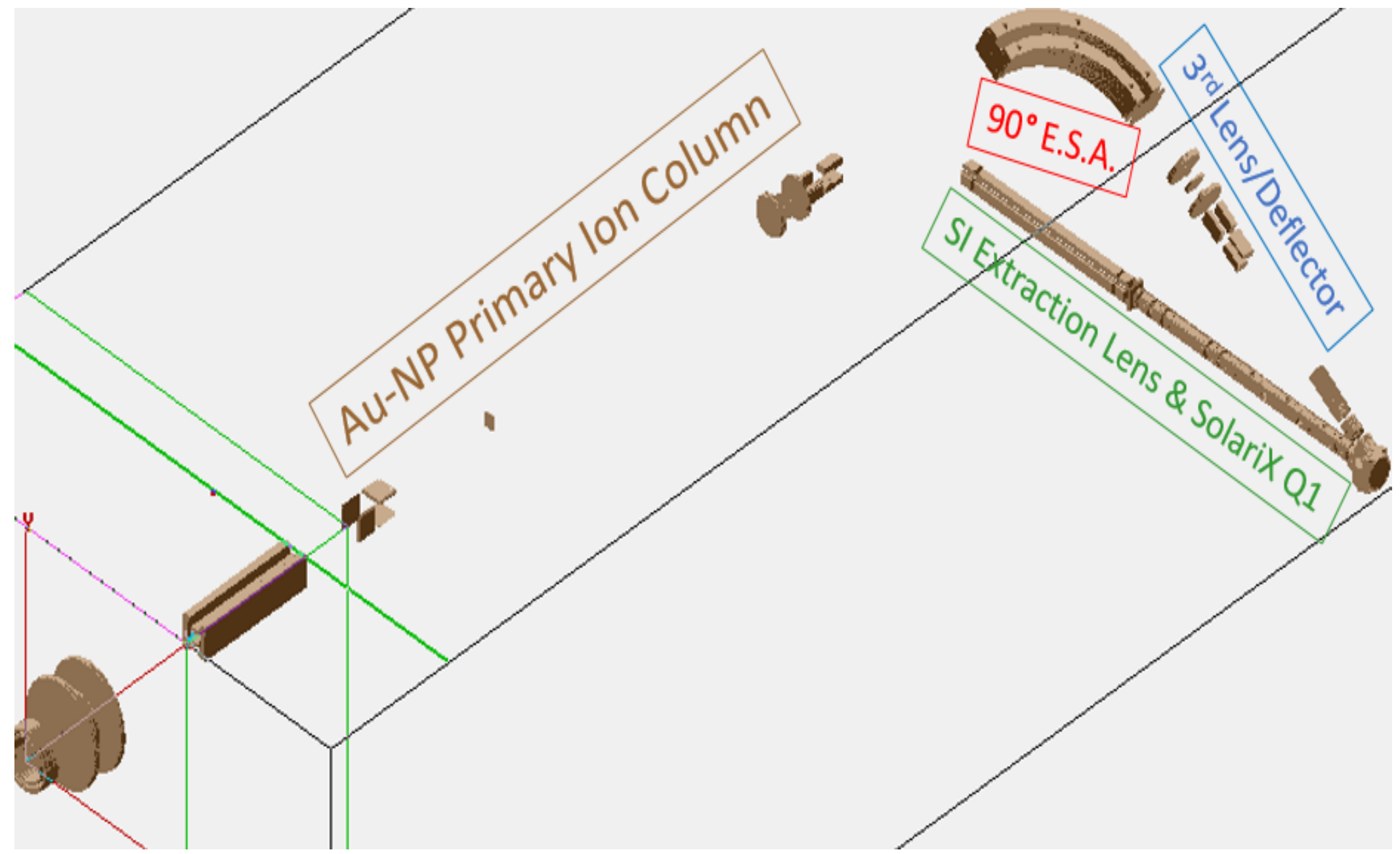

Figure 43. SIMION isometric view of total simulation with components labeled. 


\section{Primary Ion Column Optimization}

The distance of ion generation was chosen to reflect the spacing of nanodroplet formation in the Taylor cone with respect to the emitter. A wider mass and charge distribution of \pm 2 standard deviations was given to account for the different varieties of $\mathrm{Au}_{100(q)}{ }^{(\mathrm{q})+}$ projectiles ${ }^{99}$. Ions were given a more focused angle distribution, however with a kinetic energy spread of $0.1 \mathrm{eV} \pm 0.05 \mathrm{eV}^{99}$. These differences are better illustrated in Table 2. Under these conditions a recalculation of the voltages are required to achieve the best spatial resolution. PI column simulations first aimed to achieve the best focusing and PI transmission from the emitter from to the first lens ( $900 \mathrm{~mm}$ effective distance). This was done by evaluating beam diameter and PI transmission as a function of voltage starting at $13 \mathrm{kV}$ and finishing at $16 \mathrm{kV}$. Wien Filter values will be those acquired during experimentation. The original calculations inferred a fixed magnetic field (B) with a strength of $0.175 \mathrm{~T}$ at $2 \mathrm{~A}$ on the electromagnet, with an ion kinetic energy of $\sim 20 \mathrm{keV}$ assuming a singly charged projectile, and a fixed electrode distance of $0.0123 \mathrm{~m}$.

Preliminary experimental data with the Wien Filter for transmission of $\mathrm{Au}_{100 \mathrm{q}}{ }^{\mathrm{q+}}$ at a current of $2 \mathrm{~A}$ on the electromagnets occurred at a voltage of $\pm 14.5 \mathrm{~V}$ on the deflector plates, as opposed to the expected $15 \mathrm{~V}$. With this information we determined actual magnetic field by solving for expression (2.15).

$$
\begin{array}{r}
14.5 V=V_{A u_{400}^{4+}}(\text { Experimental }) \\
=B *(0.0123 m) * \sqrt{\frac{3.204353 \times 10^{-15} \mathrm{~J}}{2 *(196966.569 a m u)}} \\
B \pm=0.1684 T(\text { Experimental })
\end{array}
$$


Solving for the magnetic field gives us a field of $0.1684 \mathrm{~T}$, which corresponds to the lower electrode voltages than those expected. From here we focus on the optimization of the second lens, third lens, and electrostatic sector.

The second lens was optimized for transmission into the sector and onto the third lens while retaining or further reducing the beam diameter. Voltages utilized ranged from 3.75 $\mathrm{kV}$ to $7.5 \mathrm{kV}$ utilizing $500 \mathrm{~V}$ steps,

The electrostatic sector will not require thorough optimization, as it was designed to transmit ions within an energy window centered around $15-20 \mathrm{k}(\mathrm{q}) \mathrm{eV}$. Utilizing the radius dependent equation (4.2) for an electrostatic sector with a known predetermined radius $(\mathrm{r}, 93.5 \mathrm{~mm}$ or $0.935 \mathrm{~m})$ and energy $(\mathrm{E}=\sim 17.5 \mathrm{kqeV} \pm 2 \mathrm{keqV})$, a voltage range can be calculated.

$$
\begin{gathered}
r=\frac{2 \pm V_{\text {Sector }}}{E_{\text {ion }}} \\
0.0935 \mathrm{~mm}=\frac{2 \pm V_{\text {Sector }}}{17500} \\
V_{\text {Sector }}= \pm 1600-1610 \mathrm{~V}
\end{gathered}
$$

Within this specified voltage range, PI transmission should be possible, with minor optimization required. The fourth and final component of the optimization was lens 3 , which was optimized in similar fashion to lens 2 . The optimization of the second and third lens is of importance, as these effectively determine the spatial resolution at the target. All these conditions are summarized in Table 3. 


\section{Secondary Ion Extraction Cartridge}

For the SI lens evaluation, ion emission initial conditions were modeled for an unfocused beam with $\sim 500 \mu \mathrm{m}$ offset emission (assuming a focused, high fluence beam) and a subsequent $30^{\circ}$ half-angle conical distribution for SI trajectories (see Table 4). SIs with internal kinetic energy of $0.01 \mathrm{eV}$ and a charge value of $1(\mathrm{q}=1)$ were used. Ions were given an overall mass range of $150-1100 \mathrm{~m} / \mathrm{z}$, divided into three distributions detailed in Table 4. Parameters of interest were SI transmission efficiency and kinetic energy spread. The first quadrupole inside the SolariX was modeled to operate as an ion guide with a fixed frequency of $880 \mathrm{kHz}$, no DC voltages on the poles, and employing the $\mathrm{RF}$ amplitude as an SI low mass cut-off tool. Simulations were performed utilizing a target plate-extractor distance of $5 \mathrm{~mm}$, although the values for the extractor are valid regardless of the distance. The target plate can be biased accordingly to propel SIs towards the extractor. 


\begin{tabular}{|c|c|c|c|c|c|c|}
\hline & \# of lons & Mass (amu) & Charge $(q+)$ & Ion Generation Position(X/Y/Z) & $\begin{array}{l}\text { Velocity Format } \\
\text { (Direction Spread) }\end{array}$ & $\begin{array}{l}\text { Initial } \\
\text { Energy }\end{array}$ \\
\hline Old & $\begin{array}{l}1000 \text { per } \\
\text { simulation }\end{array}$ & $\begin{array}{l}78786.628 \pm 1970 \\
\text { (Gaussian) }\end{array}$ & 4 & $\begin{array}{l} \pm 25 \mathrm{mrad} \text { circle distribution from } \\
\text { point of emission from emitter }\end{array}$ & $\begin{array}{l}70^{\circ} \text { Azimuth/Elevation } \\
\text { Circle Spread }\end{array}$ & $0.1 \mathrm{eV}$ \\
\hline New & $\begin{array}{l}10000 \text { per } \\
\text { simulation }\end{array}$ & $\begin{array}{l}78786.629 \pm 1970 \\
\text { (Gaussian) }\end{array}$ & $\begin{array}{c}4 \pm 2 \\
\text { (Gaussian) }\end{array}$ & $\begin{array}{l}\text { Single Point Emission, } 10 \mu \mathrm{m} \text { away } \\
\text { from the emitter }\end{array}$ & $\begin{array}{l}90^{\circ} \text { Cone Spread (w/ } \\
\text { respect to axis) }\end{array}$ & $\begin{array}{r}0.1 \pm 0.05 e V \\
\text { (Gaussian) }\end{array}$ \\
\hline
\end{tabular}

Table 2. Comparison of primary ion generation conditions between initial simulations and final simulations.

\begin{tabular}{|c|c|c|c|c|c|}
\hline $\begin{array}{c}\text { Source } \\
\text { Voltage }\end{array}$ & $\begin{array}{c}\text { Primary Lens } \\
\text { Voltages }\end{array}$ & $\begin{array}{c}\text { Second Lens } \\
\text { Voltages }\end{array}$ & Sector Voltage & $\begin{array}{c}\text { Third Lens } \\
\text { Voltages }\end{array}$ & SI Extraction Block \\
\hline \multirow{2}{*}{$20,000 \mathrm{~V}$} & $\begin{array}{c}13000-16000 \mathrm{~V} \\
(250 \mathrm{~V} \text { steps })\end{array}$ & $\begin{array}{c}3750-7500 \mathrm{~V} \\
\text { (500V Steps) }\end{array}$ & $\begin{array}{c} \pm 1600-1610 \mathrm{~V}, \text { modified based on } \\
\text { Transmission }\end{array}$ & $\begin{array}{c}3750-7500 \mathrm{~V} \\
\text { (500V Steps) }\end{array}$ & $\begin{array}{c} \pm 2100 \mathrm{~V} \text { depending } \\
\text { on + or - mode }\end{array}$ \\
& & & & \\
\hline
\end{tabular}

Table 3. Workflow for the optimization of the primary ion column and interface optics. Voltages are swept stepwise within the established range to maximize a) primary ion transmission as a function of b) primary ion focus.

\begin{tabular}{|c|c|c|c|c|c|}
\hline$\#$ of lons & Mass (amu) & Charge & Source Position(X/Y/Z) & Velocity Format (Direction + Kinetic Energy) & Color \\
\hline \multirow{3}{*}{10000} & $225 \pm 75$ & \multirow{3}{*}{1} & $X=-307.1$ (Single Point) & \multirow{3}{*}{90 Cone Distribution/0.1eV $\pm 0.05 \mathrm{eV}$ Initial Kinetic Energy Distribution } & Blue \\
\hline & $500 \pm 250$ & & $Y=20.5-20 \mathrm{~mm}\left(\sim 500 \mathrm{um}^{2}\right.$ Volume $)$ & & Red \\
\hline & $700 \pm 300$ & & $Z=-21.25-21.75$ ( 500um ${ }^{2}$ Volume) & & Green \\
\hline
\end{tabular}

Table 4. Simulation parameters for evaluating secondary ion extraction lens performance 10,000 projectiles of three different $\mathrm{m} / \mathrm{z}$ ranges $(150-300 \mathrm{~m} / \mathrm{z}, 250-750 \mathrm{~m} / \mathrm{z}, 400-1000 \mathrm{~m} / \mathrm{z}$ ) for 30,000 in total were flown in a simulation accounting for Coulombic repulsion and an initial energy of $0.1 \mathrm{Ev} \pm 0.05 \mathrm{Ev}$ to accurately represent cluster-projectile generated secondary ion trajectories. 


\subsubsection{Experimental Evaluation of Optics}

Following the theoretical determination of optimal voltage values for the lens a test bench was designed. The components utilized in this test bench are shown in Figure 44. The goal of the test bench is to measure the current of ions transmitted through the lens as a function of the voltage values on each of the electrodes. In order to apply the electrode voltage, a resistor chain was fabricated. Calculation of the required resistor values to obtain the desired voltage on each electrode is defined as follows:

$V(\text { initial })_{L 1} \stackrel{I * R 1=\Delta V_{1}}{\longrightarrow} V_{L 2} \stackrel{I * R 2=\Delta V_{2}}{\longrightarrow} V_{L 3} \stackrel{I * R 3=\Delta V_{3}}{\longrightarrow} V_{L 4} \stackrel{I * R 4=\Delta V_{4}}{\longrightarrow} V_{L 5} \stackrel{I * R 5=\Delta V_{5}}{\longrightarrow} V_{L 6} \stackrel{I * R 6=\Delta 6}{\longrightarrow} V_{L 7}=0$

For which the total summed resistor values should yield a net voltage of 0 at the last electrode defined as

$$
V_{\text {Final }}=0=I R_{\text {total }}=\sum R_{1}+R_{2}+R_{3} \ldots R_{N} ; N=6, \mathrm{I}=1 \mathrm{~mA}
$$

expanding this expression for the calculation of resistor values based on voltage differences amongst electrodes with a fixed current, yields

$$
\begin{gathered}
V_{\text {Final }}=0=I R_{\text {total }} \\
=\sum\left(R_{1}=\frac{\Delta V_{1}}{I}\right)+\left(R_{2}=\frac{\Delta V_{2}}{I}\right)+\left(R_{3}=\frac{\Delta V_{3}}{I}\right)+\left(R_{4}=\frac{\Delta V_{4}}{I}\right)+\left(R_{5}=\frac{\Delta V_{5}}{I}\right)+\left(R_{6}=\frac{\Delta V_{6}}{I}\right)
\end{gathered}
$$

After obtaining the theoretical voltages from simulations, these can be input into the above expression to calculate the values for the individual resistors. 


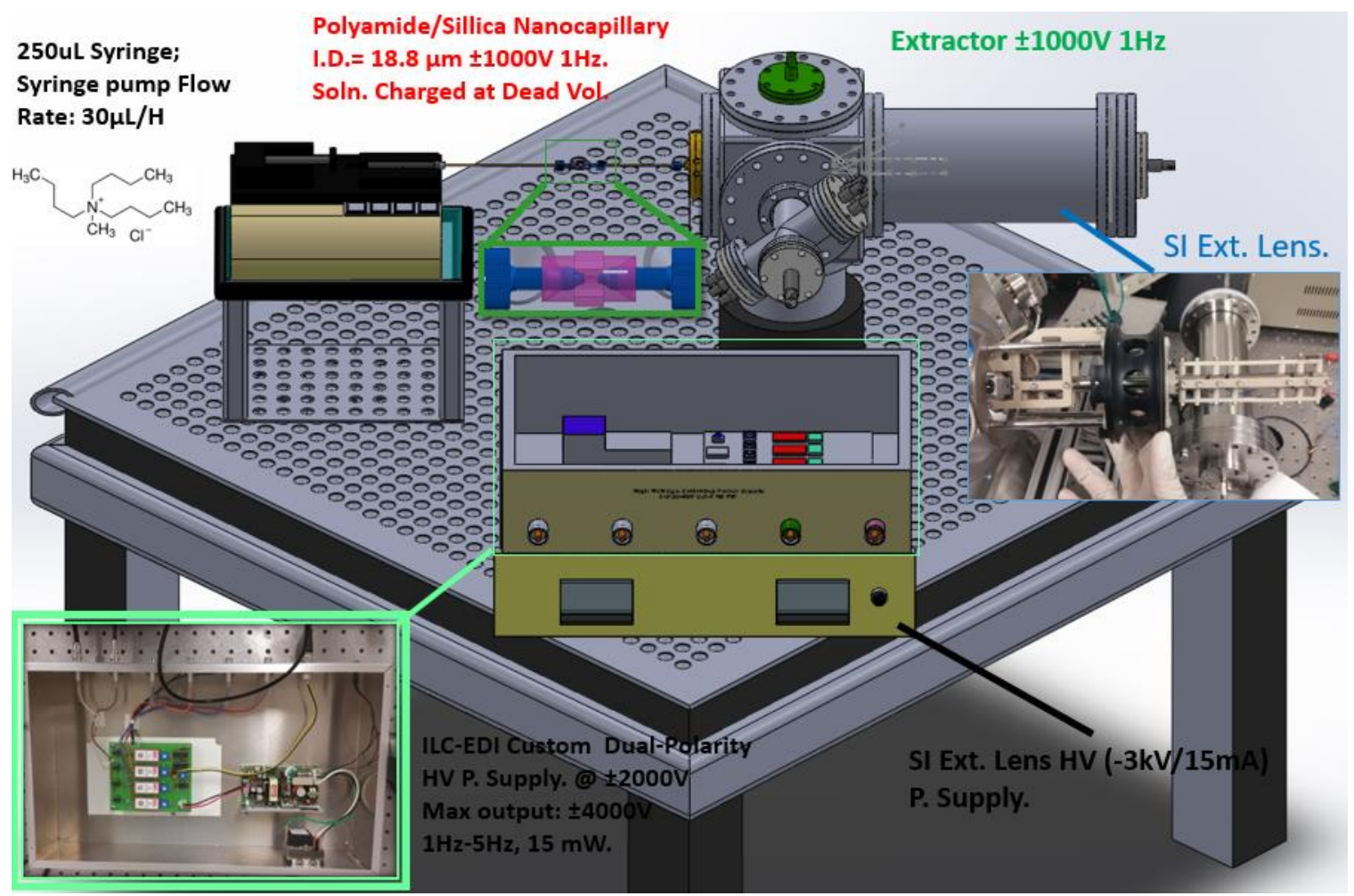

Figure 44. Design for standalone secondary ion extraction lens test bench with components highlighted and labeled. Insert show the specialized electronics utilized to generate a stable ionic liquid beam and the assembled secondary ion extraction lens.

\subsection{Results}

Results for this work have been divided into two steps following the design of the interfacing optics and related components. The initial step is the theoretical evaluation and optimization of instrumental parameters to assess performance. This consisted of accessing the best PI column conditions to transmit an $\mathrm{Au}_{400}{ }^{+4}$ beam with the best fluence/beam diameter ratio. The next step was to evaluate the performance of the SI extraction lens individually to assess SI transmission into the SolariX, output energy, and subsequent transmission into the first quadrupole of the FT-ICR-MS optics. The following step was the fabrication and assembly of the interface component parts, as well 
as the vacuum electrospray source for individual interface optic evaluation. This was finally followed by stand-alone performance evaluation of simulated transmission of secondary ions on the extraction lens cartridge as a function of voltage.

4.3.1 Theoretical Optimization of Primary Ion Column with Interfacing Optics and Secondary Ion Extraction Optics

Initial simulation results with the first lens displayed a uniform distribution of focusing as a function of transmission. Utilizing the initial conditions stated in Table 2-4, the best voltage was found to be $14 \mathrm{kV}$. Results are shown in Figure 45, where ion beam diameter and transmission was measured as the entrance of the second lens, or a distance of $920 \mathrm{~mm}$ from the source. Operating the first lens at $14 \mathrm{kV}$ gave a transmission of $\sim 30 \%$ with a beam diameter of roughly $\sim 1 \mathrm{~mm}$. This corresponds more properly to the experimentally observed beam behavior described in Chapter 3. While lower voltages offer greater fluences, these exhibit an axial spread making them unsuitable for maximizing spatial resolution. It must be highlighted that the Wien Filter was operated on the experimentally derived conditions, with a magnetic field (B) of $0.168 \mathrm{~T}$ and electrode voltages of $\pm 14.5 \mathrm{~V}$. This, combined with the wider mass and charge distributions account for the low \% transmission, as a large amount of the beam is lost within the Wien Filter or $200 \mu \mathrm{m}$ collimator from filter-induced trajectory deflection. Having determined the first lens value, the focus turns to the evaluation of lens 2 and 3 . As mentioned, the sector was operated at a range of 1600-1610 V during simulations to ensure no severe deflection of the beam occurs during transit through the sector or upon exit. 


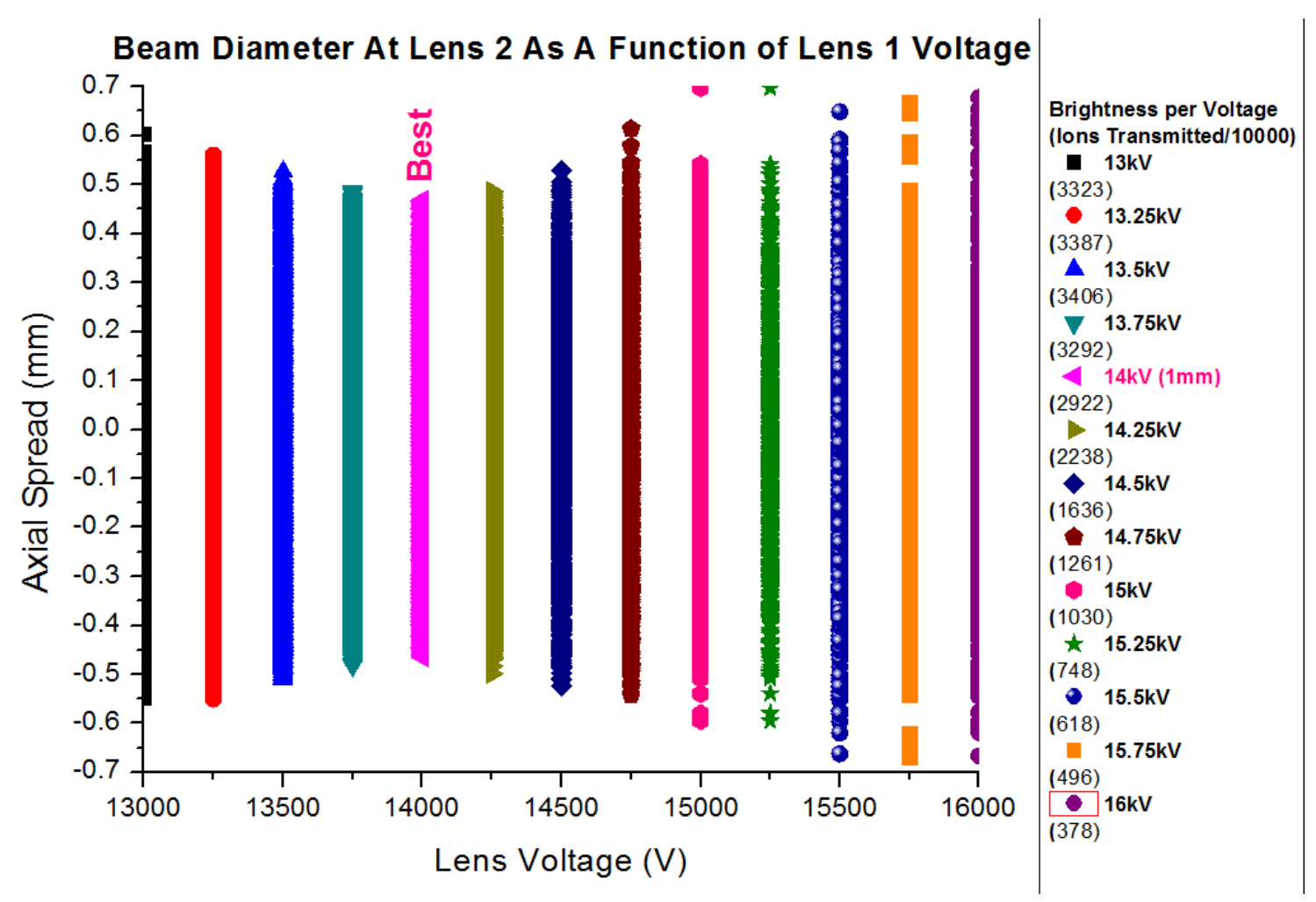

Figure 45. Results from the profiling of primary ion beam focus and transmission after velocity-selection and collimation.

After evaluating lens 2 and 3, it was found operation is best when given equal potentials. All other simulation results are omitted as the results are mostly unsatisfactory (beam diameters in excess of $500 \mu \mathrm{m}$ ). This may be due to the fact they have the same geometry, thus achieving the best focal distance when operated in equivalent fashion. Simulations to assess the best beam diameter were then performed utilizing symmetrical voltages for ion beams on negative and positive ion modes. Polarity-specific performance was assessed by the voltages on the SI extraction unit $+2.1 \mathrm{kV}$ for negative mode and -2.1 $\mathrm{kV}$ for positive mode, respectively. Figure 46 and Figure 47 illustrate the results of the negative and positive ion mode beam profiles, respectively. 


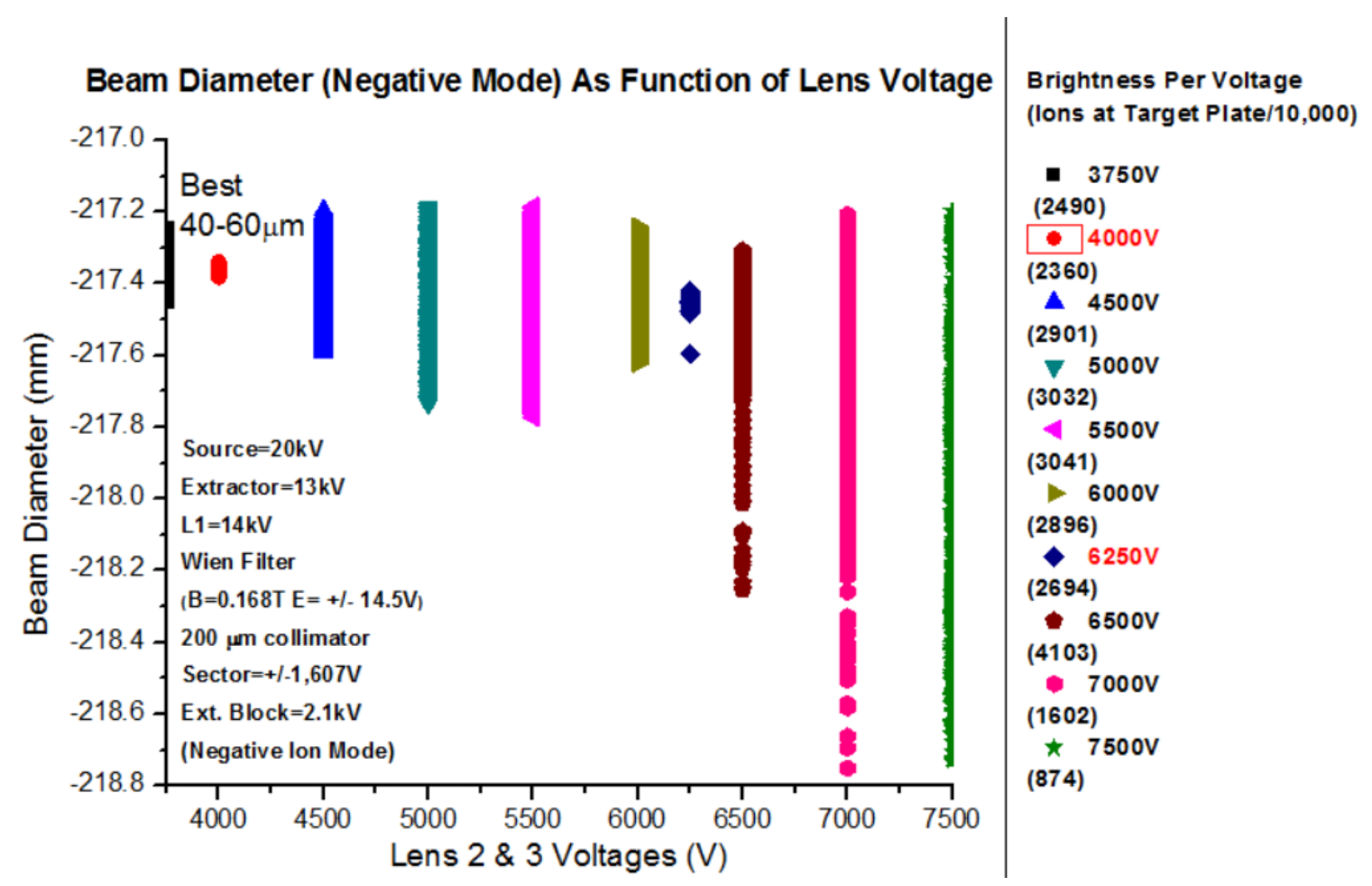

Figure 46. Results from the Negative Ion Mode Beam Profiling.

By sweeping the voltages as stated in Table 3 an optical voltage for positive and negative ion modes was found. Utilizing a voltage of $4 \mathrm{kV}$ in lens 2 and lens 3 produced the best compromise between focusing and cluster ion transmission, with an effective transmission of $20-30 \%$ and a beam diameter of $\sim 40-60 \mu \mathrm{m}$ in negative and positive modes. While there is another focusing point noted at $\sim 6250 \mathrm{~V}$ with a greater fluence, this produced a greater variance in beam diameter with associated losses. With these conditions, we can then expect a stable massive gold cluster DC beam with a routine spatial resolution of $50 \mu \mathrm{m}$. Something to note is that while these results do not indicate sub-micron spatial resolution in DC mode, this does not mean that the resolution is restricted to this number when utilizing the probe in a lower fluence, pulsed static SIMS regime. It may also be possible that the energy, mass and direction distributions may be too harsh (assuming worst case scenario), and better performance can be achieved 
experimentally. In addition, the Au-LMIS is not only limited to massive gold clusters, and perhaps sub-micron spatial resolution for dynamic SIMS could be achieved if utilizing other projectiles like $\mathrm{Au}_{5}{ }^{+}$or $\mathrm{Au}_{9}{ }^{+}$. These are not generated as part of a distribution like massive gold projectiles, and should present less of a challenge to focus while still providing enhanced yields when compared to $\mathrm{Bi}_{3}{ }^{+}$and $\mathrm{C}_{60}{ }^{+}$.
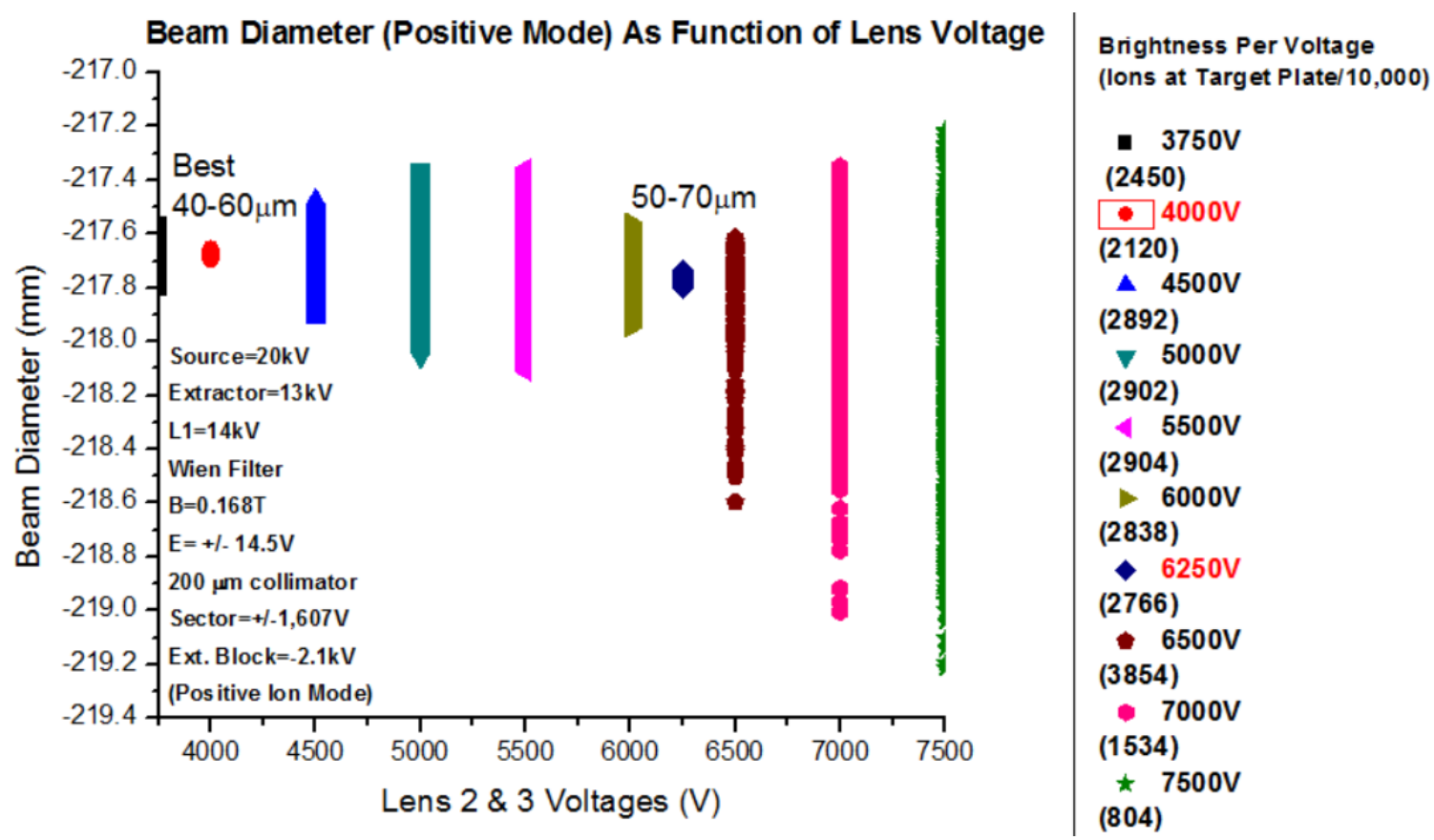

Figure 47. Results of the Positive Ion Mode Beam Profiling.

Evaluation of the SI lens began with simulations for the transmission of low $\mathrm{m} / \mathrm{z}$ ions (150-300 $\mathrm{m} / \mathrm{z})$. Emission parameters are those highlighted in Table 4 for low $\mathrm{m} / \mathrm{z}$ ions, with the exception of SI energies. These were accounted to have an initial energy spread based on a normal distribution with the limits being $0.02 \mathrm{eV}-0.08 \mathrm{eV}$. From these simulations, theoretical DC voltage values for optimal lens performance are with a target plate bias of $+50 \mathrm{~V},>90 \%$ transmission was achieved over 100 simulations with $-2.1 \mathrm{kV}$ on the extraction block/first electrode, $-500 \mathrm{~V}$ on the second electrode, $-450 \mathrm{~V}$ on the 
third electrode, $-250 \mathrm{~V}$ on the fourth electrode, $-150 \mathrm{~V}$ on the fifth electrode, $-30 \mathrm{~V}$ on the sixth electrode, and $0 \mathrm{~V}$ on the last electrode. A SIMION program was written to operate the quadrupole as a RF-only ion guide instead its usual mass-selective operation, at an amplitude $\left(\mathrm{V}_{\mathrm{pp}}\right)$ of $\pm 135 \mathrm{~V}$.

Injection energy, or the energy which the SIs are transferred into the quadrupole is of high importance as highly energetic ions will be lost. Specifications state the injection energy window for the SolariX is between $5-20 \mathrm{eV}$, with $\sim 10 \mathrm{eV}$ being an ideal injection energy. Mean injection energy for the ions in this simulation was roughly $\sim 15 \mathrm{eV}$, at the higher end of acceptable energy range for this quadrupole. The goal of this instrument however, is to look at large and intact molecular ions, thus transmission of heavier species is of interest. Further simulations were performed over a broader mass keeping the same voltage values, only slightly changing the RF on the quadrupole to act as a lowmass filter. Figure 48 shows an XY view of the final simulation consisting of three packets of 10,000 ions with varied mass distributions were flown simultaneously accounting for coulombic repulsion in flight (defined as $1 \times 10^{-12} \mathrm{~A}$, accounting for spacecharging during flight). Mass distributions are highlighted accounting for an expanse of $150-1100 \mathrm{~m} / \mathrm{z}$ singly charged ions. As stated in the methods, the target plate is positioned $5 \mathrm{~mm}$ away in these simulations, yet similar performance should be attainable at closer distances to further improve SI extraction efficiency. The ideal target plate potential to ensure max ion transmission can be simulated or experimentally determined as well. 


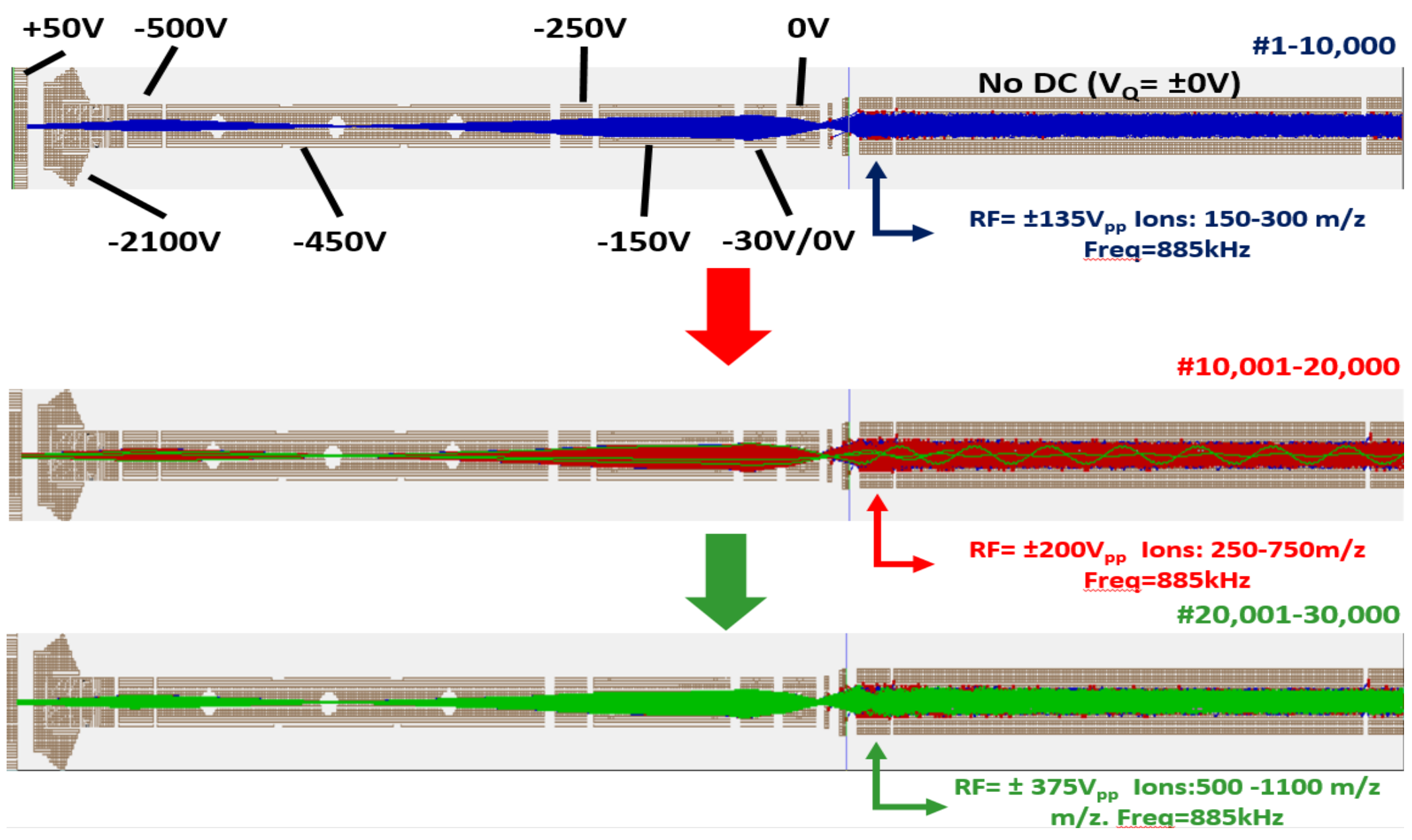

Figure 48. Simulation of optimized voltage performance for transmitting ions of varying mass into the first quadrupole of the SolariX, followed by the collisional cell (Q2). Ions of mass ranges $150-300 \mathrm{~m} / \mathrm{z}, 250-750 \mathrm{~m} / \mathrm{z}$, and $400-1100 \mathrm{~m} / \mathrm{z}$. Three packets of 10,000 ions were flown simultaneously accounting for coulombic repulsion. 


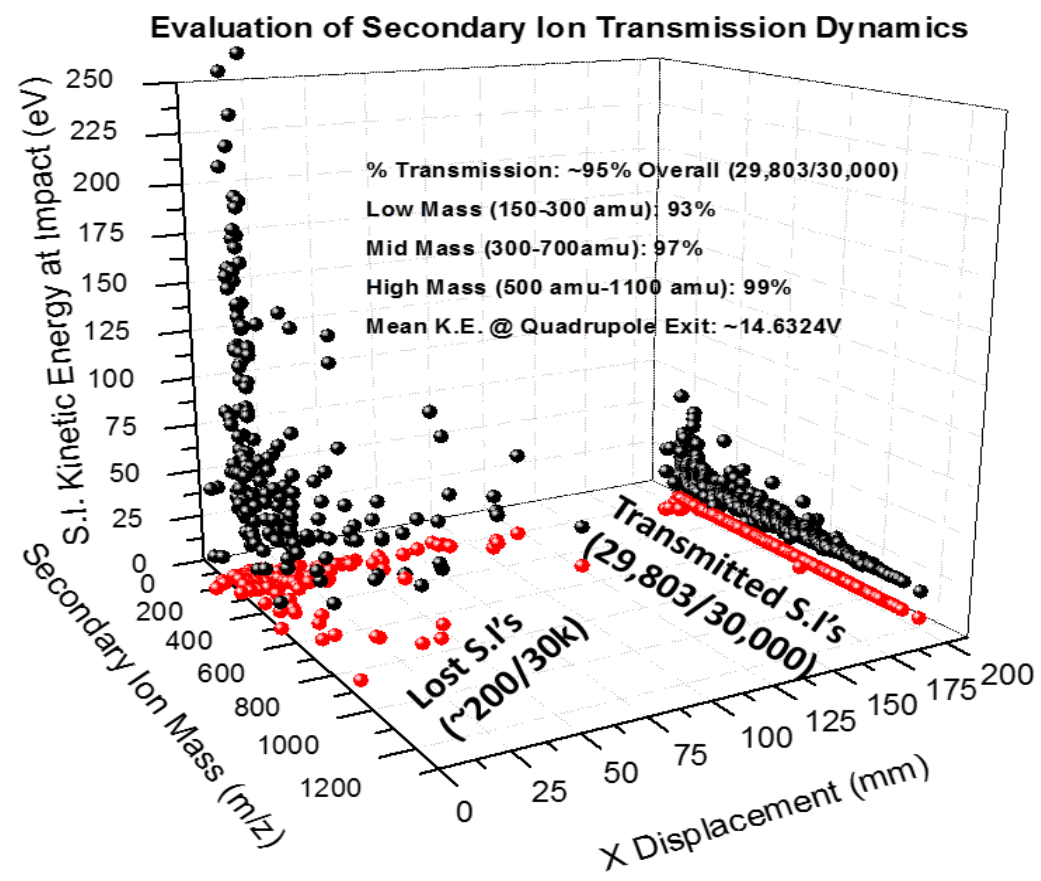

Figure 49. Evaluation of secondary ion transmission based on $m / z$ utilizing simulation derived values. Black data points accounting for energy, $m / z$, and $X$ displacement (transmission), while red data points account for displacement account for mass and displacement alone.

The SI behavior and subsequent fate (transmitted or lost) as a function of energy and $m / z$ is presented in Figure 49. Each data point represents an ion, with transmitted ions reaching the exit of the quadrupole. A clear trend shows that lighter ions were more difficult to transmit, generally acquiring more energy than the heavier species simulated. Transmissions averages for low, mid, and high mass ranges were 93\%, 97\%, and 98\%, respectively. Best possible results achieved were $98 \%$ transmission $(29,803 / 30,000)$ with a mean kinetic energy of $14.6 \mathrm{~V}$ prior to transfer into the collisional cell (Q2). Overall, simulation results show that the secondary extraction lens voltages are capable of transferring ions with no mass discrimination effects. Figure 50 shows a snapshot of the total simulation. 


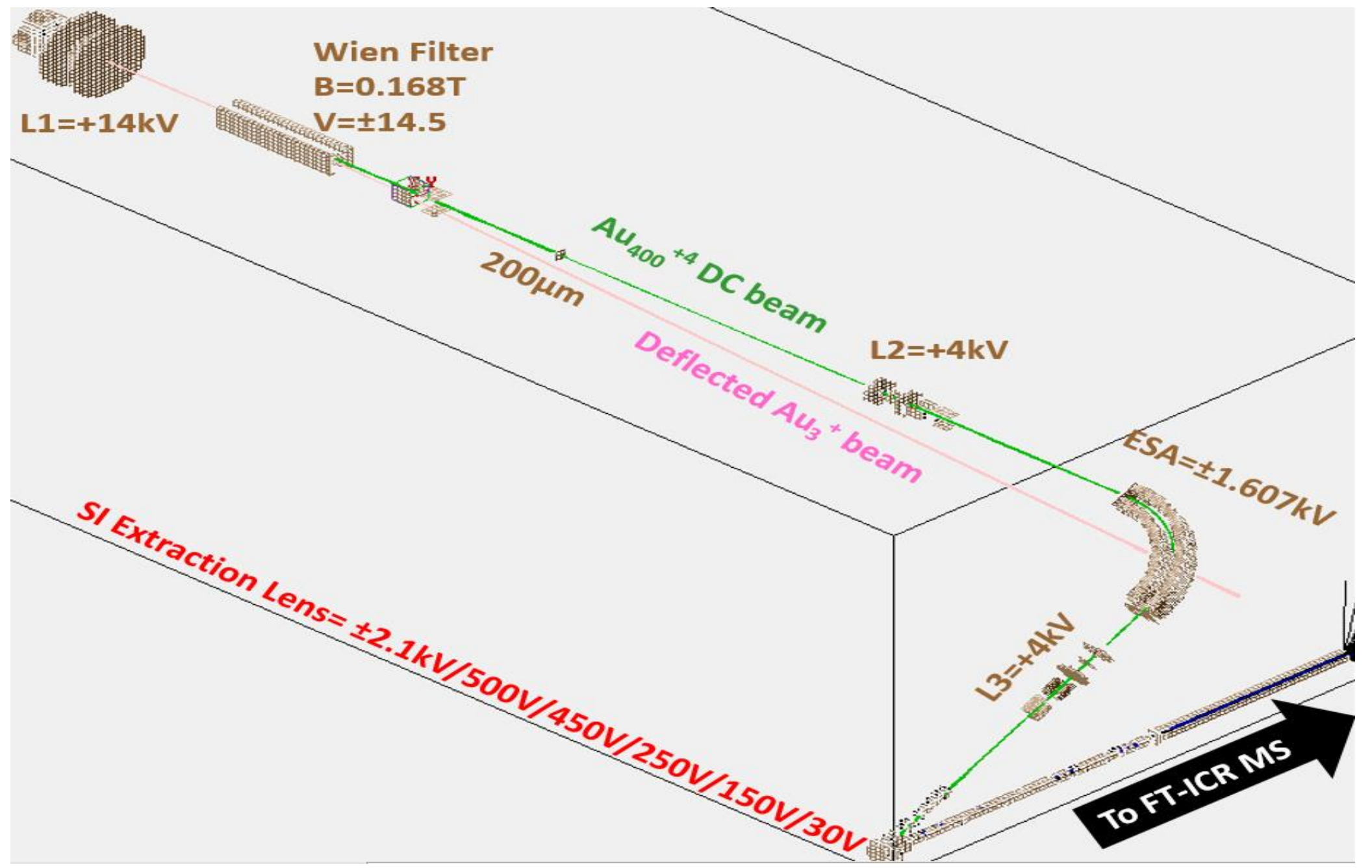

Figure 50. 3D view of the completed simulation with all optimized voltages highlighted. Simulation displays the trajectory of the focused primary ion beam and the transmission of the generated secondary ions into the FT-ICR MS Q1. 


\subsubsection{Construction of the Interfacing Optics and Final Instrument Assembly}

The manufacturing and fabrication of the components was custom-built at Florida International University - Physics Department and the Texas A \& M University Chemistry Department Machine Shops. The raw materials (e.g., steels, aluminum, insulating materials, wiring and wieldable connectors) were purchased from McMaster Carr (U.S.). Specialized high voltage, motion control, vacuum feedthroughs and flanges were purchased from Kurt Lesker (Jefferson Hills, PA) and MDC Vacuum (Hayward, CA).

Fabrication consisted of the electrostatic sector, housing, lens and extractor, modified housing for the FT-ICR MS and the Secondary Ion Lens Extraction Cartridge. The electrostatic sector and its housing (both bottom section and top lid) are fabricated from .316" ground steel (McMaster Carr). The entrance/exit slits were fabricated from sheet metal ( $\sim 0.5 \mathrm{~mm}$ thickness), and vacuum seal was achieved by adding a custom ordered Teflon rectangle O-ring for vacuum sealing (MDC Vacuum). Spacers to float the sector were made of PEEK. CF275 SHV feedthrough flanges to bias the sector segments were purchased from Kurt Lesker. The third einzel lens-extractor combination was fabricated from .316" ground steel (lens electrodes and deflector plates), a modified blank CF275 flange, and spacers made from PEEK. Both electrostatic sector and third einzel lens unit are powered by Matsuda $\pm \mathrm{AU}-20 \mathrm{kV}$ power supplies. The top of the housing that connects the incoming sector-bent beam into the extraction lens cartridge was fabricated of .316" stainless steel. Dimensions, materials and components in the firsthousing chamber will not be discussed due to a confidentiality agreement with Bruker regarding this information. The SI extraction lens block, cylinder electrodes, and 
supporting structures (horseshoe, support rods) were made from .316" stainless steel. Insulating spacers and lens support bars were made from PEEK and the central mounting ring was made from Teflon, all purchased from McMaster Carr. Holder for the MALDI window, LED, and camera feedthroughs were made from nylon purchased from McMasterr Carr. The LED was mounted on a small circuit board and soldered onto place. The SI extraction lens system is powered by a Glassman HV EL $-3 \mathrm{kV} / 15 \mathrm{~mA}$ power supply. Final assembly and testing was completed by 2015 .

\section{Vacuum Components}

In order to bring the source chamber to the desired pressure $\left(>10^{-7}\right.$ torr $)$ and avoid pressure differentials, an extra roughing pump and a turbomolecular pump were added at the source chamber. An Edwards EXT 255HP turbomolecular pump was added on the back part of the custom made housing, connected to an Edwards RV 12 HP Oil-sealed two stage rotary vane pump. These were connected to a pressure readout unit via a Pirani gauge. High-vacuum pressure readout is achieved utilizing the SolariX's onboard sensors and readout system. 


\section{PI/SI Electronics Rack AuNP-Primary Ion Gun Bruker Daltonics Solarix 7.0T FT-ICR MS}

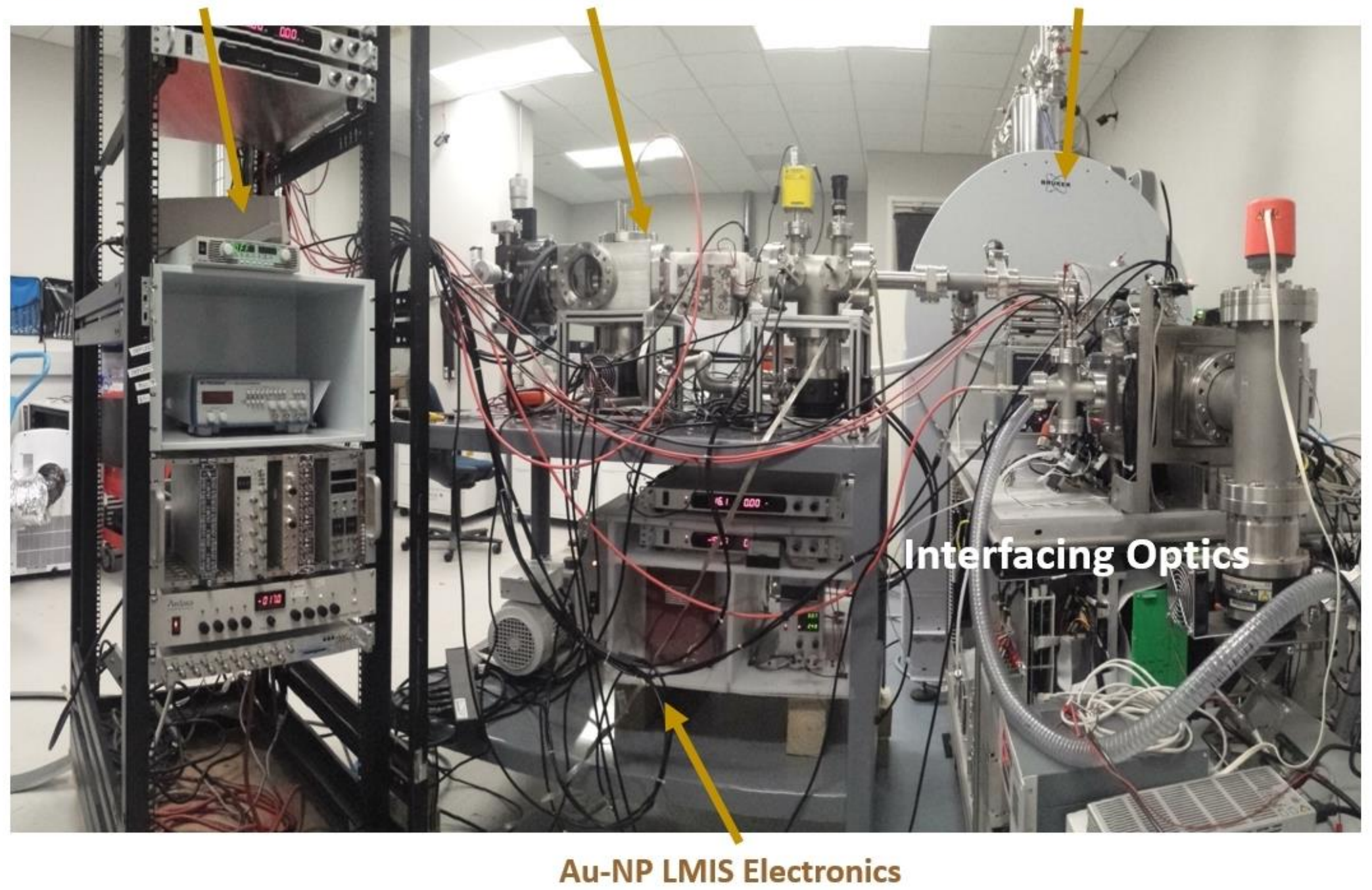

Figure 51. Side view of the completed AuNP-FT-ICR MS assembly with main components highlighted. 


\subsubsection{Experimental Evaluation of Secondary Ion Extraction Lens}

\subsubsection{Construction of Step-Down Resistor Chain for SI Lens}

Inputting the voltage values from the simulations displayed on Figure 48 into the expression sequence yields the potential differences between the electrodes required

$$
\begin{gathered}
-2100 \mathrm{~V} \stackrel{I * R 1=1600 \mathrm{~V}}{\longrightarrow}-500 \mathrm{~V} \stackrel{I * R 2=50 \mathrm{~V}}{\longrightarrow}-450 \mathrm{~V} \stackrel{I * R 3=200 \mathrm{~V}}{\longrightarrow}-250 \mathrm{~V} \stackrel{I * R 4=100 \mathrm{~V}}{\longrightarrow} \\
-150 \mathrm{~V} \stackrel{I * R 5=120 \mathrm{~V}}{\longrightarrow}-30 \mathrm{~V} \stackrel{I * R 6=30 \mathrm{~V}}{\longrightarrow} 0
\end{gathered}
$$

Knowing that the current is $1 \mathrm{~mA}$ across the entire circuit and the required voltage differences amongst the electrodes, the resistor values were calculated based on:

$$
\begin{gathered}
R_{\text {total }}=\sum \frac{1600 \mathrm{~V}}{0.001 A}+\frac{50 \mathrm{~V}}{0.001 A}+\frac{200 \mathrm{~V}}{0.001 A}+\frac{100 \mathrm{~V}}{0.001 A}+\frac{120 \mathrm{~V}}{0.001 A}+\frac{30 \mathrm{~V}}{0.001 A} \\
=1.6 \mathrm{M} \Omega+50 \mathrm{k} \Omega+200 \mathrm{k} \Omega+100 \mathrm{k} \Omega+120 \mathrm{k} \Omega+30 \mathrm{k} \Omega
\end{gathered}
$$

With the resistor values known, the experimental arrangement for resistors to achieve the desired voltages is defined as:

$-2100 \mathrm{~V} \stackrel{1.6 \mathrm{M} \Omega}{\longrightarrow}-500 \mathrm{~V} \stackrel{50 \mathrm{k} \Omega}{\longrightarrow}-450 \mathrm{~V} \stackrel{200 \mathrm{k} \Omega}{\longrightarrow}-250 \mathrm{~V} \stackrel{100 \mathrm{k} \Omega}{\longrightarrow}-150 \mathrm{~V} \stackrel{120 \mathrm{k} \Omega}{\longrightarrow}-30 \mathrm{~V} \stackrel{30 \mathrm{k} \Omega}{\longrightarrow} 0$

Resistors were purchased from DigiKey (Thief River Falls, MN) and Mouser (Mansfield, TX) to achieve voltages as close as possible to those from the simulations. These were connected in-series on a connector block outside of the vacuum envinronment. Having the ability of manipulating the resistors outside the vacuum environment allows for replacement in case of any performance issues without having to break vacuum conditions inside the experiment, minimizing down-time. Having assembled the lens and the electronics for their operation, the focus shifted towards the experimental validation of these values. 


\subsubsection{Development of a Vacuum Electrospray Source for Standalone Ion Optics Testing}

The initial prototype of this source was originally designed utilizing Solidworks

(Figure 52). While there are multiple applications, its purpose in this Thesis is to act as an ion source to experimentally validate the theoretically optimized voltages for the SI extraction lens. A blank CF2.75 flange purchased from Kurt Lesker, had a hole of $\sim 10$ $\mathrm{mm}$ bored in the center, and a .316 stainless steel nanocapillary threaded insert was welded in place. The net aperture diameter for capillary inserts is roughly $\sim 50 \mu \mathrm{m}$, suitable for fused silica/Kapton coated nanoelectrospray capillaries (nESI). Three 0.25 in deep 8-32 threaded holes were tapped onto the vacuum side of the flange, allowing for three 8-32 nylon or PEEK threaded posts to act as supports for mounting optics. For the earliest prototype iteration, 2.4 in nylon columns were cut. One spacer and one extractor made of .316 stainless steel sheet metal were placed on the column, with the spacer utilized for holding an insulated sleeve. The sleeveing was added to avoid motion of the emitter, results in unstable emission and capillary damage. Capillary fabrication procedures are listed in Appendix 2. The ionic liquid tributylmethylammonium chloride $\left(\mathrm{C}_{13} \mathrm{H}_{30} \mathrm{~N}-\mathrm{Cl}\right)$ was purchased from Sigma-Aldrich (St. Louis MO) and $1 \mathrm{~g}$ was mixed with $1 \mathrm{~mL}$ of $90 \% \mathrm{H} 20 / 10 \%$ Methanol to generate a concentrated $10 \mathrm{M}$ solution. A specialized alternating polarity high-voltage power supply was fabricated as well inhouse with components from DigiKey and Mauser (Figure 53). The power supply was made in order to prevent the occurrence of emitter fouling associated with ionic liquids beams $^{124}$. 
A.
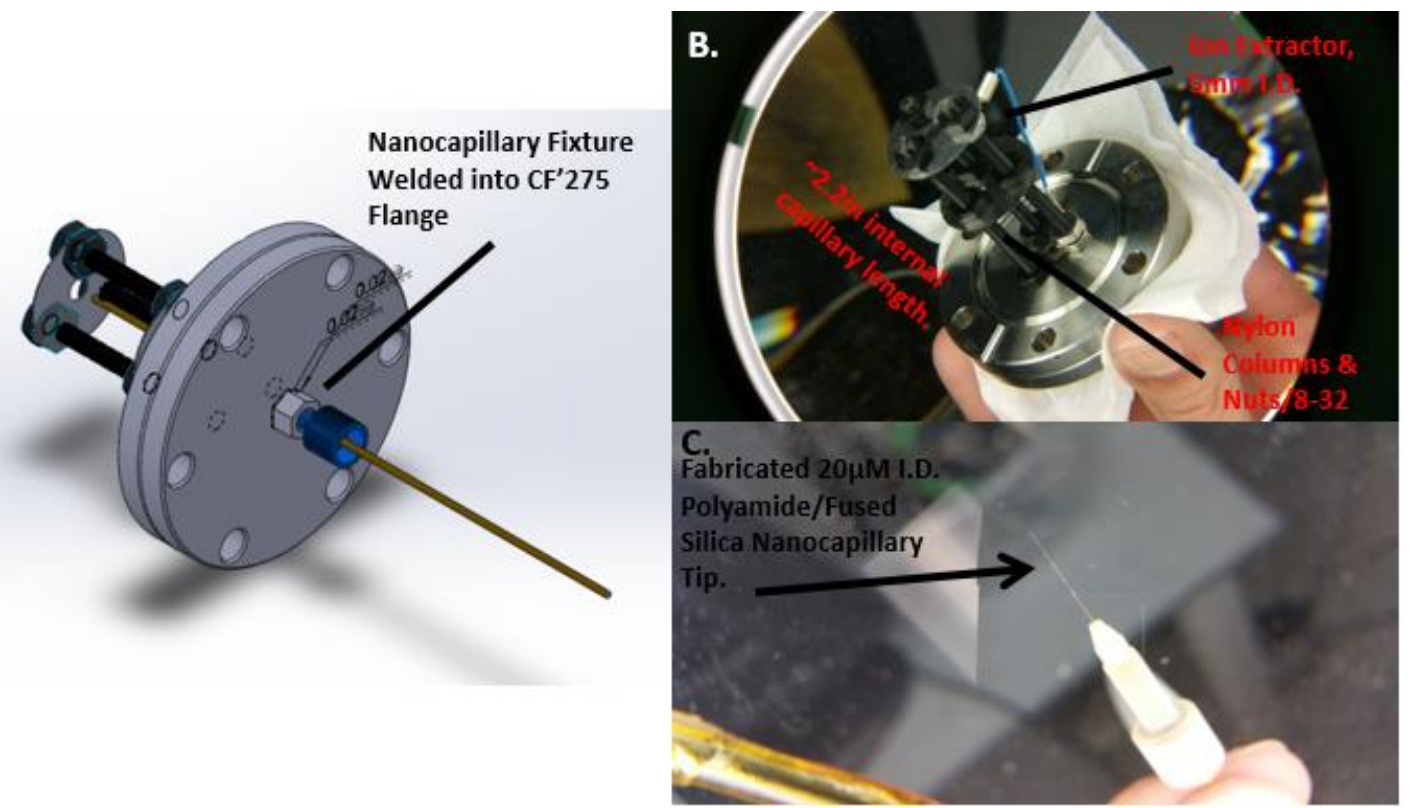

Figure 52. Design, fabrication and assembly of the vacuum electrospray source. A) Design for prototype source consists of a modified CF 2.75 in flange B). Photograph of assembled prototype flange. Total internal length is $\sim 2.2 \mathrm{in}$. C) Photograph of a freshly etched polyamide/fused silica nanoelectrospray capillary.

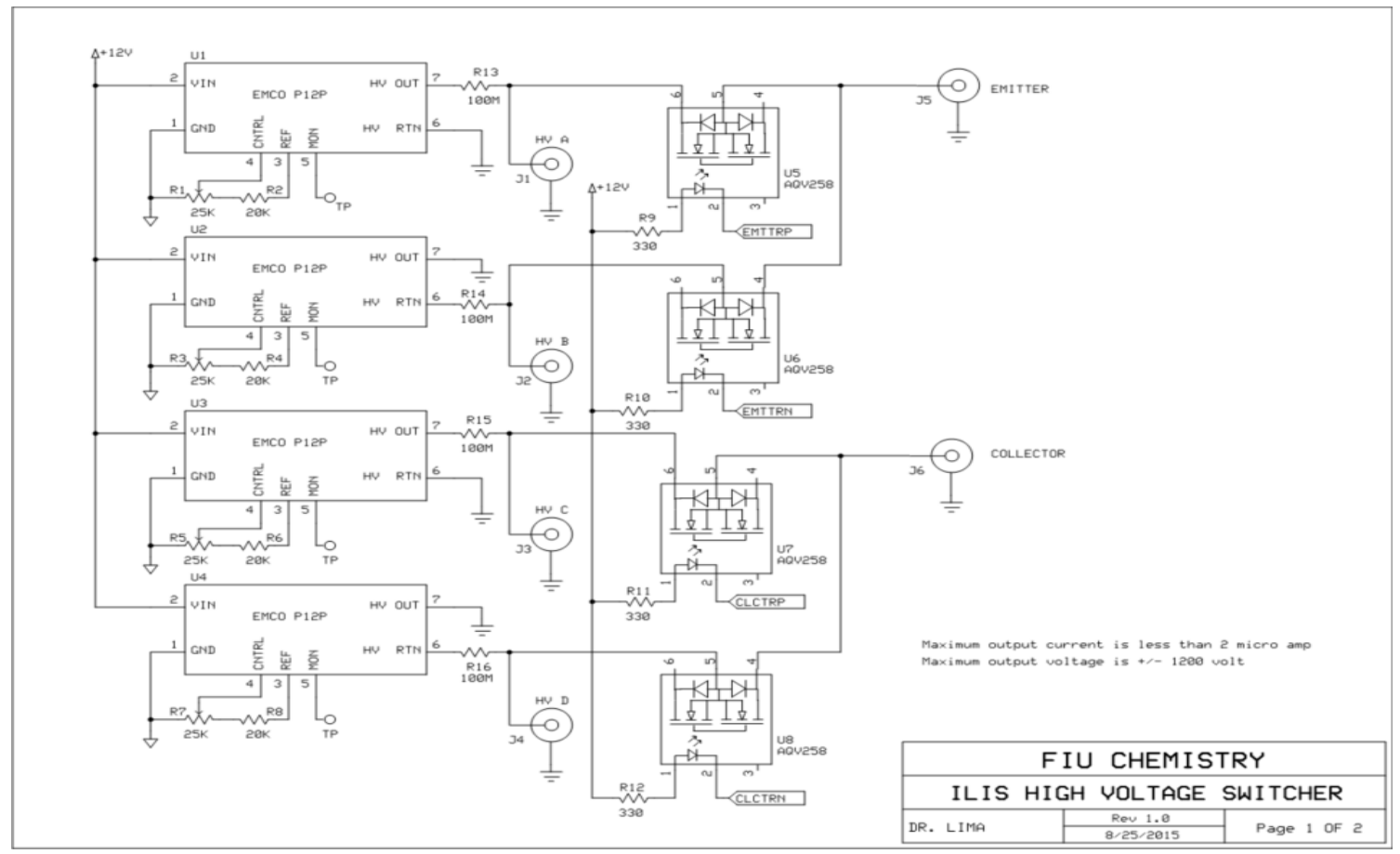

Figure 53. Electronics wiring diagram for alternating polarity HV power supply. 


\subsubsection{Assembly of the Test Bench and SI Lens Profiling}

As mentioned, design planning for the experimental bench was completed with the intention of housing the source and the lens. Figure 54 shows the assembled test bench with all the power supplies and components to generate the vacuum ionic liquid beam.

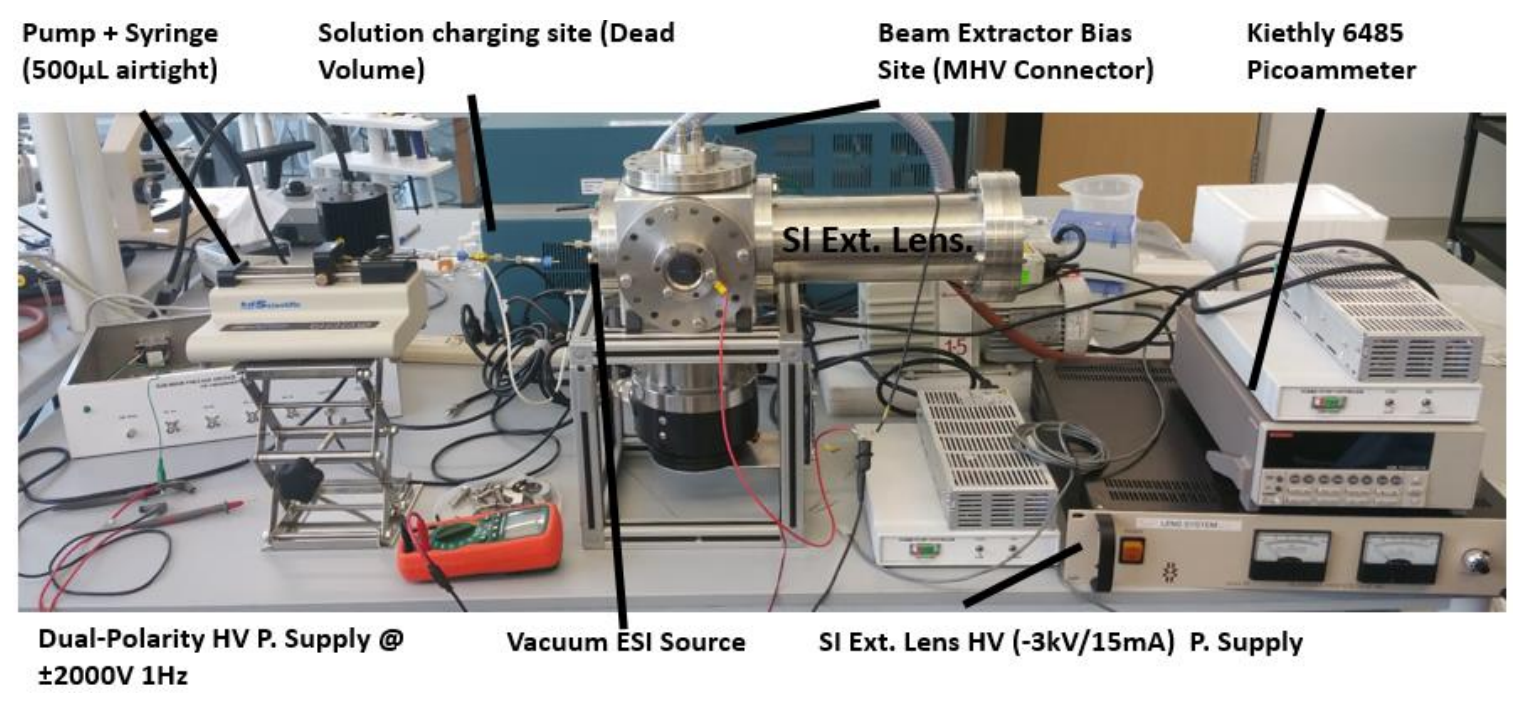

Figure 54. View of the assembled test bench with all relevant components labeled (with the exception of the 255HP Turbomolecular Pump). S.I. lens not pictured as these are located within the testing chamber.

In the test bench, the ion source was placed at a distance of $\sim 10 \mathrm{~mm}$ from the extractor to avoid discharging. Voltage values at the connectors are labeled in the Figure 54, with the corresponding resistance between each of the electrodes (labeled by color). Main difference in the experimental testing is that, as opposed to the simulations, the $6^{\text {th }}$ lens is grounded along with the $7^{\text {th }}$. This lens was rendered inactive due to discharging onto the housing during initial testing. 


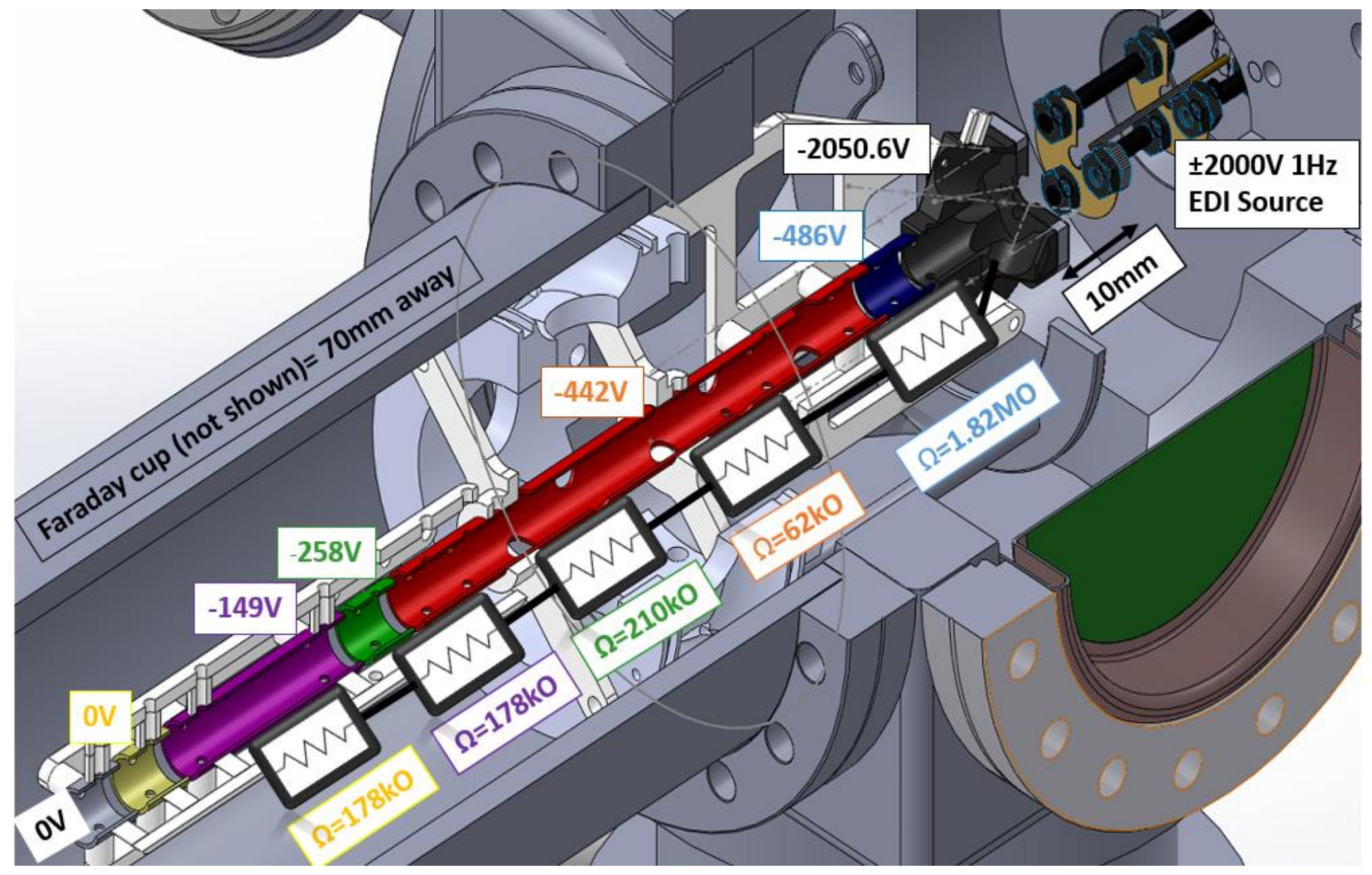

Figure 55. Solidworks cutaway view of test bench configuration for the evaluation of SI extraction lens transmission $(\mathrm{A} / \mathrm{sec})$. 
Results from the experimental test bench evaluation of the lens performance with the above voltages is displayed in Figure 56. Voltages were swept in increments of $500 \mathrm{~V}$, with current measurements taken every 5 seconds for a duration of 10 minutes, producing 120 current measurements. As formulated by the simulations, the designed resistor chain provided a significant amount of ion transmission, even at a small target $(6 \mathrm{~mm})$ at a distance of $70 \mathrm{~mm}$, roughly the distance to halfway through Q1. These results validate the constructed resistor chain for the lens to produce voltages which guarantee SI transmission into the first quadrupole of the SolariX and beyond.

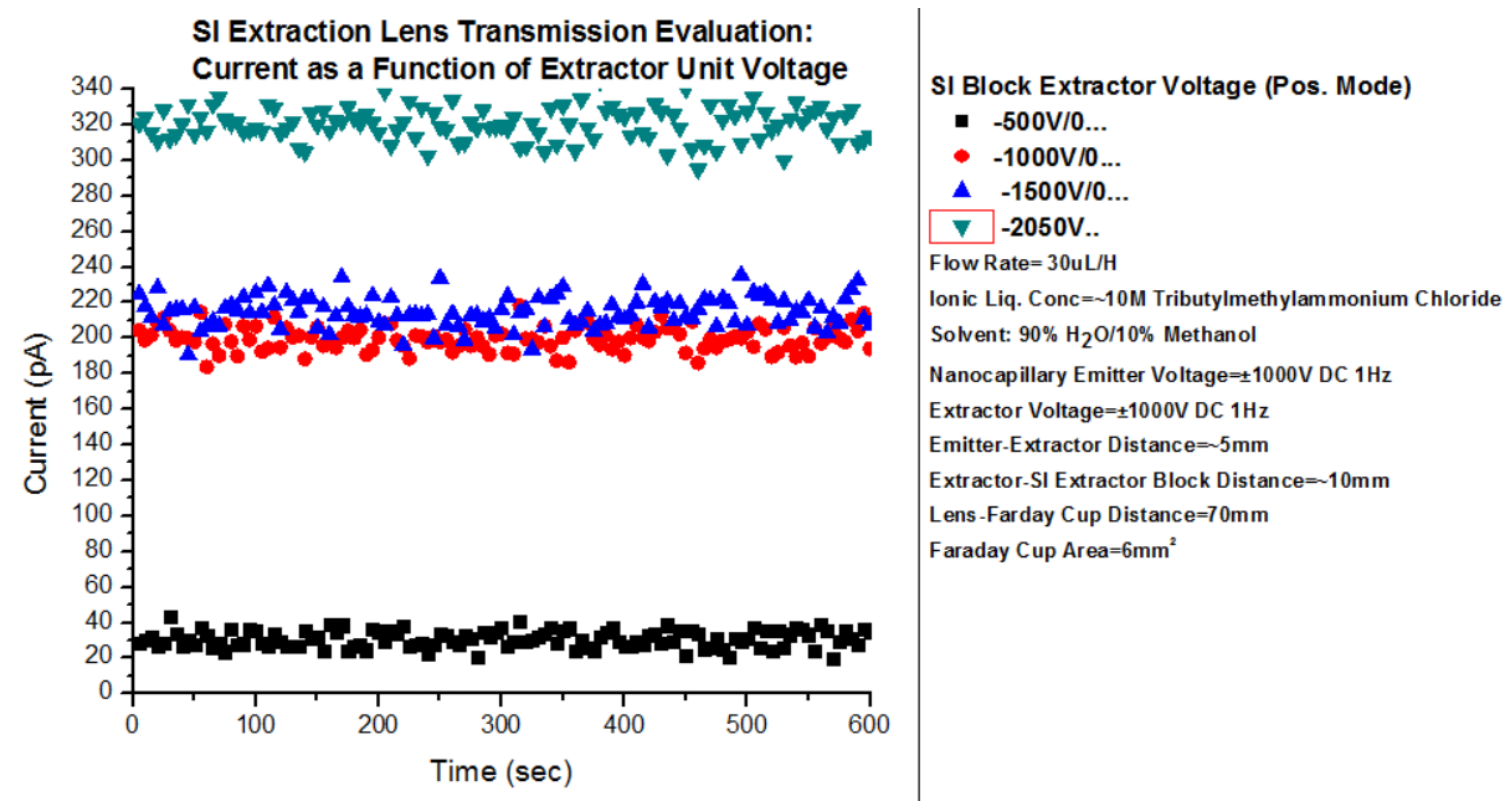

Figure 56. Experimental Ion Transmission Evaluation of the Secondary Ion Extraction Lens as a function of extractor block voltage. Note that max transmission is achieved with the theorized simulation value. 


\subsection{Conclusion}

The present work shows the advances of theoretical modeling in combination with experimental evaluation for the coupling of a AuNP-LMIS $\left(\mathrm{Au}_{400}{ }^{+4}\right)$ equipped PI column, to a Bruker Daltonics SolariX FT-ICR MS. Interfacing optics to annex the PI column and SI extraction optics were designed, theoretically evaluated, and experimentally tested. A new design for an electrostatic interface between the PI column and the FT-ICR-MS has been proposed and evaluated. Preliminary simulations of the optimized PI gun and interfacing optics showed that a DC beam consisting of a wide distribution of massive gold clusters can be mass-filtered and focused to a beam diameter of $\sim 50 \mu \mathrm{m}$. Secondary ion transmission can be achieved with over $~ 95 \%$ efficacy with a narrow energy profile for secondary ions. Transmission of medium to large molecular ions was routinely achieved utilizing fixed settings with a low-mass cut off inherent to the FT-ICR MS. A vacuum electrospray system was designed in order to examine the performance of ion optics in a standalone environment, and shows promise as a source for cluster projectiles as well. An experimental test bench was built to assess the performance of the SI extraction lens, with positives results for ion transmission obtained with simulationderived values. Based on the combination of theoretical and experimental data, we anticipate that this platform will be capable of routinely characterizing surfaces using a $\mathrm{Au}_{400}{ }^{+4} \mathrm{DC}$ beam with a mass resolution of over $400 \mathrm{k}$ at $400 \mathrm{~m} / \mathrm{z}$. 


\section{References}

1. Dowsett, M. G.; Barlow, R. D.; Allen, P. N., Secondary ion mass spectrometry analysis of ultrathin impurity layers in semiconductors and their use in quantification, instrumental assessment, and fundamental measurements. Journal of Vacuum Science \& Technology B 1994, 12 (1), 186-198.

2. Vickerman, J. C.; Gilmore, I. S., Surface analysis: the principal techniques. Wiley Online Library: 2009; Vol. 2.

3. Heeren, R. M. A., Getting the picture: The coming of age of imaging MS. International Journal of Mass Spectrometry 2015, 377, 672-680.

4. McDonnell, L. A.; Heeren, R. M. A., Imaging mass spectrometry. Mass Spectrometry Reviews 2007, 26 (4), 606-643.

5. Wu, C.; Dill, A. L.; Eberlin, L. S.; Cooks, R. G.; Ifa, D. R., Mass spectrometry imaging under ambient conditions. Mass Spectrometry Reviews 2013, 32 (3), 218-243.

6. Seeley, E. H.; Caprioli, R. M., Imaging mass spectrometry: Towards clinical diagnostics. PROTEOMICS - Clinical Applications 2008, 2 (10-11), 1435-1443.

7. Wiseman, J. M.; Ifa, D. R.; Zhu, Y.; Kissinger, C. B.; Manicke, N. E.; Kissinger, P. T.; Cooks, R. G., Desorption electrospray ionization mass spectrometry: Imaging drugs and metabolites in tissues. Proceedings of the National Academy of Sciences 2008, 105 (47), 18120-18125.

8. Chabala, J. M.; Levi-Setti, R.; Wang, Y. L., Practical resolution limits of imaging microanalysis with a scanning ion microprobe. Applied Surface Science 1988, 32 (1), 1032.

9. Gode, D.; Volmer, D. A., Lipid imaging by mass spectrometry - a review. Analyst 2013, 138 (5), 1289-1315.

10. Van der Heide, P., Secondary Ion Mass Spectrometry: An Introduction to Principles and Practices. John Wiley \& Sons: 2014.

11. Mahoney, C. M.; Gillen, G., An Introduction to Cluster Secondary Ion Mass Spectrometry (Cluster SIMS). In Cluster Secondary Ion Mass Spectrometry, John Wiley \& Sons, Inc.: 2013; pp 1-11.

12. Zenobi, R.; Knochenmuss, R., Ion formation in MALDI mass spectrometry. Mass Spectrometry Reviews 1998, 17 (5), 337-366. 
13. Nemes, P.; Vertes, A., Laser Ablation Electrospray Ionization for Atmospheric Pressure, in Vivo, and Imaging Mass Spectrometry. Analytical Chemistry 2007, 79 (21), 8098-8106.

14. Swales, J. G.; Tucker, J. W.; Spreadborough, M. J.; Iverson, S. L.; Clench, M. R.; Webborn, P. J. H.; Goodwin, R. J. A., Mapping Drug Distribution in Brain Tissue Using Liquid Extraction Surface Analysis Mass Spectrometry Imaging. Analytical Chemistry 2015, 87 (19), 10146-10152.

15. Winograd, N., Imaging Mass Spectrometry on the Nanoscale with Cluster Ion Beams. Analytical Chemistry 2015, 87 (1), 328-333.

16. Benninghoven, A., Static SIMS applications-From silicon single crystal oxidation to DNA sequencing. Journal of Vacuum Science \& Technology A 1985, 3 (3), 451-460.

17. Benninghoven, A., Chemical Analysis of Inorganic and Organic Surfaces and Thin Films by Static Time-of-Flight Secondary Ion Mass Spectrometry (TOF-SIMS). Angewandte Chemie International Edition in English 1994, 33 (10), 1023-1043.

18. Gillen, G.; Simons, D. S.; Williams, P., Molecular ion imaging and dynamic secondary-ion mass spectrometry of organic compounds. Analytical Chemistry 1990, 62 (19), 2122-2130.

19. Winograd, N., The Magic of Cluster SIMS. Analytical Chemistry 2005, 77 (7), 142 A-149 A.

20. Mahoney, C. M., Cluster secondary ion mass spectrometry of polymers and related materials. Mass Spectrometry Reviews 2010, 29 (2), 247-293.

21. Gilmore, I. S., SIMS of organics-Advances in 2D and 3D imaging and future outlook. Journal of Vacuum Science \& Technology A 2013, 31 (5), 050819.

22. Havelund, R.; Licciardello, A.; Bailey, J.; Tuccitto, N.; Sapuppo, D.; Gilmore, I. S.; Sharp, J. S.; Lee, J. L. S.; Mouhib, T.; Delcorte, A., Improving Secondary Ion Mass Spectrometry C60n+ Sputter Depth Profiling of Challenging Polymers with Nitric Oxide Gas Dosing. Analytical Chemistry 2013, 85 (10), 5064-5070.

23. Shard, A. G.; Havelund, R.; Seah, M. P.; Spencer, S. J.; Gilmore, I. S.; Winograd, N.; Mao, D.; Miyayama, T.; Niehuis, E.; Rading, D.; Moellers, R., Argon Cluster Ion Beams for Organic Depth Profiling: Results from a VAMAS Interlaboratory Study. Analytical Chemistry 2012, 84 (18), 7865-7873.

24. Pachuta, S. J.; Cooks, R. G., Mechanisms in molecular SIMS. Chemical Reviews 1987, 87 (3), 647-669. 
25. Sigmund, P.; Claussen, C., Sputtering from elastic-collision spikes in heavy-ionbombarded metals. Journal of Applied Physics 1981, 52 (2), 990-993.

26. Sigmund, P., Theory of Sputtering. I. Sputtering Yield of Amorphous and Polycrystalline Targets. Physical Review 1969, 184 (2), 383-416.

27. Rabalais, J. W.; Chen, J. N., Inelastic processes in ion/surface collisions: Direct recoil ion fractions as a function of kinetic energy. The Journal of Chemical Physics 1986, 85 (6), 3615-3622.

28. Magee, C. W., Sputtering of organic molecules. International Journal of Mass Spectrometry and Ion Physics 1983, 49 (2), 211-221.

29. Williams, P., The sputtering process and sputtered ion emission. Surface Science 1979, 90 (2), 588-634.

30. Andersen, C. A.; Hinthorne, J. R., Thermodynamic approach to the quantitative interpretation of sputtered ion mass spectra. Analytical Chemistry 1973, 45 (8), 14211438.

31. Winograd, N.; Garrison, B. J., Surface Structure and Reaction Studies by Ion-Solid Collisions. In Ion Spectroscopies for Surface Analysis, Czanderna, A. W.; Hercules, D. M., Eds. Springer US: Boston, MA, 1991; pp 45-141.

32. Fallavier, M., Secondary electron emission of solids by impact of molecular ions and clusters. Nuclear Instruments and Methods in Physics Research Section B: Beam Interactions with Materials and Atoms 1996, 112 (1-4), 72-78.

33. Bouneau, S.; Della Negra, S.; Jacquet, D.; Le Beyec, Y.; Pautrat, M.; Shapiro, M. H.; Tombrello, T. A., Measurement of energy and angular distributions of secondary ions in the sputtering of gold by swift $\$\{\backslash \text { mathrm }\{\mathrm{Au}\}\}_{-}\{\mathrm{n}\} \$$ clusters: Study of emission mechanisms. Physical Review B 2005, 71 (17), 174110.

34. Slodzian, G., Some problems encountered in secondary ion emission applied to elementary analysis. Surface Science 1975, 48 (1), 161-186.

35. Gnaser, H., Evidence of bond-breaking processes in sputtered ion emission. International Journal of Mass Spectrometry and Ion Processes 1984, 61 (1), 81-86.

36. Yu, M. L.; Lang, N. D., Direct Evidence of Electron Tunneling in the Ionization of Sputtered Atoms. Physical Review Letters 1983, 50 (2), 127-130.

37. Yu, M. L.; Lang, N. D., Mechanisms of atomic ion emission during sputtering. Nuclear Instruments and Methods in Physics Research Section B: Beam Interactions with Materials and Atoms 1986, 14 (4-6), 403-413. 
38. Honda, F.; Lancaster, G. M.; Fukuda, Y.; Rabalais, J. W., SIMS study of the mechanism of cluster formation during ion bombardment of alkali halides. The Journal of Chemical Physics 1978, 69 (11), 4931-4937.

39. Murray, P. T.; Rabalais, J. W., Ejection dynamics and electronic processes governing secondary particle emission in SIMS. Journal of the American Chemical Society 1981, 103 (5), 1007-1013.

40. Benninghoven, A., Some aspects of secondary ion mass spectrometry of organic compounds. International Journal of Mass Spectrometry and Ion Physics 1983, 53, 8599.

41. Michl, J., Secondary ion mass spectrometry of low-temperature solids. International Journal of Mass Spectrometry and Ion Physics 1983, 53, 255-272.

42. David, D. E.; Magnera, T. F.; Tian, R.; Stulik, D.; Michl, J., Cluster ions from keVenergy ion and atom bombardment of frozen gases. Nuclear Instruments and Methods in Physics Research Section B: Beam Interactions with Materials and Atoms 1986, 14 (46), 378-391.

43. Coles, J. N., A study of the feasibility of a surface plasma influencing secondary ion and photon emission under medium-energy ion bombardment *. Surface Science 1979, 79 (2), 549-574.

44. Unger, S. E.; Day, R. J.; Cooks, R. G., Possitive and negative secondary ion mass spectra and mass-analyzed ion kinetic energy spectra of some amides, amines and related compounds: Mechanisms in molecular sims. International Journal of Mass Spectrometry and Ion Physics 1981, 39 (2), 231-255.

45. Cooks, R. G.; Busch, K. L., Matrix effects, internal energies and MS/MS spectra of molecular ions sputtered from surfaces. International Journal of Mass Spectrometry and Ion Physics 1983, 53, 111-124.

46. Garrison, B. J.; Delcorte, A.; Krantzman, K. D., Molecule Liftoff from Surfaces. Accounts of Chemical Research 2000, 33 (2), 69-77.

47. Delcorte, A.; Wojciechowski, I.; Gonze, X.; Garrison, B. J.; Bertrand, P., Single and double cationization of organic molecules in SIMS. International Journal of Mass Spectrometry 2002, 214 (2), 213-232.

48. Harrison, D. E., Application of molecular dynamics simulations to the study of ionbombarded metal surfaces. Critical Reviews in Solid State and Materials Sciences 1988, 14 (sup1), s1-s78. 
49. Delcorte, A.; Garrison, B. J.; Hamraoui, K., Dynamics of Molecular Impacts on Soft Materials: From Fullerenes to Organic Nanodrops. Analytical Chemistry 2009, 81 (16), 6676-6686.

50. Delcorte, A.; Garrison, B. J., Desorption of large molecules with light-element clusters: Effects of cluster size and substrate nature. Nuclear Instruments and Methods in Physics Research Section B: Beam Interactions with Materials and Atoms 2011, 269 (14), 1572-1577.

51. Jakas, M. M.; Harrison, D. E., Many-body effects in atomic-collision cascades. Physical Review Letters 1985, 55 (17), 1782-1785.

52. Ziegler, J. F., The stopping and range of ions in solids / J.F. Ziegler, J.P. Biersack, U. Littmark. Pergamon: New York, 1985.

53. Garrison, B. J.; Postawa, Z., Computational view of surface based organic mass spectrometry. Mass Spectrometry Reviews 2008, 27 (4), 289-315.

54. Mahoney, J. F.; Yahiku, A. Y.; Daley, H. L.; Moore, R. D.; Perel, J., Electrohydrodynamic Ion Source. Journal of Applied Physics 1969, 40 (13), 5101-5106.

55. Krohn, V. E.; Ringo, G. R., Ion source of high brightness using liquid metal. Applied Physics Letters 1975, 27 (9), 479-481.

56. Sudraud, P.; Van De Walle, J.; Colliex, C.; Castaing, R., Contribution of field effects to the achievement of higher brightness ion sources. Surface Science 1978, 70 (1), 392402.

57. Clampitt, R.; Jefferies, D. K., Miniature ion sources for analytical instruments. Nuclear Instruments and Methods 1978, 149 (1), 739-742.

58. Swanson, L. W.; Schwind, G. A.; Bell, A. E.; Brady, J. E., Emission characteristics of gallium and bismuth liquid metal field ion sources. Journal of Vacuum Science \& Technology 1979, 16 (6), 1864-1867.

59. Lejeune, C., Theoretical and experimental study of the duoplasmatron ion source. Nuclear Instruments and Methods 1974, 116 (3), 417-428.

60. Schwieters, J.; Cramer, H. G.; Heller, T.; Jürgens, U.; Niehuis, E.; Zehnpfenning, J.; Benninghoven, A., High mass resolution surface imaging with a time-of-flight secondary ion mass spectroscopy scanning microprobe. Journal of Vacuum Science \& Technology A 1991, 9 (6), 2864-2871. 
61. Guerquin-Kern, J.-L.; Wu, T.-D.; Quintana, C.; Croisy, A., Progress in analytical imaging of the cell by dynamic secondary ion mass spectrometry (SIMS microscopy). Biochimica et Biophysica Acta (BBA) - General Subjects 2005, 1724 (3), 228-238.

62. Musat, N.; Halm, H.; Winterholler, B.; Hoppe, P.; Peduzzi, S.; Hillion, F.; Horreard, F.; Amann, R.; Jørgensen, B. B.; Kuypers, M. M. M., A single-cell view on the ecophysiology of anaerobic phototrophic bacteria. Proceedings of the National Academy of Sciences 2008, 105 (46), 17861-17866.

63. Chandra, S.; Ahmad, T.; Barth, R. F.; Kabalka, G. W., Quantitative evaluation of boron neutron capture therapy (BNCT) drugs for boron delivery and retention at subcellular-scale resolution in human glioblastoma cells with imaging secondary ion mass spectrometry (SIMS). Journal of Microscopy 2014, 254 (3), 146-156.

64. Torgerson, D. F.; Skowronski, R. P.; Macfarlane, R. D., New approach to the mass spectroscopy of non-volatile compounds. Biochemical and Biophysical Research Communications 1974, 60 (2), 616-621.

65. McNeal, C. J.; Macfarlane, R. D., Observation of a fully protected oligonucleotide dimer at m/z 12637 by californium-252 plasma desorption mass spectrometry. Journal of the American Chemical Society 1981, 103 (6), 1609-1610.

66. Andersen, H. H.; Bay, H. L., Nonlinear effects in heavy-ion sputtering. Journal of Applied Physics 1974, 45 (2), 953-954.

67. Andersen, H. H.; Bay, H. L., Heavy-ion sputtering yields of gold: Further evidence of nonlinear effects. Journal of Applied Physics 1975, 46 (6), 2416-2422.

68. Appelhans, A. D.; Delmore, J. E., Comparison of polyatomic and atomic primary beams for secondary ion mass spectrometry of organics. Analytical Chemistry 1989, 61 (10), 1087-1093.

69. Blain, M. G.; Della-Negra, S.; Joret, H.; Le Beyec, Y.; Schweikert, E. A., Secondary-ion yields from surfaces bombarded with keV molecular and cluster ions. Physical Review Letters 1989, 63 (15), 1625-1628.

70. Attal, P.; Della-Negra, S.; Gardès, D.; Larson, J. D.; Le Beyec, Y.; Vienet-Legué, R.; Waast, B., ORION project: acceleration of ion clusters and highly charged biomolecules from $10 \mathrm{MeV}$ to $1 \mathrm{GeV}$. Nuclear Instruments and Methods in Physics Research Section A: Accelerators, Spectrometers, Detectors and Associated Equipment 1993, 328 (1), 293299.

71. Waast, B.; Della-Negra, S.; Lafoux, A., Acceleration of heavy clusters to MeV energies at the Orsay MP tandem. Nuclear Instruments and Methods in Physics Research 
Section A: Accelerators, Spectrometers, Detectors and Associated Equipment 1996, 382 (1-2), 348-356.

72. Baudin, K.; Brunelle, A.; Della-Negra, S.; Jacquet, D.; Håkansson, P.; Le Beyec, Y.; Pautrat, M.; Pinho, R. R.; Schoppmann, C., Sputtering of large size clusters from solids bombarded by high energy cluster ions and fullerenes. Nuclear Instruments and Methods in Physics Research Section B: Beam Interactions with Materials and Atoms 1996, 112 (1-4), 59-63.

73. Benguerba, M.; Brunelle, A.; Della-Negra, S.; Depauw, J.; Joret, H.; Le Beyec, Y.; Blain, M. G.; Schweikert, E. A.; Assayag, G. B.; Sudraud, P., Impact of slow gold clusters on various solids: nonlinear effects in secondary ion emission. Nuclear Instruments and Methods in Physics Research Section B: Beam Interactions with Materials and Atoms 1991, 62 (1), 8-22.

74. Schoppmann, C.; Wohlfart, P.; Brandl, D.; Sauer, M.; Tomaschko, C.; Voit, H.; Boussofiane, K.; Brunelle, A.; Chaurand, P.; Depauw, J.; Della-Negra, S.; Håkansson, P.; LeBeyec, Y., Acceleration of Aun clusters to MeV energies. Nuclear Instruments and Methods in Physics Research Section B: Beam Interactions with Materials and Atoms 1993, 82 (1), 156-160.

75. Della-Negra, S.; Brunelle, A.; Le Beyec, Y.; Curaudeau, J. M.; Mouffron, J. P.; Waast, B.; Håkansson, P.; Sundqvist, B. U. R.; Parilis, E., Acceleration of Cn+60 molecules to high energy. Nuclear Instruments and Methods in Physics Research Section B: Beam Interactions with Materials and Atoms 1993, 74 (3), 453-456.

76. Boussofiane-Baudin, K.; Bolbach, G.; Brunelle, A.; Della-Negra, S.; Håkansson, P.; Le Beyec, Y., Secondary ion emission under cluster impact at low energies $(5-60 \mathrm{keV})$; influence of the number of atoms in the projectile. Nuclear Instruments and Methods in Physics Research Section B: Beam Interactions with Materials and Atoms 1994, 88 (1), 160-163.

77. Schweikert, E. A.; van Stipdonk, M. J.; Harris, R. D., A Comparison of Desorption Yields from C+60 to Atomic and Polyatomic Projectiles at keV Energies. Rapid Communications in Mass Spectrometry 1996, 10 (15), 1987-1991.

78. Gillen, G.; Roberson, S., Preliminary evaluation of an SF5+ polyatomic primary ion beam for analysis of organic thin films by secondary ion mass spectrometry. Rapid Communications in Mass Spectrometry 1998, 12 (19), 1303-1312.

79. Davies, N.; Weibel, D. E.; Blenkinsopp, P.; Lockyer, N.; Hill, R.; Vickerman, J. C., Development and experimental application of a gold liquid metal ion source. Applied Surface Science 2003, 203-204, 223-227. 
80. Touboul, D.; Halgand, F.; Brunelle, A.; Kersting, R.; Tallarek, E.; Hagenhoff, B.; Laprévote, O., Tissue Molecular Ion Imaging by Gold Cluster Ion Bombardment. Analytical Chemistry 2004, 76 (6), 1550-1559.

81. Nagy, G.; Gelb, L. D.; Walker, A. V., An Investigation of Enhanced Secondary Ion Emission Under Aun+ $(\mathrm{n}=1-7)$ Bombardment. Journal of the American Society for Mass Spectrometry 2005, 16 (5), 733-742.

82. Kollmer, F., Cluster primary ion bombardment of organic materials. Applied Surface Science 2004, 231-232, 153-158.

83. Touboul, D.; Kollmer, F.; Niehuis, E.; Brunelle, A.; Laprévote, O., Improvement of Biological Time-of-Flight-Secondary Ion Mass Spectrometry Imaging with a Bismuth Cluster Ion Source. Journal of the American Society for Mass Spectrometry 2005, 16 (10), 1608-1618.

84. Nagy, G.; Lu, P.; Walker, A. V., An investigation of secondary ion yield enhancement using Bin2+ (n=1,3,5) primary ions. J Am Soc Mass Spectrom 2008, 19 (1), $33-45$.

85. (a) Weibel, D.; Wong, S.; Lockyer, N.; Blenkinsopp, P.; Hill, R.; Vickerman, J. C., A C60 Primary Ion Beam System for Time of Flight Secondary Ion Mass Spectrometry: Its Development and Secondary Ion Yield Characteristics. Analytical Chemistry 2003, 75 (7), 1754-1764; (b) Hill, R.; Blenkinsopp, P. W. M., The development of C60 and gold cluster ion guns for static SIMS analysis. Applied Surface Science 2004, 231-232, 936939.

86. Cheng, J.; Wucher, A.; Winograd, N., Molecular Depth Profiling with Cluster Ion Beams. The Journal of Physical Chemistry B 2006, 110 (16), 8329-8336.

87. Passarelli, M. K.; Ewing, A. G.; Winograd, N., Single-Cell Lipidomics: Characterizing and Imaging Lipids on the Surface of Individual Aplysia californica Neurons with Cluster Secondary Ion Mass Spectrometry. Analytical Chemistry 2013, 85 (4), 2231-2238.

88. Shard, A. G.; Brewer, P. J.; Green, F. M.; Gilmore, I. S., Measurement of sputtering yields and damage in C60 SIMS depth profiling of model organic materials. Surface and Interface Analysis 2007, 39 (4), 294-298.

89. Smith, D. F.; Robinson, E. W.; Tolmachev, A. V.; Heeren, R. M. A.; Paša-Tolić, L., C60 Secondary Ion Fourier Transform Ion Cyclotron Resonance Mass Spectrometry. Analytical Chemistry 2011, 83 (24), 9552-9556.

90. Smith, D. F.; Kiss, A.; Leach, F. E.; Robinson, E. W.; Paša-Tolić, L.; Heeren, R. M. A., High mass accuracy and high mass resolving power FT-ICR secondary ion mass 
spectrometry for biological tissue imaging. Analytical and Bioanalytical Chemistry 2013, 405 (18), 6069-6076.

91. DeBord, J. D.; Smith, D. F.; Anderton, C. R.; Heeren, R. M. A.; Paša-Tolić, L.; Gomer, R. H.; Fernandez-Lima, F. A., Secondary Ion Mass Spectrometry Imaging of $<$ italic >Dictyostelium discoideum</italic> Aggregation Streams. PLoS ONE 2014, 9 (6), e99319.

92. Mahoney, J. F.; Perel, J.; Ruatta, S. A.; Martino, P. A.; Husain, S.; Cook, K.; Lee, T. D., Massive cluster impact mass spectrometry: A new desorption method for the analysis of large biomolecules. Rapid Communications in Mass Spectrometry 1991, 5 (10), 441445.

93. Mahoney, J. F.; Parilis, E. S.; Lee, T. D., Large biomolecule desorption by massive cluster impact. Nuclear Instruments and Methods in Physics Research Section B: Beam Interactions with Materials and Atoms 1994, 88 (1), 154-159.

94. Zhang, J.; Franzreb, K.; Williams, P., Imaging with biomolecular ions generated by massive cluster impact in a time-of-flight secondary ion microscope. Rapid Communications in Mass Spectrometry 2014, 28 (20), 2211-2216.

95. Zhang, J.; Franzreb, K.; Aksyonov, S. A.; Williams, P., Mass Spectra and Yields of Intact Charged Biomolecules Ejected by Massive Cluster Impact for Bioimaging in a Time-of-Flight Secondary Ion Microscope. Analytical Chemistry 2015, 87 (21), 1077910784.

96. Axelsson, J.; Parilis, E. S.; Reimann, C. T.; Sullivan, P.; Sundqvist, B. U. R., Electron emission from conducting surfaces impacted by multiply-charged polyatomic ions. Nuclear Instruments and Methods in Physics Research Section B: Beam Interactions with Materials and Atoms 1995, 101 (4), 343-356.

97. Håkansson, P.; Della-Negra, S.; Mouffron, J. P.; Waast, B.; Sullivan, P. A., Production, acceleration and diagnostics of molecular ions and ionized clusters. Nuclear Instruments and Methods in Physics Research Section B: Beam Interactions with Materials and Atoms 1996, 112 (1-4), 39-47.

98. Sullivan, P. A.; Axelsson, J.; Sundqvist, B. U. R., Light emission from impacts of energetic proteins on surfaces and a light emission detector for mass spectrometry. Rapid Communications in Mass Spectrometry 1995, 9 (5), 377-382.

99. Bouneau, S.; Della-Negra, S.; Depauw, J.; Jacquet, D.; Le Beyec, Y.; Mouffron, J. P.; Novikov, A.; Pautrat, M., Heavy gold cluster beams production and identification. Nuclear Instruments and Methods in Physics Research Section B: Beam Interactions with Materials and Atoms 2004, 225 (4), 579-589. 
100.Fernandez-Lima, F. A.; Post, J.; DeBord, J. D.; Eller, M. J.; Verkhoturov, S. V.; Della-Negra, S.; Woods, A. S.; Schweikert, E. A., Analysis of Native Biological Surfaces Using a 100 kV Massive Gold Cluster Source. Analytical Chemistry 2011, 83 (22), 84488453.

101.Fernandez-Lima, F. A.; Eller, M. J.; DeBord, J. D.; Verkhoturov, S. V.; Della-Negra, S.; Schweikert, E. A., On the surface mapping using individual cluster impacts. Nuclear Instruments and Methods in Physics Research Section B: Beam Interactions with Materials and Atoms 2012, 273, 270-273.

102.Verkhoturov, S. V.; Eller, M. J.; Della-Negra, S.; Rickman, R. D.; Locklear, J. E.; Schweikert, E. A., Statistics of electron and ion emission from single massive cluster impacts. Surface and Interface Analysis 2011, 43 (1-2), 49-52.

103.Fernandez-Lima, F. A.; Eller, M. J.; DeBord, J. D.; Levy, M. J.; Verkhoturov, S. V.; Della-Negra, S.; Schweikert, E. A., Analysis of Fluorescent Proteins with a Nanoparticle Probe. The Journal of Physical Chemistry Letters 2012, 3 (3), 337-341.

104.Heck, C.; Seki, T.; Oosawa, T.; Chikamatsu, M.; Tanigaki, N.; Hiraga, T.; Matsuo, J., ITO surface smoothing with argon cluster ion beam. Nuclear Instruments and Methods in Physics Research Section B: Beam Interactions with Materials and Atoms 2006, 242 (1-2), 140-142.

105.Seki, T.; Murase, T.; Matsuo, J., Cluster size dependence of sputtering yield by cluster ion beam irradiation. Nuclear Instruments and Methods in Physics Research Section B: Beam Interactions with Materials and Atoms 2006, 242 (1-2), 179-181.

106.Nakamura, K.; Houzumi, S.; Toyoda, N.; Mochiji, K.; Mitamura, T.; Yamada, I., Cluster size dependences of bombardment effects using mass-selected gas cluster ion beams. Nuclear Instruments and Methods in Physics Research Section B: Beam Interactions with Materials and Atoms 2007, 261 (1-2), 660-663.

107.Moritani, K.; Hashinokuchi, M.; Nakagawa, J.; Kashiwagi, T.; Toyoda, N.; Mochiji, K., Extremely low-energy projectiles for SIMS using size-selected gas cluster ions. Applied Surface Science 2008, 255 (4), 948-950.

108. Cumpson, P. J.; Portoles, J. F.; Barlow, A. J.; Sano, N., Accurate argon cluster-ion sputter yields: Measured yields and effect of the sputter threshold in practical depthprofiling by x-ray photoelectron spectroscopy and secondary ion mass spectrometry. Journal of Applied Physics 2013, 114 (12), 124313.

109.Bich, C.; Havelund, R.; Moellers, R.; Touboul, D.; Kollmer, F.; Niehuis, E.; Gilmore, I. S.; Brunelle, A., Argon Cluster Ion Source Evaluation on Lipid Standards and Rat Brain Tissue Samples. Analytical Chemistry 2013, 85 (16), 7745-7752. 
110.Delcorte, A.; Restrepo, O. A.; Czerwinski, B., Cluster SIMS of Organic Materials: Theoretical Insights. In Cluster Secondary Ion Mass Spectrometry, John Wiley \& Sons, Inc.: 2013; pp 13-55.

111.Phan, N. T. N.; Fletcher, J. S.; Ewing, A. G., Lipid Structural Effects of Oral Administration of Methylphenidate in Drosophila Brain by Secondary Ion Mass Spectrometry Imaging. Analytical Chemistry 2015, 87 (8), 4063-4071.

112.Tian, H.; Wucher, A.; Winograd, N., Dynamic Reactive Ionization with Cluster Secondary Ion Mass Spectrometry. Journal of The American Society for Mass Spectrometry 2016, 27 (2), 285-292.

113. Angerer, T. B.; Dowlatshahi Pour, M.; Malmberg, P.; Fletcher, J. S., Improved Molecular Imaging in Rodent Brain with Time-of-Flight-Secondary Ion Mass Spectrometry Using Gas Cluster Ion Beams and Reactive Vapor Exposure. Analytical Chemistry 2015, 87 (8), 4305-4313.

114.Hiraoka, K.; Mori, K.; Asakawa, D., Fundamental aspects of electrospray droplet impact/SIMS. Journal of Mass Spectrometry 2006, 41 (7), 894-902.

115. Asakawa, D.; Hiraoka, K., Study of the desorption/ionization mechanism in electrospray droplet impact secondary ion mass spectrometry. Rapid Communications in Mass Spectrometry 2011, 25 (5), 655-660.

116. Asakawa, D.; Hiraoka, K., Detection of peptides in high concentration of salts by electrospray droplet impact/secondary ion mass spectrometry. Surface and Interface Analysis 2011, 43 (10), 1341-1345.

117.Takaishi, R.; Hiraoka, K., Electrospray droplet impact/secondary ion mass spectrometry (EDI/SIMS) using mixed solvents of water/methanol and water/2-propanol as projectile droplets. Surface and Interface Analysis 2014, 46 (6), 418-424.

118. Asakawa, D.; Hiraoka, K., Surface characterization and depth profiling of biological molecules by electrospray droplet impact/SIMS. Surface and Interface Analysis 2012, 44 (2), 227-231.

119.Sheraz née Rabbani, S.; Barber, A.; Fletcher, J. S.; Lockyer, N. P.; Vickerman, J. C., Enhancing Secondary Ion Yields in Time of Flight-Secondary Ion Mass Spectrometry Using Water Cluster Primary Beams. Analytical Chemistry 2013, 85 (12), 5654-5658.

120.Sheraz née Rabbani, S.; Razo, I. B.; Kohn, T.; Lockyer, N. P.; Vickerman, J. C., Enhancing Ion Yields in Time-of-Flight-Secondary Ion Mass Spectrometry: A Comparative Study of Argon and Water Cluster Primary Beams. Analytical Chemistry 2015, 87 (4), 2367-2374. 
121. Yukio, F.; Kouji, W.; Naoaki, S.; Hidehiko, N.; Atsushi, S.; Taisuke, N.; Toshiyuki, F.; Akira, K.; Shingo, I.; Mitsuhiro, T., Ion Beam Generation from an Electrolyte Solution Containing Polyatomic Cations and Anions for Secondary Ion Mass Spectrometry. Japanese Journal of Applied Physics 2009, 48 (12R), 126005.

122.Fujiwara, Y.; Saito, N.; Nonaka, H.; Nakanaga, T.; Ichimura, S., A new cluster-ionbeam source for secondary ion mass spectrometry (SIMS) using the electrospray of a pure ionic liquid under high vacuum. Nuclear Instruments and Methods in Physics Research Section B: Beam Interactions with Materials and Atoms 2010, 268 (11-12), 1938-1941.

123.Fujiwara, Y.; Saito, N.; Nonaka, H.; Nakanaga, T.; Ichimura, S., Characteristics of a charged-droplet beam generated by vacuum electrospray of an ionic liquid. Chemical Physics Letters 2011, 501 (4-6), 335-339.

124.Lozano, P.; Martínez-Sánchez, M., Ionic liquid ion sources: suppression of electrochemical reactions using voltage alternation. Journal of Colloid and Interface Science 2004, 280 (1), 149-154.

125.Chiu, Y. H.; Gaeta, G.; Levandier, D. J.; Dressler, R. A.; Boatz, J. A., Vacuum electrospray ionization study of the ionic liquid, [Emim][Im]. International Journal of Mass Spectrometry 2007, 265 (2-3), 146-158.

126.Perez-Martinez, C.; Guilet, S.; Gierak, J.; Lozano, P., Ionic liquid ion sources as a unique and versatile option in FIB applications. Microelectronic Engineering 2011, 88 (8), 2088-2091.

127.Fujiwara, Y.; Saito, N.; Nonaka, H.; Ichimura, S., Emission characteristics of a charged-droplet beam source using vacuum electrospray of an ionic liquid. Surface and Interface Analysis 2013, 45 (1), 517-521.

128.Fujiwara, Y.; Saito, N., Time-of-flight secondary ion mass spectrometry (TOFSIMS) using an ionic-liquid primary ion beam source. Surface and Interface Analysis 2014, 46 (S1), 348-352.

129.Fujiwara, Y.; Saito, N., Effects of a proton-conducting ionic liquid on secondary ion formation in time-of-flight secondary ion mass spectrometry. Rapid Communications in Mass Spectrometry 2016, 30 (1), 239-249.

130. Adams, A.; Read, F. H., Electrostatic cylinder lenses II: Three element einzel lenses. Journal of Physics E: Scientific Instruments 1972, 5 (2), 150.

131.Moore, J. H.; Davis, C. C.; Coplan, M. A.; Greer, S. C., Building Scientific Apparatus. Cambridge University Press: 2009. 
132.Sise, O.; Ulu, M.; Dogan, M., Multi-element cylindrical electrostatic lens systems for focusing and controlling charged particles. Nuclear Instruments and Methods in Physics Research Section A: Accelerators, Spectrometers, Detectors and Associated Equipment 2005, 554 (1-3), 114-131.

133.Takahiro, S.; Hikaru, K.; Chie, H.; Ken, I.; Iwao, O., Improvement of Focused IonBeam Optics in Single-Ion Implantation for Higher Aiming Precision of One-by-One Doping of Impurity Atoms into Nano-Scale Semiconductor Devices. Japanese Journal of Applied Physics 2002, 41 (3A), L287.

134.Galejs, A.; Kuyatt, C. E., Focusing and dispersing properties of a stigmatic crossedfield energy analyzer. Journal of Vacuum Science \& Technology 1978, 15 (3), 865-867.

135.Calverd, A. M., A simple electron beam switching pulse generator for small accelerators. Nuclear Instruments and Methods 1971, 96 (1), 43-44.

136.(Simion.com), Simion.com,. "SIMION Supplemental Documentation - SIMION 8.1 Supplemental Documentation". N.p., 2016. Web. 1 Mar. 2016. SIMION 8.1 Supplemental Documentation". N.p., 2016. Web. 1 Mar. 2016.

137.Dahl, D. A., simion for the personal computer in reflection. International Journal of Mass Spectrometry 2000, 200 (1-3), 3-25.

138. Cornish, T. J.; Cotter, R. J., A curved-field reflectron for improved energy focusing of product ions in time-of-flight mass spectrometry. Rapid Communications in Mass Spectrometry 1993, 7 (11), 1037-1040.

139. Shaffer, S. A.; Tang, K.; Anderson, G. A.; Prior, D. C.; Udseth, H. R.; Smith, R. D., A novel ion funnel for focusing ions at elevated pressure using electrospray ionization mass spectrometry. Rapid Communications in Mass Spectrometry 1997, 11 (16), 18131817.

140.DeBord, J. D.; Della-Negra, S.; Fernandez-Lima, F. A.; Verkhoturov, S. V.; Schweikert, E. A., Bidirectional Ion Emission from Massive Gold Cluster Impacts on Nanometric Carbon Foils. The Journal of Physical Chemistry C 2012, 116 (14), 81388144.

141.Tempez, A.; Schultz, J. A.; Della-Negra, S.; Depauw, J.; Jacquet, D.; Novikov, A.; Lebeyec, Y.; Pautrat, M.; Caroff, M.; Ugarov, M.; Bensaoula, H.; Gonin, M.; Fuhrer, K.; Woods, A., Orthogonal time-of-flight secondary ion mass spectrometric analysis of peptides using large gold clusters as primary ions. Rapid Communications in Mass Spectrometry 2004, 18 (4), 371-376. 
142.Bich, C.; Touboul, D.; Brunelle, A., Cluster TOF-SIMS imaging as a tool for micrometric histology of lipids in tissue. Mass Spectrometry Reviews 2014, 33 (6), 442451.

143.Carado, A.; Passarelli, M. K.; Kozole, J.; Wingate, J. E.; Winograd, N.; Loboda, A. V., C(60) SIMS with a Hybrid-Quadrupole Orthogonal time-of-flight Mass Spectrometer. Analytical chemistry 2008, 80 (21), 7921-7929.

144.Piehowski, P. D.; Carado, A. J.; Kurczy, M. E.; Ostrowski, S. G.; Heien, M. L.; Winograd, N.; Ewing, A. G., MS/MS Methodology to Improve Sub-Cellular Mapping of Cholesterol Using ToF-SIMS. Analytical chemistry 2008, 80 (22), 8662-8667.

145.Fletcher, J. S.; Rabbani, S.; Henderson, A.; Blenkinsopp, P.; Thompson, S. P.; Lockyer, N. P.; Vickerman, J. C., A New Dynamic in Mass Spectral Imaging of Single Biological Cells. Analytical Chemistry 2008, 80 (23), 9058-9064.

146.Fletcher, J. S.; Vickerman, J. C.; Winograd, N., Label free biochemical 2D and 3D imaging using secondary ion mass spectrometry. Current opinion in chemical biology 2011, 15 (5), 733-740.

147.Fisher, G. L.; Hammond, J. S.; Larson, P. E.; Bryan, S. R.; Heeren, R. M. A., Parallel imaging MS/MS TOF-SIMS instrument. Journal of Vacuum Science \& Technology B 2016, 34 (3), $03 \mathrm{H} 126$. 


\section{Appendices}

\section{APPENDIX A}

\section{LIQUID METAL ION SOURCE FABRICATION}

Read through this entire procedure before attempting to fabricate this source.

\section{Etching Solution Preparation:}

1. Clean all glassware to be used in preparation thoroughly. If the glassware is not clean, the etching solution will be yellow in color (the solution will be less efficient for etching).

2. Prepare an aqueous solution of $35 \% \mathrm{w} / \mathrm{w} \mathrm{NaOH}$ in a plastic bottle. Write the date prepared on the container ( $\mathrm{NaOH}$ should be made fresh every 3 months).

3. Mix in a clean and dry beaker:

$-10 \mathrm{~mL}$ of $35 \% \mathrm{NaOH}$ solution

$-50 \mathrm{~mL}$ of Glycerol

$-50 \mathrm{~mL}$ of distilled water

Stir thoroughly. Prepare the etching solution fresh on the same day as the source preparation. Etching solution rapidly loses etching efficiency as it ages.

\section{Needle Preparation:}

1. Cut a section of tungsten wire $(0.200 \mathrm{~mm}$ dia. $) 5-10 \mathrm{~cm}$ long. Clean withautomotive grade sandpaper (600-grit). This mechanical removal of the oxide layer needs to be done the same day as assembling and dipping the source, so do not prepare needles in bulk and store.

2. Place wire in pin vise and center. Pull the wire with your fingers from the base of the pin vise all the way to the end of the wire in continuous smooth motions. Repeat until the wire is straight. 
3. Cut the wire to a length of $3 \mathrm{~cm}$.

4. Etch the needle using the following procedures:

a. Place the needle pin vise into the Teflon needle brace, and place the needle brace into the Teflon block.

b. Be sure the level of the etching solution is parallel to the base of the platform and perfectly normal to the needle. Use the course adjustment on the platform to bring the solution to the needle. Use the fine adjustment ( $\mathrm{x}-\mathrm{y}$ positioner) on the stand to make the final positional adjustments.

c. The end of the wire can be split or ragged as a result of cutting. This portion must be removed before etching the needle. Attach the electrical connections. Insert about $1 \mathrm{~mm}$ of the needle into the etching solution and turn on the AC voltage to a high setting $(\sim 40 \mathrm{~V})$. Remove the needle from the solution every $\sim 2 \mathrm{~min}$ to check the progress of the etching. Continue until the needle is flat on the bottom. At times, this step can result in a needle being formed-if this happens, skip step 4d.

d. With the voltage off, immerse the needle tip into the etching solution and use the fine adjustment to pull the needle out just enough to maintain the meniscus. Use the fine adjustment on the positioner to ensure that the length of the needle tip submerged is enough to form the proper half-angle. This should be done while looking through the microscope for better accuracy. Apply a low voltage ( $~ 5-10 \mathrm{~V})$ to form the cone at the tip of the needle. Remove the needle from the solution every 
$\sim 5$ min to check the progress of the etching. Continue until the needle cone has a half angle of $\sim 49.5^{\circ}$. The tolerance for cone angle is between $90-100^{\circ}$.

e. After the cone has formed, turn AC voltage off and immerse the needle $15 \mathrm{~mm}$ into the solution. Increase the voltage until very fine bubbles form on the surface of the needle and rise to the surface of the etching solution $\sim 5-10 \mathrm{~V}$. Remove the oxide layer by etching for $\sim 6 \mathrm{~min}$.

f. Rinse the needle with distilled water to remove excess etching solution. Use the needle on the same day as preparation—do not store.

\section{Spring Reservoir Preparation:}

1. Cut a $\sim 20 \mathrm{~cm}$ piece of tungsten wire $(0.200 \mathrm{~mm}$ dia. $)$. Clean with automotive grade sandpaper. Bend wire in various directions with your hand while cleaning to "break" the memory of the wire. Reservoirs should be made on the day of source production so the mechanical removal of oxide layers using sandpaper is not lost.

2. Clean wire form with ethanol to ensure no contamination of the wire. Place center of wire on form and begin to wrap around the form with jerky motions toward previous turn. Be sure to use equal force at top and bottoms of turns.

3. Make 9-10 tight turns to form the spring reservoir (no less than 9). There should be little to no space between the individual turns. If equal force was used in production, a good reservoir should be a barrel, parallel with the base.

4. Cut spring ends to the proper length using the spring jig as a measuring tool Do not bend or crease the legs, they should be straight or just gently curved. 
5. Insert the spring legs into the source assembly and tighten screws gently.

6. Do not over-tighten as that can cause undue stress on the spring - use tweezers to hold the spring legs to keep them from twisting. If the spring is not parallel to the base (i.e. not horizontal) use the spring form to bring it into position.

7. Remove Teflon block from etching stand and attach the trident to split the electrical connections for etching the reservoir.

8. Turn spring assembly $90^{\circ}$ so that it is perpendicular to the outlet of the tube connecting the two glass chambers (and thus the electrical field).

9. Immerse spring into the etching solution and be sure to remove any air bubble caught in center of spring. Attach the electrical connections and apply the same low voltage ( $\sim 5$ $10 \mathrm{~V}$ ) to achieve small, rising bubbles and etch for $5 \mathrm{~min}$. Remove any air bubbles that form in the center of spring during etching. Rinse spring with distilled water to remove excess etching solution.

\section{Inserting Needle into Spring Assembly:}

1. Accurately measure $20 \mathrm{~mm}$ from the tip of the needle and cut off excess.

2. Insert needle into the source assembly and using tweezers position into the middle of the spring reservoir (between spring turn 4 and 5).

3. The reservoir should be centered in the side view of the spring, and must be in a vertical position above the spring at a right angle to the top of the spring. Use millimeter paper to adjust the height of the needle to $1.3 \mathrm{~mm}$ above the top of the spring. In the case that sparking occurs with sources, adjust the needle height above the reservoir to $\sim 1.7$ 
$\mathrm{mm}$ (do not exceed $2 \mathrm{~mm}$ or source will not emit). 1.3-1.5 $\mathrm{mm}$ is ideal for large $\mathrm{Au}$ cluster emission.

4. Tighten the screw of the needle holder gently. Do not cause tension that forces the needle to one side or the other. If this occurs, the needle can move during heating.

5. Dip the entire assembly into etching solution, attach the trident and apply the same low voltage $(\sim 5-10 \mathrm{~V})$ to achieve small, rising bubbles and etch for 5 minutes.

Note: Be sure to remove any air bubbles that form in the center of the reservoir. The time of etching is not critical, the color and finish is what is important. When all of the oxide layer has been removed, the color of the tungsten after rinsing with water should be dark grey and matte (not shiny) in finish.

\section{Inserting Source Assembly into Vacuum:}

1. Attach the source assembly to the vertical translator and attach wires to feedthrough Cover the insulator with a thin Teflon cover to protecting it from tungsten evaporation. Be sure to check connectivity before pumping.

2. Obtain a pellet of the $\mathrm{Au} / \mathrm{Si}$ eutectic (97\% Au / 3\% Si, Academy Precision Metals). Clean it by using a file to remove surface contaminants, use 600-grit followed by 1000grit automotive sandpaper to smooth surface, finally use green abrasive pad to polish. Sonicate in ethanol for 5 min then dry with nitrogen. Place the pellet in the tantalum boat in the vacuum chamber. Fresh pellet dimensions are a cylinder of $3 / 8$ ” height, $\sim 17 \mathrm{~g}$. When the mass of the pellet is $\leq 11 \mathrm{~g}$, another piece of gold should be added. 
3. Clean the chamber and boat with ethanol and place source assembly lid onto vacuum chamber. Evacuate to at least 3x10-6 Torr.

4. Heat the eutectic by ramping the temperature of the tantalum boat (monitored with the thermocouple) at a rate of $10^{\circ} \mathrm{C} / \mathrm{min}$. For the variant power supply, this generally equates to $1 \mathrm{increment} / \mathrm{min}$. The eutectic will melt at a temperature of $\sim 363^{\circ} \mathrm{C}$. This constitutes $\sim 395 \mathrm{mV}$ across the tantalum boat.

5. The color of the eutectic will change from yellow to grey just before melting. Check by gently nudging the chamber-you will see ripples in the eutectic if it has melted; maintain the minimum temperature for complete liquidity. The needle and spring must be free of any contaminants before immersing into the eutectic.

\section{To do this:}

a. Apply $\sim 7-8 \mathrm{~W}$ between the needle and one leg of the spring for $\sim 2 \mathrm{~min} .7-8 \mathrm{~W}$ should produce a red-orange color on the filament, avoid yellow or white-this means the filament is too hot. Check the location of the needle before, during, and after heating to assure the needle does not move when the reservoir springs expand $(\sim 10 \%)$

b. Apply the same power to the needle and the other leg of the spring for $\sim 2 \mathrm{~min}$.

c. Apply $\sim 5 \mathrm{~W}$ of power between the two spring legs (not the needle). The spring will glow a light red color. Lower the needle and spring assembly slowly into the eutectic until the top of the spring is immersed completely. Turn off the power and begin to raise slowly but at a constant rate (do not use jerky motions). 
d. At times the reservoir will not fill the first time, but it should now be "wetted" enough to fill the subsequent times. If the spring is not filled on the first attempt, repeat steps 5a-c, dipping the reservoir completely after each step. This makes a total of 4 dippings. Continue dipping until reservoir is filled.

6. Before breaking vacuum, examine the needle and reservoir with the microscope. A meniscus should be present between the needle and top of the reservoir. The reservoir should be filled with eutectic.

7. Cool the eutectic in the tantalum boat by directly turning the voltage on the boat to zero directly. Wait until the eutectic reaches room temperature before breaking vacuum and removing the source assembly.

8. It is helpful to take a picture of each source and write down its dimensions, and any other parameters of interest into the source notebook, in order to track any abnormalities in emission and lifetime.

\section{Inserting Gold Source Assembly onto Mounting System:}

1. Attach the first extractor and center the needle in the center of the hole using the 4 set screws located at the base. Rotate the extractor and bring the tip of the needle in the plane of the bottom of the extractor cap, using the microscope to view. Tighten in place with the locking washer.

2. Attach brass collar and brass lockring. Attach the second extractor shield and use the microscope to make the final adjustments to the $x-y$ position of the needle. The needle should be centered within the two diaphragms. Tighten the set screws of the extractor. 
3. Install the source onto the assembly flange and connect the high voltage cables to the filament (x2) and the floating voltage cable to the base of the source. Check for proper connectivity before attaching set-up to the instrument. 


\section{APPENDIX B}

\section{ELECTROSPRAY NANOCAPILLARY FABRICATION AND VACUUM ELECTROSPRAY SOURCE DRAWINGS}

\section{Preparing Capillaries for Polyamide Coating Removal.}

1. Uncap NanoStrip (KMG Chemicals) and turn on Hot Plate to 100C (4-5 on settings) to heat up nanostrip solution (30 mins-1 hour).

2. Cleave the desired capillary length utilizing a ceramic cutter (5 inches is generally fine for a vacuum EDI source).

3. Fill the capillary with water utilizing a microsyringe and a syringe pump (ensure that water is flowing out to discard clogged capillaries!).

\section{Stripping the Polyamide Coating from the Capillary.}

4. Set flow rate to $.1 \mu \mathrm{L} / \mathrm{min}$ and check flow afterwards.

5. Check temperature of NanoStrip and if at 100C, dip capillary 1-1.5 cm and leave in heated solution until no polyamide is left on the capillary (emitter looks totally clear). Ensure tips are perpendicular to the solution with no vibration or rattling.

a. Make sure to take note of the time it takes for the NanoStrip to remove the polyamide! Good solutions of NanoStrip should not take longer than 1015 mins to remove polyamide.

b. If the stripping process takes longer than 30 min, neutralize the Nanostrip with $\sim 1 \mathrm{~g}$ of $\mathrm{NaOH}$ before rinsing with water.

6. Rinse tips in water for 5-10 mins to remove residual NanoStrip. 


\section{Etching the Fused Silica to Create a Fine Emitter.}

7. Obtain fresh $49 \%$ HF (Sigma Aldrich) and place in a 50mL PTFE beaker.

a. Make sure its PTFE not glass! HF will etch glassware!

8. Set water flow rate $.1 \mu \mathrm{L} / \mathrm{min}$ to prevent internal etching of the capillary.

9. Immerse the nanocapillary $(20 \mu \mathrm{m} \mathrm{ID} / 159 \mu \mathrm{m}$ OD) for a duration of $30-45$ mins.

a. Make sure to check back periodically as etching times vary depending on the freshness of the HF!

10. Upon effective etching, rinse capillary for 5-10 min.

11. Check under magnifying glass or microscope for any defects to the shape of the tip. 